\title{
A Search for the Standard Model Higgs Boson Produced in Association With TOP QUARKS
}

\author{
DISSERTATION
}

Presented in Partial Fulfillment of the Requirements for the Degree Doctor of Philosophy in the Graduate School of The Ohio State University

$$
\text { By }
$$

Jonathan S. Wilson, B.A., M.S.

Graduate Program in Physics

The Ohio State University

2012

Dissertation Committee:

R. Hughes, Advisor

B. Winer

Y. Kovchegov

T. Lemberger 
(c) Copyright by

Jonathan S. Wilson 


\section{Abstract}

We have performed a search for the Standard Model Higgs boson produced in association with top quarks in the lepton plus jets channel. We impose no constraints on the decay of the Higgs boson. We employ ensembles of neural networks to discriminate events containing a Higgs boson from the dominant $t \bar{t}$ background, and set upper bounds on the Higgs production cross section. At a Higgs boson mass $m_{H}=120 \mathrm{GeV} / c^{2}$, we expect to exclude a cross section 12.7 times the Standard Model prediction, and we observe an exclusion 27.4 times the Standard Model prediction with $95 \%$ confidence. 


\section{VITA}

July $1985 \ldots \ldots \ldots \ldots \ldots \ldots \ldots \ldots \ldots \ldots \ldots \ldots \ldots \ldots \ldots \ldots \ldots$ Born - Richardson, TX

August 2000 - May 2003 .................... V. Berkner High School, Richardson, TX

June 2004 - May 2007 .................. Undergraduate research assistant, Baylor University, Waco, TX

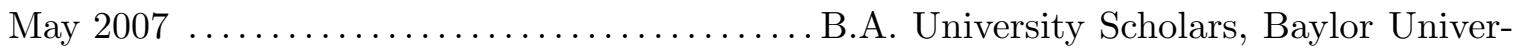
sity, Waco, TX

August 2007 - December 2008 ............. Graduate Teaching Assistant, The Ohio State University, Columbus, $\mathrm{OH}$

January 2009 - December 2011 ............. Graduate Research Assistant, The Ohio State University, Columbus, $\mathrm{OH}$

August 2009 ............................ Physics, The Ohio State University, Columbus, $\mathrm{OH}$

\section{Publications}

"The CDF II eXtremely fast tracker upgrade"

A. Abulencia et al. [CDF Collaboration].

Nucl. Instrum. Meth. A 572, 358 (2007).

"The CDF II 3D-Track Level 2 Trigger Upgrade"

A. Abulencia, P. Azzurri, W. Brian, E. Cochran, J. R. Dittmann, J. Efron, G. Flanagan and R. Forrest et al..

"The CDF II level 1 track trigger upgrade"

A. Abulencia et al. [CDF Collaboration].

IEEE Trans. Nucl. Sci. 55, 126 (2008).

"eXtremely Fast Tracker trigger upgrade at CDF"

A. Abulencia et al. [CDF Collaboration].

Nucl. Instrum. Meth. A 598, 328 (2009). 
"Measurement of the $t \bar{t}$ Production Cross Section with an in situ Calibration of $b$-jet Identification Efficiency"

T. Aaltonen et al. [CDF Collaboration].

arXiv:1007.4423 [hep-ex]

Phys. Rev. D 83, 071102 (2011)

"Diffractive W and Z Production at the Fermilab Tevatron"

T. Aaltonen et al. [CDF Collaboration].

arXiv:1007.5048 [hep-ex]

Phys. Rev. D 82, 112004 (2010)

"Direct Top-Quark Width Measurement CDF"

T. Aaltonen et al. [CDF Collaboration].

arXiv:1008.3891 [hep-ex]

Phys. Rev. Lett. 105, 232003 (2010)

"Measurement of the $W W+W Z$ Production Cross Section Using a Matrix Element Technique in Lepton + Jets Events"

T. Aaltonen et al. [CDF Collaboration].

arXiv:1008.4404 [hep-ex]

Phys. Rev. D 82, 112001 (2010)

"Updated Search for the Flavor-Changing Neutral-Current Decay $D^{0} \rightarrow \mu^{+} \mu^{-}$" T. Aaltonen et al. [CDF Collaboration].

arXiv:1008.5077 [hep-ex]

Phys. Rev. D 82, 091105 (2010)

"Search for the supersymmetric partner of the top quark in ppbar collisions at $\operatorname{sqrt}(\mathrm{s})=1.96 \mathrm{TeV} "$

T. Aaltonen et al. [CDF Collaboration].

arXiv:1009.0266 [hep-ex]

Phys. Rev. D 82, 092001 (2010)

"Improved Search for a Higgs Boson Produced in Association with Z-¿l+l- in proton antiproton Collisions at $\operatorname{sqrt}(\mathrm{s})=1.96 \mathrm{TeV}$ "

T. Aaltonen et al. [CDF Collaboration].

arXiv:1009.3047 [hep-ex]

Phys. Rev. Lett. 105, 251802 (2010)

"Top Quark Mass Measurement in the Lepton + Jets Channel Using a Matrix Element Method and in situ Jet Energy Calibration"

T. Aaltonen et al. [CDF Collaboration].

arXiv:1010.4582 [hep-ex]

Phys. Rev. Lett. 105, 252001 (2010) 
"Search for a New Heavy Gauge Boson $W^{\prime}$ with Electron + missing ET Event Signature in $p \bar{p}$ collisions at $\sqrt{s}=1.96 \mathbf{T e V}$ "

T. Aaltonen et al. [CDF Collaboration].

arXiv:1012.5145 [hep-ex]

Phys. Rev. D 83, 031102 (2011)

"Evidence for a Mass Dependent Forward-Backward Asymmetry in Top Quark Pair Production"

T. Aaltonen et al. [CDF Collaboration].

arXiv:1101.0034 [hep-ex]

Phys. Rev. D 83, 112003 (2011)

"Measurement of the Forward-Backward Asymmetry in the $B \rightarrow K^{(*)} \mu^{+} \mu^{-}$Decay and First Observation of the $B_{s}^{0} \rightarrow \phi \mu^{+} \mu^{-}$Decay"

T. Aaltonen et al. [CDF Collaboration].

arXiv:1101.1028 [hep-ex]

Phys. Rev. Lett. 106, 161801 (2011)

"Production of $\Lambda, L a \bar{m} b d a^{0} \Xi^{ \pm}$and $\Omega^{ \pm}$Hyperons in $p \bar{p}$ Collisions at sqrts $=\mathbf{1 . 9 6}$ TeV"

T. Aaltonen et al. [CDF Collaboration].

arXiv:1101.2996 [hep-ex]

"Search for High Mass Resonances Decaying to Muon Pairs in $\sqrt{s}=1.96$ TeV $p \bar{p}$ Collisions"

T. Aaltonen et al. [The CDF Collaboration].

arXiv:1101.4578 [hep-ex]

Phys. Rev. Lett. 106, 121801 (2011)

"Measurement of the Top Quark Mass in the Lepton+Jets Channel Using the Lepton Transverse Momentum"

T. Aaltonen et al. [The CDF Collaboration].

arXiv:1101.4926 [hep-ex]

Phys. Lett. B 698, 371 (2011)

"Search for heavy bottom-like quarks decaying to an electron or muon and jets in $p \bar{p}$ collisions at $\sqrt{s}=1.96 \mathbf{T e V}^{\prime \prime}$

T. Aaltonen et al. [The CDF Collaboration].

arXiv:1101.5728 [hep-ex]

Phys. Rev. Lett. 106, 141803 (2011)

"Search for the Higgs boson in the all-hadronic final state using the CDF II detector"

T. Aaltonen et al. [The CDF Collaboration].

arXiv:1102.0024 [hep-ex]

Phys. Rev. D 84, 052010 (2011) 
"Observation of the $Y(4140)$ structure in the $J / \psi \phi$ Mass Spectrum in $B^{ \pm} \rightarrow$ $J / \psi \phi K$ decays"

T. Aaltonen et al. [The CDF Collaboration].

arXiv:1101.6058 [hep-ex]

"Observation of $B_{s}^{0} \rightarrow J / \psi K^{* 0}(892)$ and $B_{s}^{0} \rightarrow J / \psi K_{S}^{0}$ Decays"

T. Aaltonen et al. [CDF Collaboration].

arXiv:1102.1961 [hep-ex]

Phys. Rev. D 83, 052012 (2011)

"Search for New Heavy Particles Decaying to $Z Z \rightarrow \ell \ell \ell \ell, \ell \ell j j$ in $p \bar{p}$ Collisions at $\sqrt{s}=1.96$ TeV"

T. Aaltonen et al. [CDF Collaboration].

arXiv:1102.4566 [hep-ex]

Phys. Rev. D 83, 112008 (2011)

"Measurement of the $B_{s}$ Lifetime in Fully and Partially Reconstructed $B_{s} \rightarrow$ $D_{s}^{-}\left(\phi \pi^{-}\right) X$ Decays in $\bar{p}-p$ Collisions at $\sqrt{s}=1.96 \mathbf{T e V}$ "

T. Aaltonen et al. [CDF Collaboration].

arXiv:1103.1864 [hep-ex]

"Search for Production of Heavy Particles Decaying to Top Quarks and Invisible Particles in $p \bar{p}$ collisions at $\sqrt{s}=1.96 \mathbf{T e V}^{\prime}$

T. Aaltonen et al. [CDF Collaboration].

arXiv:1103.2482 [hep-ex]

Phys. Rev. Lett. 106, 191801 (2011)

"Limits on Anomalous Trilinear Gauge Couplings in $Z \gamma$ Events from $p \bar{p}$ Collisions at $\sqrt{s}=1.96 \mathrm{TeV} "$

T. Aaltonen et al. [CDF Collaboration].

arXiv:1103.2990 [hep-ex]

Phys. Rev. Lett. 107, 051802 (2011)

"Measurement of the mass difference between $t$ and $\bar{t}$ quarks"

T. Aaltonen et al. [CDF Collaboration].

arXiv:1103.2782 [hep-ex]

Phys. Rev. Lett. 106, 152001 (2011)

"Measurement of the Top Pair Production Cross Section in the Lepton + Jets Channel Using a Jet Flavor Discriminant"

T. Aaltonen et al. [CDF Collaboration].

arXiv:1103.4821 [hep-ex]

Phys. Rev. D 84, 031101 (2011)

"Search for New Dielectron Resonances and Randall-Sundrum Gravitons at the 
Collider Detector at Fermilab"

T. Aaltonen et al. [CDF Collaboration].

arXiv:1103.4650 [hep-ex]

Phys. Rev. Lett. 107, 051801 (2011)

"Measurement of Event Shapes in Proton-Antiproton Collisions at Center-ofMass Energy 1.96 TeV"

T. Aaltonen et al. [CDF Collaboration].

arXiv:1103.5143 [hep-ex]

Phys. Rev. D 83, 112007 (2011)

"First Measurement of the Angular Coefficients of Drell-Yan $e^{+} e^{-}$pairs in the $\mathrm{Z}$ Mass Region from $p \bar{p}$ Collisions at $\sqrt{s}=1.96 \mathrm{TeV}^{\prime \prime}$

T. Aaltonen et al. [CDF Collaboration].

arXiv:1103.5699 [hep-ex]

Phys. Rev. Lett. 106, 241801 (2011)

"Measurements of Direct CP Violating Asymmetries in Charmless Decays of Strange Bottom Mesons and Bottom Baryons"

T. Aaltonen et al. [CDF Collaboration].

arXiv:1103.5762 [hep-ex]

Phys. Rev. Lett. 106, 181802 (2011)

"Invariant Mass Distribution of Jet Pairs Produced in Association with a $W$ boson in $p \bar{p}$ Collisions at $\sqrt{s}=1.96$ TeV"

T. Aaltonen et al. [CDF Collaboration].

arXiv:1104.0699 [hep-ex]

Phys. Rev. Lett. 106, 171801 (2011)

"Search for the Rare Radiative Decay: $W \rightarrow \pi \gamma$ in $p \bar{p}$ Collisions at $\sqrt{s}=1.96$ TeV"

T. Aaltonen et al. [CDF Collaboration].

arXiv:1104.1585 [hep-ex]

"Search for a Very Light CP-Odd Higgs Boson in Top Quark Decays from $\mathbf{p}^{-} p$ Collisions at $1.96 \mathrm{TeV}^{\prime}$

T. Aaltonen et al. [CDF Collaboration].

arXiv:1104.5701 [hep-ex]

Phys. Rev. Lett. 107, 031801 (2011)

"Top quark mass measurement using the template method at CDF"

T. Aaltonen et al. [CDF Collaboration].

arXiv:1105.0192 [hep-ex]

Phys. Rev. D 83, 111101 (2011) 
"Measurement of the $t \bar{t}$ production cross section in $p \bar{p}$ collisions at $\sqrt{s}=1.96 \mathrm{TeV}$ using events with large Missing $E_{T}$ and jets"

T. Aaltonen et al. [CDF Collaboration].

arXiv:1105.1806 [hep-ex]

Phys. Rev. D 84, 032003 (2011)

"First Search for Multijet Resonances in $\sqrt{s}=1.96$ TeV $p \bar{p}$ Collisions"

T. Aaltonen et al. [CDF Collaboration].

arXiv:1105.2815 [hep-ex]

Phys. Rev. Lett. 107, 042001 (2011)

"Improved determination of the sample composition of dimuon events produced in $p \bar{p}$ collisions at $\sqrt{s}=1.96 \mathbf{T e V}$ "

T. Aaltonen et al. [CDF Collaboration].

arXiv:1105.5787 [hep-ex]

Eur. Phys. J. C 71, 1720 (2011)

"Measurements of the properties of $\Lambda_{c}(2595), \Lambda_{c}(2625), \Sigma_{c}(2455)$, and $\Sigma_{c}(2520)$ baryons"

T. Aaltonen et al. [CDF Collaboration].

arXiv:1105.5995 [hep-ex]

Phys. Rev. D 84, 012003 (2011)

"Measurement of branching ratio and $B_{s}^{0}$ lifetime in the decay $B_{s}^{0} \rightarrow J / \psi f_{0}(980)$ at CDF"

T. Aaltonen et al. [CDF Collaboration].

arXiv:1106.3682 [hep-ex]

Phys. Rev. D 84, 052012 (2011)

"Evidence for $t \bar{t} \gamma$ Production and Measurement of $\sigma_{t} \bar{t} \gamma / \sigma_{t} \bar{t}$ "

T. Aaltonen et al. [CDF Collaboration].

arXiv:1106.3970 [hep-ex]

Phys. Rev. D 84, 031104 (2011)

"Search for Higgs Bosons Produced in Association with b-Quarks"

T. Aaltonen et al. [CDF Collaboration].

arXiv:1106.4782 [hep-ex]

"Measurement of the Cross Section for Prompt Isolated Diphoton Production in $p \bar{p}$ Collisions at $\sqrt{s}=1.96$ TeV"

T. Aaltonen et al. [CDF Collaboration].

arXiv:1106.5131 [hep-ex]

Phys. Rev. D 84, 052006 (2011)

"Measurement of the Cross Section for Prompt Isolated Diphoton Production in $p \bar{p}$ Collisions at $\sqrt{s}=1.96 \mathbf{T e V} "$ 
T. Aaltonen et al. [CDF Collaboration].

arXiv:1106.5123 [hep-ex]

Phys. Rev. Lett. 107, 102003 (2011)

"Study of Substructure of High Transverse Momentum Jets Produced in ProtonAntiproton Collisions at $\sqrt{s}=1.96 \mathrm{TeV}^{\prime}$

T. Aaltonen et al. [CDF Collaboration].

arXiv:1106.5952 [hep-ex]

"Search for $B_{s} \rightarrow \mu^{+} \mu^{-}$and $B_{d} \rightarrow \mu^{+} \mu^{-}$Decays with CDF II"

T. Aaltonen et al. [CDF Collaboration].

arXiv:1107.2304 [hep-ex]

Phys. Rev. Lett. 107, 239903 (2011), [Phys. Rev. Lett. 107, 191801 (2011)]

"Observation of the Baryonic Flavor-Changing Neutral Current Decay $\Lambda_{b} \rightarrow$ $\Lambda \mu^{+} \mu^{-}$

T. Aaltonen et al. [CDF Collaboration].

arXiv:1107.3753 [hep-ex]

Phys. Rev. Lett. 107, 201802 (2011)

"Search for New $T^{\prime}$ Particles in Final States with Large Jet Multiplicities and Missing Transverse Energy in ppbar Collisions at sqrt(s) $=1.96 \mathrm{TeV}^{\prime \prime}$

T. Aaltonen et al. [CDF Collaboration].

arXiv:1107.3574 [hep-ex]

Phys. Rev. Lett. 107, 191803 (2011)

"Observation of the $\Xi_{b}^{0}$ Baryon"

T. Aaltonen et al. [CDF Collaboration].

arXiv:1107.4015 [hep-ex]

Phys. Rev. Lett. 107, 102001 (2011)

"Search for a Heavy Top-Like Quark in $p \bar{p}$ Collisions at $\sqrt{ } s=1.96 \mathbf{T e V}$ "

T. Aaltonen et al. [CDF Collaboration].

arXiv:1107.3875 [hep-ex]

"Measurement of Polarization and Search for CP-Violation in $B_{s}^{0} \rightarrow \phi \phi$ Decays" T. Aaltonen et al. [CDF Collaboration].

arXiv:1107.4999 [hep-ex]

"A Search for resonant production of $t \bar{t}$ pairs in $4.8 \mathrm{fb}^{-1}$ of integrated luminosity of $p \bar{p}$ collisions at $\sqrt{s}=1.96 \mathrm{TeV}$ "

T. Aaltonen et al. [CDF Collaboration].

arXiv:1107.5063 [hep-ex]

Phys. Rev. D 84, 072004 (2011)

"Search for new physics in high $p_{T}$ like-sign dilepton events at CDF II" 
T. Aaltonen et al. [CDF Collaboration].

arXiv:1108.0101 [hep-ex]

Phys. Rev. Lett. 107, 181801 (2011)

"Measurements of the Angular Distributions in the Decays $B \rightarrow K^{(*)} \mu^{+} \mu^{-}$at CDF"

T. Aaltonen et al. [CDF Collaboration].

arXiv:1108.0695 [hep-ex]

"Measurement of the top-quark mass in the lepton+jets channel using a matrix element technique with the CDF II detector"

T. Aaltonen et al. [CDF Collaboration].

arXiv:1108.1601 [hep-ex]

Phys. Rev. D 84, 071105 (2011)

"Search for $W Z+Z Z$ production with MET + jets with $b$ enhancement at $\sqrt{s}=$ 1.96 TeV"

T. Aaltonen et al. [CDF Collaboration].

arXiv:1108.2060 [hep-ex]

"Search for resonant production of $t \bar{t}$ decaying to jets in $p \bar{p}$ collisions at $\sqrt{s}=1.96$ TeV"

T. Aaltonen et al. [CDF Collaboration].

arXiv:1108.4755 [hep-ex]

Phys. Rev. D 84, 072003 (2011)

"Measurements of branching fraction ratios and CP-asymmetries in suppressed $B^{-} \rightarrow D\left(\rightarrow K^{+} \pi^{-}\right) K^{-}$and $B^{-} \rightarrow D\left(\rightarrow K^{+} \pi^{-}\right) \pi^{-}$decays"

T. Aaltonen et al. [CDF Collaboration].

arXiv:1108.5765 [hep-ex]

Phys. Rev. D 84, 091504 (2011)

"Top-quark mass measurement using events with missing transverse energy and jets at CDF"

T. Aaltonen et al. [CDF Collaboration].

arXiv:1109.1490 [hep-ex]

Phys. Rev. Lett. 107, 232002 (2011)

"Search for heavy metastable particles decaying to jet pairs in $p \bar{p}$ collisions at $\sqrt{s}=1.96 \mathbf{T e V}^{\prime \prime}$

T. Aaltonen et al. [CDF Collaboration].

arXiv:1109.3136 [hep-ex]

"Search for a Higgs Boson in the Diphoton Final State in p-pbar Collisions at $\operatorname{sqrt}(\mathrm{s})=1.96 \mathrm{TeV} "$

T. Aaltonen et al. [CDF Collaboration]. 
arXiv:1109.4427 [Unknown]

"Measurement of $\mathbf{C P}$-violating asymmetries in $D^{0} \rightarrow \pi^{+} \pi^{-}$and $D^{0} \rightarrow K^{+} K^{-}$ decays at CDF"

T. Aaltonen et al. [CDF Collaboration].

arXiv:1111.5023 [hep-ex]

\section{Fields of Study}

Major Field: Physics

Studies in experimental high energy physics: Dr. Richard Hughes 


\section{Table of Contents}

Page

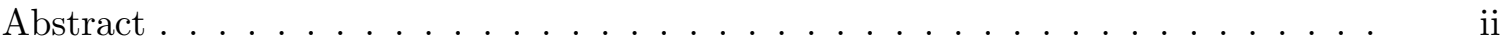

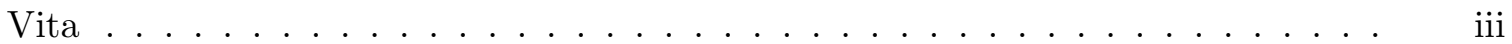

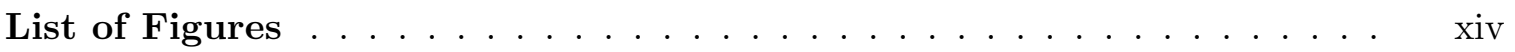

List of Tables . . . . . . . . . . . . . . . . . . . . xvii

\section{Chapters}

1 Theory 1

1.1 Development of the Standard Model . . . . . . . . . . . . . . . . . . 1

1.1.1 The quantum electromagnetic interaction ........... 2

1.1.2 The strong interaction . . . . . . . . . . . . . . . 4

1.1.3 The weak interaction . . . . . . . . . .... 5

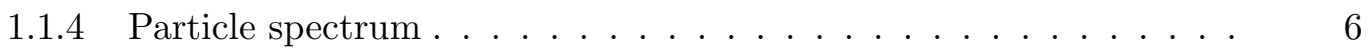

1.2 The Higgs Mechanism . . . . . . . . . . . . . . . . . . . . . . 8

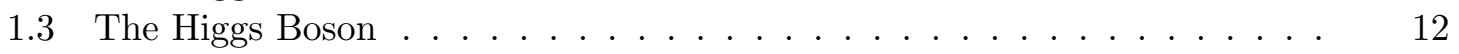

1.3.1 Higgs Decays . . . . . . . . . . . . . . . . . . . . . . . 14

1.3.2 Production Mechanisms . . . . . . . . . . . . . . . . 16

2 The Tevatron and CDF 21

2.1 Accelerator complex . . . . . . . . . . . . . . . . 21

2.2 The Collider Detector at Fermilab . . . . . . . . . . . . . . 24

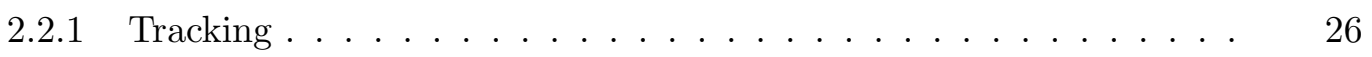

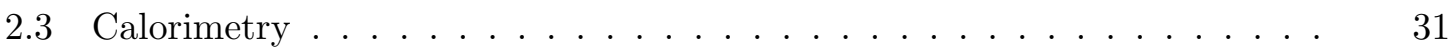

2.4 Muon system . . . . . . . . . . . . . . . . . . . . . . . 32

2.5 Trigger and Data acquisition . . . . . . . . . . . . 33

$\begin{array}{lll}3 & \text { Event Selection } & 37\end{array}$

3.1 Lepton ID . . . . . . . . . . . . . . . . . . . . . . . . . 37

3.2 Missing transverse energy . . . . . . . . . . . . . . . . . 42

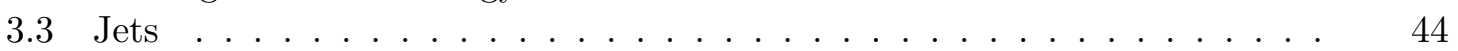

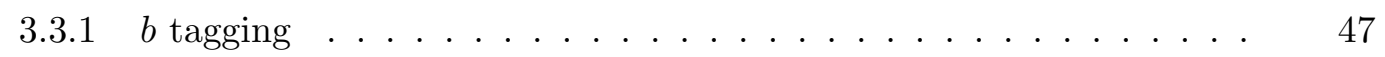

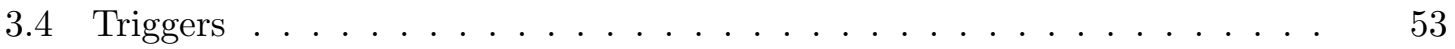

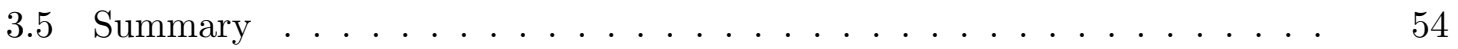

4 Data Modeling $\quad 56$ 
4.1 Monte Carlo simulation . . . . . . . . . . . . . . 56

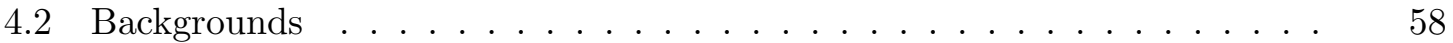

4.2 .1 Irreducible $\ldots \ldots \ldots \ldots \ldots \ldots \ldots \ldots$

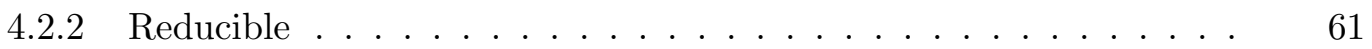

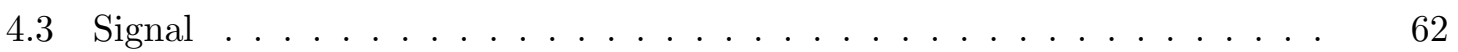

4.4 Estimating the background rates . . . . . . . . . . . . . . 64

4.5 Validating the background model . . . . . . . . . . . . 66

$\begin{array}{lll}5 & \text { Discriminants } & \mathbf{7 1}\end{array}$

5.1 Discriminating variables . . . . . . . . . . . . . . . . 71

5.2 Neural Networks . . . . . . . . . . . . . . . . . . . 76

5.3 Ensemble Method . . . . . . . . . . . . . . . . . 77

5.4 Discriminant outputs . . . . . . . . . . . . . . 78

6 Systematic Uncertainties $\quad 80$

6.1 Initial- and Final-State Radiation . . . . . . . . . . . . . . . . . 80

6.2 Jet Energy Scale . . . . . . . . . . . . . . . . . . . . . 82

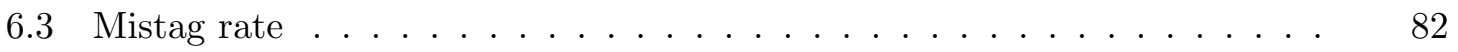

6.4 b-tagging scale factor $\ldots \ldots \ldots \ldots \ldots \ldots$

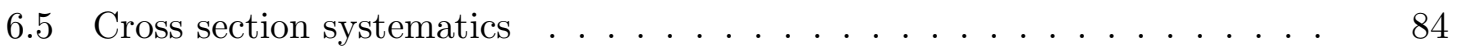

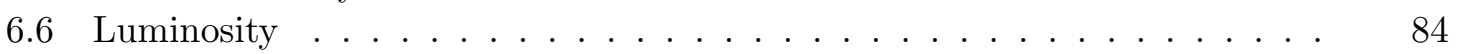

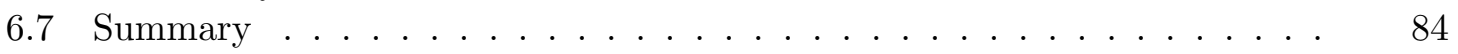

7 Results $\quad \mathbf{8 6}$

7.1 Limit calculation . . . . . . . . . . . . . . . . . . . . . 86

7.1.1 Bayesian limit calculation . . . . . . . . . . . . . . . . 88

7.2 Results. . . . . . . . . . . . . . . . . . 88

8 Conclusion $\quad 91$

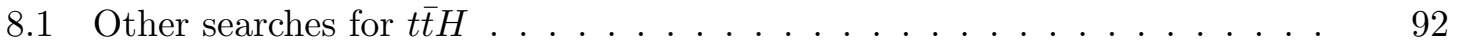

8.2 Combined Higgs searches . . . . . . . . . . . . . . . . . 94

\section{Appendices}

$\begin{array}{ll}\text { A Validation } & 100\end{array}$

A.1 Discriminant inputs . . . . . . . . . . . . . . . . . . . 100

A.1.1 4 jets, $2 b$ tags $\ldots \ldots \ldots \ldots \ldots \ldots \ldots$

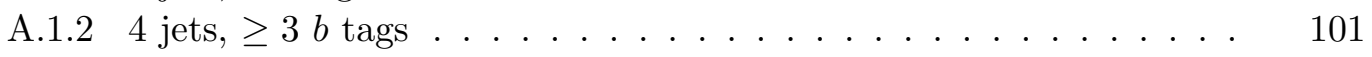

A.1.3 $\geq 5$ jets, $2 b$ tags . . . . . . . . . . . . . . . . . 102

A.1.4 $\geq 5$ jets,$\geq 3 b$ tags $\ldots \ldots \ldots \ldots \ldots \ldots$

A.2 Discriminant outputs . . . . . . . . . . . . . . . . . . 104 


\section{List of Figures}

Figure

Page

1.1 The particle content of the Standard Model of Particle Physics . . . . . . 7

1.2 The potential energy for an Abelian Higgs model . . . . . . . . . . . . . 9

1.3 Fit to global precision electroweak measurements for the mass of the Higgs

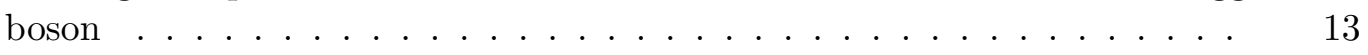

1.4 Decay fractions of the Higgs boson as a function of its mass . . . . . . . . 14

1.5 Cross sections for Higgs production at the Tevatron as a function of Higgs

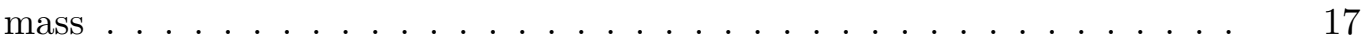

1.6 Higgs boson production via gluon fusion . . . . . . . . . . . . 17

1.7 Higgs boson production via vector boson fusion . . . . . . . . . . . . 18

1.8 Higgs-strahlung with a $W$ or a $Z$ boson . . . . . . . . . . . . . . . . . . . 19

1.9 Top quark associated production with quark and gluon initial states . . . 20

2.1 The Fermilab accelerator complex . . . . . . . . . . . . . . 22

2.2 Tevatron integrated luminosity delivered to and acquired by CDF . . . . 25

2.3 CDF data taking efficiency during Run II . . . . . . . . . . . . 25

2.4 Layout of the components of the CDF tracking system . . . . . . . . . 26

2.5 Electric equipotential contours generated by the field and sense wires in a single COT cell . . . . . . . . . . . . . . . . . . . 27

2.6 End view of the silicon system, showing the Silicon Vertex Detector (SVX) and the Intermediate Silicon Layers (ISL) . . . . . . . . . . . . 28

2.7 Cut-away view of the layout of the Intermediate Silicon Layers (ISL) . . . 29

2.8 Residuals for helix parameters (curvature, impact parameter, $z_{0}$ and $\cot \theta$ ) in

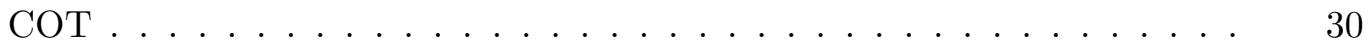

2.9 Helix parameter residuals and transverse momentum resolution of the combined tracking system . . . . . . . . . . . . . . . 31

2.10 Location of tight muons in the detector . . . . . . . . . . . . 32

2.11 Flow of data through the CDF data acquisition and trigger system . . . . 34

2.12 Data flow through the subcomponents of the trigger system . . . . . . . 34

3.1 Production of $t \bar{t} H$, with a charged lepton, a neutrino, four $b$ quarks and two light quarks in the final state . . . . . . . . . . . . . 38

$\begin{array}{lll}3.2 & \text { Distributions and cut locations for central electron identification variables } \quad 39\end{array}$ 
3.3 Distributions and cut locations for plug electron identification variables . 40

3.4 Lepton transverse momentum in $t \bar{t}$ and $t \bar{t} H \ldots \ldots \ldots . . \ldots . . \ldots 41$

3.5 Missing transverse energy distribution in $t \bar{t}$ and $t \bar{t} H \ldots \ldots . \ldots . . \ldots 43$

3.6 Jet energy correction factor as a function of uncorrected transverse energy 44

3.7 Distribution of the number of tight jets in $t \bar{t} H$ signal and $t \bar{t}$ background

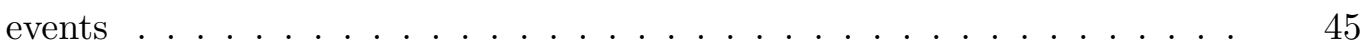

3.8 Distribution of the number of $b$-tagged jets in $t \bar{t} H$ signal and $t \bar{t}$ background

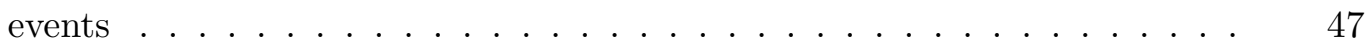

3.9 Positive and negative SECVTX tags . . . . . . . . . . . . 49

3.10 Positive and negative Jet Probability tracks . . . . . . . . . . . . . 50

3.11 Contribution of different Higgs decays to our untagged, 2 tag, and $\geq 3$ tag

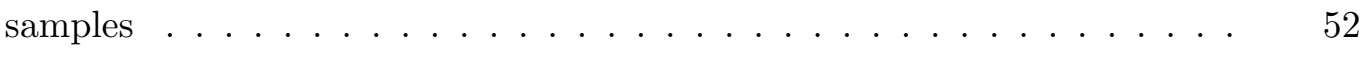

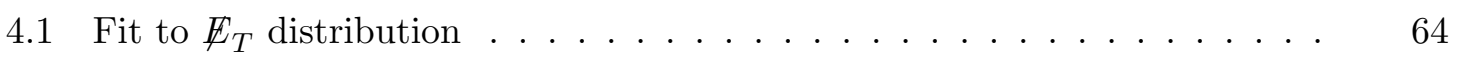

4.2 Leptonic $W$ transverse mass in the 4 jet, 2 tag sample . . . . . . . . . 66

4.3 Hadronic $W$ dijet mass in the 4 jet, 2 tag sample . . . . . . . . . . . 67

4.4 Leptonic $W$ transverse mass in the 4 jet, 3 tag sample . . . . . . . . . . 67

4.5 Leptonic $W$ transverse mass in the 5 jet, 2 tag sample . . . . . . . . . 68

4.6 Leptonic $W$ transverse mass in the 5 jet, 3 tag sample . . . . . . . . . 68

4.7 Hadronic $W$ dijet mass in the 5 jet, 2 tag sample . . . . . . . . . . . . . . 69

4.8 Hadronic $W$ dijet mass in the 5 jet, 3 tag sample . . . . . . . . . . 69

5.1 left: $\Delta R$ between lepton and closest jet (4 jets, 2 tags), right: $\Delta R$ between lepton and $\boldsymbol{E}_{\boldsymbol{T}}(4$ jets, $3+$ tags $) \ldots \ldots \ldots \ldots$. . . . . . . . . 71

5.2 left: $\Delta R$ between lepton and closest tagged jet ( $5+$ jets, 2 tags), right: $\min \Delta R$ between tagged jets $(5+$ jets, $3+$ tags $) \ldots \ldots \ldots . \ldots \ldots$

5.3 left: Lead jet pseudorapidity (4 jets, 2 tags), right: Lead jet transverse energy (4 jets, $3+$ tags $) \ldots \ldots \ldots \ldots \ldots$. . . . . . . . . . . . . . . . . . 72

5.4 left: Second jet $E_{T}(5+$ jets, 2 tags $)$, right: Third jet $E_{T}(5+$ jets, $3+$ tags $) \quad 72$

5.5 left: Lepton $p_{T}(4$ jets, 2 tags $)$, right: $\mathbb{E}_{T}(4$ jets, $3+$ tags $) \ldots \ldots \ldots$

5.6 left: $H_{T}(5+$ jets, 2 tags $)$, right: Mass of all event objects $(5+$ jets, $3+$ tags $) \quad 73$

5.7 left: Minimum dijet mass (4 jets, 2 tags), right: Mass of lepton and jets (4

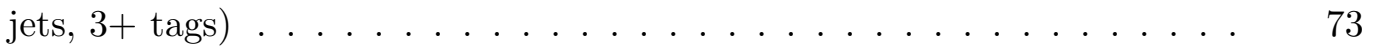

5.8 left: Transverse energy of all event objects ( $5+$ jets, 2 tags), right: $\sum_{\text {jets }} E_{T}$ $(5+$ jets, $3+$ tags $) \ldots \ldots \ldots \ldots \ldots \ldots \ldots \ldots$

5.9 left: Maximum $E_{T}$ of tagged jets (4 jets, 2 tags), right: Mean jet $E_{T}$ (4 jets, $3+$ tags $\ldots \ldots \ldots \ldots \ldots \ldots \ldots$. . . . . . . . . . . . . . . . . 74

5.10 A feed-forward multilayer perception (neural network) architecture . . . . 76

5.11 Discriminant outputs for $m_{H}=115 \mathrm{GeV} / c^{2} \ldots \ldots \ldots \ldots$

6.1 Effect of initial- and final-state radiation on discriminant output for $t \bar{t} H$ signal and $t \bar{t}$ background - effect on $t \bar{t} H$ signal is negligible . . . . . . . . 81

6.2 Effect of JES shifts on discriminant output for $t \bar{t} H$ signal and $t \bar{t}$ background $\quad 81$

6.3 Effect of shifts in the mistag rate on discriminant output for $t \bar{t} H$ signal and $t \bar{t}$ background . . . . . . . . . . . . . . . 83

6.4 Effect of shifts in the $b$-tagging scale factor on discriminant output for $t \bar{t} H$ signal and $t \bar{t}$ background . . . . . . . . . . . . . . 
$7.195 \%$ confidence level limits with 5 or more jets and with 4 jets . . . . .

7.2 Expected and Observed $95 \%$ confidence level limits using all channels com-

bined . . . . . . . . . . . . . . . . . . . . . .

8.1 DØ $2009 t \bar{t} H$ search . . . . . . . . . . . . . . . . . . . . . . 92

8.2 Limits on $t \bar{t} H$ production at $\mathrm{CDF}$ in the zero lepton final state . . . . . 93

8.3 CDF combined limits on Higgs production . . . . . . . . . . . . . . . 94

8.4 Contributions of individual analyses to the CDF combination . . . . . . 95

$8.5 \mathrm{D} \varnothing$ combined limits on Higgs production . . . . . . . . . . . 95

8.6 Tevatron ( $\mathrm{CDF}$ and $\mathrm{D} \varnothing)$ combined limits on Higgs production . . . . . 96 


\section{List of Tables}

Table

Page

3.1 Electron identification cuts . . . . . . . . . . . . . . 38

3.2 Muon identification cuts . . . . . . . . . . . . . . 40

3.3 Number of data events in each analysis channel . . . . . . . . . . . . . . 54

4.1 Background estimates in STSTST category . . . . . . . . . . 58

4.2 Background estimates in STSTJP category . . . . . . . . . . . 59

4.3 Background estimates in STJPJP category . . . . . . . . . . . 59

4.4 Background estimates in STJP category . . . . . . . . . . . 60

4.5 Background estimates in STST category . . . . . . . . . . . . 60

4.6 Predicted $t \bar{t} H$ production cross section $[1,2] \ldots \ldots \ldots \ldots$

4.7 Expected number of selected $t \bar{t} H$ events with at least two $b$ tags in $7.5 \mathrm{fb}^{-1} \quad 63$

6.1 Effects of the systematic uncertainties on the $t \bar{t} H$ signal $\left(m_{H}=115 \mathrm{GeV} / c^{2}\right)$ and $t \bar{t}$ background for the 4-jet channels. Uncertainties are relative, in percent, and are symmetric unless otherwise indicated. . . . . . . . . . . . .

6.2 Effects of the systematic uncertainties on the $t \bar{t} H$ signal $\left(m_{H}=115 \mathrm{GeV} / c^{2}\right)$ and $t \bar{t}$ background for the 5 -jet channels. Uncertainties are relative, in percent, and are symmetric unless otherwise indicated. . . . . . . . . . .

7.1 Expected and Observed $95 \%$ confidence level limits using all channels combined . . . . . . . . . . . . . . . . . 


\section{Chapter 1 THEORY}

The Standard Model of particle physics is a very successful theory that accurately predicts nearly all phenomena that have been observed in particle physics[3]. It unifies the strong, weak, and electromagnetic interactions under an $\mathrm{SU}(3) \times \mathrm{SU}(2) \times \mathrm{U}(1)$ gauge symmetry. The history of the Standard Model is a history of the idea of symmetry as a driving force for physical theories.

\subsection{Development of the Standard Model}

James Clerk Maxwell, in 1865, wrote down 20 differential equations that successfully described all known electric and magnetic phenomena[4]. These equations dealt with the electric scalar potential and the magnetic vector potential, and their solutions were not unique. The potentials could be modified by any scalar field and still give the same physical result, making Maxwell's electrodynamics the first instance of theory with a local, or gauge, symmetry.

In 1887, Albert Michelson and Edward Morley carried out an experiment designed to measure the flow of the luminiferous aether past the Earth[5]. By measuring the interference of a split coherent light beam with itself after traveling in two perpendicular directions, they found that there was no measurable directional variation of the speed of light, as predicted by the aether theory. This experiment indicated that the theory of the luminiferous aether was incorrect, and, along with Maxwell's electrodynamics, directly led to Albert Einstein's

work on special relativity[6]. Special relativity, in essence, asserts that the Poincaré group is 
a fundamental global symmetry group of the universe. This work laid the foundation for quantum field theory and the Standard Model by establishing both the primary importance of symmetry principles and the Poincaré group (with its subgroup the Lorentz group) as the first fundamental global symmetry.

Following the success of both quantum mechanics and relativity, there were many efforts to produce a unified theory that predicted the results of both relativity and quantum mechanics. The crucial task in this endeavor was to develop a version of Schrödinger's equation that respected the Lorentz symmetry. This was accomplished in two ways: the second order differential Klein-Gordon equation, which describes spin-zero particles like the pion, and the first order Dirac equation, which describes spin-1/2 particles like the electron. In particular, it is important to note that, in order to produce a first-order equation that respected the Lorentz symmetry of special relativity, Dirac found it necessary for the wave function to have four components, which he correctly identified as the components of Pauli's spin theory[7]. This is among the great triumphs of symmetry as a predictive principle.

\subsubsection{The quantum electromagnetic interaction}

Once a relativistic quantum mechanical description of the electron was developed, it was natural to attempt to include the interaction of the electron with the electromagnetic field. This is achievable via "minimal coupling",

$$
\left(-i \gamma^{\mu}\left(\partial_{\mu}-i e A_{\mu}\right)+m\right) \psi=0
$$

which includes only the charge $e$ and electron field $\psi$ coupled to the electromagnetic 4potential $A_{\mu}$ and not any higher multipole moments. It is important to note that this theory respects a symmetry

$$
\psi \rightarrow e^{i \theta} \psi
$$

which has the structure of the U(1) group. Minimal coupling in the Dirac equation successfully reproduces classical electrodynamics in the low-field and low-energy limit, but outside of that limit, predicts some additional effects such as negative energy solutions, non-conservation 
of probability, and Zitterbewegung. These effects were viewed as problematic or even paradoxical[8].

The solution to the problems of relativistic quantum mechanics was to quantize the fields themselves. The problems then became reinterpreted as antimatter (negative energy states), particle pair production (non-conservation of probability), and interference between the matter and antimatter states (Zitterbewegung). In this formalism, the minimal coupling to, and even the existence of the electromagnetic 4-potential $A_{\mu}$ can be motivated by changing the $\mathrm{U}(1)$ global symmetry to a local symmetry

$$
\psi \rightarrow e^{i \theta(x)} \psi
$$

which leads to the Lagrangian density (pre-minimal coupling)

$$
\begin{aligned}
\mathcal{L} & =\bar{\psi}(i \not \supset-m) \psi \\
& \rightarrow \bar{\psi} e^{-i \theta(x)}(i \not \supset-m) e^{i \theta(x)} \psi \\
& =\bar{\psi} e^{-i \theta(x)}(i \not \supset-\not \partial \theta(x)-m) \psi .
\end{aligned}
$$

The local $\mathrm{U}(1)$ symmetry is not respected, unless we change $\partial_{\mu}$ to a covariant derivative

$$
D_{\mu} \equiv \partial_{\mu}-i e \partial_{\mu} \theta(x)
$$

Then we can very naturally identify $\partial_{\mu} \theta(x)$ as $A_{\mu}$, the electromagnetic 4-potential. Under the $\mathrm{U}(1)$ symmetry, the 4-potential then transforms as

$$
A_{\mu} \rightarrow A_{\mu}-\frac{1}{e} \partial_{\mu} \theta(x),
$$

and we can write a Lagrangian which includes the kinetic term for the field and respects the $\mathrm{U}(1)$ local symmetry,

$$
\mathcal{L}=\bar{\psi}(i \not \partial-m) \psi-\frac{1}{4}\left(\partial_{\mu} A_{\nu}-\partial_{\nu} A_{\mu}\right)^{2}-e \bar{\psi} \gamma^{\mu} \psi A_{\mu}
$$

This theory was the first example of the predictive power of a local or gauge symmetry. Once Feynman, Dyson, and Tomanaga established methods for performing calculations in 
this theory using renormalization, quantum electrodynamics (QED) quickly became the most precisely tested scientific theory in history.

\subsubsection{The strong interaction}

Many phenomena were not explained in QED, such as nuclear binding, the proliferation of hadrons, and nuclear beta decay. In 1932, James Chadwick discovered the neutron, which was observed to have almost precisely the same mass, spin, gyromagnetic ratio, and nuclear binding properties as the proton. Werner Heisenberg proposed a new SU(2) symmetry analogous to quantum spin that related the proton to the neutron, called isospin. Since this symmetry is not exact, we should expect to find massive pseudo-Goldstone scalar bosons. Work by Hideki Yukawa suggested that interaction with a massive scalar could be responsible for the nuclear binding force, and further estimated that, given the size of the nucleus, the mass of such a particle should be about $100 \mathrm{MeV} / c^{2}$. In 1947, Cecil Powell et al discovered the charged pion in cosmic rays using photographic emulsions on a mountaintop.

In subsequent years, an enormous number of similar particles were discovered, and in 1962, Murray Gell-Mann (and Kazuhiko Nishijima independently) developed the quark model to classify them. The quark model classifies the hadrons according to two quantum numbers, isospin and strangeness. These quantum numbers are dictated by the number of various flavors of quarks, then-hypothetical particles that make up the hadrons. In the original quark model, three quark flavors existed (called up, down, and strange), and these were related by an approximate $\mathrm{SU}(3)$ symmetry.

The discovery of the $\Omega^{-}$baryon, which consists of three strange quarks with identical spins, suggested that quarks might possess an additional quantum number, since such a state would be forbidden by Pauli's exclusion principal otherwise. This realization led to the introduction of an $\mathrm{SU}(3)$ gauge symmetry (different from the $\mathrm{SU}(3)$ flavor symmetry) called quantum chromodynamics, or QCD, to describe the force that bound the quarks together into hadrons via the exchange of gluons. QCD is known experimentally to exhibit a phenomenon called confinement; quarks and gluons cannot be isolated and are only observed as components of hadrons. In 1973, Gross, Wilczek and Politzer discovered that 
the $\mathrm{SU}(3)$ gauge theory possessed a property called asymptotic freedom, which allowed perturbation theory to be used to calculate predictions using this theory. In 1979, the PETRA electron-positron collider produced three-jet events consistent with the predictions of $\mathrm{QCD}[9]$.

\subsubsection{The weak interaction}

Nuclear beta decay is a phenomenon which drove two significant advances in particle physics, the prediction of neutrinos and the development of theories of the weak interaction. Lise Meitner and Otto Hahn's 1911 experiments with beta decay demonstrated that the outgoing electron had a continuous kinetic energy spectrum, rather than the single spike expected due to the conservation of energy and momentum[10]. This lead Pauli to propose the existence of a light, undetected particle, which Fermi called a "neutrino"[11]. Fermi developed a field theoretic description of beta decay as a four-fermion contact interaction, but this theory was not renormalizable, and so only first order calculations could be performed[12].

One candidate theory to remedy this problem was developed by Glashow, Weinberg, and Salam[13, 14, 15]. This theory unifies the electromagnetic and weak interactions under the gauge symmetry $\mathrm{SU}(2) \times \mathrm{U}(1)$. This theory predicts four gauge bosons: three from $\mathrm{SU}(2)$ $\left(W^{+, 0,-}\right)$ and one from $\mathrm{U}(1)(B)$, which mix to form the photon field

$$
A_{\mu}=\cos \theta_{W} B_{\mu}+\sin \theta_{W} W_{\mu}^{0}
$$

and the $Z$ boson field

$$
Z_{\mu}=-\sin \theta_{W} B_{\mu}+\cos \theta_{W} W_{\mu}^{0}
$$

where $\theta_{W}$ is the weak mixing angle. The $W^{+}, W^{-}$, and $Z$ bosons are massive with

$$
m_{W}=m_{Z} \cos \theta_{W}
$$

and the Fermi four-fermion contact interaction emerges as an effective field theory at low energies by integrating out the $W$ degrees of freedom. All fields possess quantum numbers related to these new gauge symmetries. The U(1) quantum number is called weak hypercharge 
$Y_{W}$, and the $\mathrm{SU}(2)$ quantum number is weak isospin $T$. After mixing, the electric charge is

$$
Q=T_{3}+\frac{Y_{W}}{2}
$$

This theory, in addition to predicting two new massive vector bosons, predicts a new weak interaction between particles via the exchange of a $Z$ boson. This neutral current interaction occurs even between particles that have zero electric charge, unlike the electromagnetic interaction, but at low energies, the interaction between charged particles is dominated by the electromagnetic interaction. Neutrinos do not couple to the photon, so the Gargamelle collaboration in 1973 used a beam of neutrinos to observe weak neutral current interactions between energetic neutrinos and nuclei in a bubble chamber. This observation strongly suggested that the electroweak theory was the correct solution.

Although observation of weak neutral currents lent strong support to the electroweak theory, the predicted massive particles had still not been directly observed. In the early 1980s, the Super Proton-Antiproton Synchrotron at CERN delivered colliding proton and antiproton beams to the UA1 and UA2 experiments at a center of mass energy of $540 \mathrm{GeV}$. This was a sufficiently high energy to produce the $W$ and $Z$ bosons, and the discovery of these particles by UA1 and UA2 was announced in 1983[16, 17]. This discovery cemented the electroweak theory's primacy and was another triumph for symmetry principles.

\subsubsection{Particle spectrum}

The Standard Model contains 3 generations of fermions and four gauge bosons, the gluon, the $W$, the $Z$, and the photon, all shown in figure 1.1. The photon is the carrier of the electromagnetic interaction, the gluon carries the strong interaction, and the massive $W$ and $Z$ are responsible for the weak interaction. The fermions appear in two varieties, quarks and leptons. The quarks experience the strong, weak, and electromagnetic interactions. The leptons experience the weak and electromagnetic interactions, but do not couple to the gluon and so do not experience the strong interaction. Each generation of quarks and leptons contains two particles which are related by the (broken) weak isospin symmetry. The up-type quarks $u, c$, and $t$, and the neutrinos $\nu_{e}, \nu_{\mu}$, and $\nu_{\tau}$ have isospin $T_{3}=+1 / 2$, 


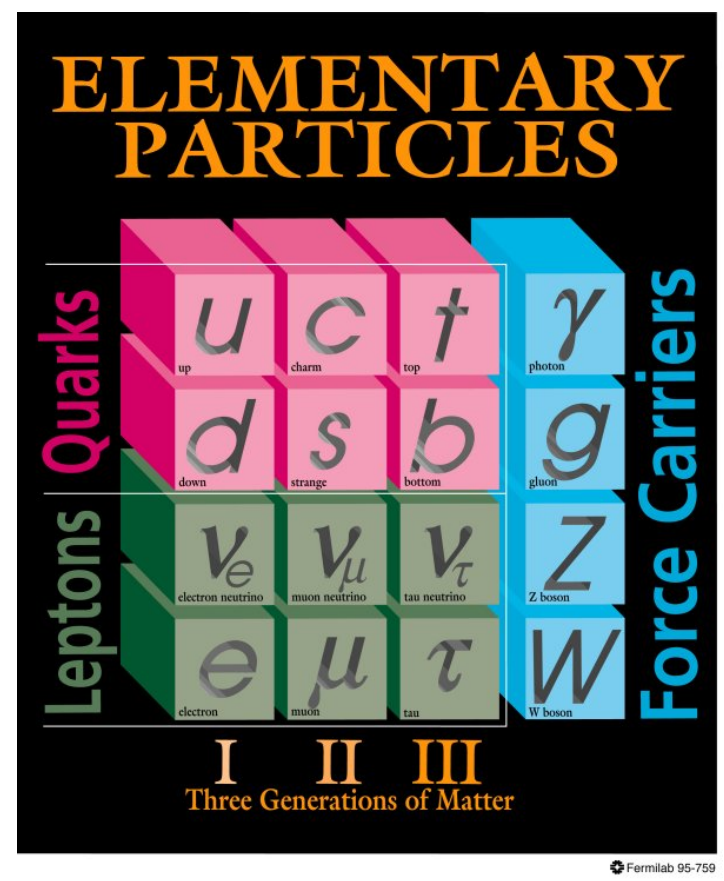

Figure 1.1: The particle content of the Standard Model of Particle Physics

while the down-type quarks $d, s$, and $b$, and the charged leptons $e, \mu$, and $\tau$ have isospin $T_{3}=-1 / 2$.

The up and down quarks and the electron compose all normal matter, since all of their heavier counterparts are unstable. The strange quark was discovered as a component of the kaons in 1947. Prior to the discovery of the kaons, charge conjugation times parity $(C P)$ was expected to be a valid symmetry of the universe, but kaon decay was observed to violate $C P$ in 1964. Kobayashi and Maskawa explained $C P$ violation in kaons by extending the Glashow-Iliopoulos-Maiani mechanism and Cabibbo's $d-s$ mixing angle to a $3 \times 3$ matrix mixing the down and strange quarks with an unobserved third down-type quark, the bottom quark. This required the existence not only of the bottom quark, but also two unobserved up-type quarks, charm and top. In 1974, the $J / \psi$ meson, which contains the charm quark, was discovered independently at the Stanford Linear Accelerator Center (SLAC) by the SPEAR experiment and by Samuel Ting using the Alternating Gradient Synchrotron at Brookhaven. This was quickly followed by the discovery of the bottom quark bearing $\Upsilon$ 
meson at Fermilab in 1977. The extremely massive top quark was observed by CDF and DØ at the Tevatron in 1995[18], completing the discovery of the particles necessitated by Kobayashi and Maskawa's model. Searches for fourth-generation quarks have set strong lower bounds on the mass of any additional quarks.

The electron has been known since the 1896 experiments of J.J. Thomson with cathode rays and is the lightest of the charged leptons, as well as the only stable charged lepton. The electron neutrino was postulated by Pauli to explain the spectrum of electron momenta from nuclear beta decay and later observed using nuclear beta capture by Cowan et al[19]. The other lepton generations are motivated by the need to cancel anomalies in the electroweak theory, which requires that the sum of the weak hypercharge over all fermions (counting the quarks three times apiece for each color) is zero. This motivation was, however, not yet appreciated at the time of the discovery of the muon by Anderson and Neddermeyer in cosmic rays at Caltech in 1936. The confirmation of the muon observation prompted I.I. Rabi to famously exclaim "Who ordered that?" as the existence of a heavy copy of the electron was completely unexpected. Following the observation of the muon and the electron neutrino, it was reasonable to suppose the existence of a second neutrino associated with the muon as well. This muon neutrino was observed by Lederman, Schwartz, and Steinberger in 1962. In 1975, Martin Perl discovered yet another charged lepton, dubbed the $\tau$. The existence of a third lepton generation, complete with the tau neutrino discovered in 2000 by the DONUT collaboration, suggested that there might be an infinite sequence of heavier leptons (the sequential lepton model). However, no additional leptons have been observed, and quite stringent lower bounds have been set on the mass of a hypothetical fourth generation lepton or neutrino[3].

\subsection{The Higgs Mechanism}

The $\mathrm{SU}(3) \times \mathrm{SU}(2) \times \mathrm{U}(1)$ gauge symmetry principles govern the Standard Model, but the mass terms for the massive $W$ and $Z$ gauge bosons do not respect the gauge symmetries. However, due to the finite range of the weak interaction, as well as direct observation, 


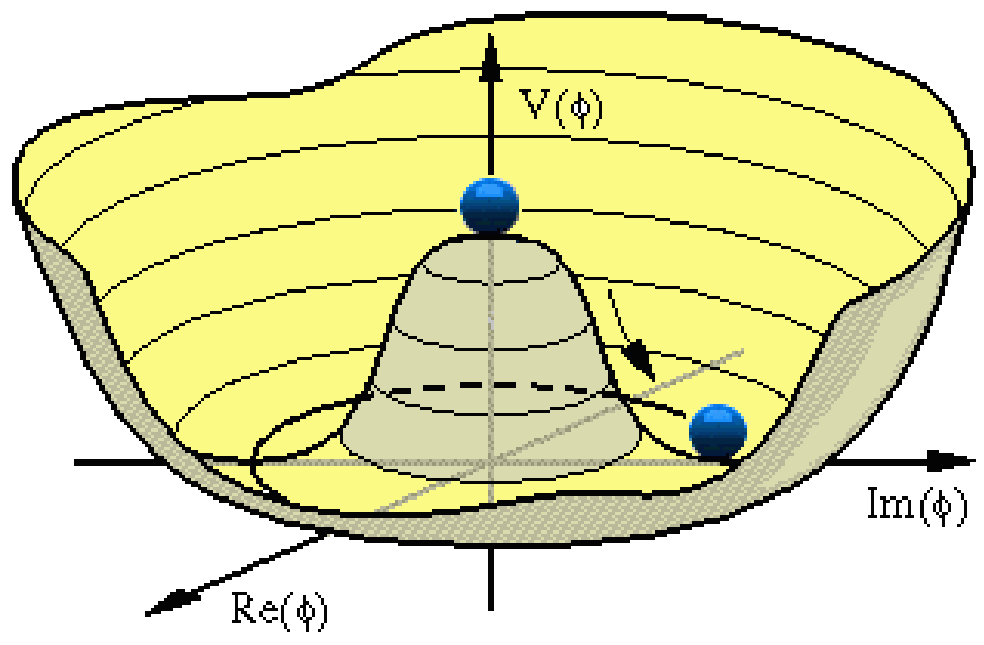

Figure 1.2: The potential energy for an Abelian Higgs model

we know that the $W$ and $Z$ bosons are massive. The gauge symmetry must be broken spontaneously to produce the gauge boson masses.

In the early 1960s, three independent papers were published by Brout and Englert[20], Higgs[21], and Guralnik, Hagen, and Kibble[22] which solved this problem quite handily. The inclusion of an additional field, an $\mathrm{SU}(2)$ doublet and Lorentz scalar, with positive quartic and negative quadratic potential terms, produces a vacuum state which does not respect the $\mathrm{SU}(2) \times \mathrm{U}(1)$ symmetry and is degenerate. The vacuum expectation value of this field produces the weak vector boson masses without the fundamental Lagrangian violating the gauge symmetry. This theory, as noted by Higgs, also predicts an additional neutral scalar boson, which has become known as the Higgs Boson.

With the Higgs Mechanism, the electroweak theory can be made renormalizable and the gauge symmetry respected in every term of the Lagrangian. The electroweak Lagrangian (we neglect the strong interaction for the purposes of discussion) before symmetry breaking is

$$
\mathcal{L}=\mathcal{L}_{\text {gauge }}+\mathcal{L}_{\text {fermions }}+\mathcal{L}_{\text {Higgs }}+\mathcal{L}_{\text {Yukawa }}
$$

where $\mathcal{L}_{\text {gauge }}$ contains the kinetic terms and self interaction among the $\mathrm{SU}(2) \times \mathrm{U}(1)$ gauge fields (the three $A$ fields from $\mathrm{SU}(2)$ with their field strength tensor $F$ and the $B$ field from 
$\mathrm{U}(1)$ with its field strength tensor $G)$,

$$
\mathcal{L}_{\text {gauge }}=-\frac{1}{4} F_{\mu \nu}^{i} F^{i \mu \nu}-\frac{1}{4} G_{\mu \nu} G^{\mu \nu}
$$

$\mathcal{L}_{\text {fermions }}$ contains the kinetic terms for the fermion fields $\psi$ including the gauge covariant derivatives $D_{\mu}$,

$$
\mathcal{L}_{\text {fermions }}=\bar{\psi} i \gamma^{\mu} D_{\mu} \psi
$$

$\mathcal{L}_{\text {Higgs }}$ contains the kinetic and self interaction terms for the Higgs field $\Phi$ including the Higgs potential $V(\Phi)$,

$$
\mathcal{L}_{\text {Higgs }}=\left(D_{\mu} \Phi\right)^{\dagger}\left(D^{\mu} \Phi\right)-V(\Phi)
$$

and $\mathcal{L}_{\text {Yukawa }}$ contains the Yukawa couplings between the Higgs field and the fermion fields,

$$
\mathcal{L}_{\text {Yukawa }}=f^{(e)} \bar{l}_{L} \Phi l_{R}+f^{(u)} \bar{q}_{L} \tilde{\Phi} u_{R}+f^{(d)} \bar{q}_{L} \Phi d_{R}+\text { h.c. }
$$

where $\tilde{\Phi}=i \tau_{2} \Phi^{*}$, and $l, u, d$, and $q$ are the lepton, up-type quark, down-type quark, and quark fields, respectively. The Higgs potential contains a quadratic term with a negative coefficient and a quartic term with a positive coefficient,

$$
V(\Phi)=-\mu^{2} \Phi^{\dagger} \Phi+\lambda\left(\Phi^{\dagger} \Phi\right)^{2}
$$

leading to a potential like the one shown for an Abelian Higgs model in figure 1.2. This potential has a degenerate local minimum for a non-zero field. The minimum energy states are the vacuum states, so the Higgs field has a non-zero vacuum expectation value. The vacuum states are not invariant under the $\mathrm{SU}(2) \times \mathrm{U}(1)$ symmetry group action, so the gauge symmetry, while respected by the Lagrangian, is broken by the vacuum.

After transforming to one of the vacuum states, we can separated our Higgs field $\Phi$ into the vacuum expectation value $v$ and fluctuations $\eta$ and $\boldsymbol{\zeta}$ around the VEV, where $\eta$ describes the fluctuations toward and away from the zero-field state, and the three-component $\boldsymbol{\zeta}$ field describes the fluctuations from one degenerate vacuum state to another.

$$
\Phi^{\prime}=U(\zeta) \Phi=e^{i \zeta \cdot \tau / v} \Phi=\left(\begin{array}{c}
0 \\
\frac{v+\eta}{\sqrt{2}}
\end{array}\right)
$$


where $\boldsymbol{\tau}$ are the Pauli matrices. In order to remain self-consistent, we must also transform the left handed fermion fields and the gauge fields,

$$
\psi_{L}^{\prime}=U(\zeta) \psi_{L}, \quad B_{\mu}^{\prime}=B_{\mu}, \quad \frac{\boldsymbol{\tau} \cdot \boldsymbol{A}_{\mu}^{\prime}}{2}=U(\zeta)\left(\frac{\boldsymbol{\tau} \cdot \boldsymbol{A}_{\mu}^{\prime}}{2}\right) U^{-1}(\zeta)
$$

The transformed SU(2) gauge fields now involve the $\boldsymbol{\zeta}$ degrees of freedom of the Higgs field.

After this transformation, we can rewrite the terms of the Lagrangian. The covariant derivative of the Higgs field becomes

$$
D_{\mu} \Phi^{\prime}=\left(\partial_{\mu}-i \frac{g}{2} \boldsymbol{\tau} \cdot \boldsymbol{A}_{\mu}^{\prime}-i \frac{g^{\prime}}{2} B_{\mu}^{\prime}\right)\left(\begin{array}{c}
0 \\
\frac{v+\eta}{\sqrt{2}}
\end{array}\right),
$$

so then the kinetic term in $\mathcal{L}_{\text {Higgs }}$ includes the terms

$$
\frac{v^{2}}{8}\left\{g^{2}\left[\left(A_{\mu}^{\prime 1}\right)^{2}+\left(A_{\mu}^{\prime 2}\right)^{2}\right]+\left(g A_{\mu}^{\prime 3}-g^{\prime} B_{\mu}^{\prime}\right)^{2}\right\}
$$

This now contains quadratic terms in the gauge fields, which correspond to dynamically generated gauge boson masses. Rewriting these as the more familiar $W$ and $Z$ fields, we have

$$
M_{W}^{2} W_{\mu}^{+} W^{-\mu}+\frac{1}{2} M_{Z}^{2} Z_{\mu} Z^{\mu}
$$

where $W^{ \pm}=A^{\prime 1} \mp i A^{\prime 2} / \sqrt{2}$ and $Z=\cos \theta_{W} A^{\prime 3}-\sin \theta_{W} B^{\prime}$. The $\boldsymbol{\zeta}$ degrees of freedom of the Higgs field have become absorbed or "eaten" by the gauge boson fields, providing them with the additional degree of freedom necessary to have a longitudinal polarization, which only massive particles exhibit. The remaining portions of the $A^{\prime 3}$ and $B^{\prime}$ fields become the photon field

$$
\gamma=\sin \theta_{W} A^{\prime 3}+\cos \theta_{W} B^{\prime}
$$

which remains massless.

In addition to generating the gauge boson masses, symmetry breaking has some other effects. The neutral component of the $\mathrm{SU}(2)$ gauge fields and the $\mathrm{U}(1)$ field mix as described above to become the $Z$ boson and the photon fields. The transformed Lagrangian and the vacuum states respect the residual U(1) symmetry generated by the photon field, which 
produces the electromagnetic interaction. The remaining degree of freedom of the Higgs field, $\eta$, is not absorbed by any other field, and retains a quadratic, cubic, and quartic potential term, corresponding to mass and self-interactions,

$$
V\left(\Phi^{\prime}\right)=\mu^{2} \eta^{2}+\lambda v \eta^{3}+\frac{\lambda}{4} \eta^{4}
$$

This field is the real, scalar Higgs boson field, with $m_{\eta}=m_{H}=\mu \sqrt{2}$. The Yukawa interaction terms become

$$
\begin{aligned}
\mathcal{L}_{\text {Yukawa }}= & \frac{\eta}{\sqrt{2}}\left[f^{(e)} \bar{e}_{L}^{\prime} e_{R}^{\prime}+f^{(u)} \bar{u}_{L}^{\prime} u_{R}^{\prime}+f^{(d)} \bar{d}_{L}^{\prime} d_{R}^{\prime}\right] \\
& +\frac{v}{\sqrt{2}}\left[f^{(e)} \bar{e}_{L}^{\prime} e_{R}^{\prime}+f^{(u)} \bar{u}_{L}^{\prime} u_{R}^{\prime}+f^{(d)} \bar{d}_{L}^{\prime} d_{R}^{\prime}\right]+\text { h.c. },
\end{aligned}
$$

showing the Yukawa interaction of the fermions with the Higgs boson, and the dynamically generated fermion mass terms

$$
m_{e}=f^{(e)} v / \sqrt{2}, \quad m_{u}=f^{(u)} v / \sqrt{2}, \quad m_{d}=f^{(d)} v / \sqrt{2},
$$

where the mass of each fermion is determined by the strength of the Yukawa coupling for that fermion[12].

The Higgs mechanism or something like it is necessary in order to make the electroweak theory renormalizable and well-defined, and it predicts the observed phenomenology of heavy weak vector bosons, photon and $Z$ mixing, and fermion masses. It additionally predicts a heavy, as yet unobserved scalar particle called the Higgs boson.

\subsection{The Higgs Boson}

Everything about the Standard Model Higgs boson is known except for its mass and whether or not it exists. The Higgs mass is not predicted by the Standard Model, but it is related via second- and higher-order effects to many of the parameters of the theory, such as the $W$ and $Z$ boson masses, the top quark mass, and the strong coupling constant. Precise measurement of these parameters can establish bounds on the mass of the Higgs boson in the Standard Model. The GFitter collaboration has performed a meta-analysis of these precise 


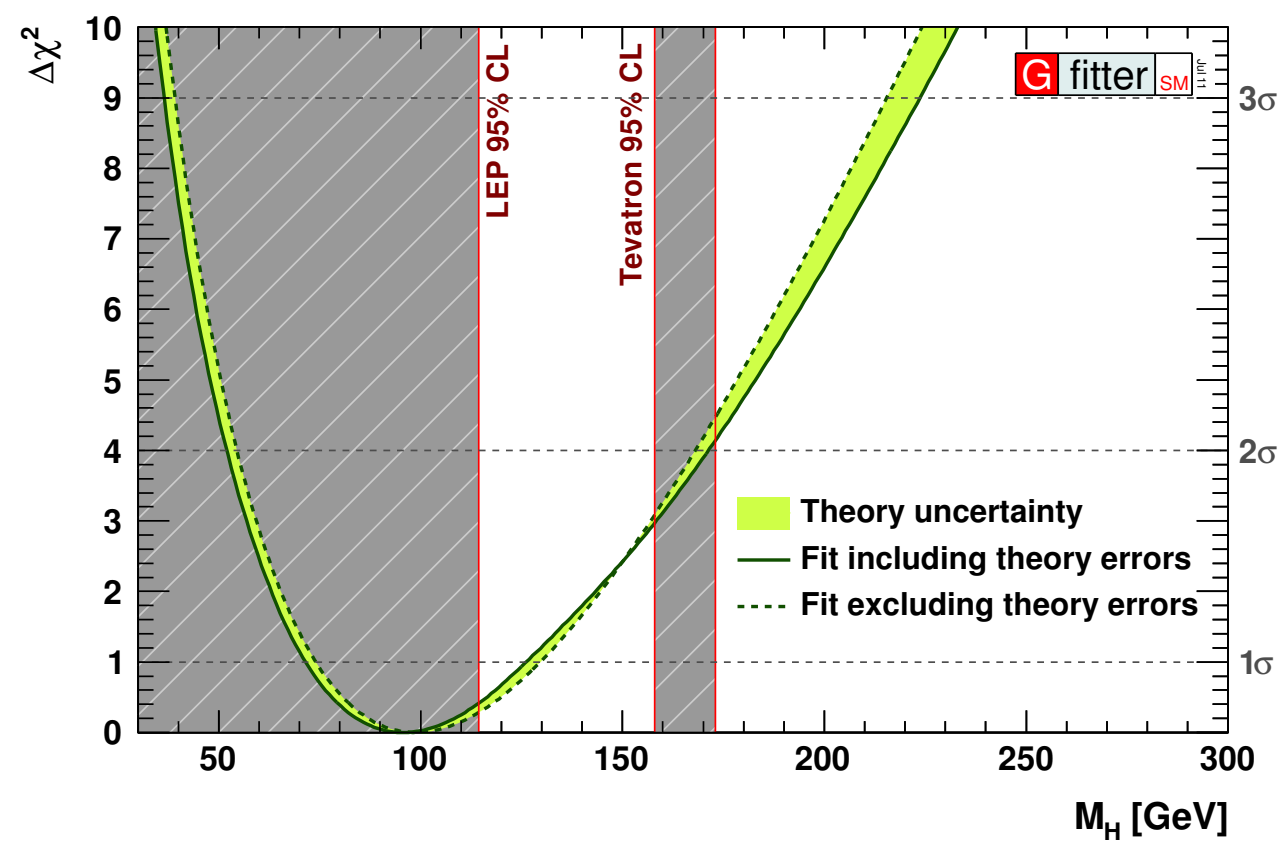

Figure 1.3: Fit to global precision electroweak measurements for the mass of the Higgs boson

measurements, and finds that the most preferred value for the Higgs boson mass is $95 \mathrm{GeV} / c^{2}$, with $95 \%$ confidence level bounds on the Higgs mass of $52<m_{H}<169 \mathrm{GeV} / c^{2}$, as shown in figure 1.3. These meta-analysis results are summarized by figure 1.3[23]. Additionally, some values of the Higgs boson mass pose theoretical difficulties. If the Higgs mass is much larger than $1 \mathrm{TeV} / c^{2}$, then the probability of some events occurring is greater than 1 , which is impossible. If the Higgs mass is too small (less than about $130 \mathrm{GeV} / c^{2}$ ), then the interactions between the top quark and the Higgs field render the Standard Model vacuum unstable, requiring additional new physics to protect the vacuum.

Direct searches for the Higgs boson have been performed at various colliders. The most stringent limits prior to the Tevatron and LHC searches were set by the Large Electron Positron collider (LEP), which found that the Higgs mass was no smaller than $114.4 \mathrm{GeV} / c^{2}$ with $95 \%$ confidence[24]. Since the searches at LEP, the Tevatron and the LHC have also performed searches, and have excluded a Higgs boson with a mass inside the ranges $156<m_{H}<177 \mathrm{GeV} / c^{2}$ and $100<m_{H}<108 \mathrm{GeV} / c^{2}$ (Tevatron), or outside the range 


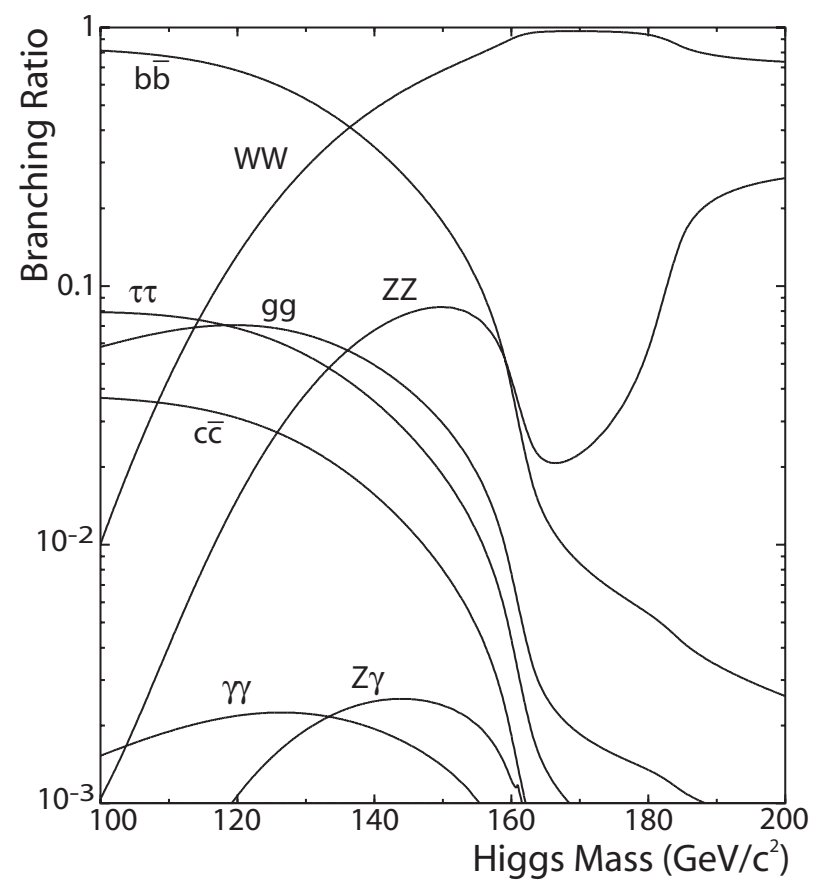

Figure 1.4: Decay fractions of the Higgs boson as a function of its mass

$115<m_{H}<127 \mathrm{GeV} / c^{2}(\mathrm{LHC})$.

As with any predicted new physics, to search for the Higgs boson, the ways in which it can be produced and decay must be considered. The rate of Higgs production relative to the background rates in all the possible final states determines which analyses are likely to yield significant sensitivity and be worth pursuing. At a hadron collider, QCD multijet production is an extremely large background and is difficult to model, so any final states consisting only of jets (from quarks, gluons, and hadronically decaying $\tau$ leptons) is very difficult to search for. Final states containing some combination of electrons, muons, and missing transverse energy have drastically reduced QCD backgrounds compared to the size of the signal and other backgrounds, making these final states the preferred search channels.

\subsubsection{Higgs Decays}

The decay of the Higgs boson is heavily dependent on its mass (figure 1.4). At high mass, the decay $H \rightarrow W^{+} W^{-}$is dominant, and at low mass, $H \rightarrow b \bar{b}$ is dominant, but many of 
the secondary decays are very important as well.

\section{$\boldsymbol{H} \rightarrow \boldsymbol{W}^{+} \boldsymbol{W}^{-}$}

If the Higgs mass is above $\sim 135 \mathrm{GeV} / c^{2}$, then the largest branching ratio is $H \rightarrow W^{+} W^{-}$. Each $W$ boson then decays, $10 \%$ of the time each to $e \nu_{e}, \mu \nu_{\mu}$, and $\tau \nu_{\tau}$, and $\sim 33 \%$ of the time each to $c \bar{s}$ and $u \bar{d}$. The variety of $W$ boson decays lends significant complexity to searches for this mode. As long as at least one of the $W$ bosons decays leptonically (to an electron or muon, plus a neutrino), the backgrounds are small and mostly well understood. In the case where both $W$ bosons decay hadronically (to a quark pair or to a hadronically decaying $\tau$ ), QCD multijet production becomes a significant background. Since QCD multijet production is extremely large and is difficult to model, the all-hadronic decay mode is best exploited in an associated production channel.

\section{$H \rightarrow b \bar{b}$}

Conversely, if the Higgs mass is below $\sim 135 \mathrm{GeV} / c^{2}$, then the largest branching ratio is

$H \rightarrow b \bar{b}$. In this case, QCD multijet production is also a significant background when the Higgs is produced exclusively. Therefore this dominant low-mass decay is also best exploited in an associated production channel.

$H \rightarrow \gamma \gamma / Z \gamma$

The diphoton decay of the Higgs produces a very clean signature with extremely good mass resolution, but the branching ratio is very small. The low rate of $H \rightarrow \gamma \gamma$ makes it very difficult to exploit without a much larger dataset than is available from the Tevatron.

\section{$H \rightarrow Z Z$}

Similar to $H \rightarrow W^{+} W^{-}, H \rightarrow Z Z$ is principally a high-mass decay, and also has considerable complexity due to the variety of decays of the two $Z$ bosons.

Like $H \rightarrow \gamma \gamma$, the four-lepton $H \rightarrow Z Z$ final state provides a clean signal with very good mass resolution, but at a low rate. Other final states with larger backgrounds and poorer 
mass resolution are two leptons and two jets, two leptons and missing transverse energy from two neutrinos, two jets and missing transverse energy, and all jets. In the last two, the QCD multijet background again becomes a major concern.

$H \rightarrow \tau \tau$

The Higgs decay to a $\tau$ pair is a very important decay for supersymmetric Higgs models, some of which predict strong couplings between the Higgs and the third generation fermions. For a Standard Model Higgs, $H \rightarrow \tau \tau$ is still an important secondary search channel. The $\tau$ is non-trivial to reconstruct in the detector as it decays via emission of a $W$ boson before being detected. The decay always involves the emission of a $\nu_{\tau}$, so events with a $\tau$ are never fully reconstructed. The $W$ boson may then go to an electron or muon (and accompanying neutrino) or to a quark pair, so the $\tau$ is detected as either a charged lepton or as a narrow jet. Except in the case where both $\tau$ leptons decay hadronically, the presence of an electron or muon holds down the QCD multijet background to a manageable level.

\section{Other decays}

The Higgs can also decay to a pair of any massive fermions or a pair of gluons. These decays are all either too difficult to separate from large backgrounds or the branching ratios are too small to make them significant. They enter into some analyses that use inclusive signal models, but are otherwise unexploited.

\subsubsection{Production Mechanisms}

There are 4 main ways in which the Higgs can be produced at a hadron collider: gluon fusion, vector boson fusion, vector boson associated production, and top quark associated production. These all have different cross sections with different mass dependences (figure 1.5), and they have different final states and significant backgrounds. 


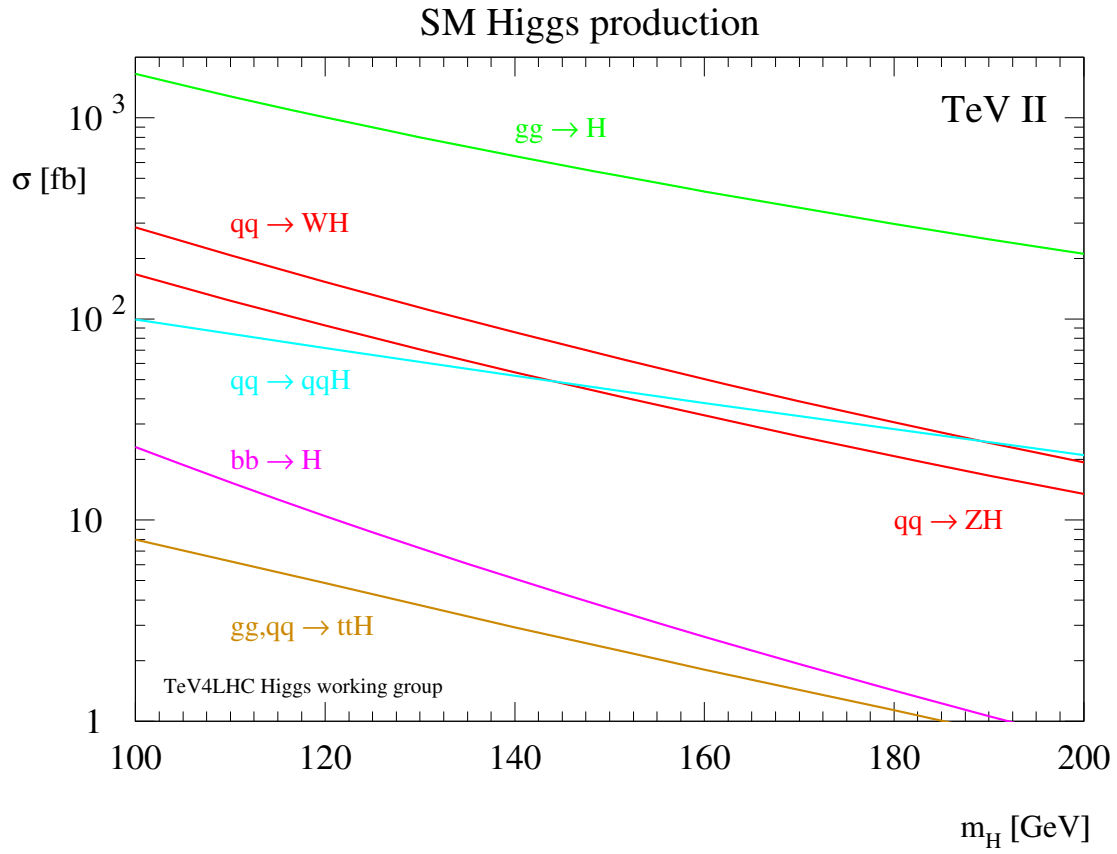

Figure 1.5: Cross sections for Higgs production at the Tevatron as a function of Higgs mass

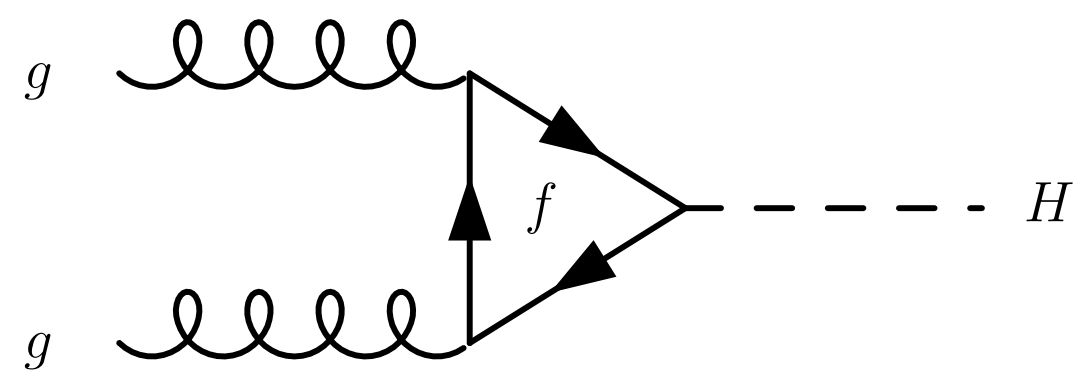

Figure 1.6: Higgs boson production via gluon fusion 


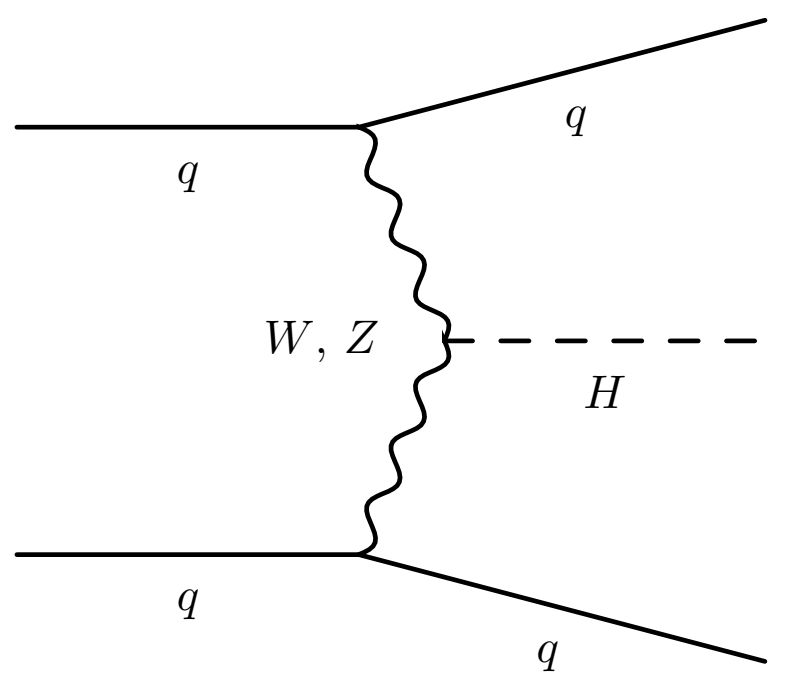

Figure 1.7: Higgs boson production via vector boson fusion

\section{Gluon fusion}

Gluon fusion has by far the largest production cross section of any Higgs production process. Two gluons in the initial state produce the Higgs via a quark loop. Because of the large top-Higgs coupling, the top quark is the largest contributor to the loop (figure 1.6).

While gluon fusion has a large cross section, no other high transverse momentum final state particles are produced. In the low mass region, where the Higgs principally decays

to $b \bar{b}$, this is a very difficult process to search for due to the enormous $b \bar{b}$ background from QCD. In analyses that exploit decays other than $b \bar{b}$, such as $H \rightarrow \gamma \gamma$ or $H \rightarrow W W$, gluon fusion is the largest contributor to the signal acceptance.

\section{Vector boson fusion}

Vector boson fusion has a cross section comparable to vector boson associated production (" $q q \rightarrow q q h "$ in figure 1.5), but much smaller than gluon fusion. Two quarks in the initial state exchange a weak vector boson $W$ or $Z$, which both have large couplings to the Higgs, and may radiate a Higgs boson (figure 1.7).

This process does have additional final state particles in addition to the Higgs decay products. The two quarks continue with only a small momentum exchange, and may be 


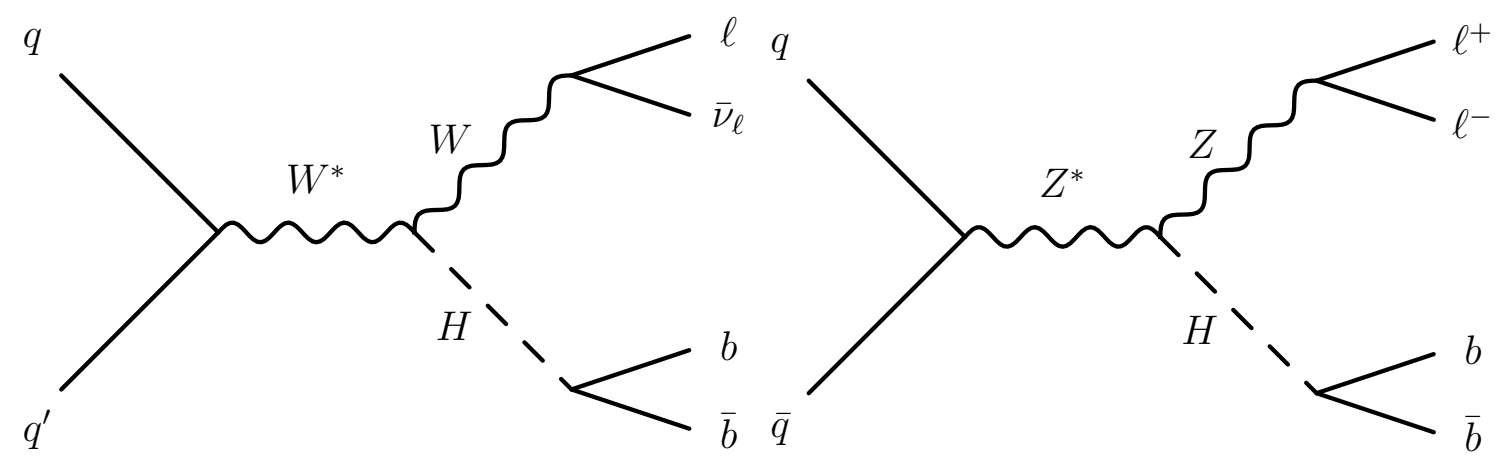

Figure 1.8: Higgs-strahlung with a $W$ or a $Z$ boson

found as jets in the far forward regions of the detector. When the Higgs decays to photons or $W$ bosons, this process contributes a small amount to the signal acceptance. For $H \rightarrow b \bar{b}$, the presence of the two additional forward jets helps to distinguish Higgs events from the QCD background, but this process is principally exploited in $H \rightarrow \tau \tau$ searches, often for a supersymmetric Higgs boson.

\section{Vector boson associated production}

Vector boson associated production, also called "Higgs-strahlung", occurs when a quarkantiquark pair from the final state annihilate to an off-shell $W$ or a $Z$ boson, which then gives up its excess energy by radiating a Higgs boson (figure 1.8). This process has a comparable cross section to the vector boson fusion process, but has a much more distinctive final state as a result of the on-shell $W$ or $Z$ in the final state.

\section{Top quark associated production}

Top quark associated production has the lowest cross section of all Higgs production processes, but, because the top quark has such a large coupling to the Higgs, when a top quark pair is produced, the chance that a Higgs will be radiated is significant (figure 1.9. Top quark associated production also has the most distinctive final state of any Higgs production channel. Each top quark decays to a $W$ boson and $b$ quark, so we can identify $t \bar{t} H$ events by searching for a lepton and missing energy from the leptonic decay of one $W$ boson, two 


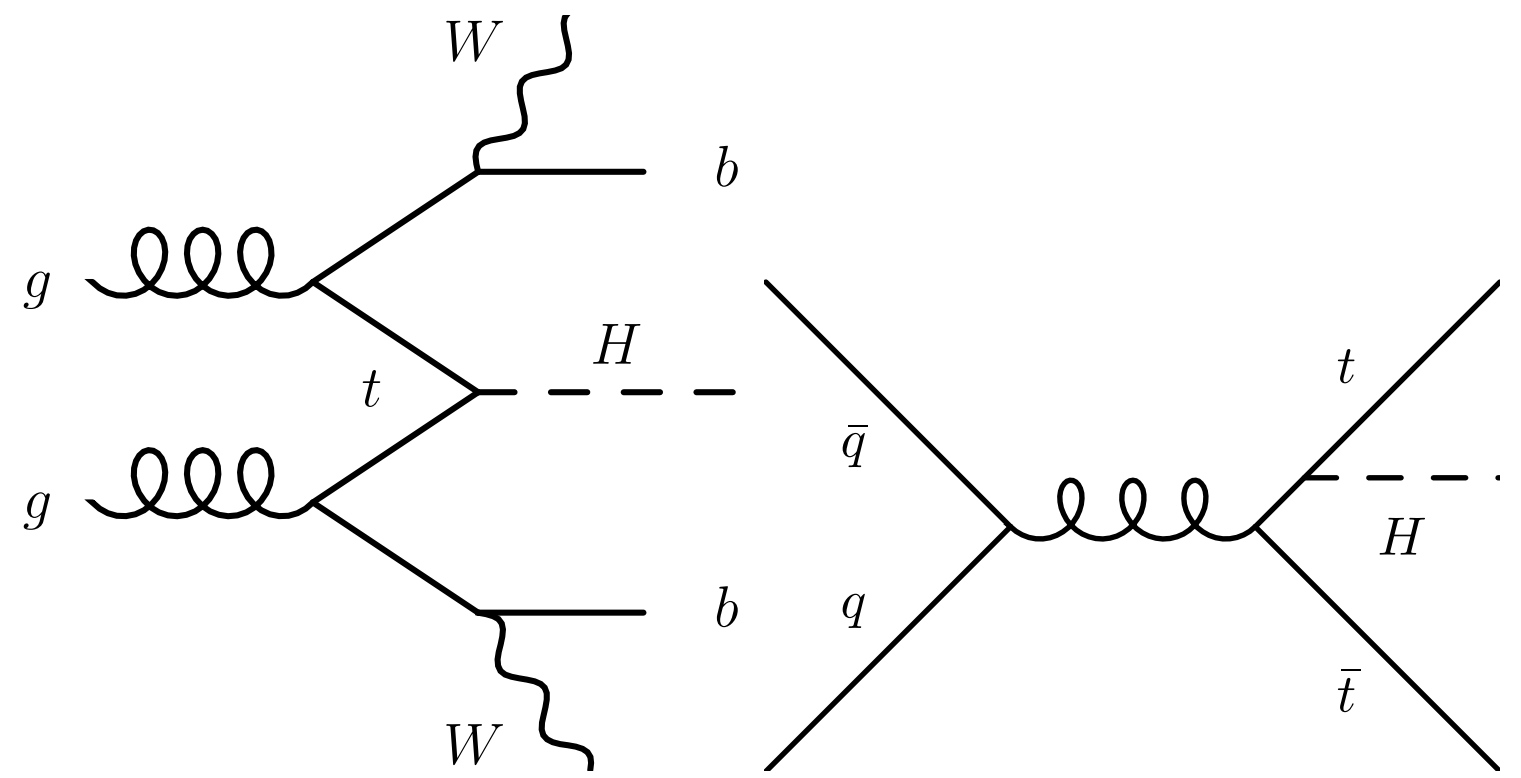

Figure 1.9: Top quark associated production with quark and gluon initial states

$b$-tagged jets, two untagged jets with the mass of the $W$ boson, and additional particles from the decay of the Higgs. The large number of uncommon final state objects from the top decay allows us to select events without requiring any particular Higgs decay final state, which significantly increases the acceptance, particularly in the $135 \mathrm{GeV} / c^{2}$ region where the $H \rightarrow b \bar{b}$ and $H \rightarrow W^{+} W^{-}$branching ratios cross. 


\section{Chapter 2 \\ The Tevatron And CDF}

The Tevatron is a $1 \mathrm{~km}$ radius synchrotron which collides proton and anti-proton beams with a center of mass energy of $1.96 \mathrm{TeV}$. It is located at the Fermi National Accelerator Laboratory in Batavia, Illinois in the central United States. Colliding beams from October 1985 through September 2011, the Tevatron is the final accelerator in the Fermilab accelerator complex and delivers collisions to the $\mathrm{CDF}$ and $\mathrm{D} \varnothing$ detectors at two points around the accelerator ring.

\subsection{Accelerator complex}

Fermilab boasts a large chain of accelerators (figure 2.1) which accelerate hydrogen ions, produce and store anti-protons, send beams of neutrinos through the earth, provide neutron beams for cancer therapy, and provide beams of protons, mesons, and neutrons to various fixed-target experiments in addition to colliding proton and anti-proton beams. The starting point for the accelerators is a bottle of compressed hydrogen gas, from which small puffs of gas are drawn every $66 \mathrm{~ms}$. The atoms in the gas are ionized by the addition of an electron and accelerated to $750 \mathrm{keV}$ by the Cockcroft-Walton preaccelerator, which is a large cascade voltage multiplier producing a static potential of $750000 \mathrm{~V}$. The accelerated ions are then transferred to the Linear Accelerator.[25]

Linac The Linear Accelerator, or Linac, is composed of a series of superconducting radio frequency cavities separated by drift tubes. It produces an $H^{-}$beam at an energy of 


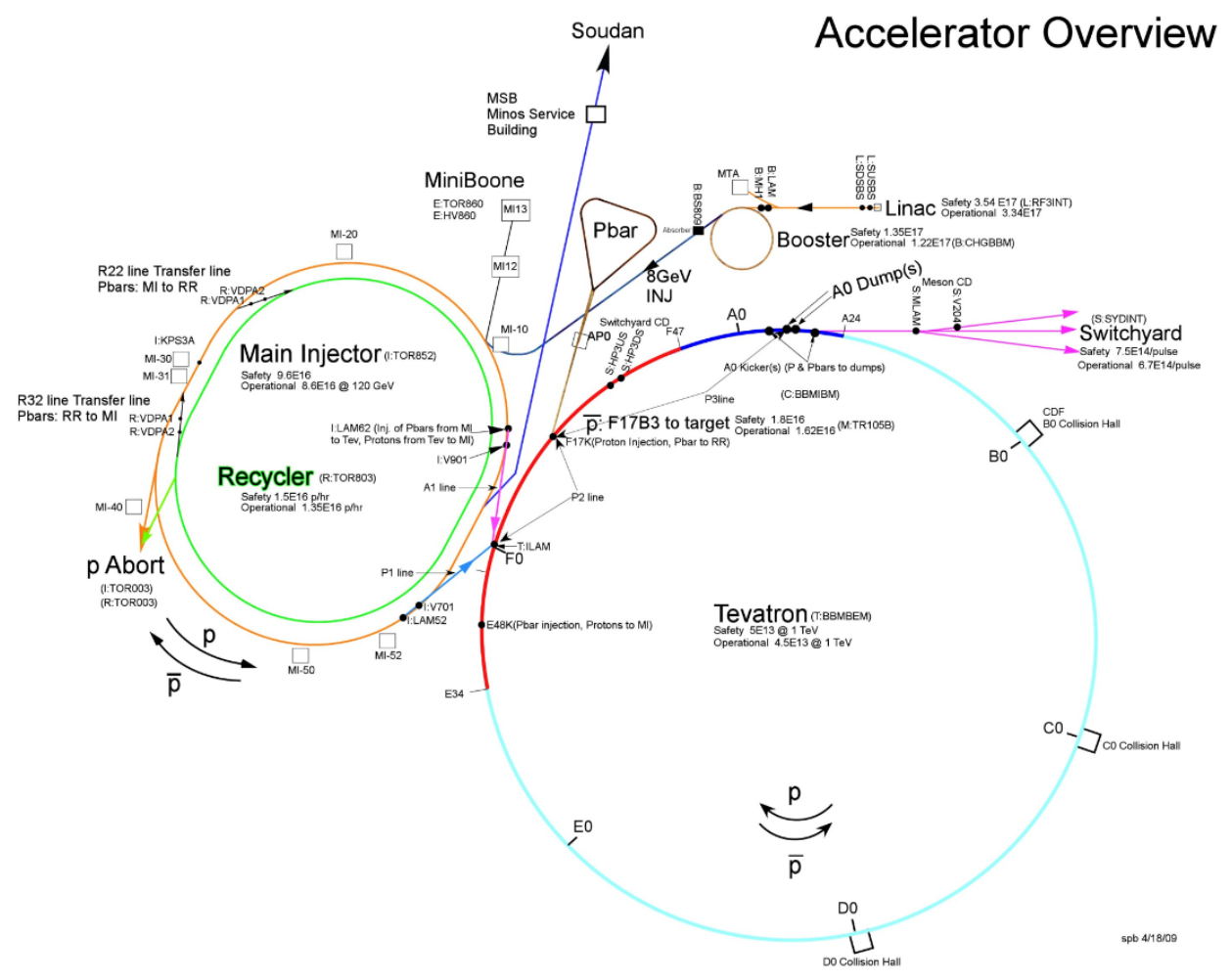

Figure 2.1: The Fermilab accelerator complex

$400 \mathrm{MeV}$ when operating in high energy physics mode. The Linac can also provide beam to the Neutron Therapy Facility, which treats certain types of cancers with a beam of neutrons. For this mode, the Linac accelerates the $H^{-}$ions to $66 \mathrm{MeV}$ before they strike a target to produce the neutron beam.[25]

Booster The $400 \mathrm{MeV}$ ions from the Linac are transferred to the Booster for further acceleration. During the transfer, they are passed through a carbon foil to strip off their electrons, yielding $\mathrm{H}^{+}$ions, or protons.[26] The protons are then accelerated to $8 \mathrm{GeV}$ and either transferred to the Main Injector or sent to the MiniBooNE target to produce a beam of muon neutrinos.

Main Injector The Main Injector is the workhorse of the Fermilab accelerator complex. The principal purpose of the accelerator is to provide $150 \mathrm{GeV}$ proton and anti-proton beams to the Tevatron. The proton beam for Tevatron injection is provided from the Booster, 
accelerated to $150 \mathrm{GeV}$, and injected into the Tevatron. The Main Injector also provides a beam of $120 \mathrm{GeV}$ protons to the Neutrinos at the Main Injector target, which produces a beam of neutrinos for the MINERvA AND MINOS experiments[27, 28]. Fixed target experiments at Fermilab can also receive a $120 \mathrm{GeV}$ proton beam from the Main Injector. The Antiproton Source also uses a $120 \mathrm{GeV}$ protons from the Main Injector to produce antiprotons, which are then injected back into the Main Injector, accelerated to $150 \mathrm{GeV}$, and injected into the Tevatron. Finally, the Main Injector can also transfer $8 \mathrm{GeV}$ antiprotons to and from the Recycler[29].

Antiproton Source The first component of the Antiproton Source is the nickel alloy target, which is struck by the proton beam from the Main Injector, producing a wide variety of particles, including proton-antiproton pairs. The target is followed by a magnet system which selects antiprotons at about $8 \mathrm{GeV}$ and guides them into the Debuncher. The Debuncher employs stochastic cooling to reduce the momentum spread before passing the beam to the Accumulator, which stores successive pulses of antiprotons while further cooling them. When enough antiprotons are stored in the Accumulator, they are transferred back to the Main Injector for storage in the Recycler[30].

The Recycler is a storage ring lying along the ceiling of the Main Injector. The original design purpose of the Recycler was to accept the remainder of the antiproton beam from the Tevatron at the end of a store, increasing the luminosity in the next store. However, after problems achieving this early in Run II, the Recycler was repurposed to store antiprotons from the Antiproton Source. This greatly increased the luminosity of the antiproton beams in the Tevatron. Like the Debuncher and the Accumulator, the Recycler uses stochastic cooling, but also employs electron cooling. A pelletron accelerates a beam of $8 \mathrm{GeV}$ electrons, which is injected into the middle of the antiproton beam in the Recycler. Because electrons are much easier to cool, the beam from the pelletron is very cold, and momentum transfer between the electron beam and the antiproton beam cools the antiproton beam, reducing its spread in both momentum and space[25]. 
Tevatron The Tevatron is the largest and highest energy accelerator at Fermilab. Protons and antiprotons at $150 \mathrm{GeV}$ are transferred from the Main Injector to the Tevatron and accelerated to $980 \mathrm{GeV}$, and then the beams are focused and crossed at two points, the locations of $\mathrm{CDF}$ and $\mathrm{D} \varnothing$. This produces high energy interactions between the protons and antiprotons, the results of which are recorded by the detectors[31].

The protons and antiprotons are arranged in the Tevatron to form two beams consisting of 36 bunches of particles. The bunches are in 3 trains of 12 bunches each, separated by a significant gap, which allows time for kicker magnets to switch on and move the beam into a beam dump when the beam is aborted. The bunch spacing within a train produces a beam crossing every $396 \mathrm{~ns}[31]$.

In order to provide a high enough magnetic field strength to bend the high energy particles around a $1 \mathrm{~km}$ radius circle, the Tevatron employs superconducting magnets. This entails maintaining the magnets at liquid helium temperatures. The Tevatron was the first synchrotron to use superconducting magnets. The Tevatron uses quadrupole and higher-order magnets to keep the beam focused, especially near the collision points, where the low- $\beta$ quadrupoles perform the final focusing for the collisions[31].

Run II of the Tevatron began on June 30, 2001, and ended on September 30, 2011. During this time, the Tevatron delivered proton-antiproton collisions with $\sqrt{s}=1.96 \mathrm{TeV}$ totalling an integrated luminosity of $11.8 \mathrm{fb}^{-1}$. Of this, CDF was able to acquire $9.9 \mathrm{fb}^{-1}$, giving a total data taking efficiency of about $84 \%$, as shown in figures 2.2 and 2.3. CDF maintained a high data taking efficiency throughout Run II, as can be seen in figure 2.3.

\subsection{The Collider Detector at Fermilab}

The Collider Detector at Fermilab is one of two particle detectors situated at collision points on the Tevatron. CDF is a 5000 ton general purpose, nearly hermetic particle detector employing silicon and drift chamber tracking inside a solenoid, a plastic scintillator sampling calorimeter, and muon detectors situated outside the steel structural members[32]. 


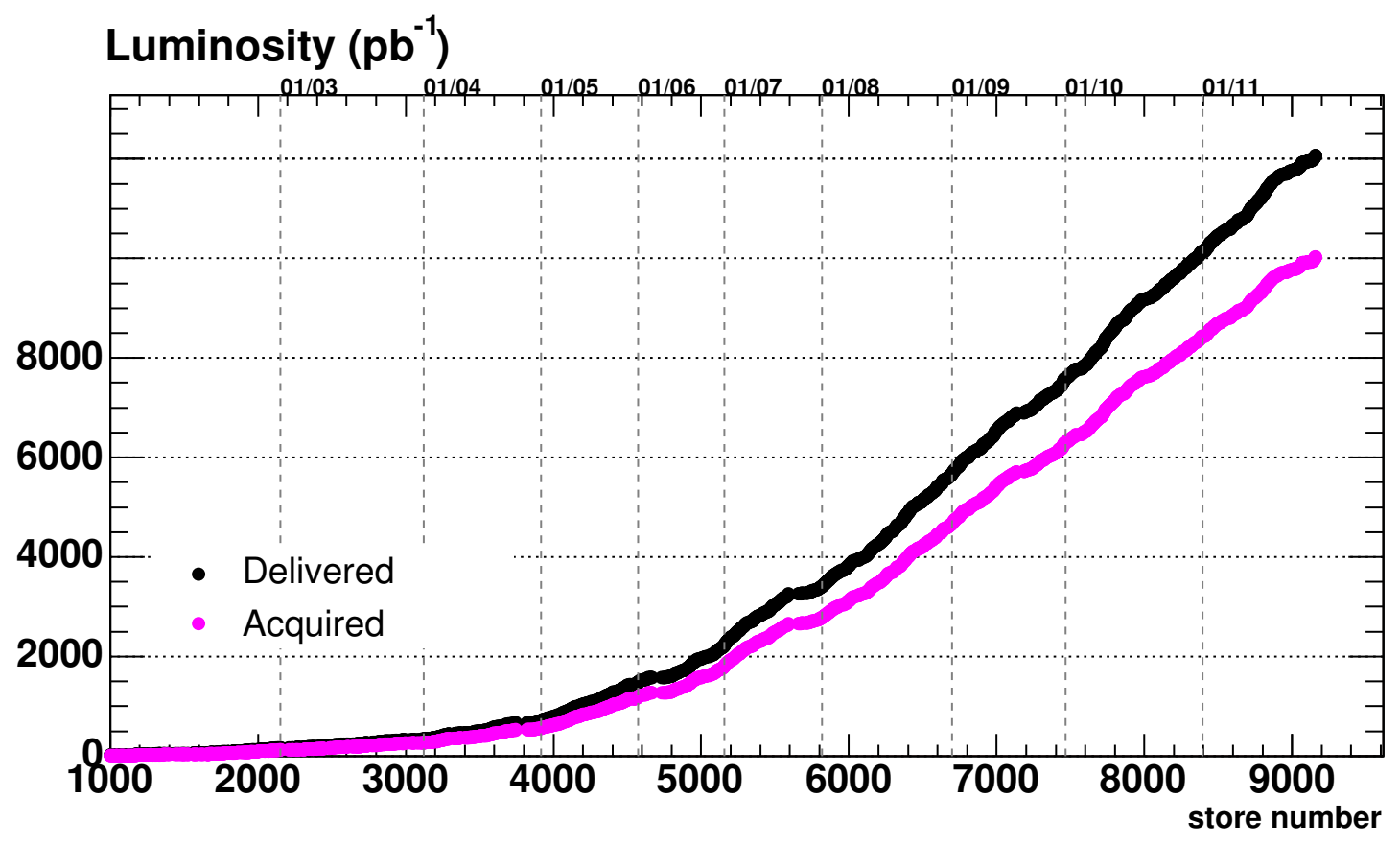

Figure 2.2: Tevatron integrated luminosity delivered to and acquired by CDF

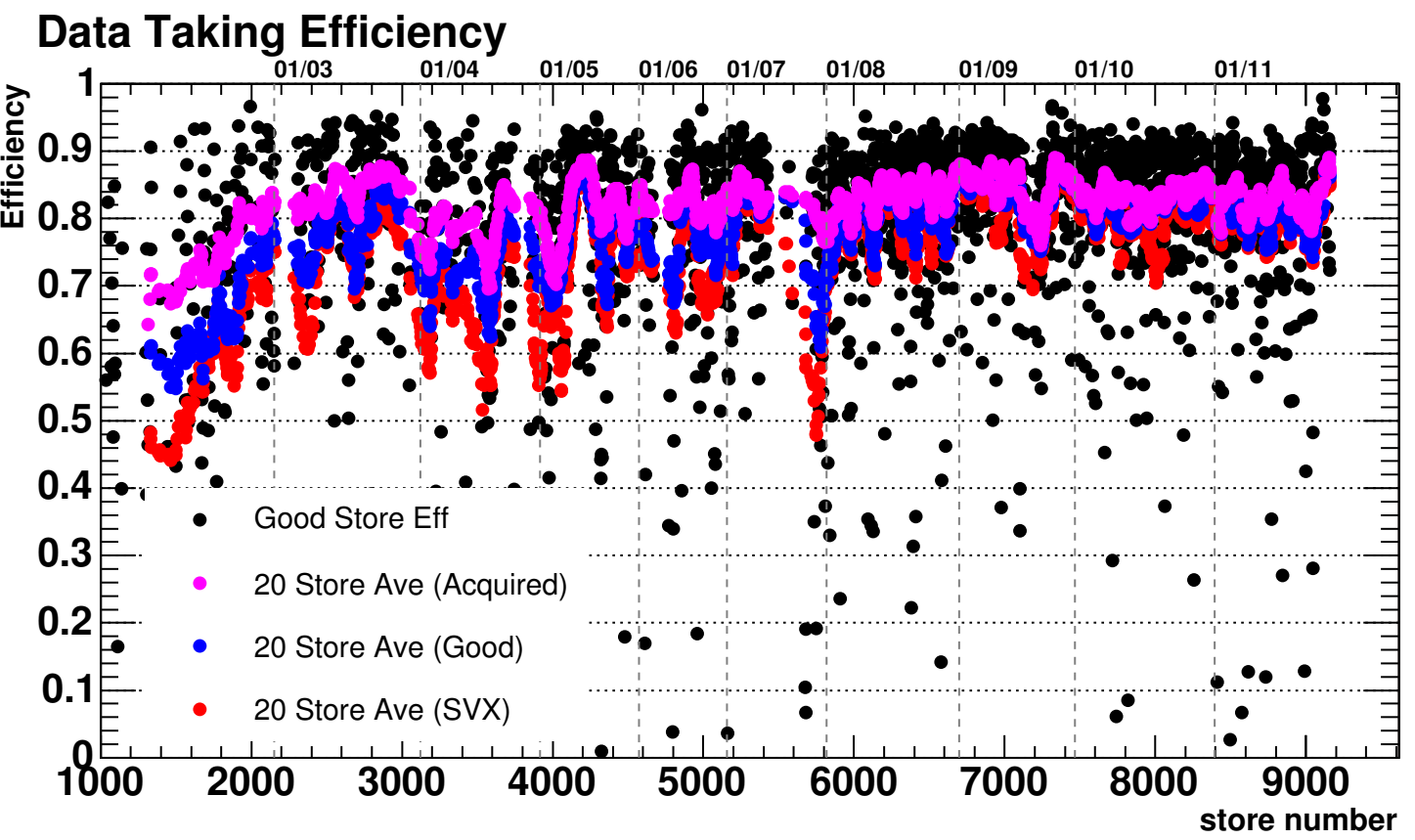

Figure 2.3: CDF data taking efficiency during Run II 


\section{CDF Tracking Volume}

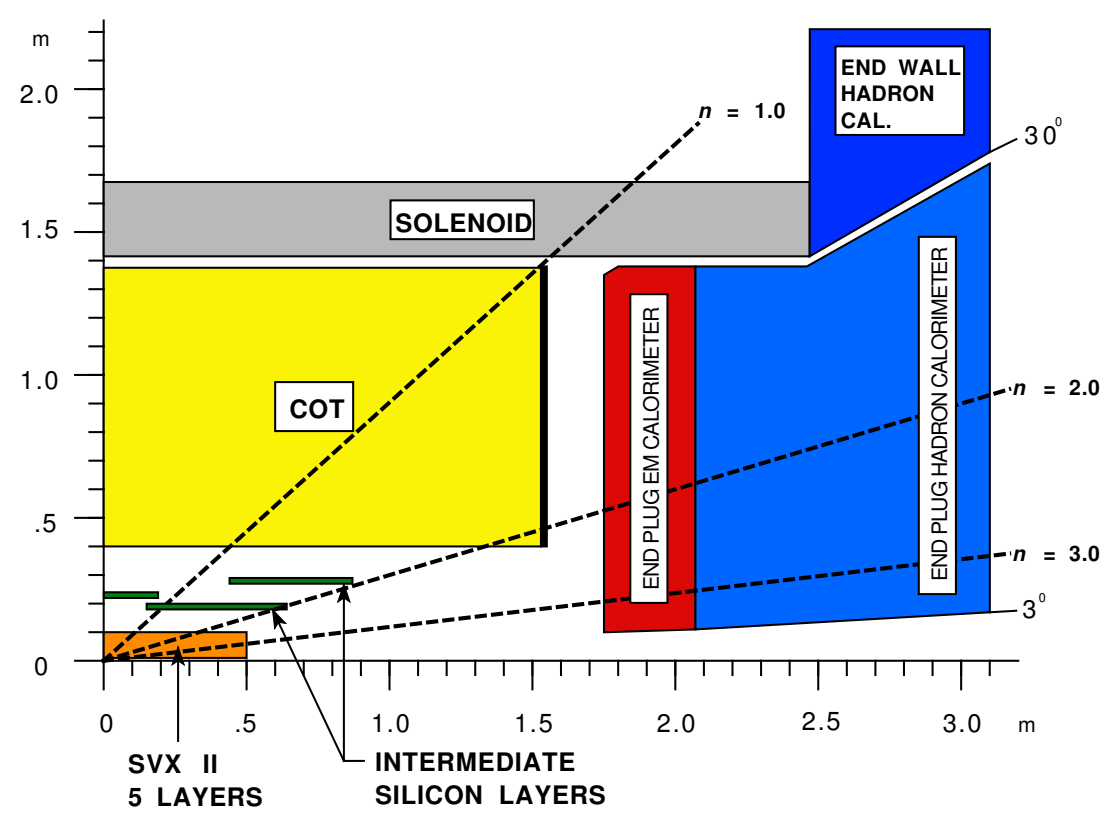

Figure 2.4: Layout of the components of the CDF tracking system

\subsubsection{Tracking}

$\mathrm{CDF}$ has a very large tracking volume contained within a $1.4 \mathrm{~T}$ solenoidal magnet field (figure 2.4). The field is provided by a superconducting solenoid $1.5 \mathrm{~m}$ in radius and $4.8 \mathrm{~m}$ in length. The solenoid is made of aluminum supported Niobium-Tin superconductor, cooled by liquid helium. Inside the solenoid are the Central Outer Tracker (COT), a large open-cell wire drift chamber, and the silicon vertex detector, a high-precision silicon strip tracker.

Central Outer Tracker The COT is $1.38 \mathrm{~m}$ in radius and provides full tracking coverage up to $\left|\eta_{\mathrm{d}}\right|<1.0$, with partial coverage up to $\left|\eta_{\mathrm{d}}\right|<2.0$. The 30240 sense wires are arranged in 12-wire cells in 8 superlayers, providing 96 tracking measurements between 48 and $131 \mathrm{~cm}$. Figure 2.5 shows the arrangement of the field-generating wires, the sense wires, and the electric field that drives the ions to the sense wires. Superlayers 2, 4, 6, and 8 are axial layers, with wires that go straight from one endplate to the other. Superlayers 1, 3, 5, and 7 are stereo layers, in which the wires are tilted at $a \sim 3^{\circ}$ in order to provide some 


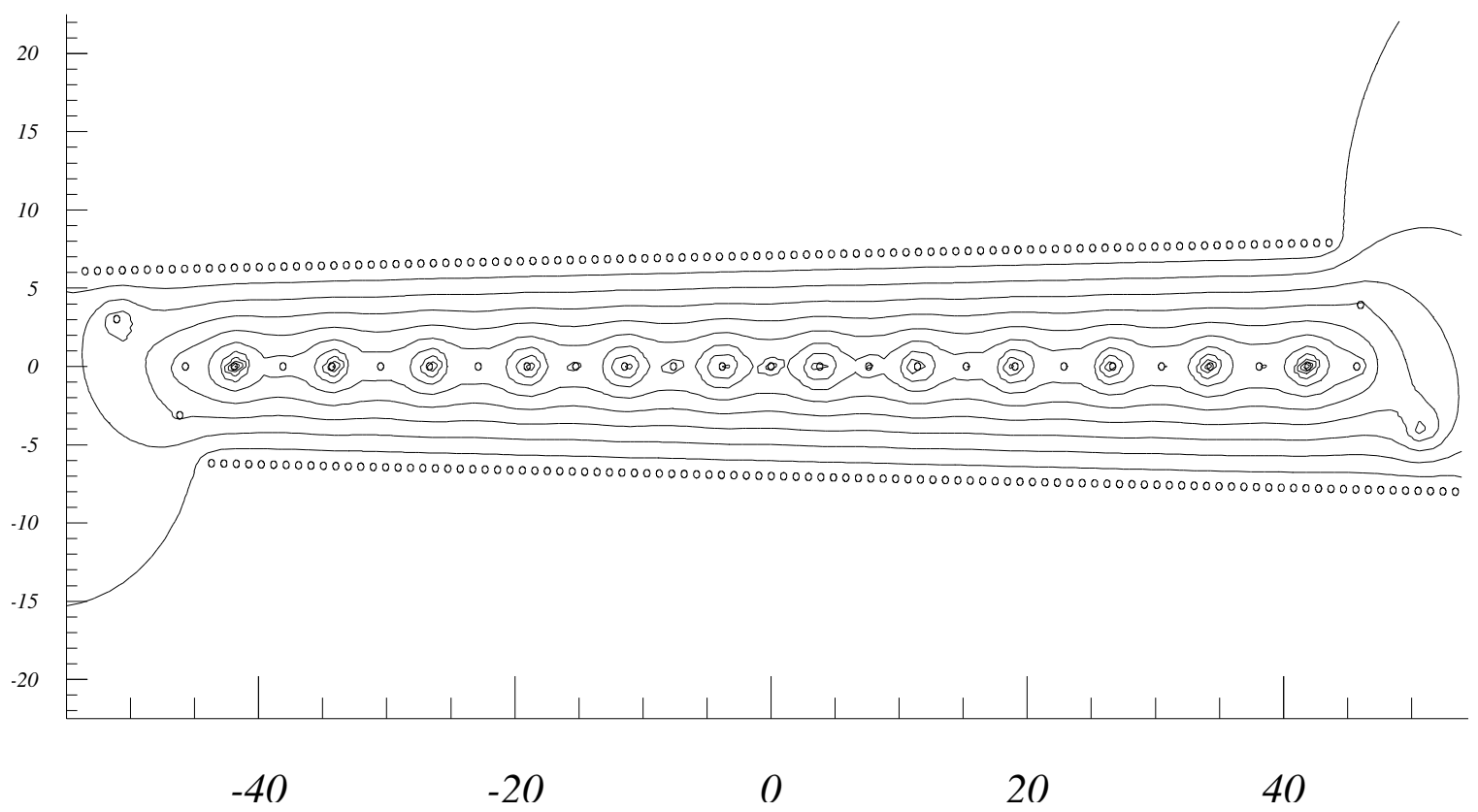

Figure 2.5: Electric equipotential contours generated by the field and sense wires in a single COT cell

information about the longitudinal direction of a track. The drift chamber is filled with a mixture of Ar, Et, $\mathrm{CF}_{4}$, and air, which provides the very short ion drift times necessary for the $396 \mathrm{~ns}$ bunch spacing. Normal atmospheric air in very small quantities was added to the gas mixture midway through Run II in order to deal with a wire aging problem. A single hit on a wire in the COT can be localized in $r-\phi$ with about $180 \mu \mathrm{m}$ resolution[32].

Silicon system The silicon tracking system is composed of two subsystems: the Silicon Vertex Detector (SVX) plus Layer 00, and the Intermediate Silicon Layers (ISL), shown in figure 2.6. The SVX is a high-precision silicon strip detector located immediately outside the beampipe, with tracking and $b$-tagging coverage out to $\left|\eta_{\mathrm{d}}\right|<2.0$. The ISL consists of some additional layers of silicon strips and occupys the space between the SVX and the COT. An end view of the layout of these detectors is shown in figure 2.6[32].

The SVX has six layers $(00,0,1,2,3,4)$ of double sided radiation-hardened silicon wafers covering from 2.44 to $10.6 \mathrm{~cm}$ from the beam. These wafers are covered on each side with strips of $p$ and $n$ type silicon with a pitch of 60 to $141 \mu \mathrm{m}$, depending on the layer and 


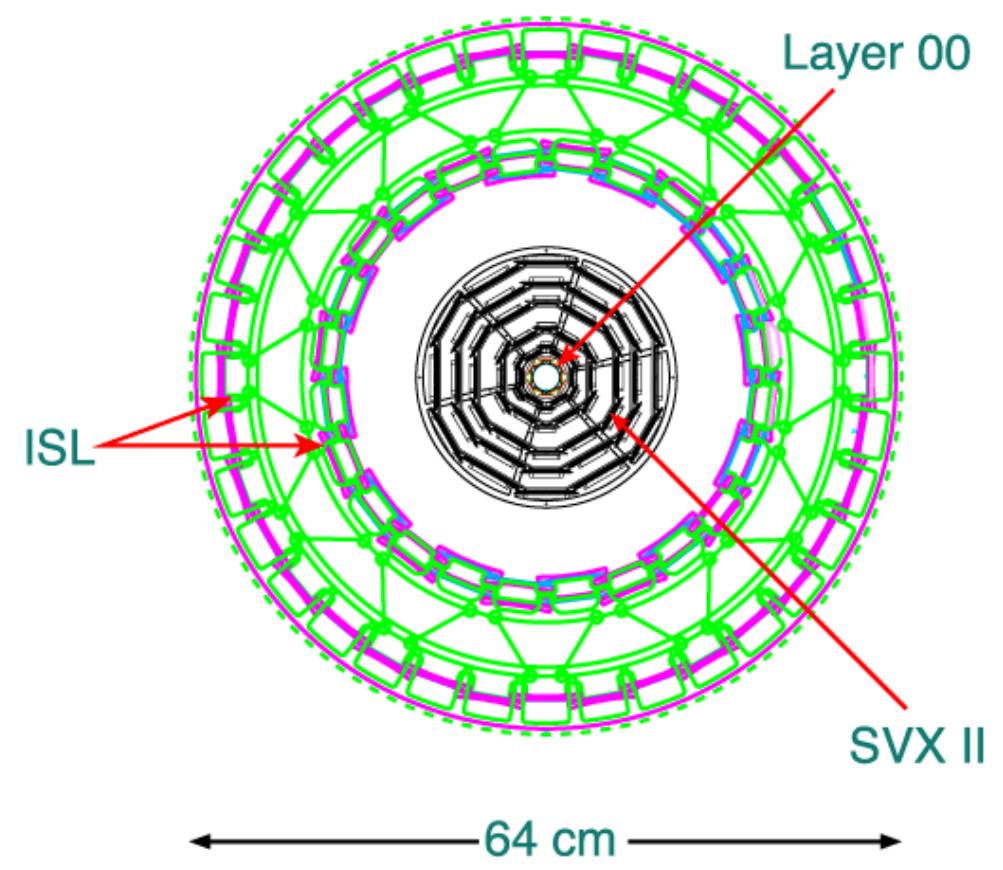

Figure 2.6: End view of the silicon system, showing the Silicon Vertex Detector (SVX) and the Intermediate Silicon Layers (ISL)

strip type. On the $p$ side, the strips run along the length of the detector, parallel to the beamline, providing $r-\phi$ detection. On the $n$ side, for layers 0,1 , and 3 , the strips run at $90^{\circ}$, around the beamline, providing $r-z$ detection. On layers 2 and 4 , the $n$ type strips are set at $1.2^{\circ}$ to the beamline, still providing some stereo resolution. Layer 00 is similar to the other 5 layers of the SVX, but consists of only single-sided silicon detectors, which allows improved radiation resistance[33, 34].

The ISL is a supplementary silicon tracking system consisting of three layers covering different pseudorapidity as shown in figure 2.7. In the central region $\left(\left|\eta_{\mathrm{d}}\right|<1\right)$, there is a single layer of silicon located $22 \mathrm{~cm}$ from the beamline. In the forward region $\left(1.0<\left|\eta_{\mathrm{d}}\right|<2.0\right)$, there are two layers, at 20 and $28 \mathrm{~cm}$ from the beamline. These layers consist of double sided silicon wafers with small angle stereo tracking similar to layers 2 and 4 of the SVX. The pitch of the strips is similar, at $55 \mu \mathrm{m}$ (axial) and $73 \mu \mathrm{m}$ (stereo), but the readout system combines every two strips into one readout channel, reducing the necessary readout bandwidth[32].

The central single layer provides additional tracking to supplement the low-resolution 


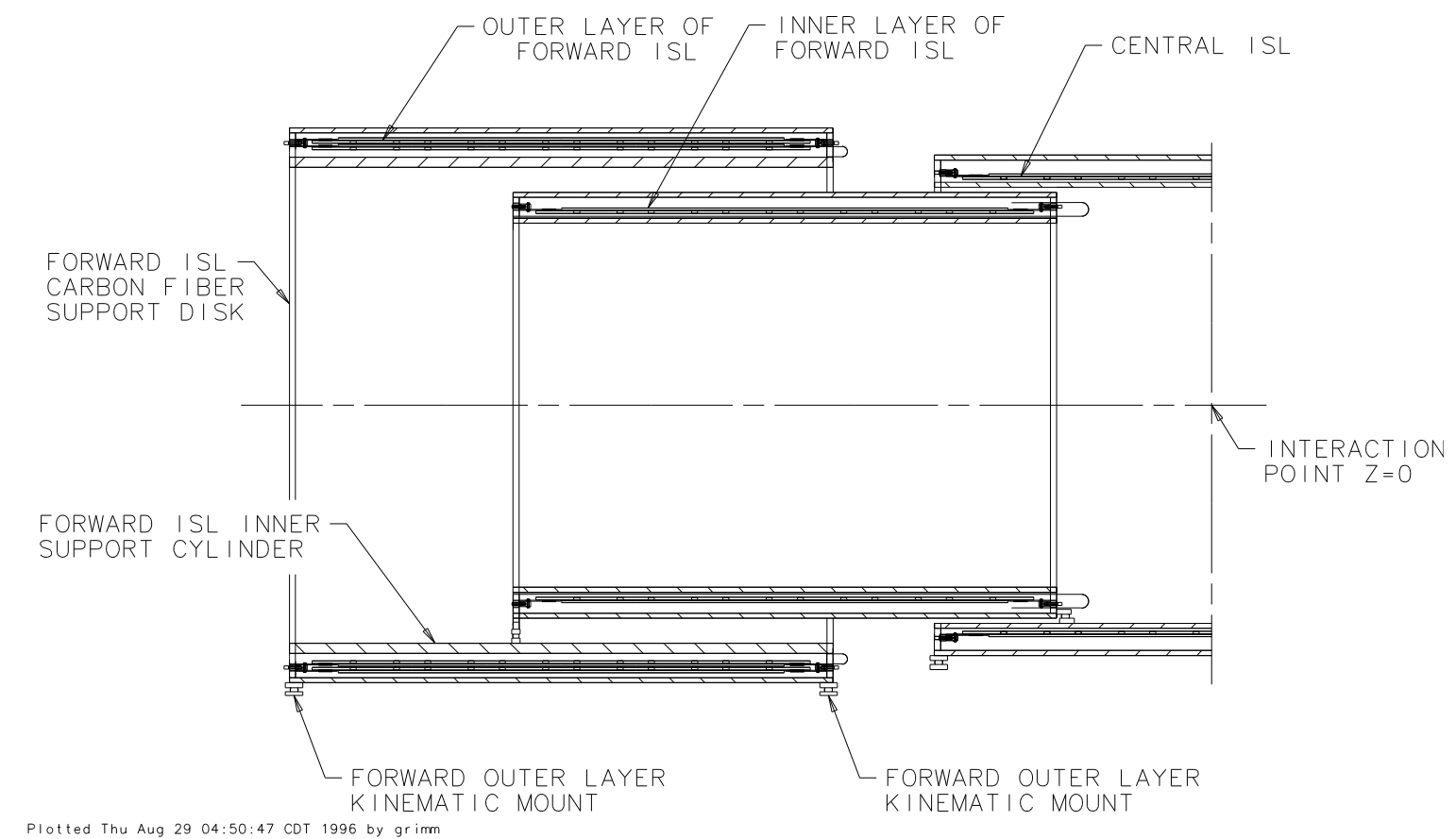

Figure 2.7: Cut-away view of the layout of the Intermediate Silicon Layers (ISL)

COT tracks and the high-precision, high-occupancy tracking environment of the SVX. This helps to reduce tracking inefficiencies by opening up the possibility of finding tracks in the silicon system and extrapolating them into the COT, rather than the other way around. The double forward layer extends $3 \mathrm{~d}$ track reconstruction to the regions only partially covered by the COT, and brings high-precision tracking and $b$-tagging to the plug region[32].

Combined tracking performance The Solenoid+COT+ISL+SVX combined tracking system enables very high precision determination of the momentum of charged particles. The large tracking volume provides sufficient tracking hits over a sufficient distance to determine the helix parameters of the tracks very precisely. The high magnetic field gives all but the very highest momentum tracks enough curvature to distinguish different momenta.

Using the COT alone, we obtain a curvature measurement with a resolution of approximately $0.68 \times 10^{-4} \mathrm{~cm}^{-1}$, leading to a transverse momentum resolution $\delta p_{T} / p_{T}^{2} \simeq$ $0.3 \% \mathrm{GeV}^{-1}$. The residuals for the measurement of the helix parameters of simulated COT tracks is shown in figure 2.8. The COT finds tracks with an efficiency of about $95 \%$ anywhere 

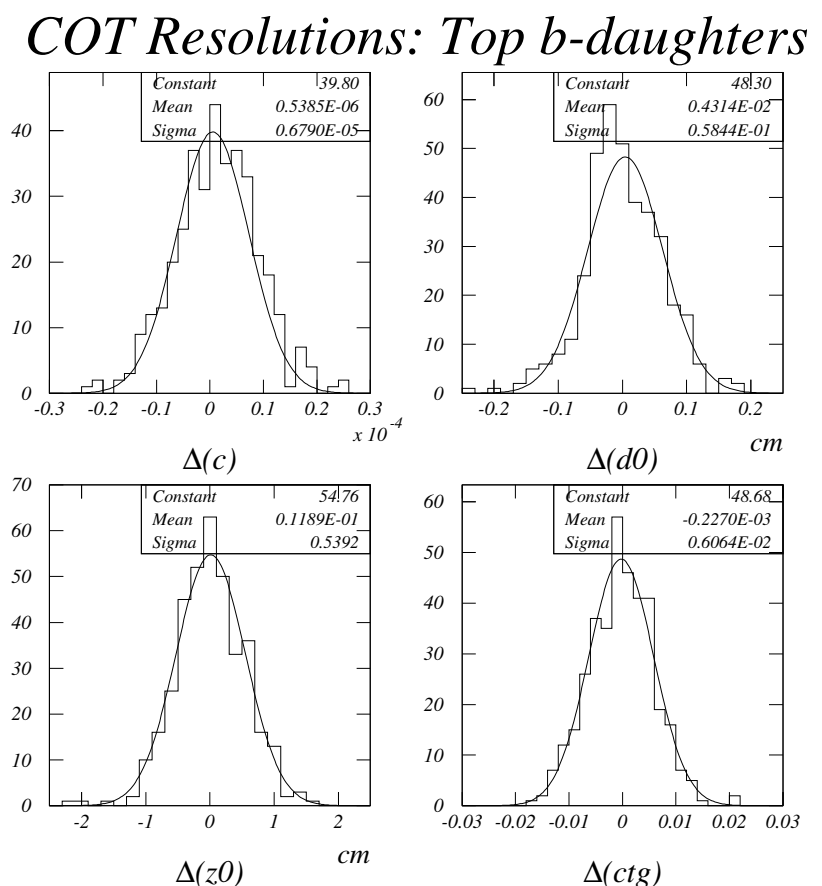

Figure 2.8: Residuals for helix parameters (curvature, impact parameter, $z_{0}$ and $\cot \theta$ ) in COT

within $\left|\eta_{\mathrm{d}}\right|<1.0[32]$.

In addition to measuring track helix parameters, the silicon system excels at finding and precisely measuring vertices, which is crucial to the detector's $b$-tagging performance. The SVX+ISL impact parameter resolution is $\delta d_{0}=15 \mu \mathrm{m}$, allowing excellent primary and secondary vertex reconstruction. The resolution on other track parameters is similar to the COT alone[32].

Tracks reconstructed in the silicon system can be used to point into the COT to seed the track reconstruction algorithm there. Alternatively, hits in the silicon system can be attached to an already reconstructed COT track. This dual method increases both the resolution and the track-finding efficiency of the entire system beyond what either system would be capable of independently. The combined resolution for $b$-daughter track parameters in simulated $t \bar{t}$ events is shown in figure 2.9. 


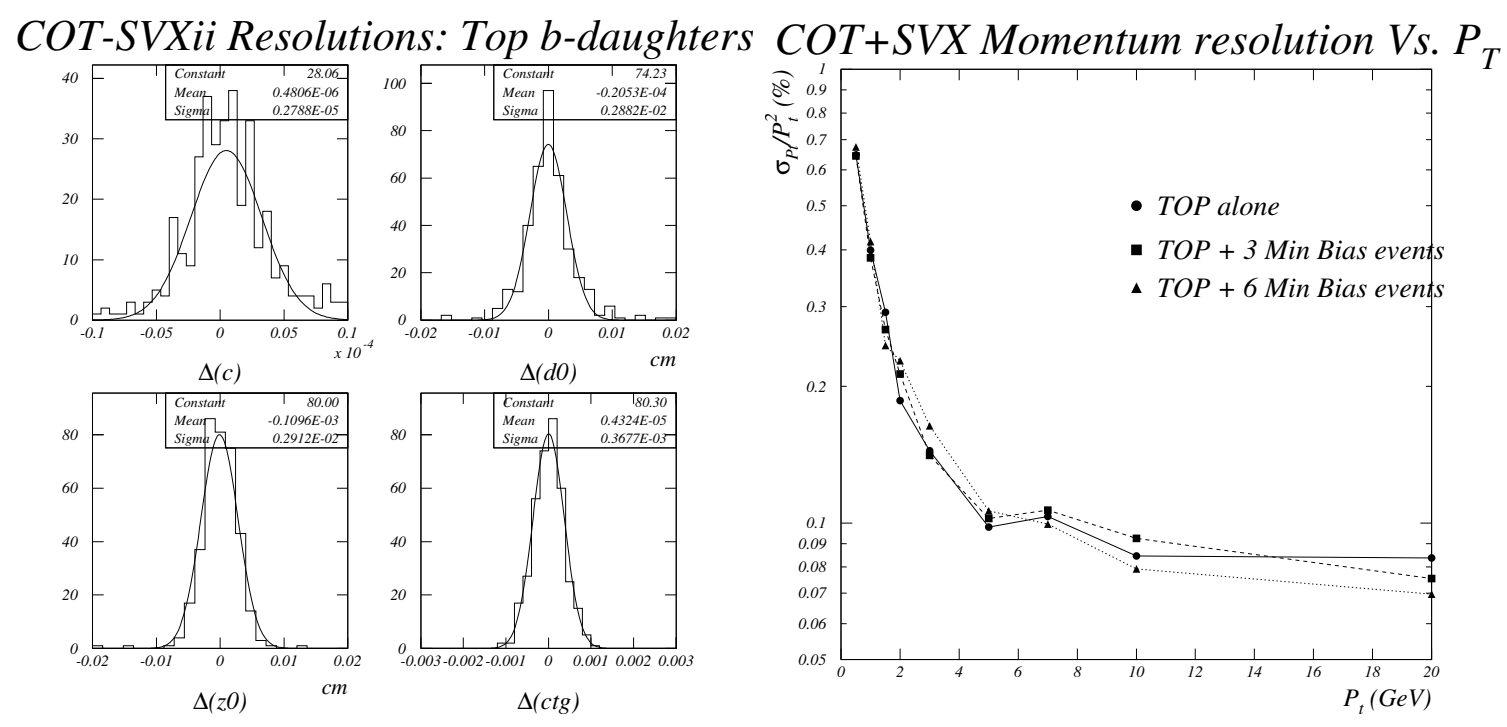

Figure 2.9: Helix parameter residuals and transverse momentum resolution of the combined tracking system

\subsection{Calorimetry}

The CDF calorimeter is divided into two regions, central and plug, each of which contains both an electromagnetic and a hadronic portion. The central calorimeter covers $\left|\eta_{\mathrm{d}}\right|<1.1$, with partial coverage supplemented by the end-wall hadronic calorimeter up to $\left|\eta_{\mathrm{d}}\right|<\sim 1.4$. The plug calorimeter covers the portion of the detector close to the beamline, $1.3<\left|\eta_{\mathrm{d}}\right|<3.6$. The central and plug calorimeters both consist of two distinct segments, an electromagnetic calorimeter and a hadronic calorimeter.

The electromagnetic calorimeter is composed of alternating layers of lead absorber and plastic scintillator. The high- $Z$ lead causes electrons and photons to undergo Bremsstrahlung and to pair produce an electron and positron, respectively. This successive production of lower energy particles is referred to as the shower. As the particles in the shower pass through the scintillator tiles, they ionize atoms, which decay back to their ground state by emitting a photon. These photons are collected by wavelength-shifting light pipes, which produce several low-energy photons for each incoming high-energy photon, and directed to photomultiplier tubes. The PMTs turn the scintillator light into an electrical pulse, which is 


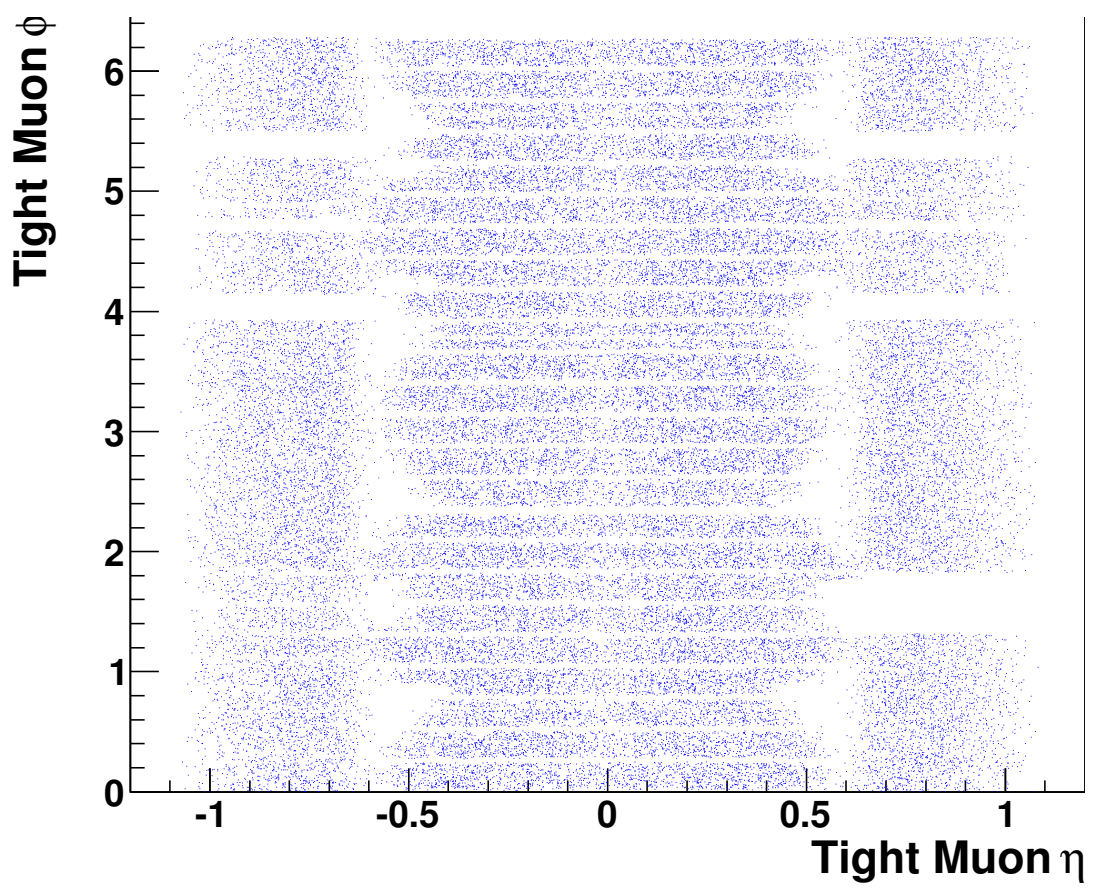

Figure 2.10: Location of tight muons in the detector

shaped, amplified, digitized, and recorded. The energy of an electron or photon incident on the electromagnetic calorimeter is roughly proportional to the total amount of light collected.

The hadronic calorimeter also uses plastic scintillator tiles, but instead of lead, steel absorber plates are used. The hadronic calorimeter is much larger than the electromagnetic calorimeter, and its principal purpose is to contain and measure a hadronic shower as completely as possible.

\subsection{Muon system}

The central muon detectors are outside the calorimeter, which absorbs nearly all electron, photon, and jet shower particles. The CMU is between the hadronic calorimeter and the steel magnet return yoke for the solenoid. The yoke provides further shielding for the CMP and the CMX. Because muons are minimum-ionizing particles at typical muon momenta, do not undergo very much Bremsstrahlung, and do not interact hadronically, they leave a track in the tracking system, a minimal energy deposit in the electromagnetic and hadronic 
calorimeters, and pass through the steel shielding. Very little else is able to penetrate the shielding, so a signal in the detectors outside the shielding is almost certainly a muon. In some jets, the shower retains enough energy that some pions escape the back of the hadronic calorimeter and leave a signal in the CMU, but it is very rare for these particles to additionally penetrate the steel shielding.

The muon detectors consist of several separate detectors. The CMU and CMP cover the central region, $\left|\eta_{\mathrm{d}}\right|<0.6$. The CMX covers the intermediate region $0.6<\left|\eta_{\mathrm{d}}\right|<1.0$. The IMU, and BMU cover the forward regions with $\left|\eta_{\mathrm{d}}\right|>1.0$. The coverage in $\eta-\phi$ space of the CMU, CMP, and CMX is shown in figure 2.10.

The CMU consists of a layer of wire chambers in a cylinder surrounding the detector. The wire chambers are contain four layers of wires and reside behind 5.5 interaction lengths of steel absorber. The CMP is outside the CMU with more steel shielding separating the two. The CMP is arranged in four large panels covering the top, sides, and bottom of the detector, and includes a similar arrangement of wire chambers along with a layer of scintillation counters. The CMX consists of large conical arches of drift tubes and scintillation counters and extends the coverage of the central muon system. The forward muon detectors are constructed similarly, with drift tubes and scintillation counters behind steel shielding.

The four-wire thick construction allows a very short track (a "stub") to be reconstructed, which reduces the backgrounds by eliminating spurious signals that do not form such a track, or form a track that does not point back towards the interaction point.

\subsection{Trigger and Data acquisition}

In order to use the detector measurements in an analysis, events must be saved to long term storage. This is accomplished by means of the front-end electronics systems which read signals out of the various detector components, and the trigger system which decides which events to save.

The actual detector readout is accomplished by a variety of circuits which record, transform, and digitize the analog signals from the silicon strips, tracking wires, calorimeter 


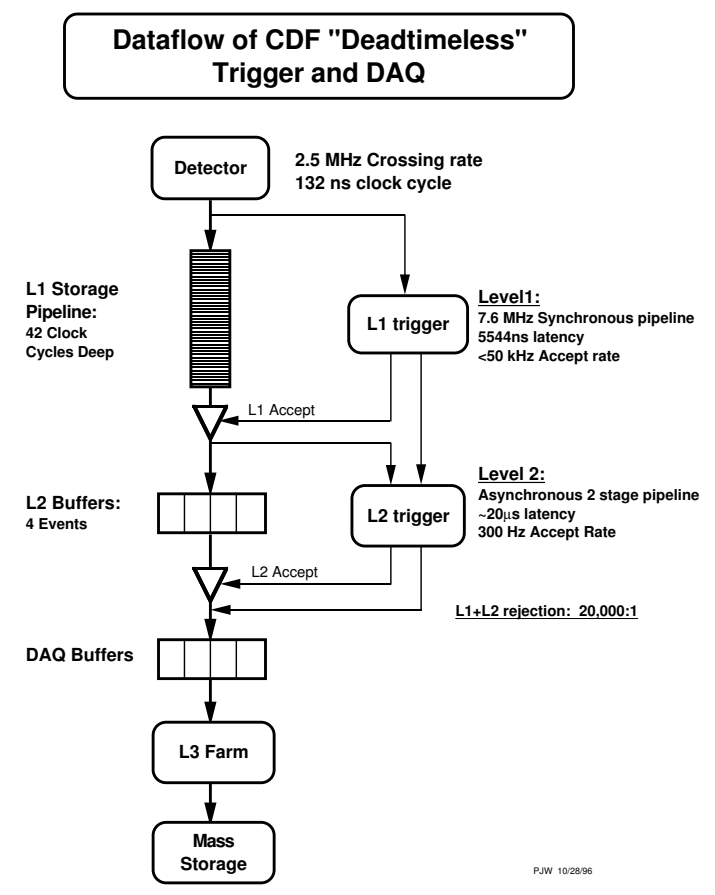

Figure 2.11: Flow of data through the CDF data acquisition and trigger system

\section{RUN II TRIGGER SYSTEM}

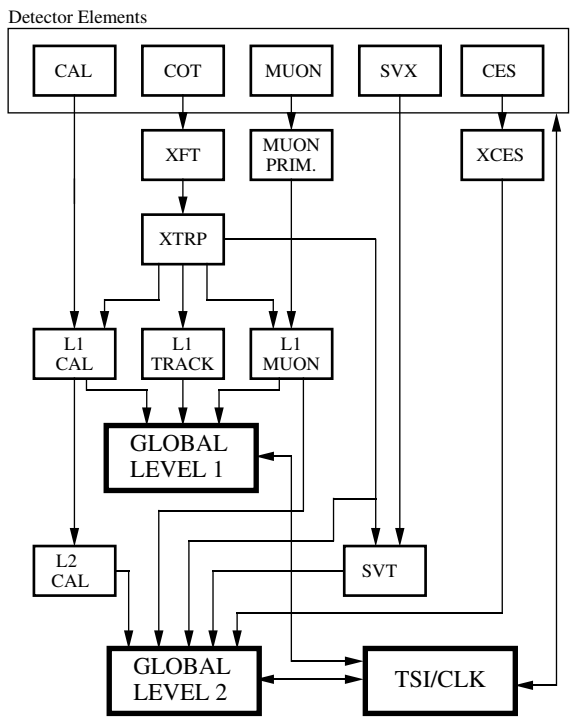

PJW 9/23/96

Figure 2.12: Data flow through the subcomponents of the trigger system 
photomultiplier tubes, etc. The silicon tracking system is read out by radiation-hard ASICs that are physically co-located with the silicon sensors themselves inside the detector. The COT is read out through Amplifier/Shaper/Discriminator chips which process the raw analog signal from the wires into a form that is usable by the analog-to-digital converters, and the muon system readout is similar. The calorimeter readout is based on the Charge Integrating and Encoding (QIE) chip, which outputs the amount of charge collected by the PMTs as a digital signal[32].

The trigger is an integral part of the data acquisition system, and is tightly integrated with the detector readout. Bunch crossings occur at a rate of $2.5 \mathrm{MHz}$, and this must be reduced to less than $100 \mathrm{~Hz}$ to write the events to long-term storage. This is accomplished with a multi-level system of fast electronics and computers, outlined in figure 2.11.

Level 1 consists of fast electronics which perform a simple but synchronous reconstruction of an event. The eXtremely Fast Tracker identifies COT tracks in three dimensions and coarsely measures their transverse momentum before passing them to the XTRP, which extrapolates the tracks to the calorimeter and the muon chambers. The L1 Muon system identifies stubs in the muon chambers and matches them to extrapolated XFT tracks. The L1 Cal system finds high energy towers and correlates them with high momentum XFT tracks, as well as summing the transverse energy of all towers to find the $\mathbb{E}_{T}$. The Global Level 1 decision is made based on calorimeter towers, $\mathbb{E}_{T}$, muon candidates, and tracks and reduces the event rate from the $2.5 \mathrm{MHz}$ bunch crossing rate to less than $50 \mathrm{kHz}$.

Events which pass Level 1 are passed to Level 2, which performs a somewhat more careful event reconstruction asynchronously. The L2 Cal performs a simple cluster finding algorithm on calorimeter towers, which allows the Level 2 trigger to identify jets, electrons, and photons. This information is correlated with cluster finding in the shower maximum detectors to help reduce electron and photon trigger rates while maintaining signal acceptance. The Silicon Vertex Tracker trigger reconstructs the impact parameter of silicon tracks, seeded by XFT tracks, with high precision, which allows Level 2 to trigger on displaced tracks from $B$ hadron decays. All of the Level 1 and Level 2 trigger information is passed to the Global Level 2 decision hardware. The Level 2 trigger further reduces the $50 \mathrm{kHz}$ Level 1 event 
rate to $\sim 300 \mathrm{~Hz}$. The flow of information through all of the Level 1 and Level 2 trigger components is shown in figure 2.12 .

After passing the Level 1 and Level 2 triggers, the events are written to hard disks and processed by the Level 3 trigger, a computer farm which performs a full event reconstruction and applies the final trigger decisions. Events which pass Level 3 are fully reconstructed offline and written to archival tape. These events form the CDF dataset.

In order to drastically reduce deadtime, the CDF trigger and data acquisition system employs a pipeline and buffer technique. The readout circuits and Level 1 electronics all contain a pipeline of 42 Level 1 buffers, which store each event in sequence to give the Level 1 trigger time to process the event. At the end of the pipeline, the Global Level 1 decision either rejects the event, in which case it is lost, or accepts it, in which case it is copied to one of the four Level 2 buffers. The Level 2 buffers are also present on all readout, Level 1, and Level 2 components, and store the event indefinitely while waiting for the asynchronous Level 2 decision. In the case that an event is accepted by Level 1, but all four Level 2 buffers are filled, the event is lost to deadtime. If an event is accepted by Level 2, the event is written to a temporary storage area on a hard disk array for Level 3 processing[32]. 


\section{Chapter 3 Event SeleCtion}

Because $t \bar{t} H$ production has two $W$ bosons and up to four $b$ quarks in the final state as shown in figure 3.1, we select a sample of events containing a charged lepton and missing transverse energy, from the decay of one $W$ boson, and a large number of $b$-tagged jets. This sample is largely composed of events in which a pair of top quarks was produced, and is expected to contain a large fraction of the available $t \bar{t} H$ signal.

\subsection{Lepton ID}

Charged leptons consist of electrons and muons; because tau leptons decay before reaching the detector, it is more difficult to adequately reconstruct and identify events involving a tau lepton, and so we do not directly consider them, although events in which a $\tau$ decays to an electron or muon do enter our event sample. Electron candidates are reconstructed by identifying a high- $p_{T}$ track in the tracking system which is associated with a cluster of energy deposited in the calorimeter. Electron candidates are further refined by requiring that the calorimeter deposit and track be consistent with those expected from an electron. The variables involved in this for electron candidates in the central calorimeter (CEM electrons) are the cluster shape as measured by the shower max detector $\left(L_{\mathrm{shr}}\right.$ and $\left.\chi_{\text {strips }}^{2}\right)$, the distance between the track direction and the center of the cluster $(Q \cdot \Delta x,|\Delta z|)$, the ratio of the amount of energy deposited immediately surrounding the cluster to the cluster energy (Isol), the ratio of the cluster energy to the track momentum $(E / P)$, and the ratio of the energy deposited in the hadronic calorimeter to that deposited in the electromagnetic calorimeter 


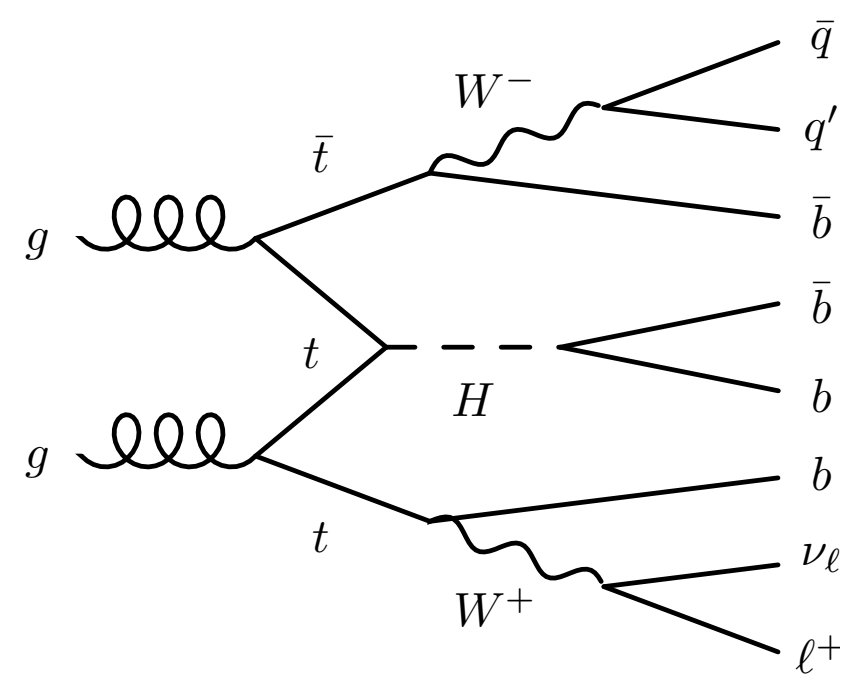

Figure 3.1: Production of $t \bar{t} H$, with a charged lepton, a neutrino, four $b$ quarks and two light quarks in the final state

\begin{tabular}{|c|c|}
\hline \multicolumn{2}{|r|}{ Electrons } \\
\hline Central (CEM) & $\left|\eta_{\mathrm{d}}\right|<1.0$ \\
\hline$L_{\mathrm{shr}}$ & $<0.2$ \\
\hline$\chi_{\text {strips }}^{2}$ & $<10$ \\
\hline$Q \cdot \Delta x$ & $>-3.0 \mathrm{~cm},<1.5 \mathrm{~cm}$ \\
\hline$|\Delta z|$ & $<3.0 \mathrm{~cm}$ \\
\hline Isol & $<0.1$ \\
\hline$E / P$ & $<2.0$ \\
\hline$E_{\text {had }} / E_{\mathrm{EM}}$ & $<0.055+0.00045 \mathrm{GeV}^{-1} \cdot E$ \\
\hline Plug (PHX) & $1.2<\left|\eta_{\mathrm{d}}\right|<2.0$ \\
\hline$\chi_{\mathrm{PEM}}^{2}$ & $<10$ \\
\hline PES 5x9 UV & $>0.65$ \\
\hline$N_{\text {Si hits }}$ & $>3$ \\
\hline Isol & $<0.1$ \\
\hline$E_{\text {had }} / E_{\mathrm{EM}}$ & $<0.05$ \\
\hline
\end{tabular}

Table 3.1: Electron identification cuts 

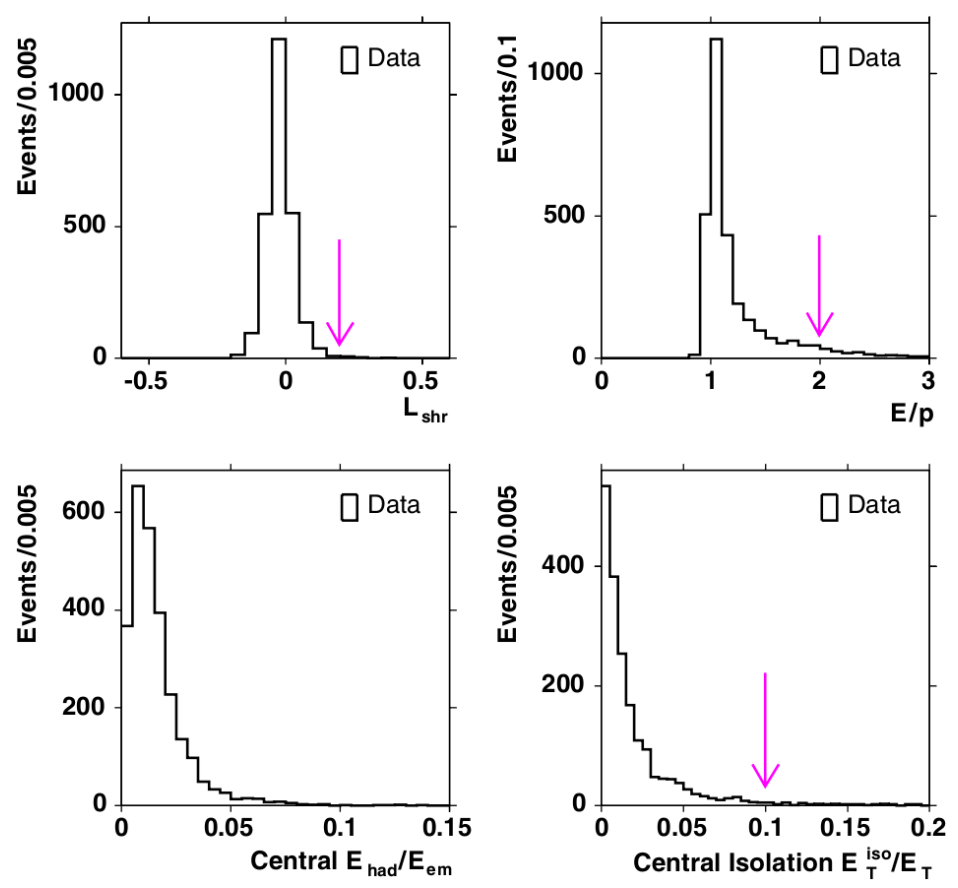

Figure 3.2: Distributions and cut locations for central electron identification variables

$\left(E_{\text {had }} / E_{\mathrm{EM}}\right)$. These cuts are summarized in table 3.1, and the distributions of some of these variables are shown in figure $3.2[35]$.

For electron candidates that are in the forward, or plug, region of the detector, the electron does not pass through the entire tracking system. As a result, tracks are reconstructed using an alternate algorithm that uses the direction of an isolated calorimeter deposit as a seed, and searches for signals in the tracking system consistent with a track pointing to that deposit. This is called Phoenix tracking, and the electron candidates are called Pheonix or PHX electrons. Because the detector components are different in this region, different variables are used for electron identification. There are two shower shape variables used, $\left(\chi_{\mathrm{PEM}}^{2}\right.$ and PES 5x9 UV). To ensure that the track is well reconstructed, we also examine the number of hits in the tracking system $\left(N_{\mathrm{Si}}\right.$ hits $)$. The isolation and hadronic-electromagnetic energy ratio are also used ( $I$ sol and $\left.E_{\text {had }} / E_{\mathrm{EM}}\right)$. These cuts are summarized in table 3.1 and the distributions of some of these variables are shown in figure 3.3[35].

Muon candidates are similarly reconstructed by identifying a high- $p_{T}$ track which is 

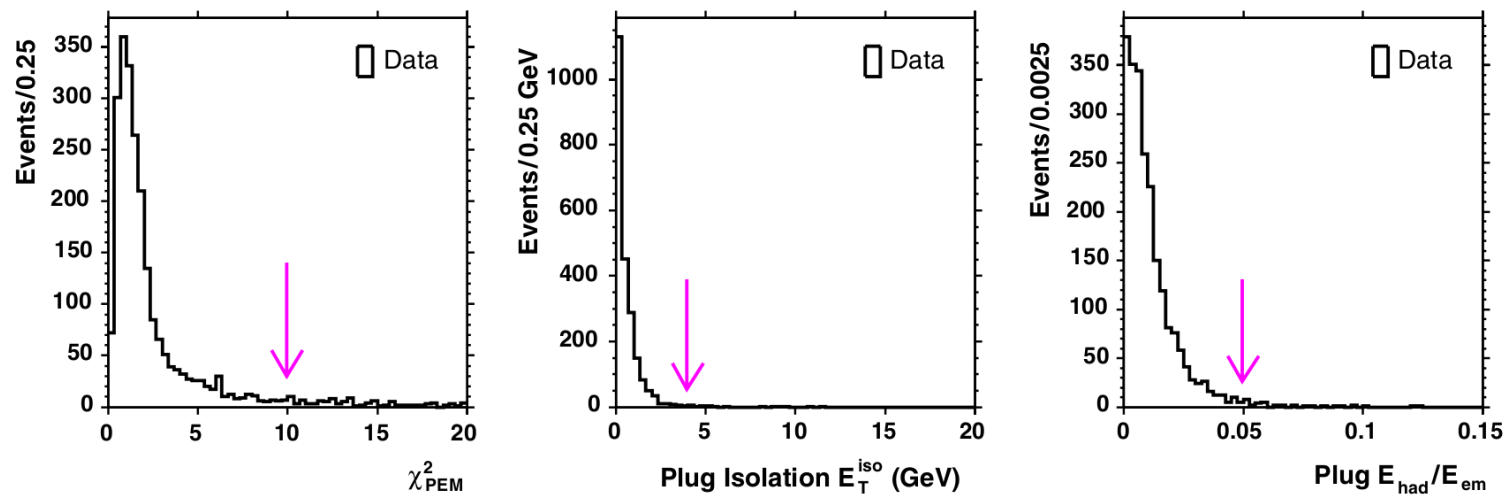

Figure 3.3: Distributions and cut locations for plug electron identification variables

\begin{tabular}{rr}
\hline \multicolumn{3}{c}{ Muons } \\
\hline CMUP or CMX & $\left|\eta_{\mathrm{d}}\right|<1.0$ \\
$E_{\text {had }}$ & $<6 \mathrm{GeV}$ \\
$E_{\mathrm{EM}}$ & $<2 \mathrm{GeV}$ \\
$N_{\text {Ax seg }}$ & $\geq 3$ \\
$N_{\text {St seg }}$ & $\geq 2$ \\
$d_{0}$ (with Si hits) & $<0.02 \mathrm{~cm}$ \\
$d_{0}$ (no Si hits) & $<0.2 \mathrm{~cm}$ \\
Isol & $<0.1$ \\
\hline
\end{tabular}

Table 3.2: Muon identification cuts 


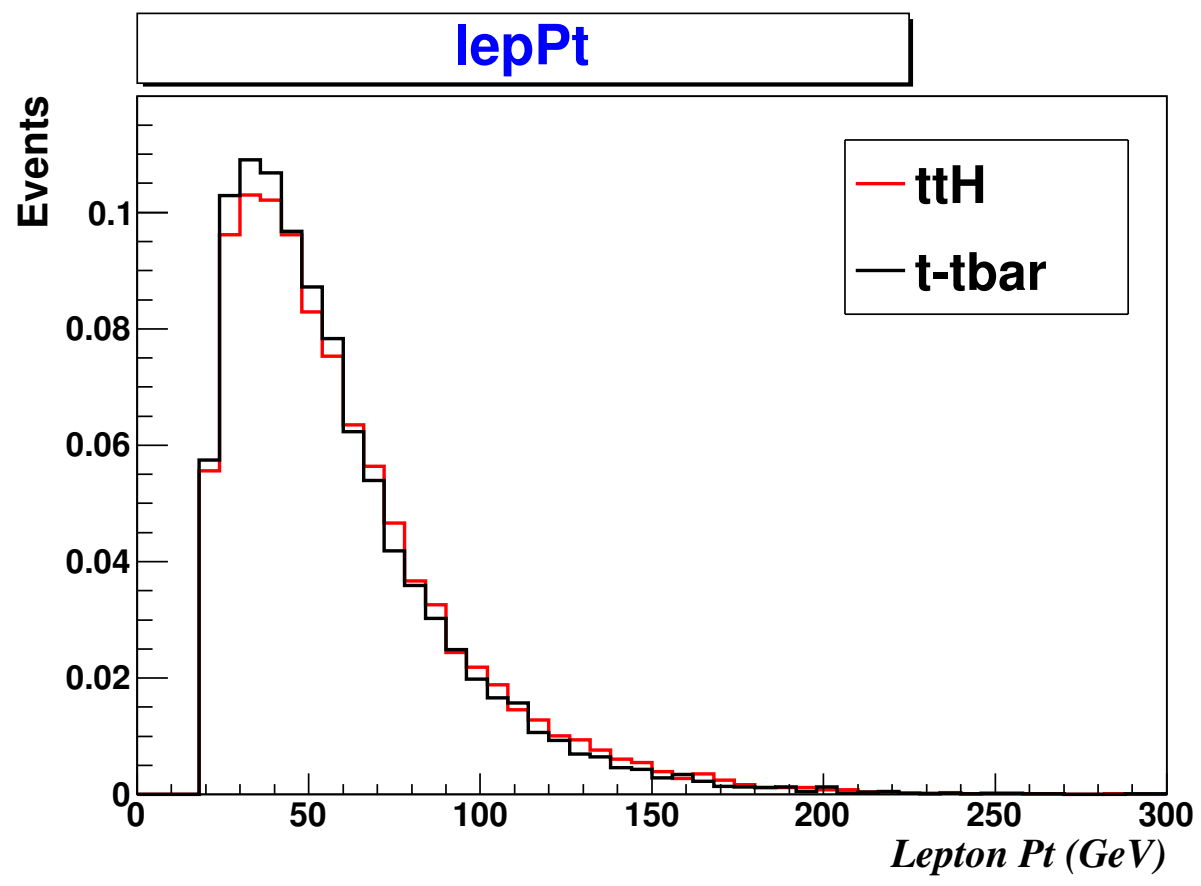

Figure 3.4: Lepton transverse momentum in $t \bar{t}$ and $t \bar{t} H$

associated with a small energy deposit in the calorimeter and a signal in the muon detectors. In this analysis, we consider muons that enter both the CMU and CMP detectors (CMUP muons) and muons that enter the CMX detector (CMX muons). These detectors cover the detector out to $|\eta|<1.0$. The identification cuts for CMUP and for CMX muons are the same. The energy deposited in the both the hadronic and electromagnetic calorimeters must be consistent with that expected from a minimum-ionizing particle ( $E_{\text {had }}$ and $\left.E_{\mathrm{EM}}\right)$. The track must be well-reconstructed in multiple axial and stereo layers of the tracking chamber ( $N_{\mathrm{Ax} \mathrm{seg}}$ and $\left.N_{\mathrm{St} \text { seg }}\right)$. The impact parameter of the track must be small, although for tracks with no signal in the silicon tracker, this cut is loosened due to poorer track resolution $(D 0)$. Finally, the ratio amount of energy deposited in the calorimeter near the track to the track momentum must be small ( I sol). These cuts are summarized in table 3.2[35].

Cosmic ray muons passing through the detector produce signals in our detector that we sometimes incorrectly reconstruct as muons originating from the proton-antiproton collision. Because these are real muons, they do leave signals in the muon detectors, minimum-ionizing 
deposits in the calorimeters, and high momentum tracks that are well matched to the muon detector signals. These appear in our reconstruction software as back-to-back muons with a high invariant mass, but often with very large impact parameters, and with tracker timing that does not match a bunch crossing very well. So, we remove events containing cosmic rays in this analysis via three different cuts. First, since we require only single leptons, the presence of two reconstructed muons will prevent the majority of cosmic ray events from entering our analysis. Second, the track impact parameter cut discussed above removes the majority of the remaining cosmic ray events. Finally, we require that the tracker system timing information match a bunch crossing. After this threefold filtering process, cosmic ray muons do not have a significant impact on our analysis[35].

We also require that all leptons have a transverse momentum of at least $20 \mathrm{GeV}$ and a track $z_{0}$ less than $60 \mathrm{~cm}$. The $p_{T}$ cut is driven by our triggers, which require lepton $p_{T}>18 \mathrm{GeV} / c$ (see section 3.4). The distribution of lepton $p_{T}$ is shown in figure 3.4 for $t \bar{t}$ and $t \bar{t} H$, and demonstrates that we are cutting below the peak of the distribution. The $z_{0}$ cut is highly efficient and reduces the incidence of events which are outside the center of the detector and difficult to reconstruct correctly. We select events with exactly one lepton.

\subsection{Missing transverse energy}

Missing transverse energy, or $\mathbb{E}_{T}$, is an imbalance of energy deposited in the calorimeter. Because momentum is conserved, and the proton-antiproton system has very little transverse momentum, we expect that the vector sum of the transverse momenta of all outgoing particles will be zero. Since the masses of the final state particles are generally small compared to their energies, the calorimeter energy deposit is a reasonable approximation to the magnitude of the particle momenta, and the calorimeter segmentation provides an approximation to the direction as well. So, we expect that the vector sum of all the calorimeter energy deposits will also be very close to zero. When a large imbalance is observed, this is often an indication that a high- $p_{T}$ neutrino was produced in the event.

There are a number of ways in which a large imbalance in transverse energy can be 


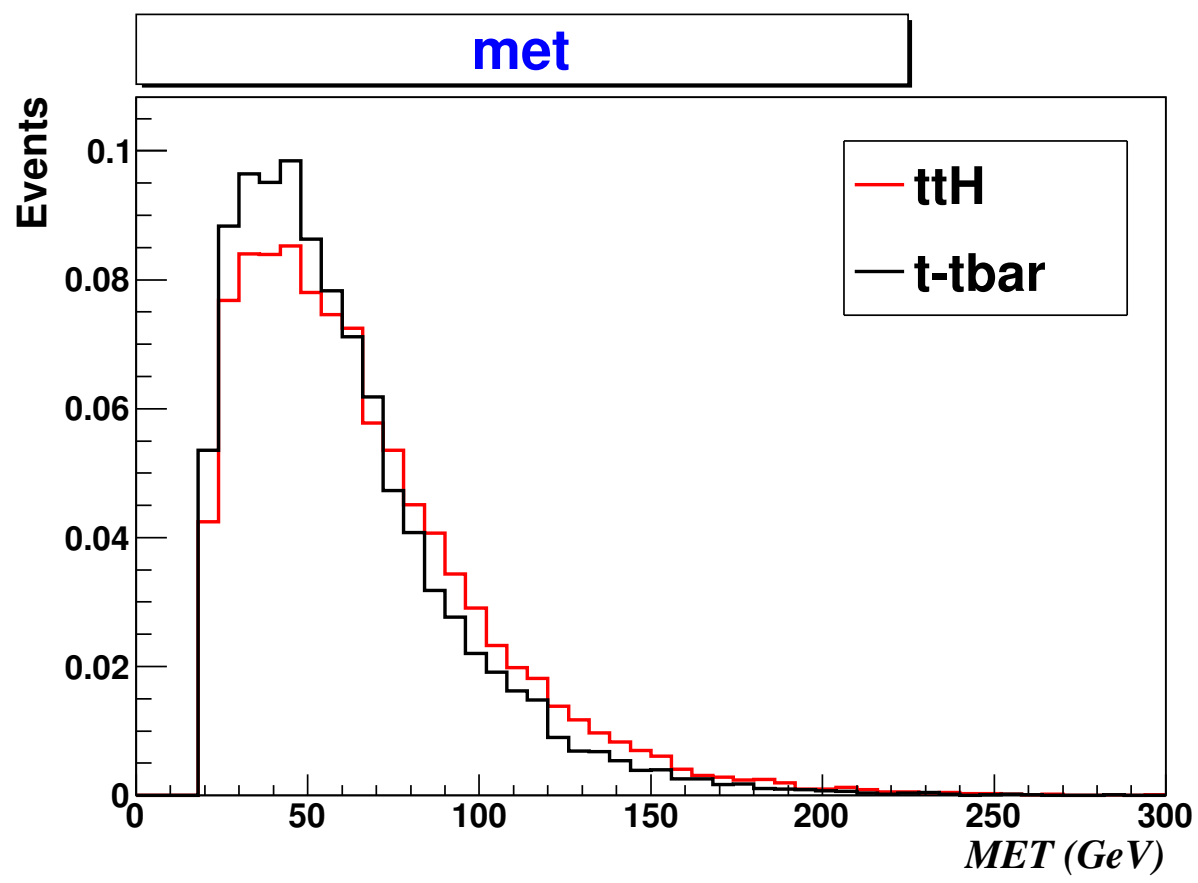

Figure 3.5: Missing transverse energy distribution in $t \bar{t}$ and $t \bar{t} H$

produced in the absence of a real neutrino. In order to select events with real neutrinos, we must attempt to reduce the number of events with fake $\mathbb{E}_{T}$ that enter our sample.

Muons can carry a significant amount of energy away from the collision, but, because they are minimum-ionizing particles, they do not leave that energy in the calorimeter. Thus, in an event with a real muon or muons but no neutrino, a significant $\mathbb{E}_{T}$ may be observed, but it will tend to point in the direction of the muon and have about the same magnitude as the momentum of the muon. By reconstructing even very low-quality muons and measuring their momenta, we can correct the $\mathbb{E}_{T}$ for these minimum-ionizing particles and reduce this source of background.

Hadrons are produced copiously at a proton-antiproton collider, as part of a jet, from the beam remnants, or from multiple interactions in a single bunch crossing. In order to accurately measure $\mathbb{E}_{T}$, it is crucial to account for all of the transverse energy that is carried by hadrons. The majority of the energy carried by hadrons is in jets, which are not always well-measured. After correcting the jet energy measurements (section 3.3), the $\mathbb{E}_{T}$ is also 


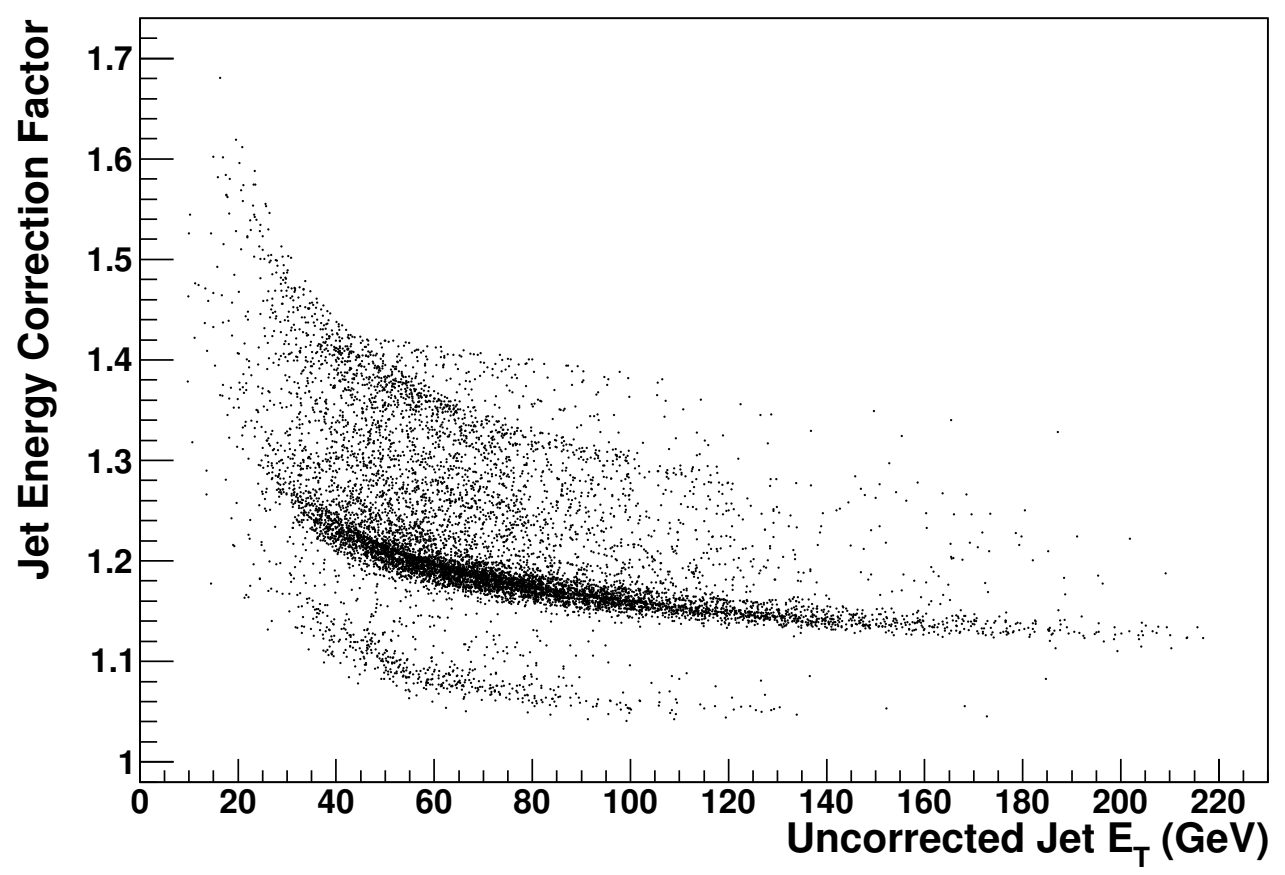

Figure 3.6: Jet energy correction factor as a function of uncorrected transverse energy

corrected accordingly[36].

We select events with at least $20 \mathrm{GeV}$ of corrected missing transverse energy $(25 \mathrm{GeV}$ for events with a PHX electron). This cut is below the kinematic peak in both the $t \bar{t}$ background and the $t \bar{t} H$ signal, as shown in figure 3.5. Observation of the signature of both a charged lepton and a neutrino is strongly indicative that a $W$ boson was produced in the event and decayed leptonically. Since the top quark decays to a $W$ boson and a $b$ quark, selecting events with a leptonic $W$ candidate removes an enormous amount of the multijet background while retaining nearly $20 \%$ (the branching ratio of $W$ to $e \nu$ or $\mu \nu$ ) of the $t \bar{t}$ and $t \bar{t} H$ events.

\subsection{Jets}

In addition to the leptonic $W$ boson, our signal events also contain two quarks from the decay of the second $W$ boson, two $b$ quarks from the top quark decays, and the Higgs decay products, which are principally either quarks from $H \rightarrow W W \rightarrow q q q q$ or $b$ quarks from $H \rightarrow b \bar{b}$. The strong interaction confines quarks to hadrons, and so the lone quarks produced 


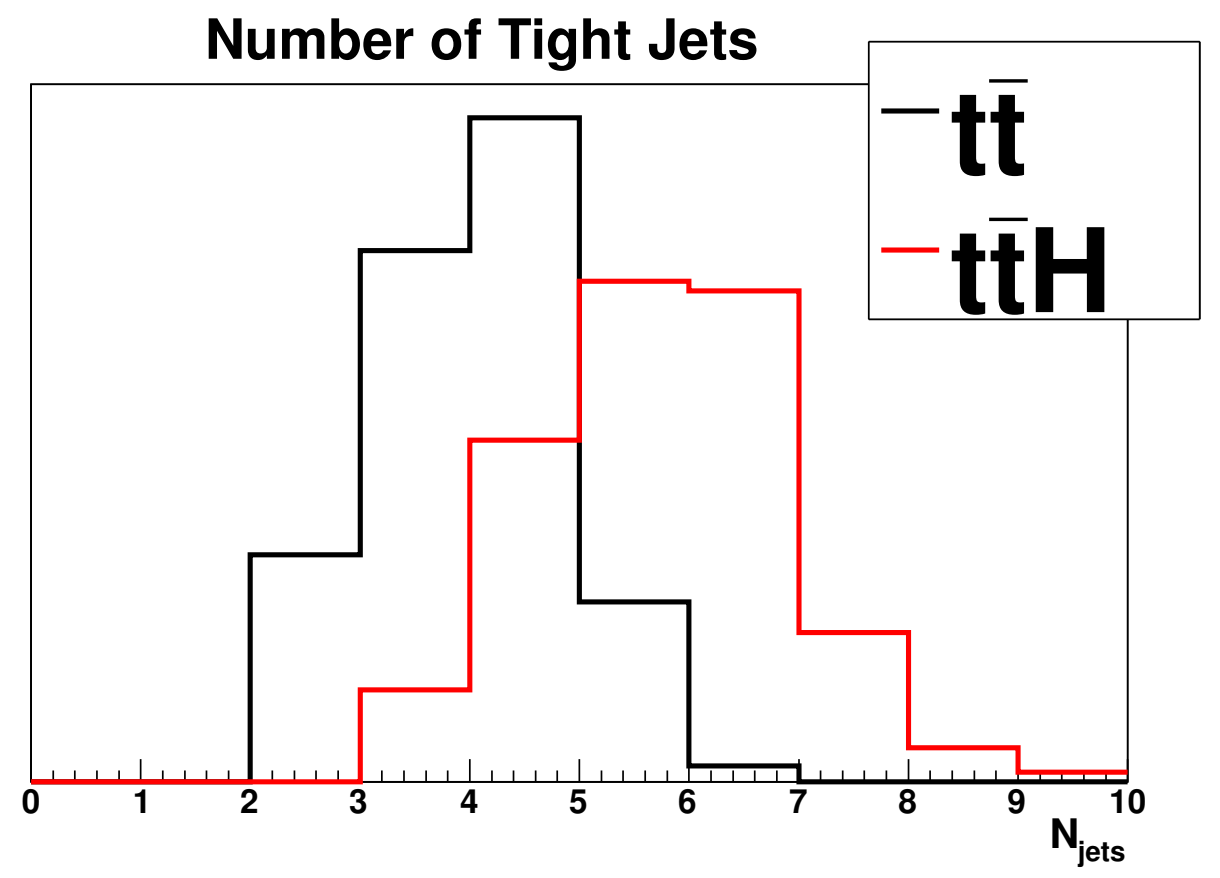

Figure 3.7: Distribution of the number of tight jets in $t \bar{t} H$ signal and $t \bar{t}$ background events

by these decays immediately hadronize by combining with quark-antiquark pairs from the vacuum until all the particles are in a color-neutral final state. This produces a broad spray of particles, mostly pions and kaons, centered around the direction of each quark. This spray of particles leaves a group of tracks and a cluster of energy in the calorimeter called a jet.

Jets are reconstructed in our detector using the JETCLU cone algorithm. This algorithm selects towers with at least $1 \mathrm{GeV}$ of energy as "seed" towers, and then draws a circle around them in $\eta-\phi$ space with a radius of 0.4 . A vector sum of the energies deposited in towers within that cone is formed, and the cone center is re-adjusted to the centroid of this sum. This process is iteratively repeated while combining cones with sufficient overlap until the remaining cones are stable against further iteration. The vector sum of the energies within each remaining cone is the reconstructed jet energy[37].

Because of a variety of factors, the measured energy in the jet cone is not identical to the outgoing parton momentum. In order to more accurately reconstruct the event, we attempt to account for each of these effects and correct the jet energies accordingly. 
Very low transverse momentum charged particles do not escape the solenoid. Their tracks describe a helix in the tracking chamber, and they never reach the calorimeter at all. While each of these particles does not carry a large percentage of the jet energy, the number of such particles may be large enough to noticeably affect the measurement. Particles with enough momentum to reach the calorimeter but with energy less than $10 \mathrm{GeV}$ are difficult to measure correctly, because the calorimeter exhibits a non-linear response to such low-energy particles. Additionally, low-momentum charged particles will have their trajectories bent sufficiently far by the solenoid field that they reach the calorimeter outside of the jet cone.

There are gaps in the active volume of the calorimeter. The calorimeter is segmented in both $\eta$ and $\phi$, and the edges of the individual modules making up the segmentation are not active. Thus, a hadron which showers in the area of such a gap will have a smaller fraction of its total energy recorded by the calorimeter.

At a hadron collider, there are a large number of additional particles produced in each interaction that are not truly part of the hard scatter process that we are attempting to reconstruct. This additional particle production is referred to as the underlying event. As the jet forms, some of the hadrons may be produced with a momentum that deviates sufficiently from the original parton direction that they do not fall within the jet cone. Some quarks, particularly $b$ and $c$ quarks, decay by emitting a $W$ boson while the jet is forming. This $W$ boson decays to a lepton and a neutrino $30 \%$ of the time. The neutrino momentum is not recorded by the calorimeter. If the lepton is a muon, then only a minimal energy deposit will be left in the calorimeter by the lepton. If the lepton is a $\tau$, it will decay by the emission of another $W$ boson, and there will be a second or possibly even a third neutrino in the jet.

These instrumental and physical factors affect the measured jet energy. We attempt to correct the jet energies by creating a correction function which is parameterized in $\eta_{\mathrm{d}}$ and jet $E_{T}$. This correction function is measured via a variety of techniques, including balancing against well-measured objects such as photons and leptonically-decaying $Z$ bosons, test-beam data, measurements of the momentum spectrum of charged particles in a jet, and others. As can be seen in figure 3.6, the correction factor is generally larger than 1 , indicating that we typicaly mismeasure jet energies too low as a result of the effects described above. The 


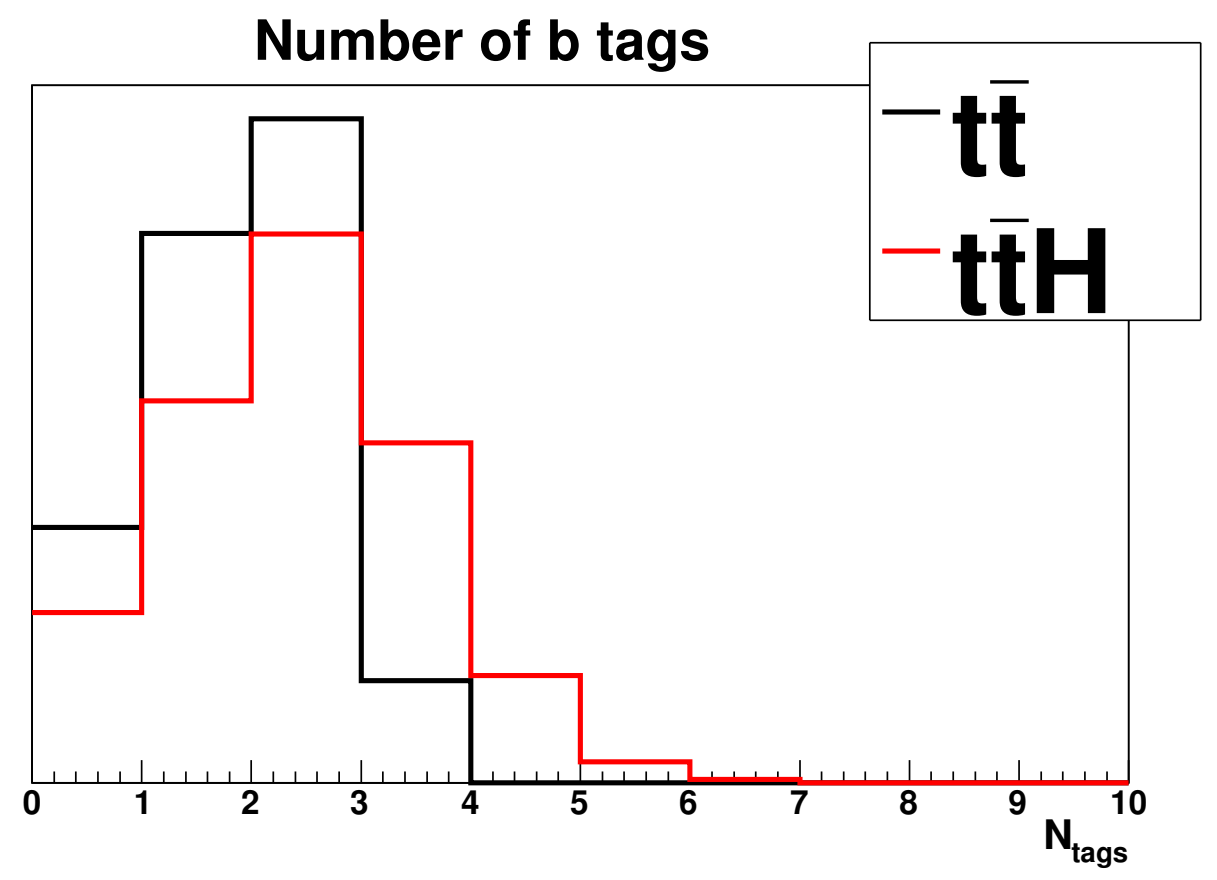

Figure 3.8: Distribution of the number of $b$-tagged jets in $t \bar{t} H$ signal and $t \bar{t}$ background events

residual uncertainty on the jet energies is treated as a systematic uncertainty[37].

After correcting the jet energies, we define "tight" jets to be jets with $E_{T}$ at least $20 \mathrm{GeV}$ and $\left|\eta_{\mathrm{d}}\right|<2.0$. We also define "loose" jets for the purpose of correcting the $\mathbb{E}_{T}$, with $E_{T}$ at least $12 \mathrm{GeV}$ and $\left|\eta_{\mathrm{d}}\right|<2.4$.

Since $t \bar{t} H$ has at least 4 quarks in the final state (in the case that the Higgs boson decays to zero final state quarks), we select events containing at least 4 tight jets. The 4 jet sample is overwhelmingly dominated by $t \bar{t}$ events, so we separate our selection into two subsets, one with exactly 4 jets and one with 5 or more jets (figure 3.7 ). The 4 jet region acts as a control region to effectively measure the rate of $t \bar{t}$ in situ, and the 5 jet region is our principal signal region.

\subsection{1 $b$ tagging}

Both of the quarks originating from top quark decay, as well as the Higgs decay products when the Higgs is at low mass, are $b$ quarks. In many cases, when a $b$ quark is produced, 
it first combines with another quark to form a $B$ meson. The $B$ mesons have a relatively long lifetime, on the order of $c \tau \approx 0.5 \mathrm{~mm}$. When the $b$ is energetic, as in the case of $b$ quarks produced from the decay of heavy particles like the top quark or the Higgs boson, this lifetime is increased by the gamma factor. For a typical jet with an energy of $\sim 50 \mathrm{GeV}$, the decay length for the $B$ meson is $\sim 5 \mathrm{~mm}$. After traveling some distance and decaying, the decay products hadronize and form a jet. The separation between the primary vertex where the $b$ quark and $B$ meson were produced and the location where the $B$ meson decays is large enough to be measurable with our high-precision silicon inner tracking system, and this measurement can be used to identify whether a jet originated from a $b$ quark or some other flavor of quark or gluon. This identification is called $b$-tagging.

There are two main $b$-tagging algorithms in use at CDF: SECVTX and Jet Probability. SECVTX directly reconstructs the displaced vertex associated with the $b$ jet, and then measures the distance between the primary and secondary vertices relative to the error ellipse associated with the reconstruction of each vertex. If the separation between the vertices is more than 3 sigma, and there are enough well-reconstructed tracks associated with each vertex, then the jet is $b$-tagged[38].

Jet Probability does not attempt to reconstruct a secondary vertex directly. Instead, it examines the impact parameter of each track associated with the jet relative to the location of the primary vertex. Based on the uncertainty on the reconstructed track parameters and the uncertainty on the reconstructed primary vertex position, a likelihood for each track to have originated at the primary vertex is formed. The likelihoods for all of the jet-associated tracks are combined to find the likelihood that the jet contains a displaced vertex. This gives a continuous variable which separates $b$ jets from light flavor jets. A number of different cuts on this likelihood can be used; CDF analyses in general and this analysis in particular use the $5 \%$ operating point, which gives good light flavor rejection and good $b$ tagging efficiency[36].

Because it is difficult to correctly simulate the very low-level properties of jets that are exploited by these tagging algorithms, the rate at which we tag $b$ jets, $c$ jets, and light flavor jets is slightly different in data than in Monte Carlo. In order to account for these 


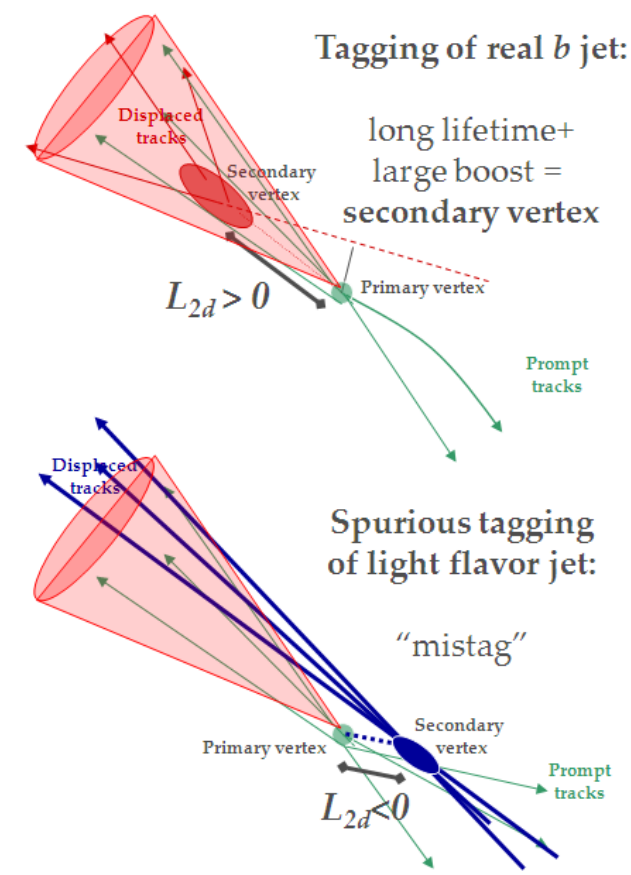

Figure 3.9: Positive and negative SECVTX tags

differences, we measure the relative rate of heavy flavor tagging in data and in Monte Carlo and use the ratio between the two as a scale factor to reduce the Monte Carlo heavy flavor tagging rate to match what is observed in data.

Because light flavor jets do not have true displaced vertices, the only way that light flavor jets are $b$ tagged is by chance. For instance, if a few tracks are slightly mismeasured, they could appear to originate from a secondary vertex. Naturally, this is a rare occurrence, but the number of light flavor jets is very large, so these "mistags" constitute a large fraction of the set of $b$ tagged jets. Unfortunately, any rare process that occurs only in the tail of distribution is difficult to model well, and Monte Carlo does not get the light flavor tagging rate quite right. In order to correctly account for the presence of light flavor $b$-tagged jets in our data, we use a data-driven method.

For SECVTX tags where a displaced vertex is fully reconstructed, we examine the direction from the primary vertex to the secondary vertex versus the direction of the jet momentum (figure 3.9). If these are no more than $90^{\circ}$ apart, then the tag is considered 


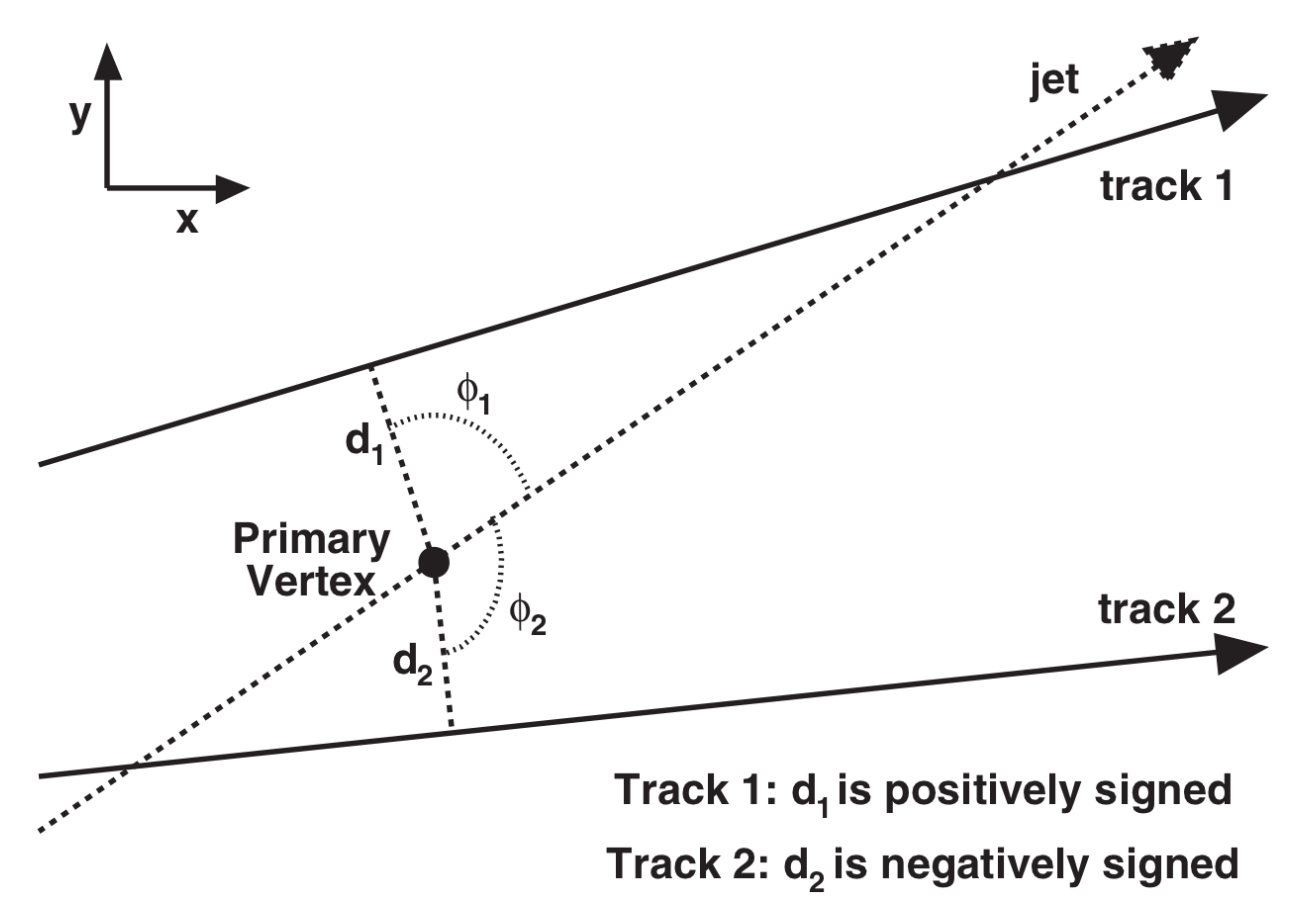

Figure 3.10: Positive and negative Jet Probability tracks

"positive" tag. Positive tags are the only variety of SECVTX tags that are actually used in the analysis as $b$ tags. In the case where the measured secondary vertex is not due to a real physical effect, we do not expect any correlation between the displacement direction and the jet direction. These mistags should occur at roughly the same rate for "negative" tags where the displacement direction and jet direction are more than $90^{\circ}$ apart as they do for positive tags. The negative tag rate, after some additional corrections for second-order effects, is used to estimate the mistag rate[38]. For Jet Probability tags, a similar technique is used to measure the mistag rate (figure 3.10)[36]. The mistag rates as measured in data using negative tags are used to populate a "mistag matrix" for each tagging algorithm, which parameterizes the light flavor tagging rate as a function of the gross properties (such as $E_{T}$ and $\eta_{\mathrm{d}}$ ) of the jet, which are well-modeled by our Monte Carlo.

In Monte Carlo, jets that originated from a $b$ or a $c$ quark are identified by examining the list of particles generated by the Monte Carlo hard scatter process. For those jets that match a heavy flavor quark, we apply the tagging algorithms to the jet. If the jet is tagged 
by the tagging algorithm, then we generate a random number. If that number is less than the scale factor (0.96 for SECVTX and 0.78 for Jet Probability), then we consider that jet to have been tagged by that algorithm. Otherwise, we ignore the tag. This is sufficient to account for the differences in the tagging rate for true heavy flavor jets between Monte Carlo and data.

If a Monte Carlo jet is matched to a light flavor quark, a gluon, or no colored object at all, then we do not apply the tagging algorithm. Instead, we evaluate the mistag matrix for that jet, and again generate a random number to determine whether or not we consider that jet to be tagged. In an analysis with high jet multiplicity and a complex tagging structure, this random number method greatly simplifies the use of tagging, as compared to other analyses that use the scale factor and mistag matrix probability as weights when filling histograms.

The mistag rejection rate for SECVTX is better than for Jet Probability, but the SECVTX $b$ tagging rate is smaller. Because of this, we always attempt to tag jets using SECVTX first, and only if they are not tagged by SECVTX do we consider Jet Probability tags. This allows us to develop samples of multiply-tagged events with varying purities of $b$ jets versus mistags.

Because of the high expected $b$ jet multiplicity in our signal (figure 3.8), we require at least $2 b$-tagged jets. In order to isolate a sample containing less $t \bar{t}$ and more $t \bar{t} H$ signal, we further divide this into a 2 tag and a 3 tag sample, each of which is further divided according to the number of the tags that were provided by each of the tagging algorithms. We use five tagging categories:

STST 2 SECVTX tags,

STJP 1 SECVTX tag and 1 Jet Probability tag,

STSTST 3 or more SECVTX tags,

STSTJP 2 SECVTX tags and 1 or more Jet Probability tags, and

STJPJP 1 SECVTX tag and 2 or more Jet Probability tags. 

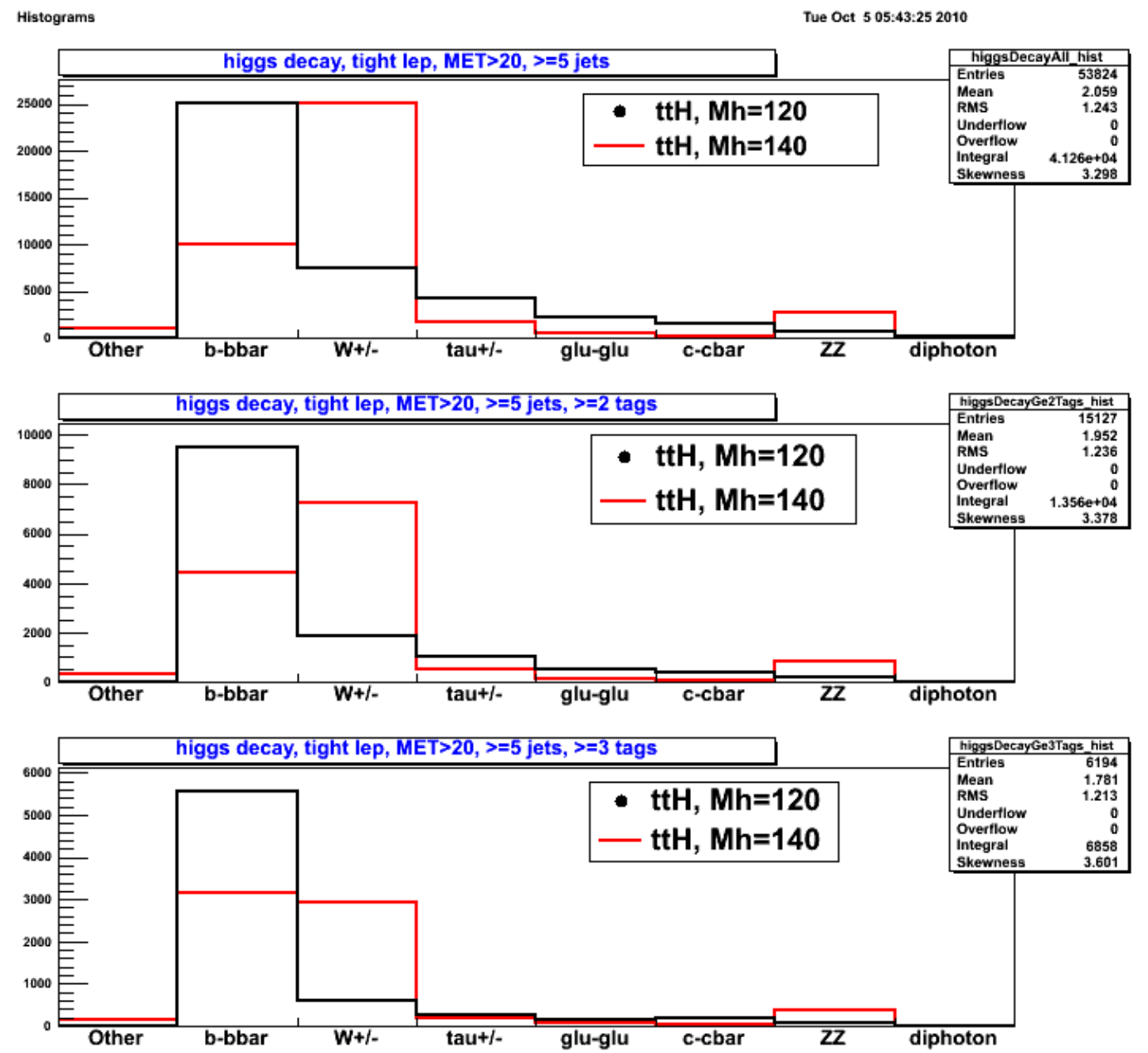

Figure 3.11: Contribution of different Higgs decays to our untagged, 2 tag, and $\geq 3$ tag samples 
In the high tag multiplicity sample, not all of our tags come from Higgs decay $b$ quarks (figure 3.11). The $W$ boson decays some of the time to $c \bar{s}$, and the charm quark originated jet is often $b$-tagged as well. In events where the Higgs boson decays to a $W$ pair, the chance of tagging a charm jet from $W$ decay is substantially increased, and so these events appear in our data sample at a substantial rate. The Higgs also decays directly to a pair of charm quarks, or to a pair of $\tau$ leptons, which also often produce $b$-tagged jets when they decay hadronically.

\subsection{Triggers}

In order for an event to enter our sample, it must first be accepted by our trigger system (section 2.5). We use the high- $P_{T}$ lepton triggers for this analysis. CEM electrons are selected by the ELECTRON_CENTRAL_18 trigger. PHX electrons are selected by the MET_PEM trigger. CMUP muons are selected by the MUON_CMUP18 trigger and CMX muons by the MUON_CMX18 trigger.

All of these triggers exist in multiple versions. Over the entirety of Tevatron Run II, there were many changes to the trigger tables necessitated by the increasing luminosity profile of the physics stores, the changing physics priorities of the collaboration, changes

in the detector performance, and trigger hardware upgrades. Even without the necessary alterations to each trigger, the tape system has a limited bandwidth, and many basic triggers like those listed above have a rate that, at very high luminosities, would overwhelm our ability to write events to tape. In order to keep trigger rates to a manageable level, some versions of the trigger used a luminosity enable, in which the trigger would be completely turned off if the instantaneous luminosity was above a threshold. Other versions used a prescale, in which only a fraction of the events for which the trigger fired would be written to tape. Some versions used a dynamic prescale, changing the accepted fraction of fired events as the instantaneous luminosity changed. At very high luminosity, in order to retain as many interesting events as possible, versions of the triggers with a less aggressive prescale were implemented with an additional requirement, such as a jet with a transverse energy 


\begin{tabular}{rrr}
\hline Channel & $N_{\text {data }}(4$ jets $)$ & $N_{\text {data }}(5+$ jets $)$ \\
\hline STSTST & 14 & 11 \\
STSTJP & 34 & 20 \\
STJPJP & 25 & 17 \\
STST & 258 & 112 \\
STJP & 303 & 98 \\
\hline
\end{tabular}

Table 3.3: Number of data events in each analysis channel

of $10 \mathrm{GeV}$. During data period 18 , the muon triggers were not functioning properly in some runs. Muon events in these runs have been recovered using the loose muon trigger MUON_CMUP18_LOOSE_DPS.

These triggers all require that the identified lepton have a transverse momentum of at least $18 \mathrm{GeV} / c$. However, especially for events with leptons that are very close to that threshold, the trigger does not accept $100 \%$ of the events that we might expect it should. In order to avoid the complexity of evaluating the rate at which the trigger accepts events, we only accept events in which the identified lepton has at least $20 \mathrm{GeV} c^{-1}$ of transverse momentum. Above this cut, the trigger efficiency is more or less constant, and we can simply measure that constant rate, which ranges from $85 \%$ to $99 \%$ depending on the lepton type.

\subsection{Summary}

We select a sample of events with:

- exactly one tight electron or muon with at least $20 \mathrm{GeV}$ of transverse momentum from the ELECTRON_CENTRAL_18, MET_PEM, MUON_CMUP18, or MUON_CMX18 triggers,

- at least $20 \mathrm{GeV}$ of $\mathbb{E}_{T}$, after correcting for muons and jet energy corrections,

- at least 4 tight jets with transverse energy at least $20 \mathrm{GeV}$ and in the central detector $\left(\left|\eta_{\mathrm{d}}\right|<2.0\right)$

- at least $2 b$-tagged jets using the SECVTX and Jet Probability algorithms 
This sample is further divided up by the number of jets (4 jets versus 5 or more jets) and the number and type of tags (two 2-tag and three 3-tag channels), yielding a total of 10 analysis channels. The number of data events observed in each channel is shown in table 3.3. 


\section{Chapter 4 \\ Data Modeling}

In order to determine whether or not events containing a Higgs boson are present in our data sample, we must first identify what other processes are involved in producing the observed data. These other processes are our "backgrounds", and the hypothetical events involving the Higgs boson are our "signal".

\subsection{Monte Carlo simulation}

Because we believe that the Standard Model is an accurate, albeit likely incomplete, description of the universe, we should be able to use it to make predictions regarding the contents and distribution of our data. In principle, one would like to calculate the full Standard Model $S$ matrix to arbitrary order, and then integrate over the available phase space to determine the distribution of any observable in our data. Because this is computationally utterly infeasible, we resort to numerical methods to determine these observable distributions. Instead of analytic integration, we perform a Monte Carlo integration using programs such as PYTHIA, MadGraph, and ALPGEN[39, 40, 41].

These programs pseudorandomly generate simulated hard scattering events one at a time. The parameters of each event are distributed according to the predictions of the Standard Model up to leading order for a given process. With a large enough set of simulated events, the leading order predictions for the distribution of any hard scatter level variable can be evaluated with arbitrary precision.

The outgoing colored particles from these events are then passed to PYTHIA to be 
showered into particle jets. The shower is accomplished via a Monte Carlo sampling using the Lund string model, which models all but the very highest energy gluons as "strings", or long, narrow regions of color flux. New quarks are generated by randomly "breaking" the gluon strings by creating a $q \bar{q}$ pair. These new quarks then combine with other quarks to produce colorless hadrons. This process is governed by a large number of parameters, including a shower cutoff scale and the strong coupling constant. PYTHIA has been semiempirically tuned to reproduce the observed jet fragmentation properties at CDF, such as the $p_{T}$ spectrum of the hadrons in a jet, as well as the underlying event[39].

The final state particles from PYTHIA are then passed to a full GEANT simulation of the CDF detector. GEANT simulates the interaction of high energy particles with matter and produces the simulated electrical signals that we would expect to read from the detector[42].

After completely simulating the entire event, hadron shower, and detector response, we use the normal CDF reconstruction software to produce a full simulation of what we expect our data to look like for each background process. A large enough sample of such fullysimulated Monte Carlo events, once all relevant processes are generated, should accurately reproduce our data in all distributions.

This procedure does a very good job of modeling the data, but some discrepancies still remain. It is computationally difficult to model any rare process, such as events occurring in the tail of a distribution, by Monte Carlo integration. These processes can be modeled using data taken from a control region that is orthogonal to the signal region. Additionally, small discrepancies in fine details such as the track multiplicity, distribution of track impact parameters, calorimeter shower profiles, etc. are taken into account by measuring scale factors in order to downweight some Monte Carlo events to better match the data. In order to model the busy environment of a high luminosity hadron collider, we take "min-bias" data, which is a small sample of all bunch crossings involving any detector activity, and overlay it onto our Monte Carlo events. Finally, we correct for the largest differences between using leading-order versus next-to-leading-order calculations in our Monte Carlo generators by applying " $k$-factors" to some Monte Carlo samples. 


\begin{tabular}{lrr}
\hline Sample & \multicolumn{1}{c}{$\boldsymbol{N}_{\text {jets }}=\mathbf{4}$} & \multicolumn{1}{c}{$\boldsymbol{N}_{\text {jets }} \geq \mathbf{5}$} \\
\hline DiTop & $16.89 \pm 3.97$ & $8.63 \pm 1.89$ \\
STopT & $0.07 \pm 0.01$ & $0.02 \pm 0.01$ \\
STopS & $0.11 \pm 0.02$ & $0.05 \pm 0.01$ \\
Wbb & $0.74 \pm 0.30$ & $0.37 \pm 0.15$ \\
Wcc & $0.05 \pm 0.02$ & $0.04 \pm 0.02$ \\
Wcj & $0.02 \pm 0.01$ & $0.02 \pm 0.01$ \\
Zjets & $0.02 \pm 0.00$ & $0.01 \pm 0.00$ \\
WW & $0.06 \pm 0.01$ & $0.00 \pm 0.00$ \\
WZ & $0.02 \pm 0.00$ & $0.01 \pm 0.00$ \\
ZZ & $0.00 \pm 0.00$ & $0.00 \pm 0.00$ \\
Non-W & $0.00 \pm 0.00$ & $0.00 \pm 0.00$ \\
Mistags & $0.02 \pm 0.01$ & $0.01 \pm 0.01$ \\
\hline Total Prediction & $18.00 \pm 3.99$ & $9.16 \pm 1.90$ \\
\hline ttH120 & $0.06 \pm 0.01$ & $0.31 \pm 0.03$ \\
\hline Observed & 14 & 11 \\
\hline
\end{tabular}

Table 4.1: Background from various sources compared to observed data $(\mathrm{CEM}+\mathrm{PHX}+\mathrm{CMX}+\mathrm{CMUP})$, for the STSTST tagging category.

\subsection{Backgrounds}

Backgrounds that have all of the correct final state objects to naturally fall into our sample are called "irreducible" backgrounds. Those that lack the final state we select for but fake the selected signature are called "reducible" backgrounds. The predicted contribution of each background to our data sample is given in tables 4.1-4.5, where the uncertainties are the combined effects of the systematic uncertainties described in chapter 6 .

\subsubsection{Irreducible}

In a sample of events with a lepton, $\mathbb{E}_{T}$, a high jet multiplicity, and a high $b$-tag multiplicity, by far the predominant contributing process is top quark pair production in the lepton+jets channel. We expect this process to produce more than $85 \%$ of our data sample. In the three-tag channel, the third tag can come from a charm quark in $W \rightarrow c s$ or from an initialor final-state radiation gluon that splits to $b \bar{b}$. The $t \bar{t}$ process is modeled using PYTHIA 


\begin{tabular}{lrr}
\hline Sample & \multicolumn{1}{c}{$\boldsymbol{N}_{\text {jets }}=\mathbf{4}$} & \multicolumn{1}{c}{$\boldsymbol{N}_{\text {jets }} \geq \mathbf{5}$} \\
\hline DiTop & $39.61 \pm 8.40$ & $18.86 \pm 3.98$ \\
STopT & $0.19 \pm 0.04$ & $0.07 \pm 0.01$ \\
STopS & $0.28 \pm 0.05$ & $0.09 \pm 0.02$ \\
Wbb & $1.77 \pm 0.77$ & $0.79 \pm 0.34$ \\
Wcc & $0.27 \pm 0.12$ & $0.14 \pm 0.07$ \\
Wcj & $0.13 \pm 0.06$ & $0.05 \pm 0.03$ \\
Zjets & $0.06 \pm 0.01$ & $0.03 \pm 0.01$ \\
WW & $0.06 \pm 0.01$ & $0.02 \pm 0.01$ \\
WZ & $0.03 \pm 0.01$ & $0.01 \pm 0.00$ \\
ZZ & $0.00 \pm 0.00$ & $0.00 \pm 0.00$ \\
Non-W & $0.53 \pm 2.00$ & $0.36 \pm 2.00$ \\
Mistags & $0.11 \pm 0.05$ & $0.06 \pm 0.03$ \\
\hline Total Prediction & $43.04 \pm 8.67$ & $20.48 \pm 4.47$ \\
\hline ttH120 & $0.06 \pm 0.01$ & $0.26 \pm 0.02$ \\
\hline Observed & 34 & 20 \\
\hline
\end{tabular}

Table 4.2: Background from various sources compared to observed data $(\mathrm{CEM}+\mathrm{PHX}+\mathrm{CMX}+\mathrm{CMUP})$, for the STSTJP tagging category.

\begin{tabular}{lrr}
\hline Sample & \multicolumn{1}{c}{$\boldsymbol{N}_{\text {jets }}=\mathbf{4}$} & \multicolumn{1}{c}{$\boldsymbol{N}_{\text {jets }} \geq \mathbf{5}$} \\
\hline DiTop & $23.52 \pm 3.82$ & $11.86 \pm 2.15$ \\
STopT & $0.12 \pm 0.02$ & $0.04 \pm 0.01$ \\
STopS & $0.16 \pm 0.03$ & $0.05 \pm 0.01$ \\
Wbb & $1.25 \pm 0.54$ & $0.56 \pm 0.25$ \\
Wcc & $0.42 \pm 0.19$ & $0.22 \pm 0.10$ \\
Wcj & $0.21 \pm 0.10$ & $0.09 \pm 0.04$ \\
Zjets & $0.06 \pm 0.01$ & $0.03 \pm 0.01$ \\
WW & $0.05 \pm 0.02$ & $0.03 \pm 0.01$ \\
WZ & $0.03 \pm 0.01$ & $0.01 \pm 0.00$ \\
ZZ & $0.00 \pm 0.00$ & $0.00 \pm 0.00$ \\
Non-W & $0.36 \pm 2.00$ & $0.55 \pm 1.94$ \\
Mistags & $0.23 \pm 0.12$ & $0.11 \pm 0.06$ \\
\hline Total Prediction & $26.41 \pm 4.36$ & $13.55 \pm 2.91$ \\
\hline ttH120 & $0.02 \pm 0.00$ & $0.11 \pm 0.01$ \\
\hline Observed & 25 & 17 \\
\hline
\end{tabular}

Table 4.3: Background from various sources compared to observed data $(\mathrm{CEM}+\mathrm{PHX}+\mathrm{CMX}+\mathrm{CMUP})$, for the STJPJP tagging category. 


\begin{tabular}{lrr}
\hline Sample & \multicolumn{1}{c}{$\boldsymbol{N}_{\text {jets }}=\mathbf{4}$} & \multicolumn{1}{c}{$\boldsymbol{N}_{\text {jets }} \geq \mathbf{5}$} \\
\hline DiTop & $236.96 \pm 23.35$ & $84.13 \pm 8.42$ \\
STopT & $2.24 \pm 0.22$ & $0.44 \pm 0.04$ \\
STopS & $2.09 \pm 0.17$ & $0.44 \pm 0.04$ \\
Wbb & $18.08 \pm 7.76$ & $5.19 \pm 2.31$ \\
Wcc & $7.55 \pm 3.31$ & $2.54 \pm 1.16$ \\
Wcj & $3.76 \pm 1.64$ & $1.03 \pm 0.47$ \\
Zjets & $1.39 \pm 0.19$ & $0.42 \pm 0.06$ \\
WW & $1.14 \pm 0.25$ & $0.40 \pm 0.09$ \\
WZ & $0.41 \pm 0.06$ & $0.13 \pm 0.02$ \\
ZZ & $0.04 \pm 0.00$ & $0.01 \pm 0.00$ \\
Non-W & $11.92 \pm 10.03$ & $3.29 \pm 3.49$ \\
Mistags & $6.81 \pm 2.36$ & $2.15 \pm 0.93$ \\
\hline Total Prediction & $292.39 \pm 26.93$ & $100.17 \pm 9.53$ \\
\hline ttH120 & $0.13 \pm 0.01$ & $0.36 \pm 0.02$ \\
\hline Observed & 303 & 98 \\
\hline
\end{tabular}

Table 4.4: Background from various sources compared to observed data $(\mathrm{CEM}+\mathrm{PHX}+\mathrm{CMX}+\mathrm{CMUP})$, for the STJP tagging category.

\begin{tabular}{lcc}
\hline Sample & $\boldsymbol{N}_{\text {jets }}=\mathbf{4}$ & \multicolumn{1}{c}{$\boldsymbol{N}_{\text {jets }} \geq \mathbf{5}$} \\
\hline DiTop & $256.86 \pm 33.06$ & $84.10 \pm 10.15$ \\
STopT & $2.59 \pm 0.31$ & $0.51 \pm 0.06$ \\
STopS & $2.26 \pm 0.24$ & $0.46 \pm 0.05$ \\
Wbb & $15.72 \pm 6.76$ & $4.16 \pm 1.85$ \\
Wcc & $2.37 \pm 1.03$ & $0.83 \pm 0.38$ \\
Wcj & $1.18 \pm 0.51$ & $0.34 \pm 0.15$ \\
Zjets & $0.86 \pm 0.11$ & $0.28 \pm 0.04$ \\
WW & $0.50 \pm 0.09$ & $0.15 \pm 0.03$ \\
WZ & $0.30 \pm 0.04$ & $0.08 \pm 0.01$ \\
ZZ & $0.06 \pm 0.01$ & $0.01 \pm 0.00$ \\
Non-W & $5.95 \pm 5.76$ & $2.58 \pm 3.06$ \\
Mistags & $1.68 \pm 0.54$ & $0.56 \pm 0.23$ \\
\hline Total Prediction & $290.33 \pm 34.26$ & $94.06 \pm 10.77$ \\
\hline ttH120 & $0.14 \pm 0.01$ & $0.38 \pm 0.03$ \\
\hline Observed & 258 & 112 \\
\hline
\end{tabular}

Table 4.5: Background from various sources compared to observed data $(\mathrm{CEM}+\mathrm{PHX}+\mathrm{CMX}+\mathrm{CMUP})$, for the STST tagging category. 


\section{Monte Carlo.}

The second largest background is $W$ plus jets, especially $W+b \bar{b}$. This is among the most complex processes to model using Monte Carlo. The distribution of the number of additional partons produced in the hard scatter with the $W$ falls very steeply, especially when some of them are heavy-flavored partons, so we model this background using a large number of separate Monte Carlo samples generated by ALPGEN and showered with PYTHIA. First, we divide the sample generation process by the decay of the $W$ (to $e, \mu$, or $\tau$ plus a neutrino). Then, we generate separate samples with $0,1,2,3$, and 4 or more hard scatter light-flavor partons, samples with two $b$ quarks or two $c$ quarks and 0,1 , and 2 or more additional light-flavor partons, and samples with one $c$ quark and $0,1,2$, or 3 or more additional light-flavor partons. This ensures that we have sufficient numbers of generated Monte Carlo events even far out in the tails of the additional-partons distribution. To recombine these samples into the full $W$ plus jets prediction, we scale each one by the predicted cross section and add them together[41].

The remaining irreducible backgrounds are single top production, in which a $W$ boson decays to a top-bottom pair ( $s$-channel) or converts an initial state $b$ into a $t(t$-channel), and diboson production ( $W W, W Z$, and $Z Z$ ). The single top background is modeled using MadGraph Monte Carlo with PYTHIA showering, and the diboson background is modeled using PYTHIA. These backgrounds contribute only a very small amount to our data sample.

\subsubsection{Reducible}

The largest reducible background is "Non- $W$ ", in which only quantum chromodynamic processes occur, producing only jets in the final state, but in which one of the jets fakes a lepton and the event is mismeasured in such a way as to generate fake $\mathbb{E}_{T}$. QCD multijet production that fakes the signature of a $W$ is exceedingly rare, especially in events that have 4 or more jets (at least two of which are $b$-tagged) in addition to the fake $W$. However, the total multijet cross section at a hadron collider is enormous, and so this rare occurrence is a significant contributor to our data sample. This is a larger contributor to the electron sample than to the muon sample, and is the third largest background overall. 
Because the Non- $W$ background is produced only via the extreme tail of the QCD multijet process, it is nearly impossible to model accurately using Monte Carlo. In order to generate a substantial sample of QCD multijet events that pass our event selection, we would need to generate a technically infeasible number of Monte Carlo events. As a result, we use a data-driven model for this background. We select a sample of events from our data that have an electron or muon candidate that fails any pair of the criteria given in section 3.1 but all the rest. This gives us a sample of data events that consists mostly of fake leptons, and which is entirely non-overlapping with our signal sample. We rescale this sample according to the fit described in section 4.4 and use it as our Non- $W$ model.

For the $Z$ plus jets sample, we use a set of ALPGEN Monte Carlo showered with PYTHIA that is generated according to the same scheme as is used for the $W$ plus jets background. Because events containing a $Z$ boson have either two or zero real leptons, these events only appear in our sample if one of the leptons is lost or some other object is mis-reconstructed as a lepton. Leptons can be lost through geometric acceptance effects (lepton travels down the beamline or hits a gap in detector coverage) or by overlapping with a jet. Fake leptons appear in the same ways as with the Non- $W$ background. Neither possibility is very likely, and so the $Z$ plus jets background is very small.

\subsection{Signal}

We model the $t \bar{t} H$ signal using PYTHIA Monte Carlo at 12 mass points: $100-150 \mathrm{GeV} / c^{2}$ in steps of $5 \mathrm{GeV} / c^{2}$, and a single high-mass sample at $170 \mathrm{GeV} / c^{2}$. These samples are generated using the Standard Model predicted couplings of the Higgs. The decay of the Higgs is not forced, but all of the Higgs decays are included with the predicted branching ratios at each mass. 


\begin{tabular}{cc}
\hline $\boldsymbol{m}_{\boldsymbol{H}}\left(\mathrm{GeV} / c^{2}\right)$ & $\boldsymbol{\sigma}(\mathrm{fb})$ \\
\hline 100 & 8.000 \\
105 & 7.062 \\
110 & 6.233 \\
115 & 5.502 \\
120 & 4.857 \\
125 & 4.279 \\
130 & 3.769 \\
135 & 3.320 \\
140 & 2.925 \\
145 & 2.593 \\
150 & 2.298 \\
170 & 1.430 \\
\hline
\end{tabular}

Table 4.6: Predicted $t \bar{t} H$ production cross section[1, 2]

\begin{tabular}{ccc}
\hline $\boldsymbol{m}_{\boldsymbol{H}}\left(\mathrm{GeV} / c^{2}\right)$ & $\boldsymbol{N}_{\text {jets }}=\mathbf{4}$ & $\boldsymbol{N}_{\text {jets }} \geq \mathbf{5}$ \\
\hline 100 & 0.750 & 2.19 \\
105 & 0.653 & 1.97 \\
110 & 0.552 & 1.72 \\
115 & 0.487 & 1.57 \\
120 & 0.405 & 1.38 \\
125 & 0.344 & 1.24 \\
130 & 0.283 & 1.09 \\
135 & 0.225 & 0.938 \\
140 & 0.191 & 0.852 \\
145 & 0.151 & 0.745 \\
150 & 0.126 & 0.688 \\
170 & 0.056 & 0.432 \\
\hline
\end{tabular}

Table 4.7: Expected number of selected $t \bar{t} H$ events with at least two $b$ tags in $7.5 \mathrm{fb}^{-1}$ 


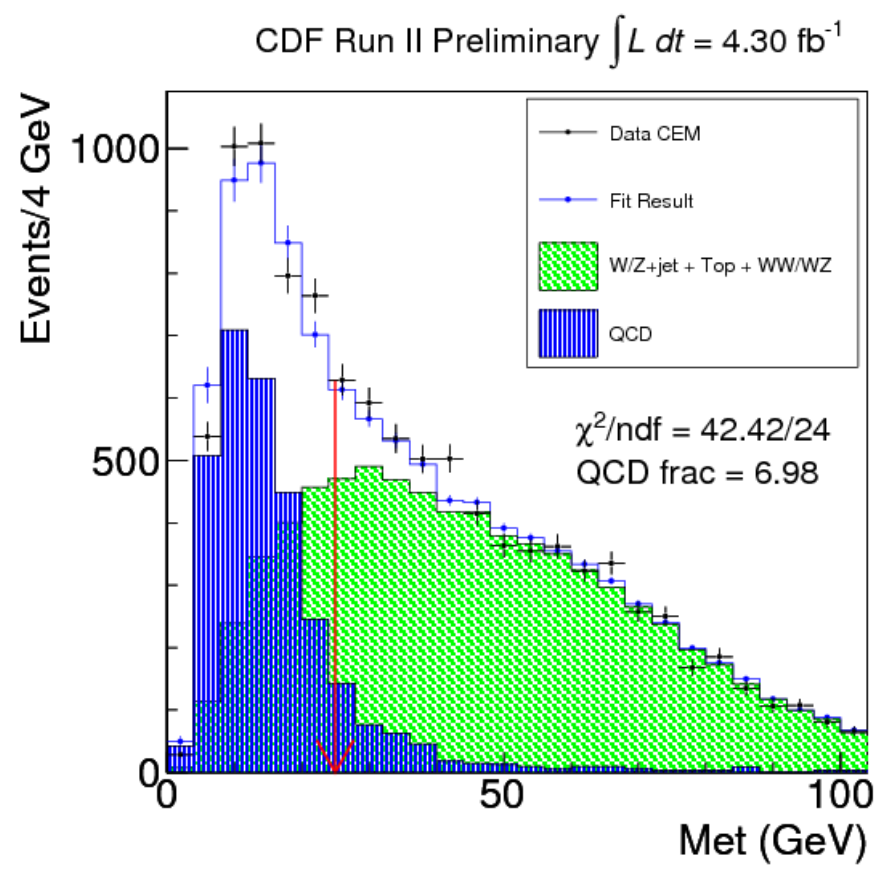

Figure 4.1: Fit to $\mathbb{E}_{T}$ distribution

\subsection{Estimating the background rates}

In order to estimate the rate of the various backgrounds, the simplest procedure is to use the predicted cross section and branching ratio and the total integrated luminosity:

$$
N_{p \bar{p} \rightarrow X}=\sigma_{p \bar{p} \rightarrow X} \cdot \int \mathcal{L} d t \cdot \epsilon_{\mathrm{MC}}
$$

where $N_{p \bar{p} \rightarrow X}$ is the total predicted number of events for a process in our sample, $\sigma_{p \bar{p} \rightarrow X}$ is the predicted cross section times branching ratio for that process, $\int \mathcal{L} d t$ is the total integrated luminosity used in our analysis, and $\epsilon_{\mathrm{MC}}$ is the selection efficiency for the process, estimated by the ratio of the number of selected Monte Carlo events to the number of generated Monte Carlo events, $N_{\text {selected }} / N_{\text {generated }}$. Because we have no reason to expect the Monte Carlo to model the potential $t \bar{t} H$ signal poorly, we use this method for the signal model.

This procedure also suffices for the $t \bar{t}$, single top, diboson, and $Z$ plus jets backgrounds (collectively referred to as electroweak backgrounds), which are modeled quite well by the 
Monte Carlo. For the $W$ plus jets and Non- $W$ backgrounds, a more sophisticated procedure is required. We need to make certain that we get the heavy flavor fraction and tagging rate on both heavy and light flavor jets correct in $W$ plus jets. The data-driven method we use to estimate our Non- $W$ background does not provide a predicted cross section nor a predicted selection efficiency, so we derive the Non- $W$ rate entirely from a sideband fit.

In order to obtain the fraction of our pretag sample which is composed of Non- $W$ events, we use the pretag $\mathbb{E}_{T}$ distribution (before the $\mathbb{E}_{T}$ cut in our event selection) of the Non- $W$ model events (anti-leptons) and of the $W$ plus jets Monte Carlo events. These two distributions are fit to the pretag $\mathbb{E}_{T}$ distribution in data, and the best-fit rate for the Non- $W$ template is used as the pretag Non- $W$ fraction (figure 4.1).

Since the multiply-tagged sample is our signal region, it is most important to properly estimate the tagging rate of each of our backgrounds. Once again, the Monte Carlo models the $t \bar{t}$, single top, diboson, and $Z$ plus jets backgrounds adequately, after we account for differences between data and Monte Carlo tagging via the scale factor and mistag matrix discussed above. The heavy flavor fraction in $W$ plus jets and the tagging rate in Non- $W$ need to be handled carefully in order to model the data well. The fitting procedure in $\mathbb{E}_{T}$ is repeated in the tagged data sample, using the full Monte Carlo background estimate instead of the $W$ plus jets only template used in the pretag fit. The pretag Non- $W$ fraction is used in estimating the tagged $W$ plus light flavor and $W$ plus heavy flavor background rates, and the tagged Non- $W$ fraction is simply used in modeling the tagged Non- $W$ background.

In order to estimate the rate of $W$ plus heavy flavor, the heavy flavor fraction in $W$ plus jets events is estimated from the ALPGEN Monte Carlo and multiplied by a $k$-factor that accounts for the differences between leading-order and next-to-leading-order calculations of the heavy flavor fraction. The heavy flavor fraction and $k$-factor are then multiplied by the number of pretag events in data that are not expected to be from the Non- $W$ or electroweak backgrounds to obtain the predicted number of tagged $W$ plus heavy flavor events.

In order to estimate the rate of $W$ plus light flavor, the mistag matrix is used to predict mistag rates for pretag data, $W$ plus heavy flavor, Non- $W$, and the electroweak backgrounds. The total predicted mistag rate for the backgrounds is subtracted from the predicted mistag 


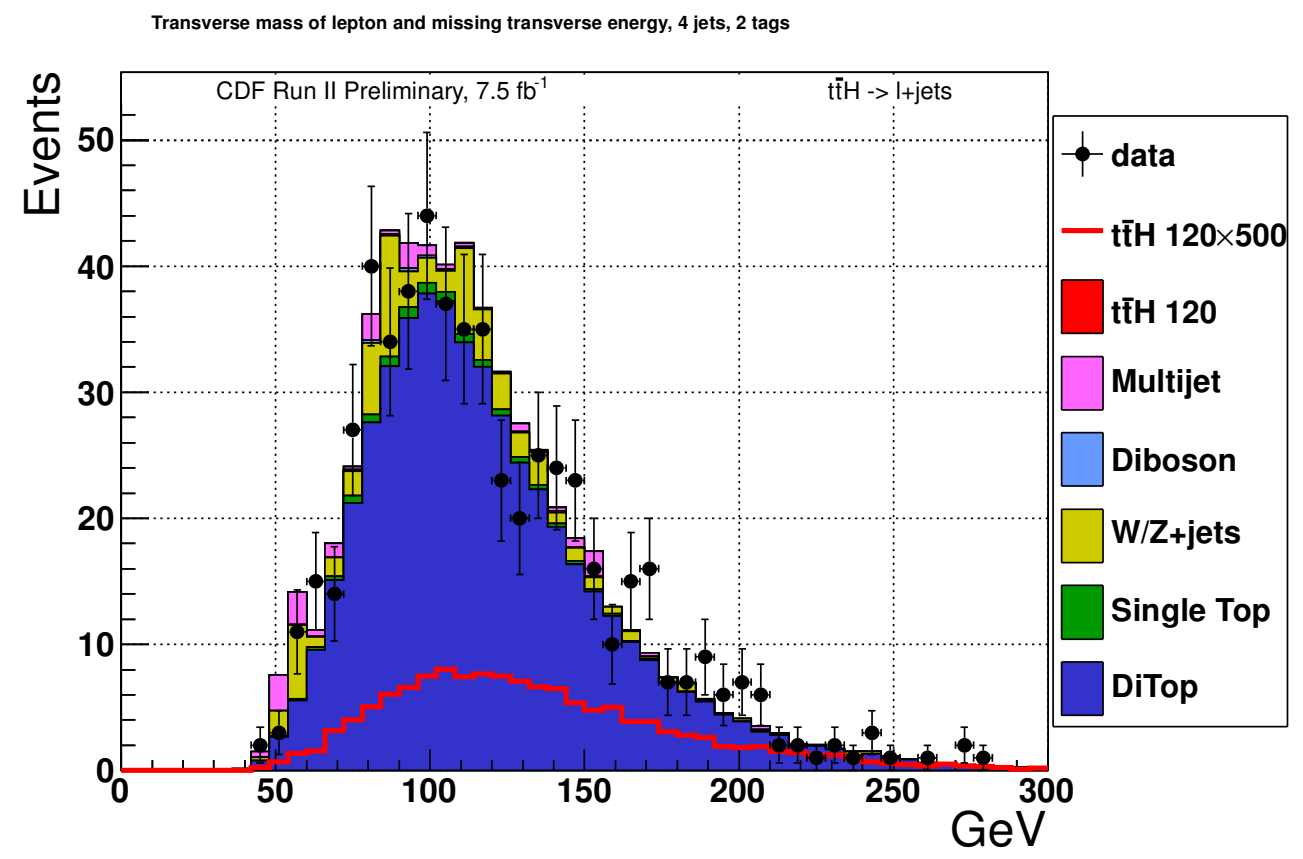

Figure 4.2: Leptonic $W$ transverse mass in the 4 jet, 2 tag sample

rate for data to obtain the tagged $W$ plus light flavor rate[43].

\subsection{Validating the background model}

As tables 4.1-4.5 show, our selected sample is overwhelming composed of $t \bar{t}$, which makes up no less than $81 \%$ of any analysis channel. We can verify these predictions by comparing the data to the full background prediction for various kinematic variables in each analysis channel.

Because $t \bar{t}$ events contain two real, on-shell $W$ bosons, we should be able to observe the signature of each, provided we can identify the decay products reliably. In 4 jet, 2 tag $t \bar{t}$ events, we expect the lepton and $E_{T}$ to represent the one $W$ boson, the two tagged jets to represent the two $b$ quarks from the top decays, and the two untagged jets to originate from the second $W$ boson. In this case, we expect to be able to reconstruct the $W$ mass from the untagged dijet. Since we do not know the neutrino momentum along the beamline $p_{z}$, we reconstruct the leptonic $W$ transverse mass. The data and background model for these 


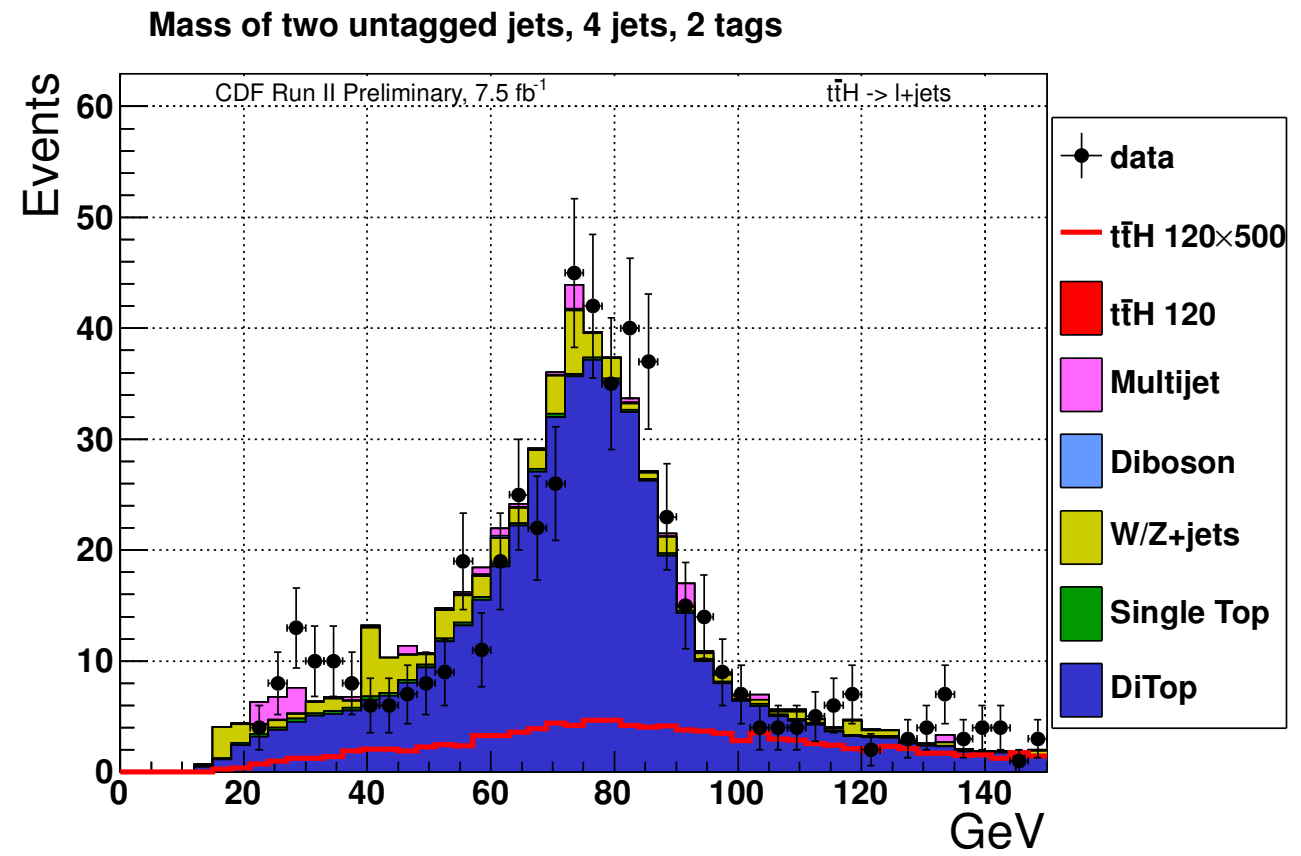

Figure 4.3: Hadronic $W$ dijet mass in the 4 jet, 2 tag sample

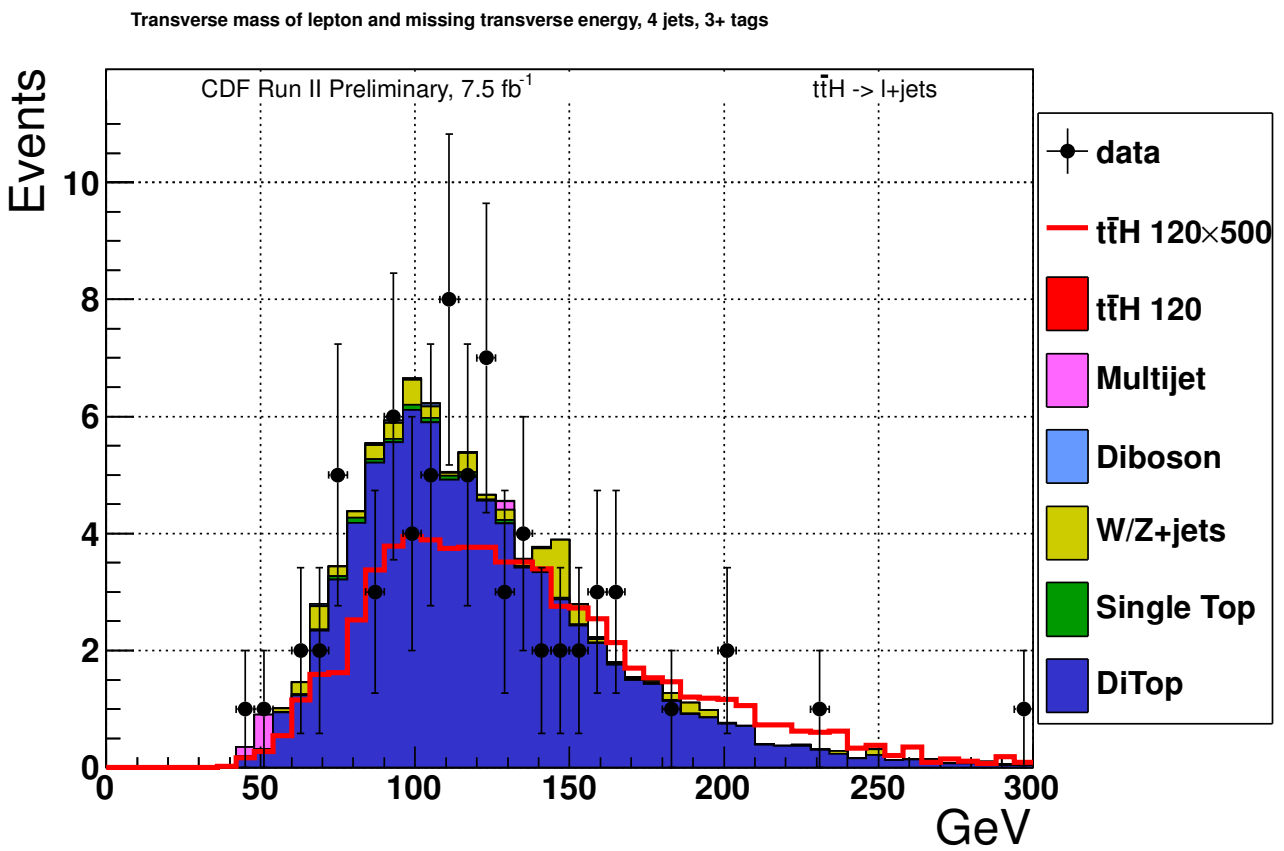

Figure 4.4: Leptonic $W$ transverse mass in the 4 jet, 3 tag sample 


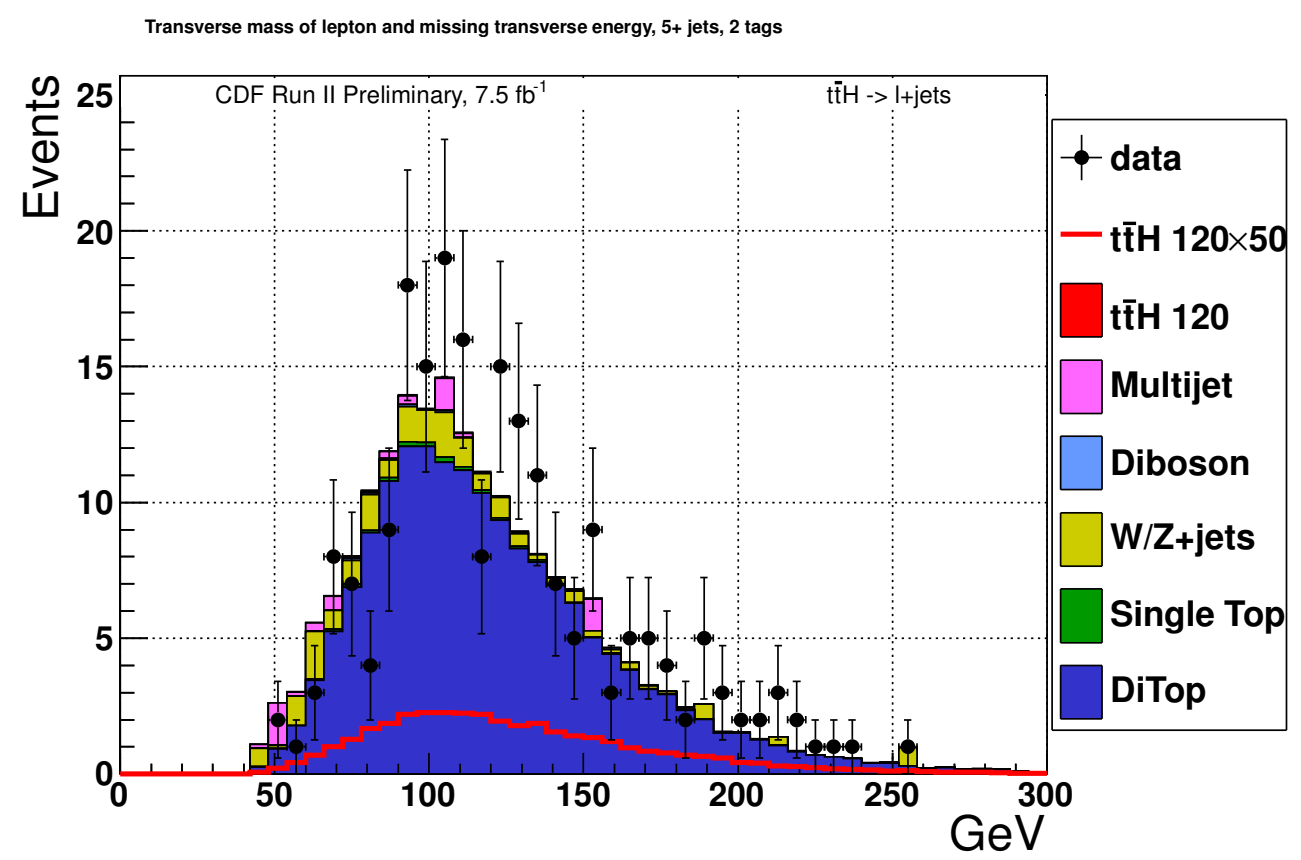

Figure 4.5: Leptonic $W$ transverse mass in the 5 jet, 2 tag sample

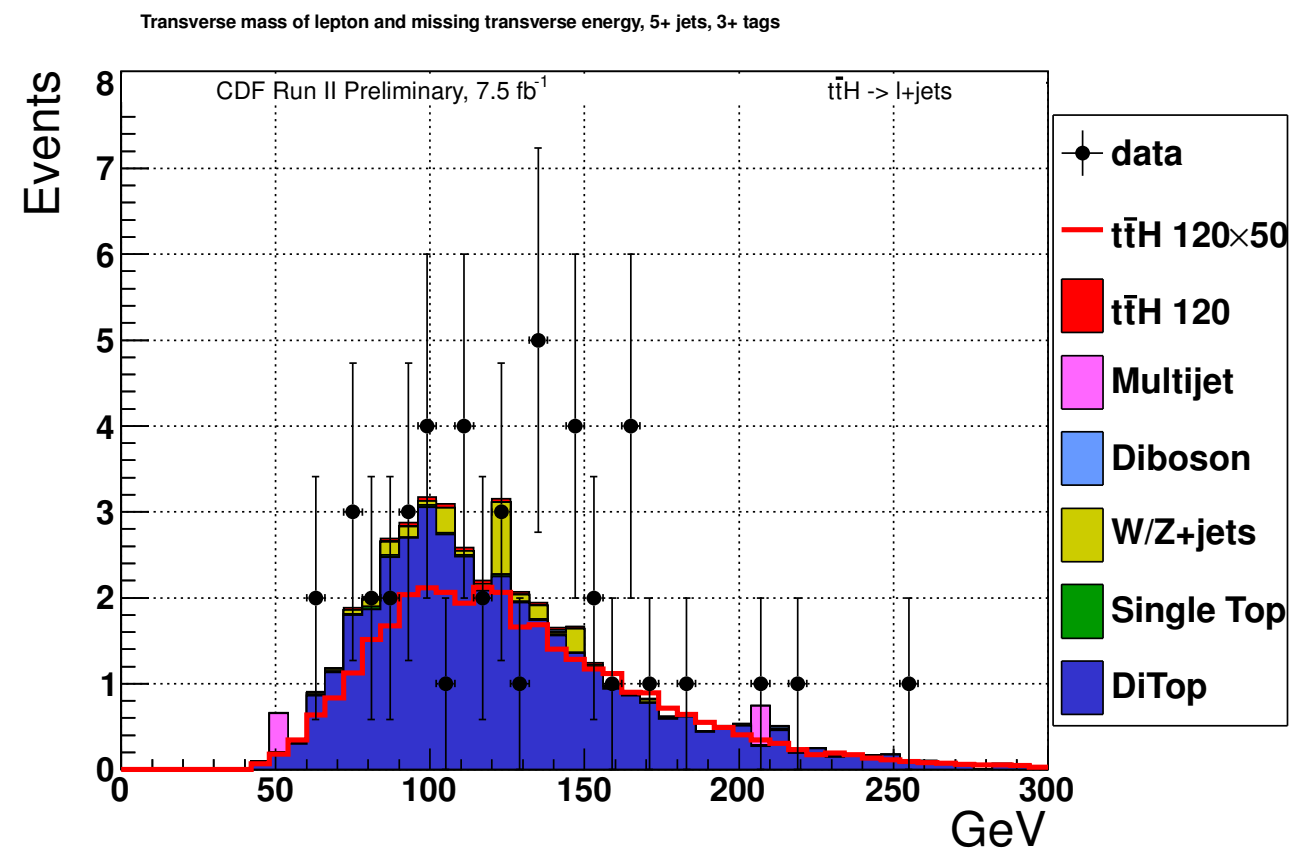

Figure 4.6: Leptonic $W$ transverse mass in the 5 jet, 3 tag sample 
Mass of two untagged jets, $5+$ jets, 2 tags

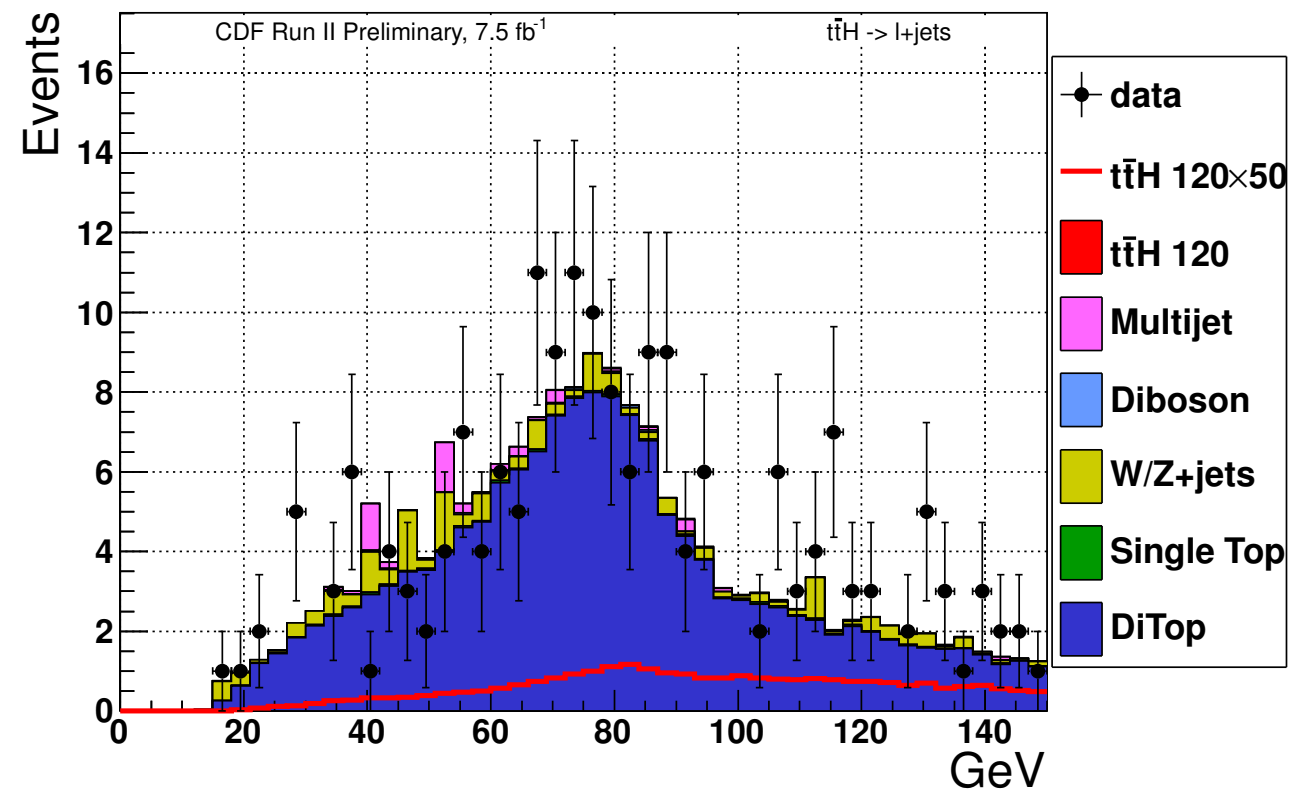

Figure 4.7: Hadronic $W$ dijet mass in the 5 jet, 2 tag sample

Mass of two untagged jets, $5+$ jets, $3+$ tags

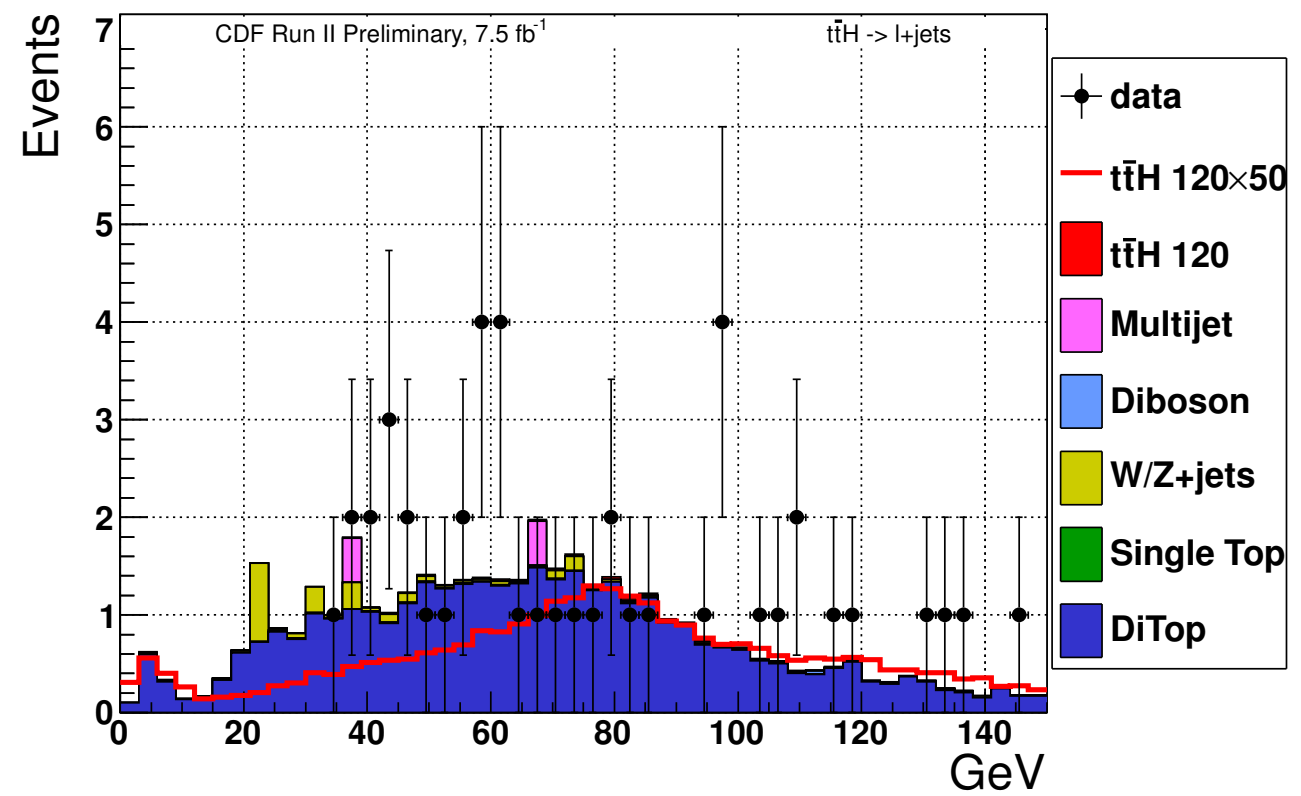

Figure 4.8: Hadronic $W$ dijet mass in the 5 jet, 3 tag sample 
variables in the 4 jet, 2 tag channels are shown in figures 4.2 and 4.3 . In the 5 or more jet, 2 tag and 3 or more tag samples, we can also reconstruct the hadronically-decaying $W$ boson with less reliability. Figures 4.7 and 4.8 show this. In the 4 jet, 3 or more tag sample, where there is only one untagged jet, this is not a useful validation tool, but the transverse mass of the leptonic $W$ boson (figure 4.4, 4.5, and 4.6) is useful in all channels. It is clear that our prediction that $t \bar{t}$ dominates this data sample is correct. 


\section{Chapter 5}

\section{DISCRIMINANTS}

Because the $t \bar{t} H$ signal is expected to exist at a rate enormously lower than the rate of our backgrounds, we do not perform a simple counting experiment. We can obtain much better sensitivity by using a binned likelihood in a variable that separates the signal from the backgrounds. In order to obtain a variable that separates the signal from the backgrounds very well, we combine a number of discriminating variables together using a supra-Bayesian ensemble of neural networks[44].

\subsection{Discriminating variables}

We examined a large number of kinematic variables that describe the events in our data sample, and selected a set of 20 that show some separation between the $t \bar{t} H$ signal and
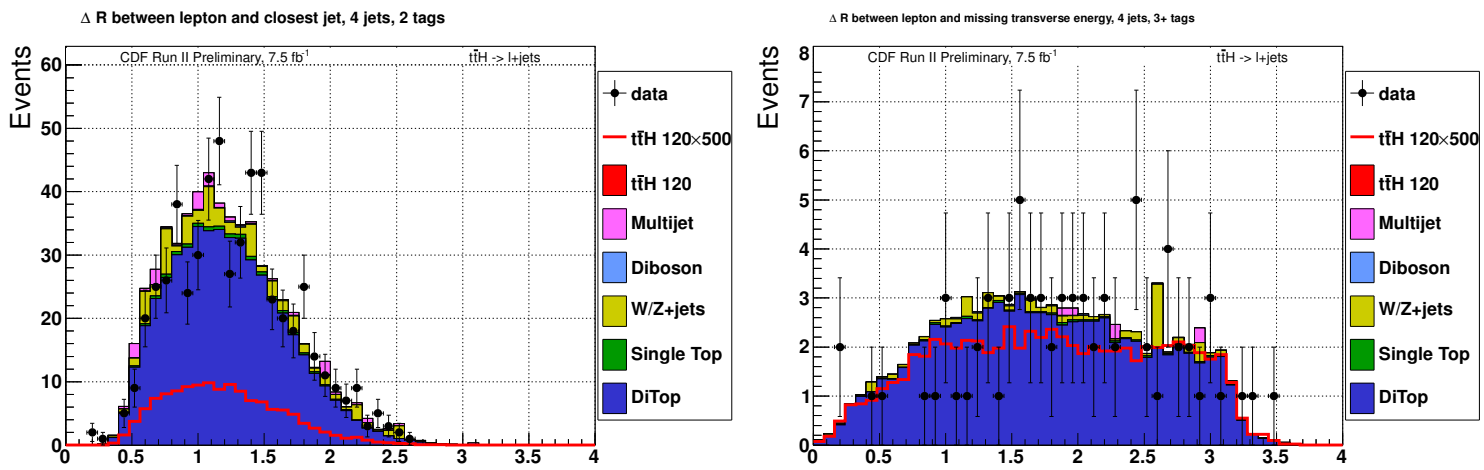

Figure 5.1: left: $\Delta R$ between lepton and closest jet (4 jets, 2 tags), right: $\Delta R$ between lepton and $\boldsymbol{E}_{\boldsymbol{T}}$ (4 jets, $3+$ tags) 

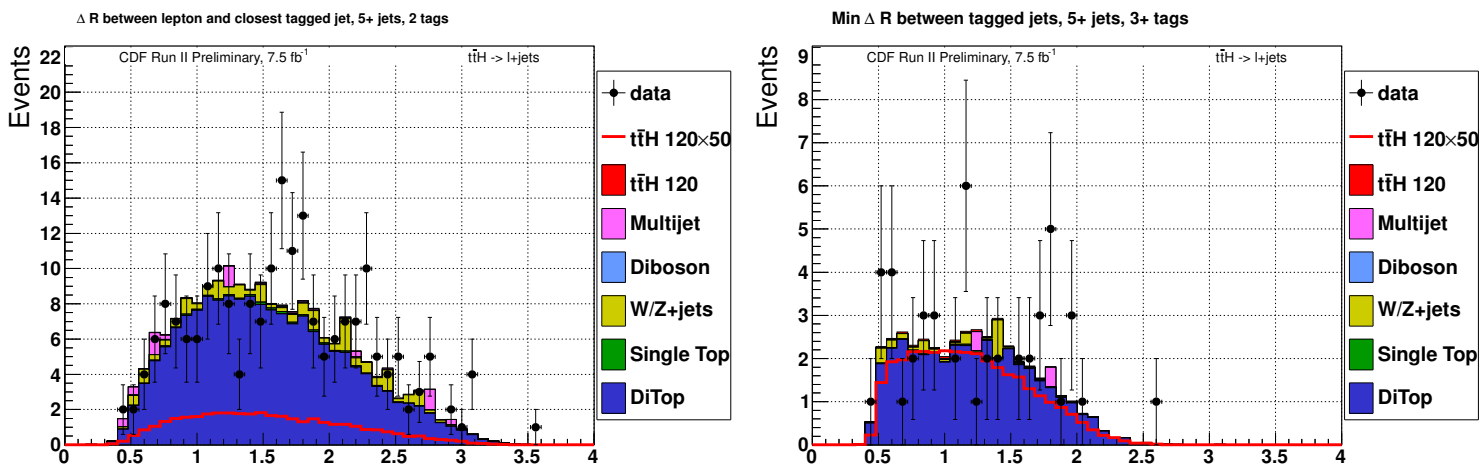

Figure 5.2: left: $\Delta R$ between lepton and closest tagged jet (5+ jets, 2 tags), right: $\min \Delta R$ between tagged jets $(5+$ jets, $3+$ tags $)$
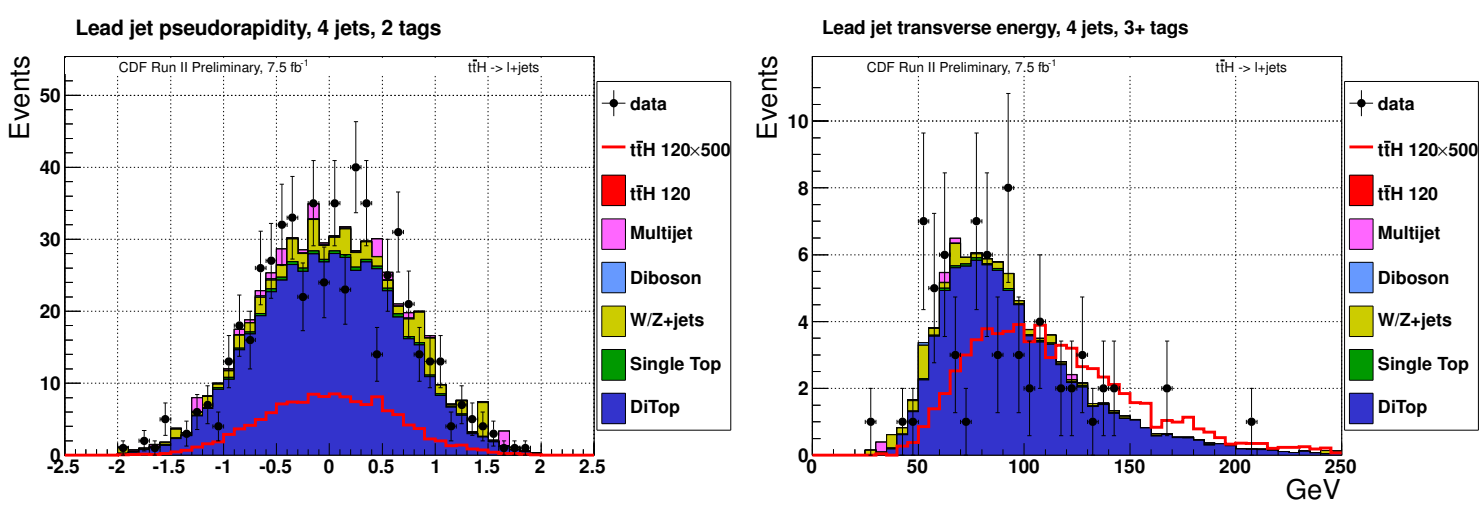

Figure 5.3: left: Lead jet pseudorapidity (4 jets, 2 tags), right: Lead jet transverse energy (4 jets, $3+$ tags)
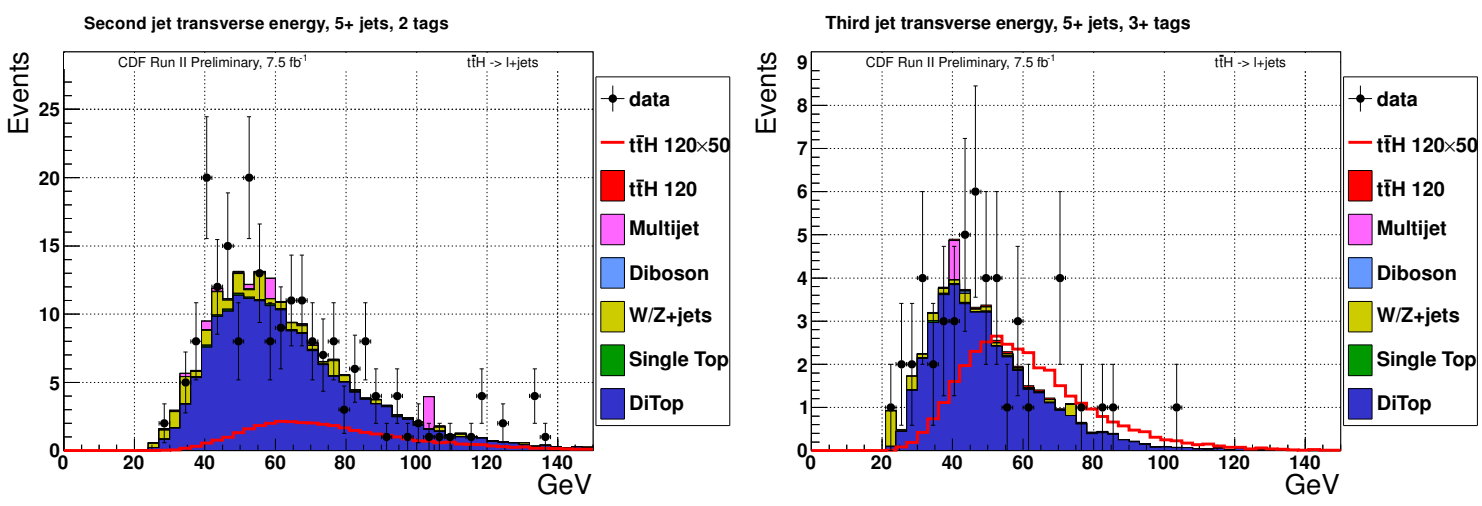

Figure 5.4: left: Second jet $E_{T}(5+$ jets, 2 tags $)$, right: Third jet $E_{T}(5+$ jets, $3+$ tags $)$ 

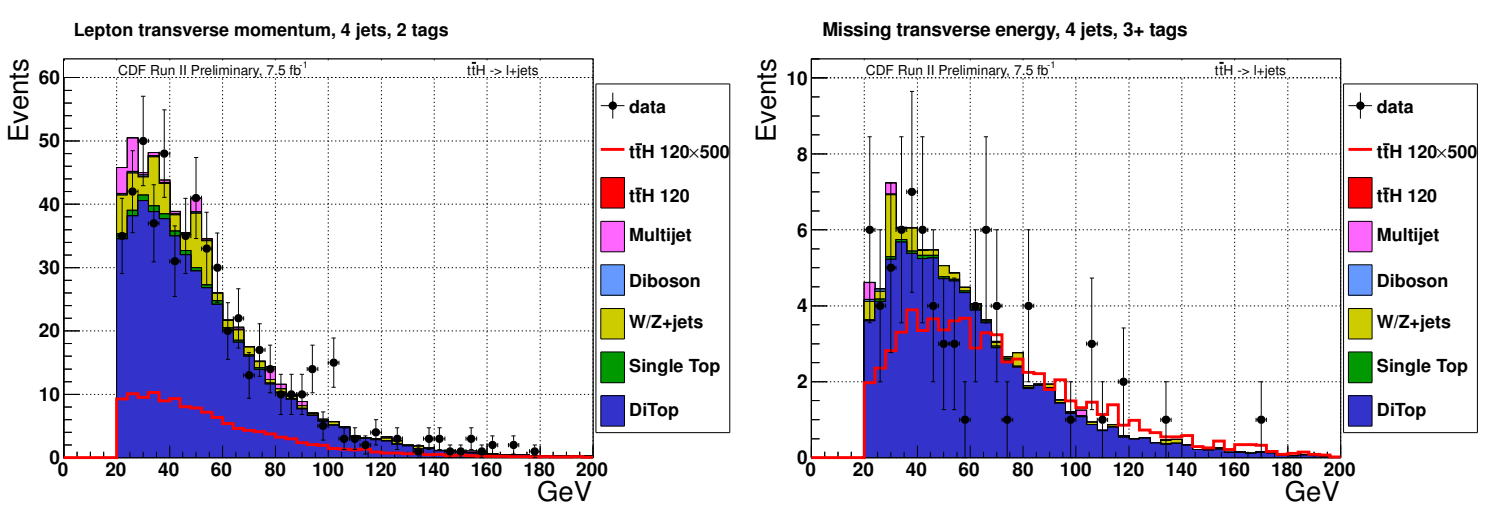

Figure 5.5: left: Lepton $p_{T}$ (4 jets, 2 tags), right: $\mathbb{E}_{T}$ (4 jets, $3+$ tags $)$
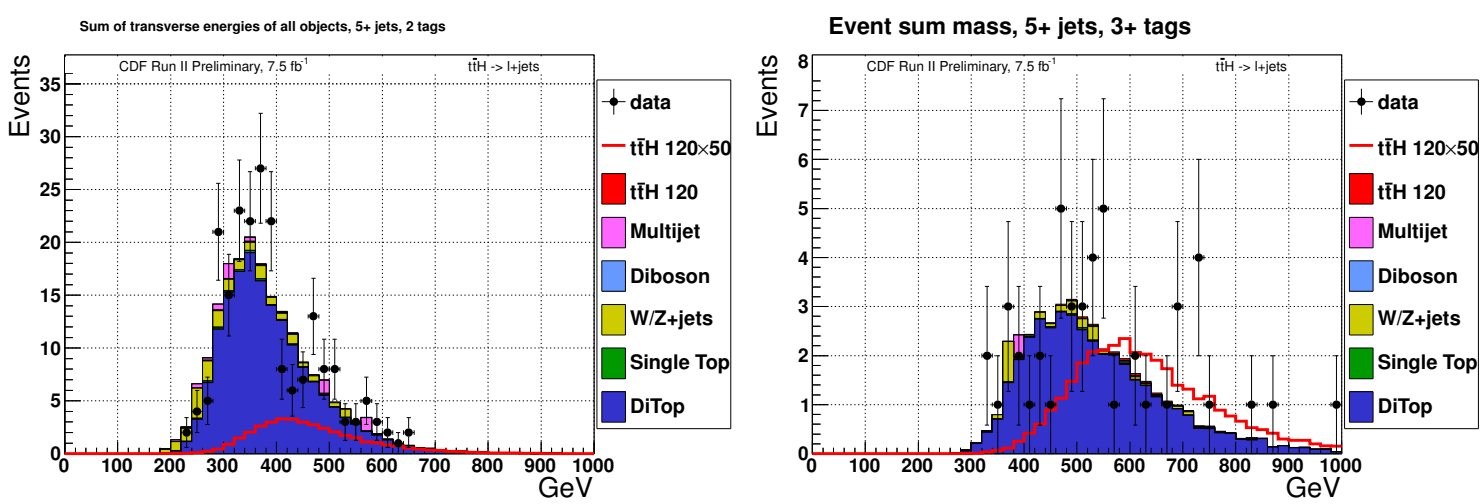

Figure 5.6: left: $H_{T}(5+$ jets, 2 tags $)$, right: Mass of all event objects $(5+$ jets, $3+$ tags $)$
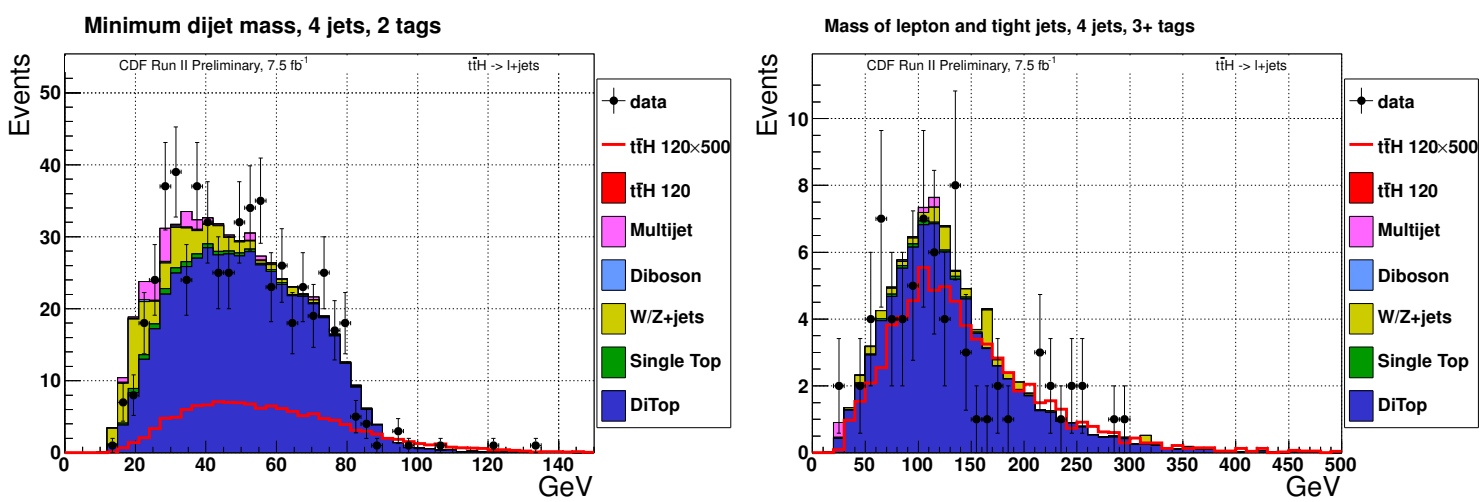

Figure 5.7: left: Minimum dijet mass (4 jets, 2 tags), right: Mass of lepton and jets (4 jets, $3+$ tags) 

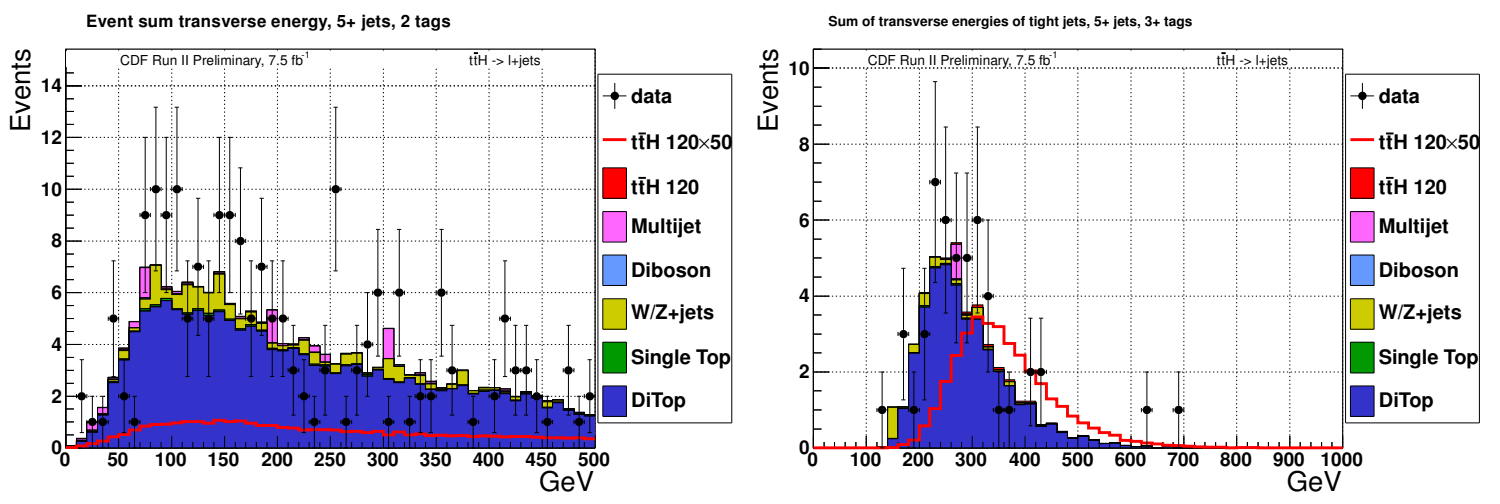

Figure 5.8: left: Transverse energy of all event objects $(5+$ jets, 2 tags $)$, right: $\sum_{\text {jets }} E_{T}(5+$ jets, $3+$ tags)
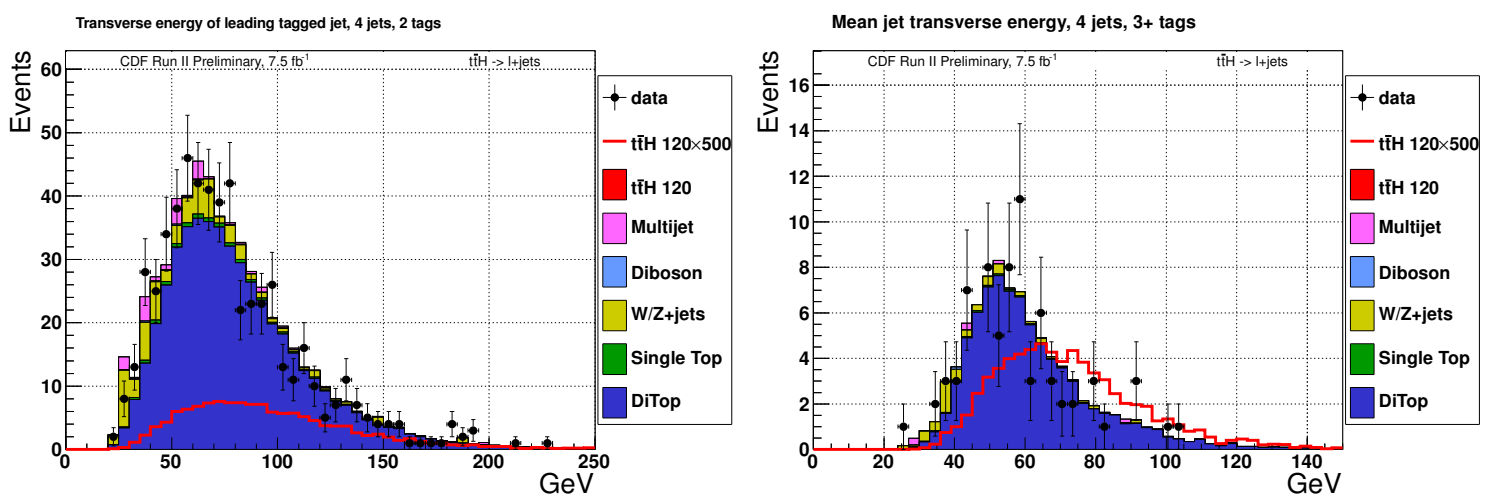

Figure 5.9: left: Maximum $E_{T}$ of tagged jets ( 4 jets, 2 tags), right: Mean jet $E_{T}(4$ jets, $3+$ tags) 
dominant $t \bar{t}$ background:

- $E_{T}$

- $\min \Delta R$ between tagged jets $\left(\Delta R \equiv \sqrt{\left(\phi_{1}-\phi_{2}\right)^{2}+\left(\eta_{1}-\eta_{2}\right)^{2}}\right)$

- $\Delta R$ between lepton and nearest jet

- $\Delta R$ between lepton and nearest tagged jet

- $\Delta R$ between lepton and $\mathbb{E}_{T}$

- $W\left(\ell+E_{T}\right)$ transverse mass

- Mass of highest $E_{T}$ pair of untagged jets

- Minimum dijet mass

- Mass of all event objects $\left(\mathbb{E}_{T}\right.$, lepton, jets)

- Vector sum of transverse energies of all event objects

- Scalar sum of transverse energies of all event objects $\left(H_{T}\right)$

- Mass of lepton and jets

- $\sum_{\text {jets }} E_{T}$

- $\eta$ for highest $E_{T}$ jet

- Largest jet $E_{T}$

- Second largest jet $E_{T}$

- Third largest jet $E_{T}$

- Largest tagged jet $E_{T}$

- Mean jet $E_{T}$

- Lepton $p_{T}$

With any multivariate, computer-learning technique, it is important that all of the inputs be well modeled in order to prevent the training algorithm utilizing features of the background model that are not present in the data. A selection of these variables are shown to be well modeled in figures 5.1-5.9; the full set may be found in appendix A. 


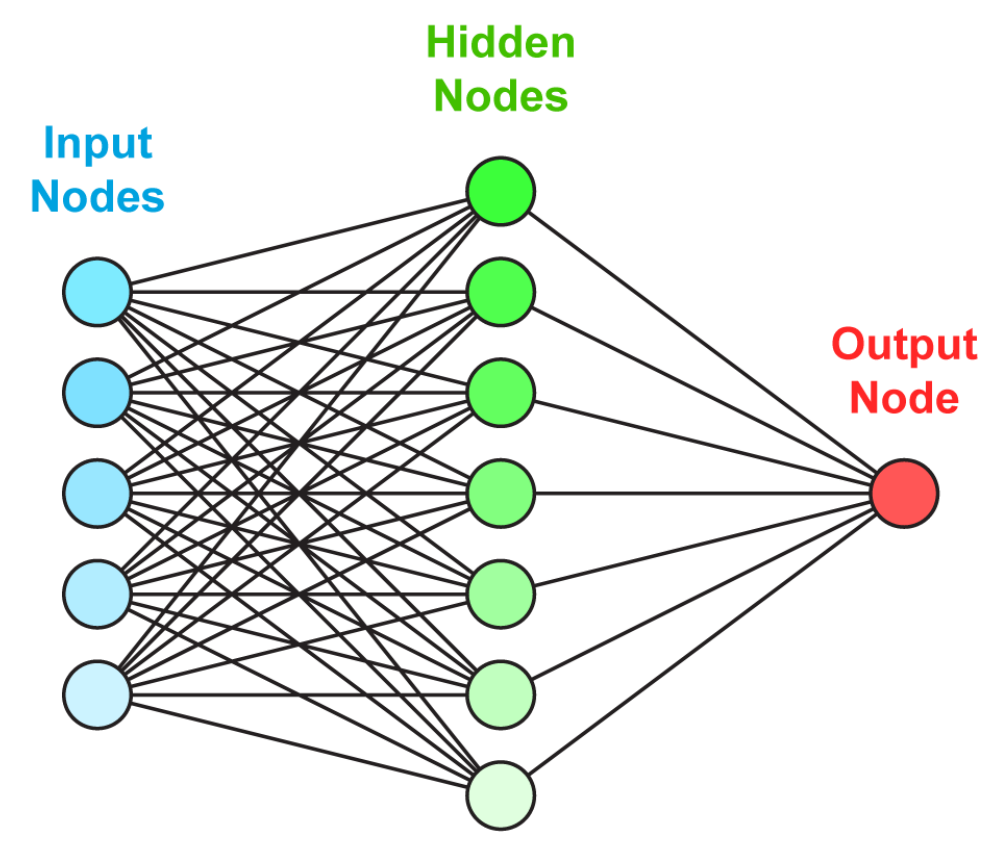

Figure 5.10: A feed-forward multilayer perception (neural network) architecture

\subsection{Neural Networks}

An artificial neural network is a simple computational model for the functioning of actual networks of neural cells in organisms. The class of artificial neural networks called feedforward multilayer perceptrons can approximate any mathematical function with arbitrary accuracy, and can be "trained" to approximate an extensionally defined function via an iterative procedure[45]. As a result, feed-forward multilayer perceptrons are widely used in science and engineering to perform regression and classification tasks.

Feed-forward multilayer perceptrons ("neural networks" in the rest of the document) are composed of a number of layers of "nodes" as shown in figure 5.10. The input layer consists of a number of nodes with a single input and multiplexed output, with one node per input variable. The next layer is called the "hidden" layer, and each hidden node takes as many inputs as there are nodes in the previous layer, and has one multiplexed output. There can be many hidden layers, but one hidden layer is sufficient to approximate any function to arbitrary accuracy. The final layer is the output layer, and has one node which takes inputs 
from the outputs of the previous layer and produces one output value for a given input vector.

Each node functions identically. A node has $N$ inputs $x_{i}$ and one output. Each input is multiplied by a weight $w_{i}$ and the weighted inputs are summed. This sum is transformed by a sigmoid function (called the "activation function") such as arctan to produce the output.

$$
f\left(x_{i} ; w_{i}\right)=\sigma\left(-t+\sum_{i=0}^{N} x_{i} w_{i}\right)
$$

The threshold of the activation function $t$ can also be altered, or an additional input node which always has 1 (a "bias node") as its input can be used. These two approaches to the activation function threshold are identical.

To "train" a neural network, a set of input vectors and desired output values are collected. The neural network initially is given randomized weights and activation thresholds. The output for each input vector is calculated and compared to the target output, and then the weights and thresholds are adjusted to reduce the aggregate error. This process is repeated (each iteration is an "epoch") until the weights and thresholds begin to converge. The exact method used in this analysis for adjusting the weights and thresholds is the Broyden-Fletcher-Goldfarb-Shanno method, which approximates Newton's method for finding a local minimum[46].

\subsection{Ensemble Method}

Because multivariate technique training is a non-deterministic process, there is some variation in the result of a multivariate analysis if the analysis is repeated including re-training. In order to control this variation and make our result more repeatable, we use an ensemble of 1000 neural networks for a discriminant. We train a separate ensemble discriminant for each of the 12 candidate Higgs masses in our signal model.

We use our $t \bar{t}$ and $t \bar{t} H$ Monte Carlo as training samples. A random subset of the Monte Carlo samples with 5 or more jets and 2 or more tags is selected for training and a non-overlapping set for testing. Ten of the 20 candidate input variables are randomly 
selected and used as the inputs to a neural network like that shown in figure 5.10. This random selection of training and testing samples and input variables is repeated for each neural network training. The histograms describing the output of each network on the testing sample are normalized to 1 and stored along with the network.

To evaluate the 1000 network ensemble on a novel event, we look at each constituent network in turn. We evaluate the network, and then find the bin in the stored testing histograms for signal and for background where that output falls. The fraction of the signal testing set falling in that bin is divided by the fraction of the background testing set falling in the same bin is the figure of interest for that network. This process is repeated for each network in the ensemble, and the average of the signal fraction to background fraction ratio is averaged. This average is the output of the ensemble[44].

\subsection{Discriminant outputs}

The discriminant outputs for a candidate Higgs boson mass $m_{H}=115 \mathrm{GeV} / c^{2}$ are shown in figure 5.11. The output is well modeled, as we expected since the inputs are also well modeled. The discriminants perform very well in the 5 jet channels, where they separate the $t \bar{t} H$ signal from the $t \bar{t}$ background much better than any individual input variable. In the $5+$ jet, $3+$ tag channel, the signal to background ratio in the most signal-like bin is $\sim 0.08$.

In the 4 jet channels, the performance is not as strong. These channels contain very little signal, and the discriminants were not trained on 4 jet events, but there is still some useful separation. The 4 jet channels serve principally as a control region to constrain the $t \bar{t}$ rate for the 5 jet channels.

By providing a set of bins with widely varying signal to background ratios and increasing the maximum signal to background ratio, these discriminants will improve the sensitivity of our analysis over using any single kinematic variable or a simple one-bin counting experiment. 

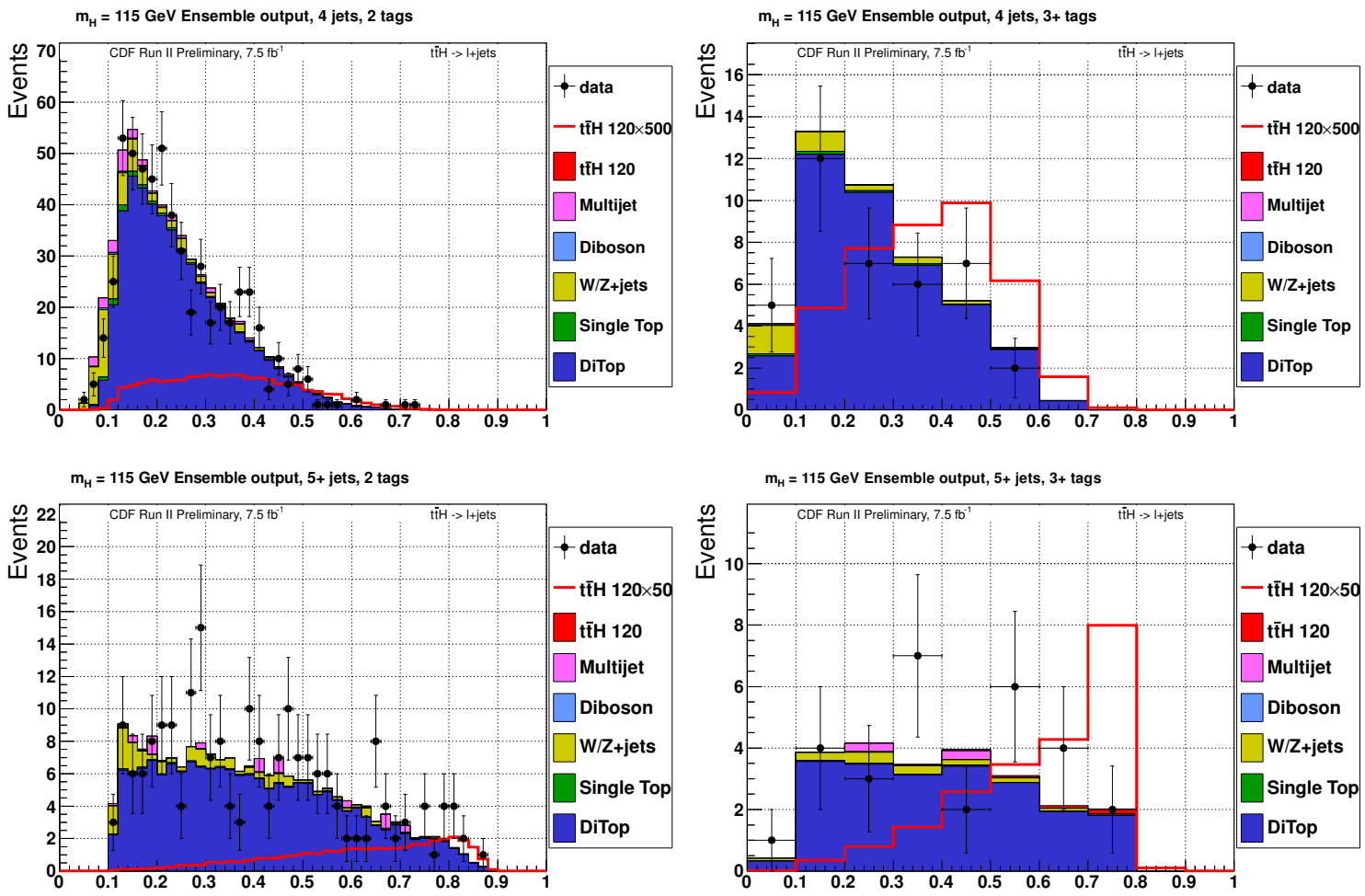

Figure 5.11: Discriminant outputs for $m_{H}=115 \mathrm{GeV} / c^{2}$ 


\section{Chapter 6 Systematic UnCeRTAinties}

We deal with a number of sources of systematic uncertainty. The major categories of uncertainty sources are a lack in our technical ability to calculate theoretical predictions, an imperfect knowledge of the fundamental parameters of the theory, and a mismeasurements by the detector.

In calculating the upper bound on the rate at which the Higgs boson could be present in our data without observable inconsistencies with our background model, we take the various systematic uncertainties into account by parameterizing them and their effects on our predictions, and then performing a Monte Carlo integration over these parameters (see section 7.1). In order to accomplish this parameterization, we first estimate the change in rate and shape of the discriminating variables due to shifts in the systematic parameters.

\subsection{Initial- and Final-State Radiation}

Any colored particle, whether in an initial, intermediate, or final state, can radiate any number of gluons. When this radiation is at a high enough energy, the gluon may form a jet or may split to a quark pair and form two or more jets. This can affect the number of jets and the number of $b$ tags in the event, which would change which analysis channel the event falls into. It can also affect various quantities that are used as inputs to the discriminants, such as energy sums like $H_{T}, \mathbb{E}_{T}$, and geometric separation variables like $\Delta R$ between the lepton and nearest jet. Radiation can even affect the lepton acceptance rate by causing leptons to be non-isolated. 

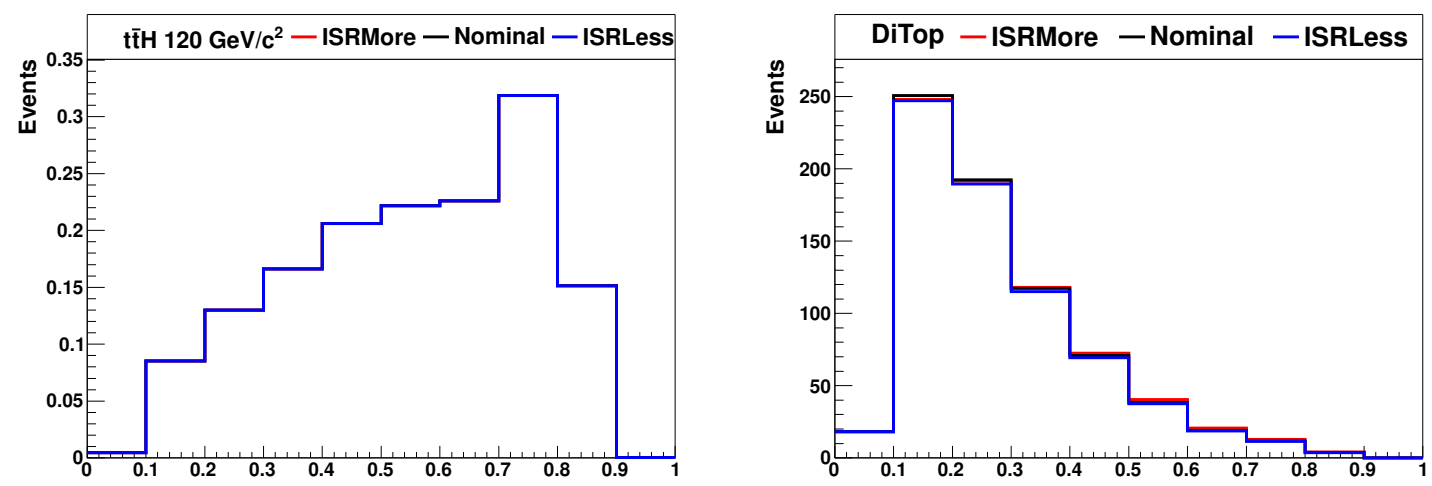

Figure 6.1: Effect of initial- and final-state radiation on discriminant output for $t \bar{t} H$ signal and $t \bar{t}$ background - effect on $t \bar{t} H$ signal is negligible
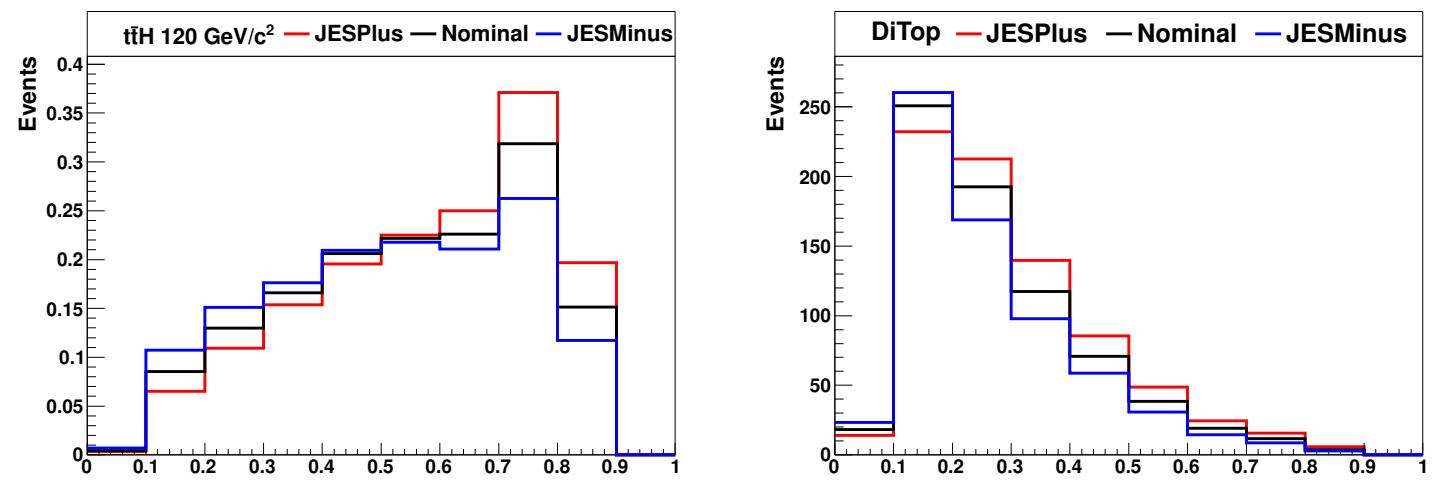

Figure 6.2: Effect of JES shifts on discriminant output for $t \bar{t} H$ signal and $t \bar{t}$ background

We handle the effects of the ISR/FSR systematic by generating multiple Monte Carlo samples for both our signal and our largest background, $t \bar{t}$, with varied radiation amounts. We run the full analysis on these samples, generating discriminant output histograms for each, shown in figure 6.1. These histograms are used to account for the change in both the rate of the signal and dominant background and the shape of their distributions in the discriminant output. 


\subsection{Jet Energy Scale}

There are a large number of contributions to the jet energy scale uncertainty, which are detailed in section 3.3. The jet energy scale uncertainty is the residual uncertainty in the energies of jets after correcting for all of these effects.

We handle the effects of this systematic by evaluating not only the magnitude of the energy corrections that we apply to each jet, but also the residual uncertainty on each correction factor. We then run the analysis on each Monte Carlo sample three times: once we use the nominal jet energy correction factor, once we use the nominal correction factor

plus the uncertainty, and once we use the nominal correction factor minus the uncertainty. This generates three discriminant output histograms for each Monte Carlo sample, shown in figure 6.2 , which we again use to account for the effects on the final result of both the change in the rate and the shape of the signal and all of the backgrounds.

Shifts in the jet energy scale affect the analysis in similar ways to the ISR/FSR systematic shifts. By changing the energies of jets, all quantities which are related to the jet energies, such as the lead and second jet $E_{T}$, are changed. Additionally, changing jet energies may move low energy cluster up to high enough energies to meet our jet definition, or vice versa, causing variables related to the number of jets to change as well.

\subsection{Mistag rate}

The mistag matrix is used to evaluate the probability that a light flavor jet in Monte Carlo would be tagged, and this probability is used to weight Monte Carlo events when making histograms. The mistag rates are not known a priori and must be measured in a control region. The mistag systematic uncertainty accounts for variation, both statistical and systematic, in the measurement of the mistag rates. Like the ISR/FSR and JES systematics, the mistag systematic is a rate and shape systematic. The shifted and nominal shapes are shown in figure 6.3. 

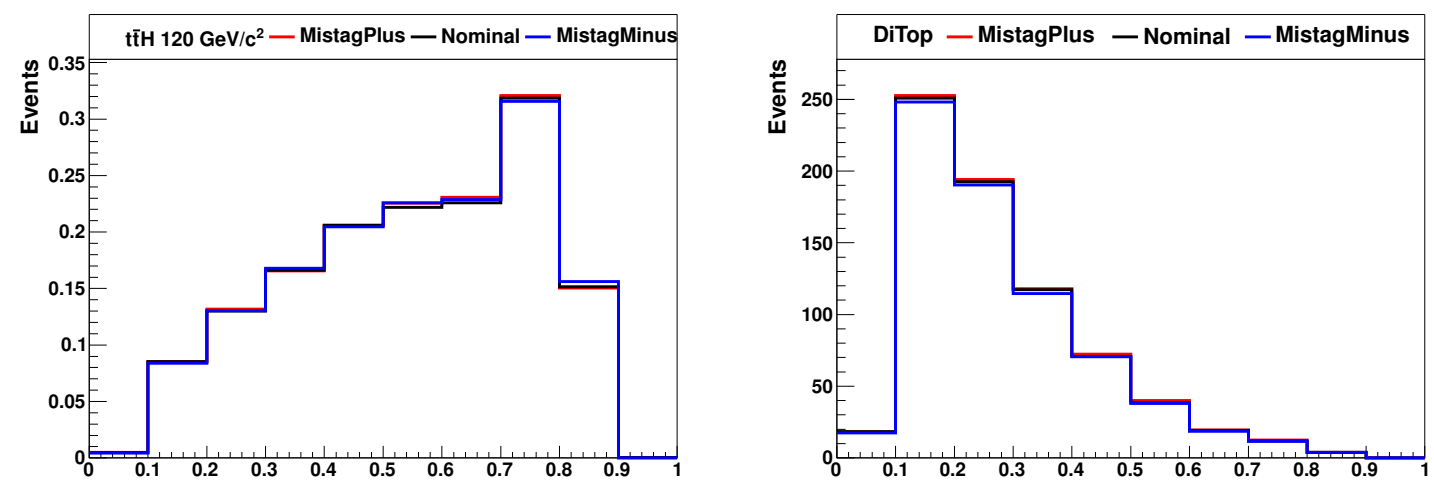

Figure 6.3: Effect of shifts in the mistag rate on discriminant output for $t \bar{t} H$ signal and $t \bar{t}$ background
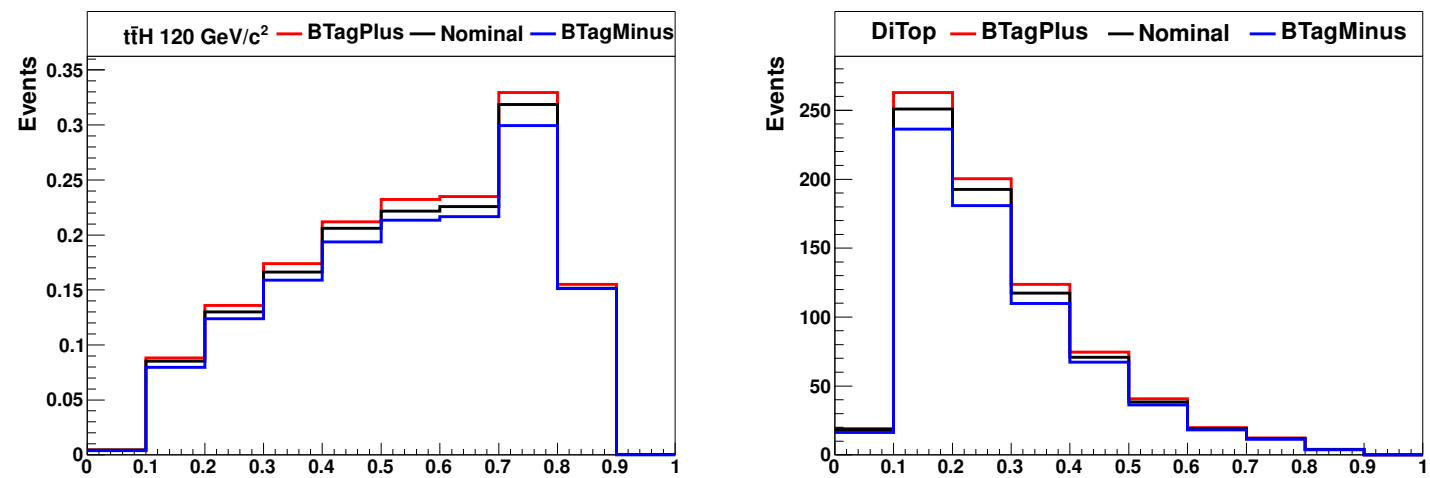

Figure 6.4: Effect of shifts in the $b$-tagging scale factor on discriminant output for $t \bar{t} H$ signal and $t \bar{t}$ background

\section{4 b-tagging scale factor}

The $b$-tagging scale factors are used to account for differences between the tagging rate for heavy flavor jets in Monte Carlo and in data. Like the mistag rates, the scale factors must be measured, and some uncertainty is associated with such a measurement. The $b$-tag scale factor systematic is also a rate and shape systematic, with the shifted and nominal shapes shown in figure 6.4, and accounts for the uncertainty in the scale factor measurements. 


\subsection{Cross section systematics}

Cross section systematics are an example of a systematic arising from our inability to calculate the predictions of the Standard Model perfectly. We apply a $10 \%$ systematic uncertainty to the cross section for $t \bar{t}$ and for $t \bar{t} H$. We also have cross section uncertainties for other processes: $10 \%$ for single top, $6 \%$ for diboson, and $40 \%$ for the $k$-factor applied to the $W$ plus heavy flavor cross sections. These uncertainties do not affect the shape of the discriminant output distributions, but only the total rates for the signal and backgrounds[47].

\subsection{Luminosity}

We measure the instantaneous luminosity delivered by the Tevatron to CDF using the Cherenkov Luminosity Counter. The Fermilab accelerator division also measures the Tevatron beam current and focusing, giving a second measurement of the luminosity. As with any other measurement, there is some uncertainty associated with the measurement. We apply a $4.4 \%$ rate uncertainty to every process based on the CDF luminosity measurement, and an additional $3.8 \%$ rate uncertainty based on the Tevatron luminosity measurement.

\subsection{Summary}

Tables 6.1 and 6.2 show the full effects of these systematic uncertainties on the $t \bar{t} H$ signal and the $t \bar{t}$ background in each of the different tagging categories. 


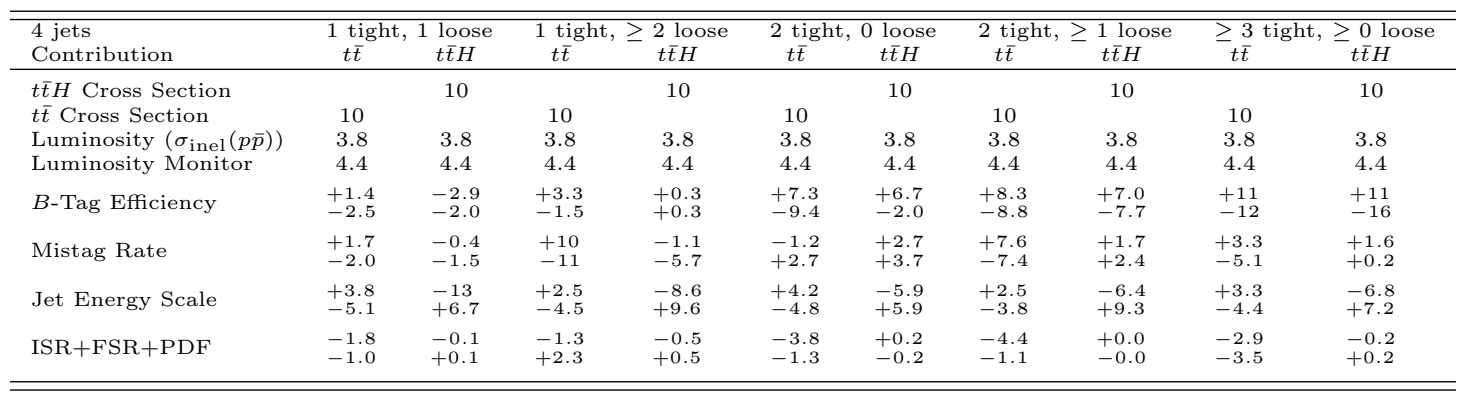

Table 6.1: Effects of the systematic uncertainties on the $t \bar{t} H$ signal $\left(m_{H}=115 \mathrm{GeV} / c^{2}\right)$ and $t \bar{t}$ background for the 4 -jet channels. Uncertainties are relative, in percent, and are symmetric unless otherwise indicated.

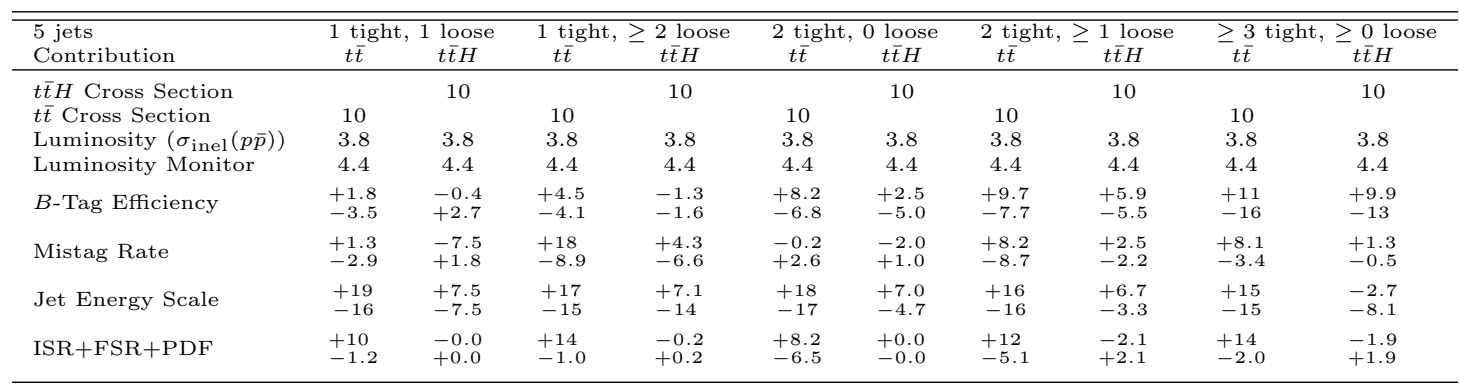

Table 6.2: Effects of the systematic uncertainties on the $t \bar{t} H$ signal $\left(m_{H}=115 \mathrm{GeV} / c^{2}\right)$ and $t \bar{t}$ background for the 5 -jet channels. Uncertainties are relative, in percent, and are symmetric unless otherwise indicated. 


\section{Chapter 7 RESULTS}

Having selected a sample of events expected to contain the $t \bar{t} H$ process if it exists, modeled the expected backgrounds in that sample, developed a set of variables designed to isolate $t \bar{t} H$ from the backgrounds as much as possible, and evaluated the effects of uncertainties in our measurements and calculations, we would like to establish whether or not the $t \bar{t} H$ process does exist. Since we do not observe any significant excess of data over our predicted backgrounds, particularly in the most signal-like regions, we will proceed to set upper bounds on the amount of signal that could be present in our data.

\subsection{Limit calculation}

The simplest procedure for calculating upper limits is a counting experiment. We can predict the total amount of various background that should fall into our data sample, calculate the Poisson uncertainty and the systematic uncertainties on the total rate, and compare it to our data. For some examples, we will perform such a counting experiment with statistical errors alone. The rate at which events fall into our selection is Poisson distributed, and so we use the square root of the number of events as the statistical uncertainty, $\sigma$.

Examining tables 4.1-4.5, we have a total of 907.59 predicted background events and 1.83 predicted signal events for a Higgs mass of $120 \mathrm{GeV} / c^{2}$. If the signal were larger by a factor of 33 than our prediction, then we would predict $907.59+33 \cdot 1.83=967.98$ events, which is $2 \sigma$ higher than the 907.59 events we would expect to see in the absence of any signal. We would expect, then, to exclude this possibility with greater than $95 \%$ confidence. 
Since we observe only 892 events, which is fewer than the 907.59 predicted events, we are able to actually exclude the possibility that the signal is produced only 24 times as much as predicted with $95 \%$ confidence.

Since the signal to background ratio in the 5 or more jet and 3 or more tag categories is so much better than in the 4 jet or 2 tag categories, it is natural to attempt to separate these to improve the sensitivity of the analysis. If we take the single best tagging category, 4 or more jets and 3 or more SECVTX tags, we predict 27.16 background and 0.37 signal events, and observe 25 events in the data. With this sample, we would expect to exclude a signal production rate of $\frac{\sqrt{27.16} \cdot 1.96}{0.37}=27.6$ times the Standard Model predicted rate with $95 \%$ confidence, and, using our data, we observe an exclusion of 20.6 times the Standard Model prediction. This is much improved over the counting experiment using the 2 tag categories, but it discards a significant amount of data and predicted signal.

In order to include the additional data, we can perform a two-channel counting experiment. We combine two channels by adding their limits in inverse quadrature, since the statistical errors are completely uncorrelated. If we were to perform this combination in the presence of systematic uncertainties, we would have to use a more complicated procedure to correctly account for correlations in the errors.

The 4 or more jets, 2 tag sample contains a predicted 776.95 background and 1.01 signal events, and we observe 771 data events. Using this, we expect a limit at 54.1 times the Standard Model, and observe a limit at 48.0 times the Standard Model. Combining this with the 4 or more jets, 3 or more tags sample gives us a combined expected limit of $\frac{1}{\sqrt{1 / 54.1^{2}+1 / 27.6^{2}}}=24.6$ times the Standard Model, and a combined observed limit of $\frac{1}{\sqrt{1 / 48.0^{2}+1 / 20.6^{2}}}=18.9$ times the Standard Model. This is better than either channel separately, and much better than simply joining these channels into a single counting experiment.

The success of such a combination indicates that further division is not unwarranted. In the real analysis, we divide our sample, as mentioned, into 10 different channels. Additionally, since we do not perform a simple counting experiment, even in those 10 channels, our binned likelihood method is akin to dividing each of the 10 channels into 10 to 50 subchannels 
(depending on the number of bins used in the histograms) with correlated errors.

\subsubsection{Bayesian limit calculation}

We use the mclimit Bayesian limit calculator[48] to perform the full estimation of systematic uncertainties, including correlations, and the combination of limits among multiple channels. Mclimit is also used to calculate the full CDF and Tevatron combined limits on Higgs production.

As in the multiple-channel counting experiment above, we combine all the bins of the discriminant histograms in all the channels using a joint Poisson probability, given certain shifts in the systematic uncertainties. Then, by integrating over the prior probabilities for the systematics, we can obtain a posterior probability for any signal cross section. By integrating that posterior distribution, we can find the $95 \%$ confidence upper bound on the cross section as a multiple of the Standard Model prediction.

These integrals are impossible to perform analytically, so we perform them using a Monte Carlo integration technique. In order to estimate the sensitivity of the analysis before we look at the data, we also perform a Monte Carlo integration over the number of background events in each bin, using Poisson distributions for the priors. This enables us to predict the range that we would expect the observed limit to fall in once we do look at the data.

\subsection{Results}

The $95 \%$ confidence level upper bounds on the $t \bar{t} H$ cross section as a multiple of the Standard Model predicted cross section are shown in figure 7.1 for the 4 jet and 5 or more jet channels separately, and in figure 7.2 and table 7.1 for the entire analysis combined. At a Higgs mass of $120 \mathrm{GeV} / c^{2}$, we expect to set a limit of 12.7 times the Standard Model prediction in the absence of a Higgs boson, with a $1 \sigma$ interval from 8.7 to 19.1, and a $2 \sigma$ interval from 6.3 to 26.7. So, if we were to repeat the entire experiment many times, taking entirely new data, we would expect the observed limit to fall between 8.7 and $19.168 \%$ of the time, and between 6.3 and $26.795 \%$ of the time, provided that the Higgs boson does not exist. We 

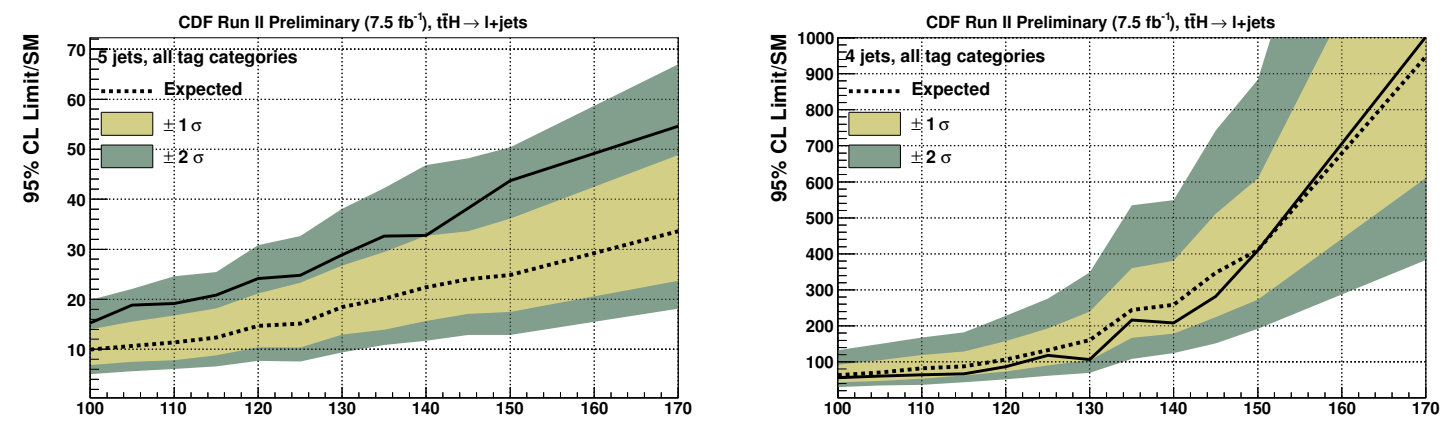

Figure 7.1: $95 \%$ confidence level limits with 5 or more jets and with 4 jets

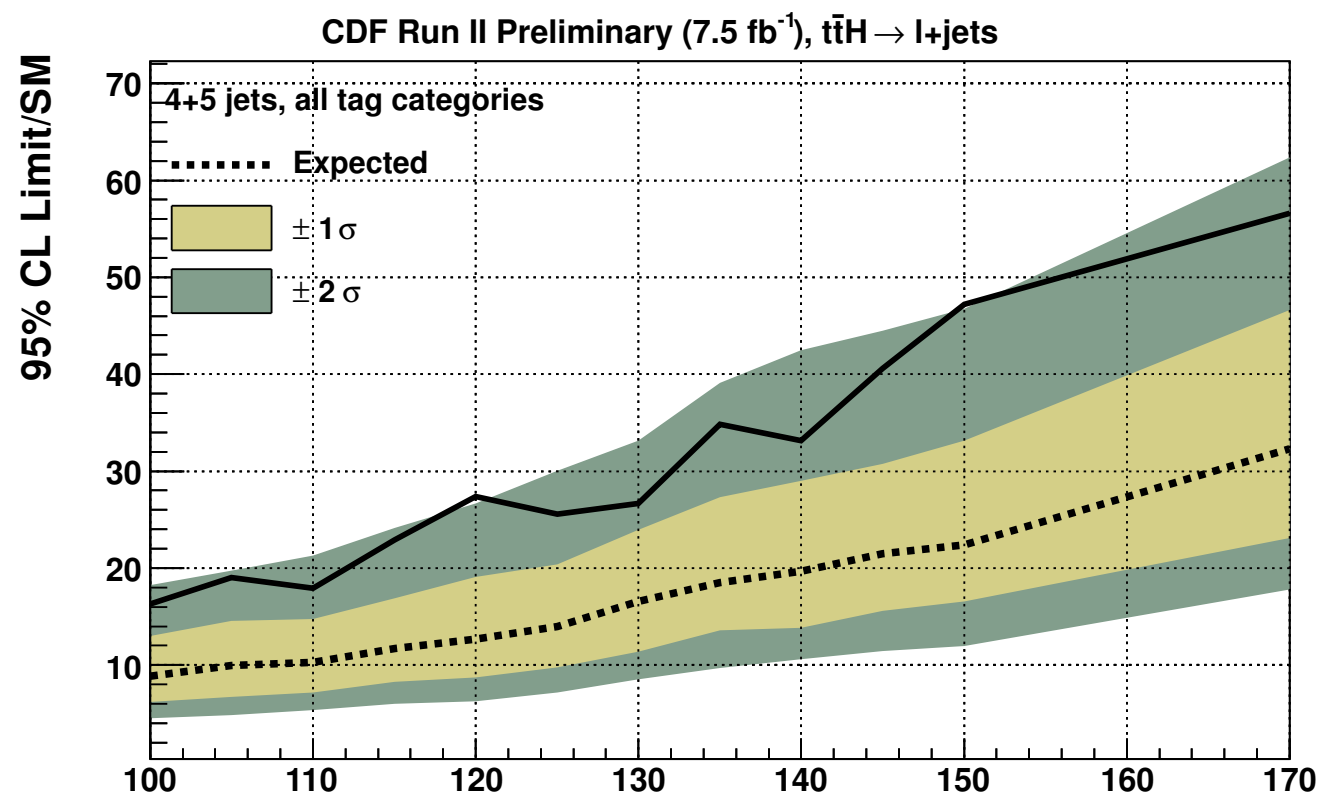

Figure 7.2: Expected and Observed $95 \%$ confidence level limits using all channels combined 


\begin{tabular}{ccccccc}
\hline$m_{H}$ & Obs & $-2 \sigma$ & $-1 \sigma$ & Exp & $+1 \sigma$ & $+2 \sigma$ \\
\hline 100 & $\mathbf{1 6 . 3}$ & 4.5 & 6.2 & $\mathbf{8 . 9}$ & 13.0 & 18.3 \\
105 & $\mathbf{1 9 . 0}$ & 4.8 & 6.7 & $\mathbf{1 0 . 0}$ & 14.5 & 19.7 \\
110 & $\mathbf{1 8 . 0}$ & 5.4 & 7.2 & $\mathbf{1 0 . 3}$ & 14.8 & 21.3 \\
115 & $\mathbf{2 2 . 9}$ & 6.0 & 8.3 & $\mathbf{1 1 . 7}$ & 16.9 & 24.1 \\
120 & $\mathbf{2 7 . 4}$ & 6.3 & 8.7 & $\mathbf{1 2 . 7}$ & 19.1 & 26.7 \\
125 & $\mathbf{2 5 . 6}$ & 7.2 & 9.7 & $\mathbf{1 4 . 0}$ & 20.4 & 30.1 \\
130 & $\mathbf{2 6 . 6}$ & 8.5 & 11.4 & $\mathbf{1 6 . 6}$ & 24.0 & 33.1 \\
135 & $\mathbf{3 4 . 9}$ & 9.7 & 13.6 & $\mathbf{1 8 . 5}$ & 27.3 & 39.1 \\
140 & $\mathbf{3 3 . 1}$ & 10.6 & 13.9 & $\mathbf{1 9 . 7}$ & 29.0 & 42.5 \\
145 & $\mathbf{4 0 . 6}$ & 11.5 & 15.6 & $\mathbf{2 1 . 5}$ & 30.7 & 44.5 \\
150 & $\mathbf{4 7 . 2}$ & 11.9 & 16.6 & $\mathbf{2 2 . 4}$ & 33.2 & 46.7 \\
170 & $\mathbf{5 6 . 6}$ & 17.8 & 23.1 & $\mathbf{3 2 . 3}$ & 46.6 & 62.4 \\
\hline \multicolumn{6}{c}{ CDF Run II Preliminary, $7.5 \mathrm{fb}^{-1}, t \bar{t} H \rightarrow \ell+$ jets }
\end{tabular}

Table 7.1: Expected and Observed 95\% confidence level limits using all channels combined

observe a limit of 27.4 times the Standard Model prediction, just outside of the upper $2 \sigma$ expected limit. 


\section{Chapter 8 CONCLUSION}

The Higgs boson is the last remaining particle that is predicted by the Standard Model but not yet observed in nature. The search for this particle is important because, if found, it will validate the Standard Model, and if excluded entirely, it will point the way forward to new physics. It is known that the electroweak symmetry must be spontaneously broken, and searches for the Higgs boson will elucidate the mechanism of this symmetry breaking.

Collider experiments at the LHC and the Tevatron provide the best chances to directly observe the Higgs boson. We have performed such a search for the Standard Model Higgs boson when produced in association with a top quark pair at the Collider Detector at Fermilab using $7.5 \mathrm{fb}^{-1}$ of proton-antiproton collision data at $\sqrt{s}=1.96 \mathrm{TeV}$. This search exploits the unique final state of two $W$ bosons, two $b$ quarks, and the Higgs decay products in order to substantially reduce the background to Higgs production.

We also exploit the unique kinematic properties of $t \bar{t} H$ events compared to $t \bar{t}$ events to train ensembles of neural networks to discriminate Higgs events from background events. These discriminants provide much better classification power than any single kinematic variable. This separation gives us orthogonal regions with a wide range of signal purity and background content, allowing us to constrain the rate of the backgrounds in the most signal-like regions and significantly improving the sensitivity of the analysis.

We use a Bayesian technique to establish $95 \%$ confidence level upper bounds on the $t \bar{t} H$ production cross section. We account for systematic uncertainties in our model by treating them as nuisance parameters and integrating them out using a Monte Carlo numerical 


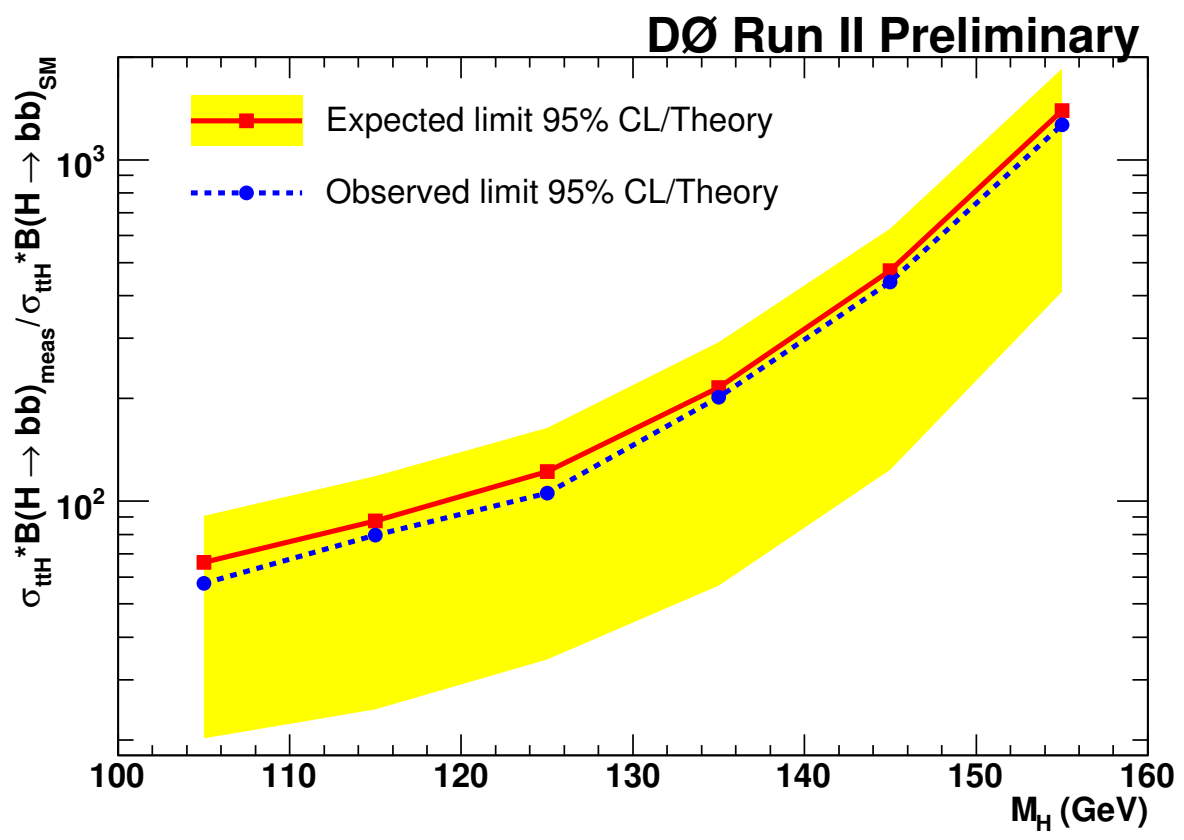

Figure 8.1: DØ $2009 t \bar{t} H$ search

integration technique. Based only on our background and signal model, we expect to set a limit of 12.7 times the Standard Model predicted cross section at $m_{H}=120 \mathrm{GeV} / c^{2}$. Upon examining the data, we observe a limit of 27.4 times the Standard Model predicted cross section.

\subsection{Other searches for $t \bar{t} H$}

In 2008, CDF performed a search for $t \bar{t} H$ in the lepton plus jets channel, restricting the Higgs decay to $b \bar{b}$. That search used $300 \mathrm{pb}^{-1}$ of data, and was able to set a limit of 169 times the Standard Model predicted cross section at $m_{H}=120 \mathrm{GeV} / c^{2}[49]$. In 2009, DØ performed a similar search using $2.1 \mathrm{fb}^{-1}$, and set a limit of 45 times the Standard Model prediction at $m_{H}=120 \mathrm{GeV} / c^{2}[50]$.

CDF has also performed a search for a Standard Model Higgs boson in the $t \bar{t} H$ production

mode in the zero-lepton final state using $5.7 \mathrm{fb}^{-1}$. There are two principal contributors to this analysis: low- $\mathbb{E}_{T}$ and high- $\mathbb{E}_{T}$ events. Low- $\mathbb{E}_{T}$ events are principally those in which all 


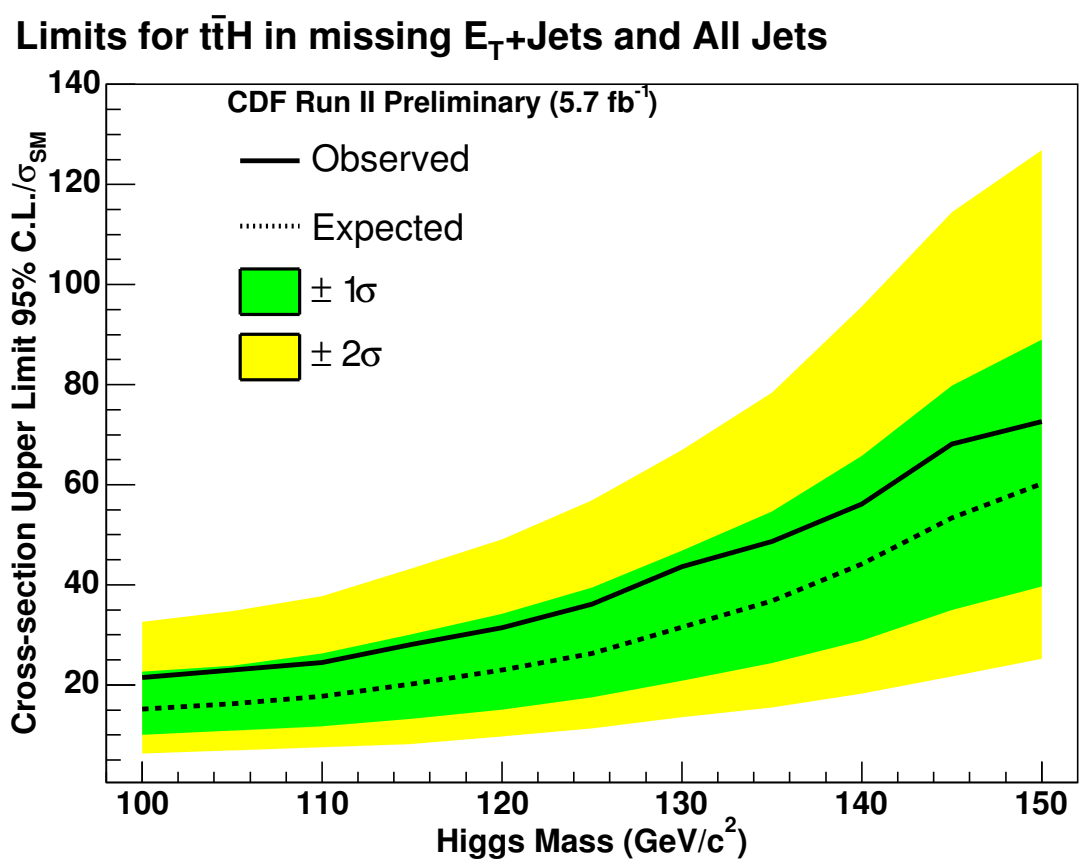

Figure 8.2: Limits on $t \bar{t} H$ production at CDF in the zero lepton final state

of the $W$ bosons decayed hadronically. This mode is dominated by the QCD background, but we are able to obtain useful sensitivity by training neural networks to discriminate all-hadronic $t \bar{t}$ from QCD, reducing the QCD background significantly. High- $\mathbb{E}_{T}$ events are events in which one of the $W$ bosons decayed leptonically, but we failed to reconstruct the lepton. This can occur due to a variety of effects, including geometric acceptance and overlap of the lepton's signal with a jet. Additionally, because the lepton plus jets $t \bar{t} H$ analysis only considers electrons and muons, the zero lepton channel gains substantial acceptance from events in which one of the $W$ bosons decayed to a tau and tau neutrino. This channel is also dominated by the QCD background, but the addition of the missing transverse energy both reduces the background and gives us a handle to help discriminate the $t \bar{t} H$ signal from the $t \bar{t}$ and QCD backgrounds.

This search also trained discriminants and used mclimit to establish $95 \%$ confidence level upper bounds on the $t \bar{t} H$ cross section, which can be seen in figure 8.2 . At $m_{H}=120 \mathrm{GeV} / c^{2}$, we set a limit of 31.4 times the Standard Model prediction. 


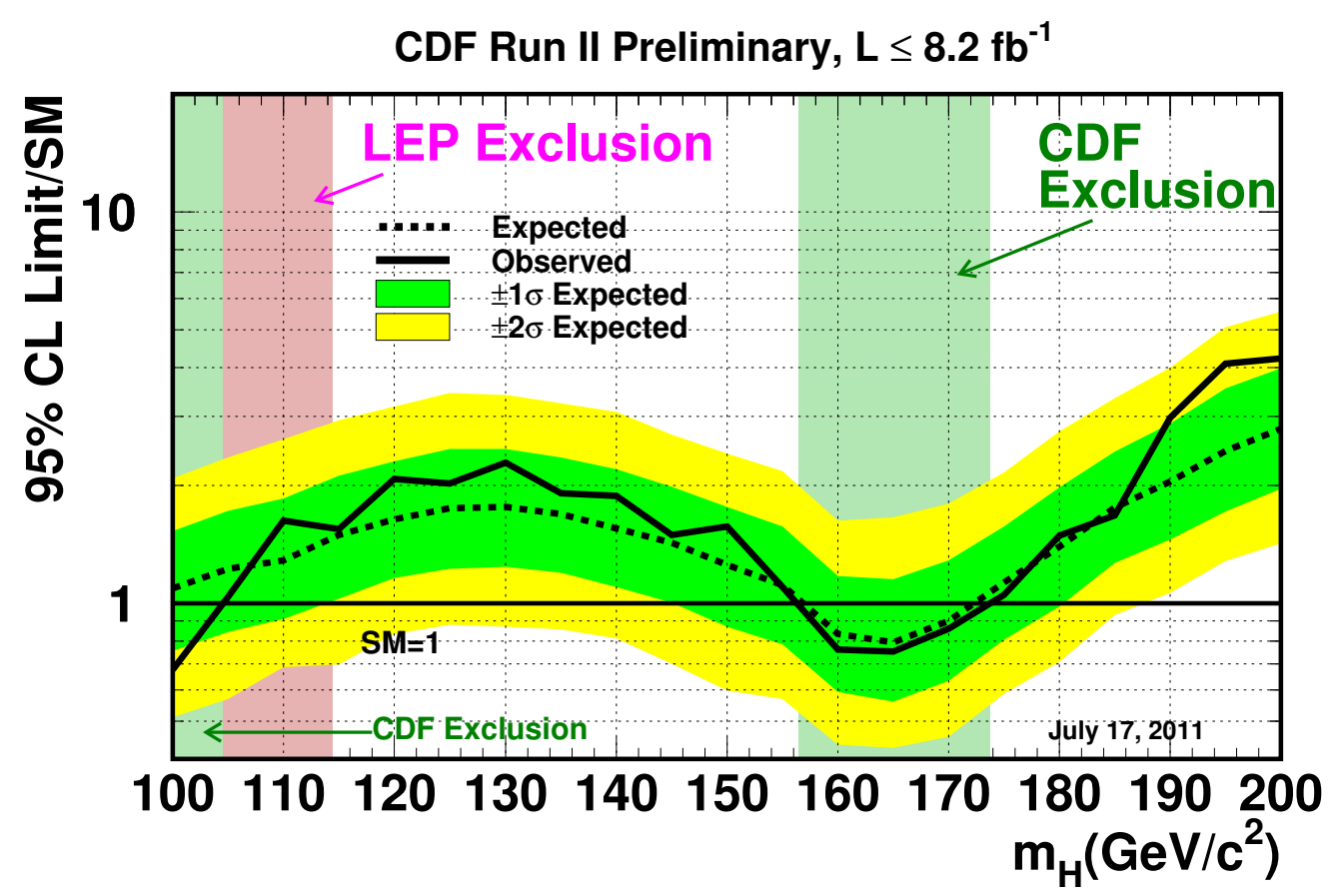

Figure 8.3: CDF combined limits on Higgs production

\subsection{Combined Higgs searches}

We also combine this result with other searches for the Higgs boson, at CDF and at DØ. Using this combination, CDF alone is able to exclude the existence of a Standard Model Higgs boson with $156.5<m_{H}<173.7 \mathrm{GeV} / c^{2}$ or $100<m_{H}<104.5 \mathrm{GeV} / c^{2}$. The CDF result, as can be seen in figure 8.4, is dominated at high mass by the $H \rightarrow W W$ analysis, and at low mass by the vector boson associated production channels. These analyses are accompanied by a host of secondary analyses, of which $t \bar{t} H$ is the fourth most sensitive.

In addition to CDF, D $\varnothing$ also performs searches for the Higgs boson. The set of analyses that they perform is similar to CDF's, although $\mathrm{D} \varnothing$ does not search for the Higgs boson in the $t \bar{t} H$ channel. Their combined results are shown in figure 8.5, and they are able to exclude a Standard Model Higgs boson with $161<m_{H}<170 \mathrm{GeV} / c^{2}$ or $100<m_{H}<105 \mathrm{GeV} / c^{2}$.

By combining both of the experiments at the Tevatron, CDF and DØ, we can expand the excluded regions to exclude the existence of a Standard Model Higgs boson with $156<m_{H}<177 \mathrm{GeV} / c^{2}$ or $100<m_{H}<108 \mathrm{GeV} / c^{2}[47]$. 


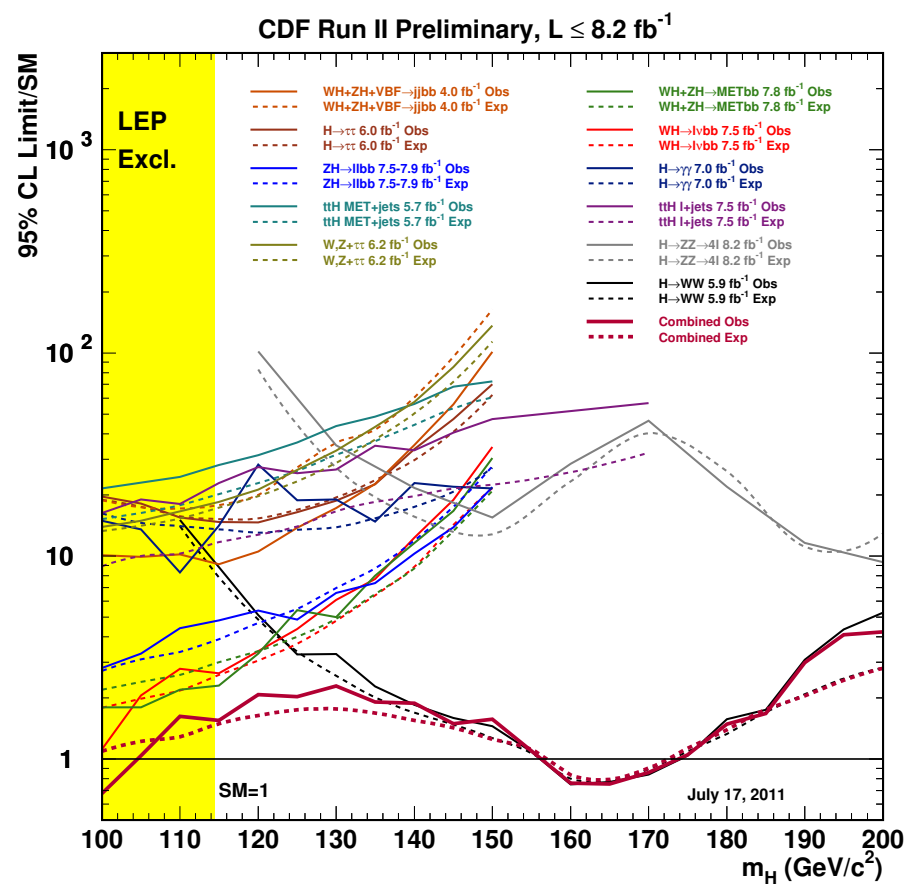

Figure 8.4: Contributions of individual analyses to the CDF combination

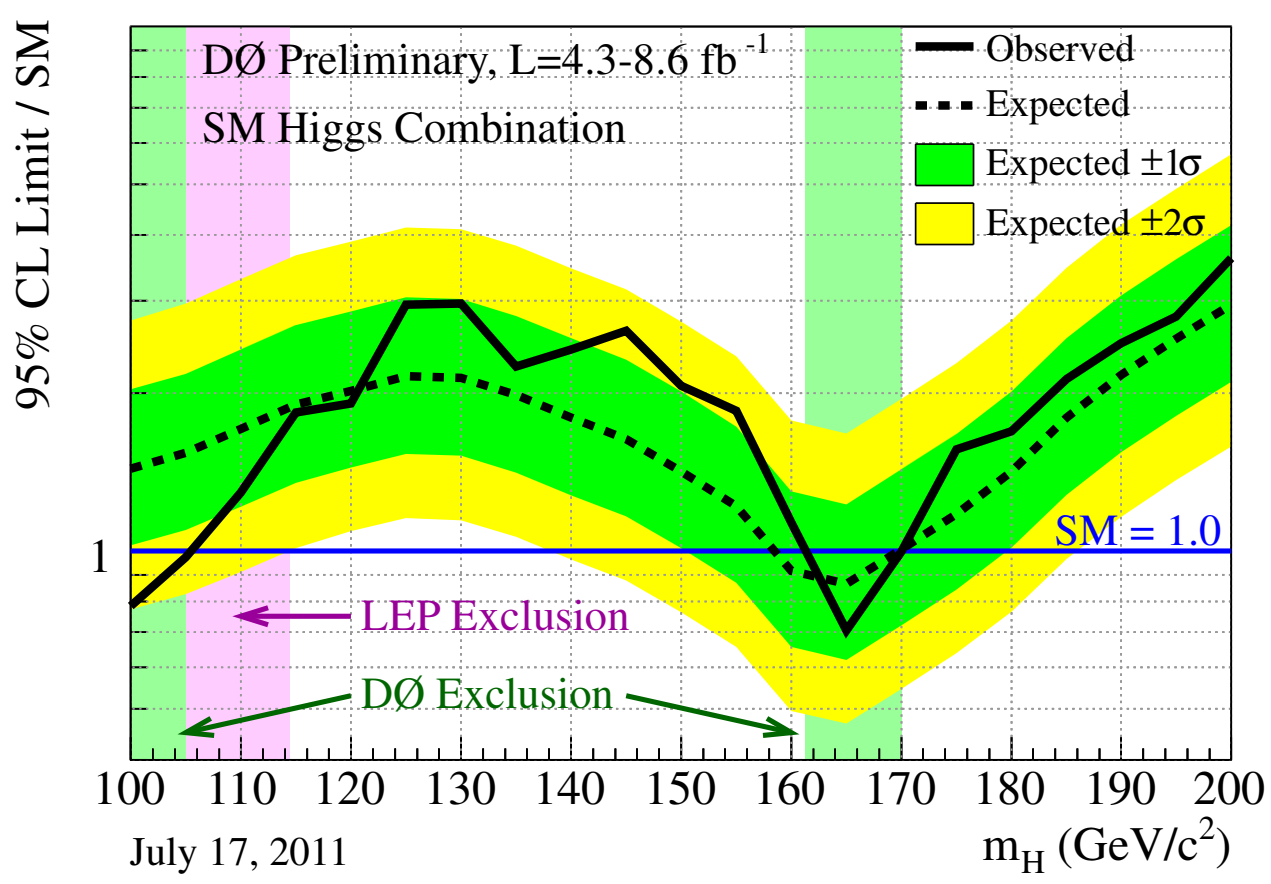

Figure 8.5: DØ combined limits on Higgs production 


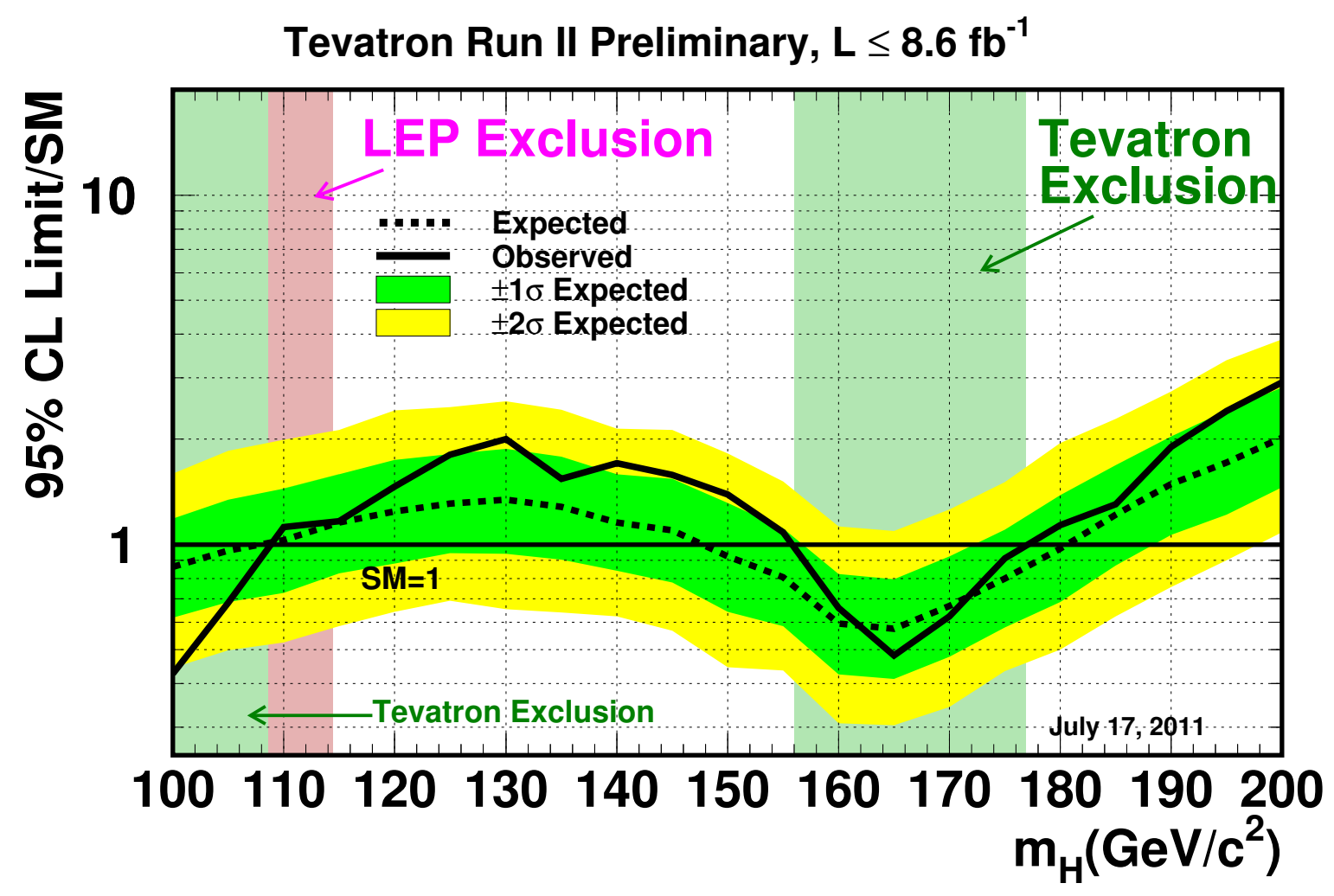

Figure 8.6: Tevatron (CDF and DØ) combined limits on Higgs production 


\section{BIBLIOGRAPHY}

[1] L.Reina, S.Dawson, and D.Wackeroth, QCD corrections to Associated t-tbar-H production at the Tevatron, Phys. Rev. D 65 (2002).

[2] W. Beenakker et al., Higgs Radiation off Top Quarks at the Tevatron and the LHC, Phys. Rev. Lett. 87 (2001).

[3] Particle Data Group, K. Nakamura et al., Review of Particle Physics, 37 (2010).

[4] J. C. Maxwell, A dynamical theory of the electromagnetic field, Philos. T. R. Soc. Lond. 155, 459 (1865).

[5] A. A. Michelson and E. W. Morley, On the relative motion of the earth and the luminiferous ether, Am. J. Sci. 34, 333 (1887).

[6] A. Einstein, Zur Elektrodynamik bewegter Körper, Annalen der Physik 322, 891 (1905).

[7] D. J. Griffiths, Introduction to Particle Physics, 2nd ed. (WILEY-VCH, 2008).

[8] H. A. Bethe and R. Jackiw, Intermediate Quantum Mechanics, Third ed. (AddisonWesley, 1997).

[9] R. Brandelik et al., Evidence for planar events in $\mathrm{e}^{+} \mathrm{e}^{-}$annihilation at high energies, Physics Letters B 86, 243 (1979).

[10] L. Meitner, Über die Entstehung der $\beta$-Strahl-Spektren radioaktiver Substanzen, Zeitschrift fur Physik 9, 131 (1922).

[11] K. Riesselmann, logbook: neutrino invention, Symmetry Magazine 03 (2006).

[12] T.-P. Cheng and L.-F. Li, Gauge Theory of Elementary Particle Physics (Clarendon Press Oxford, 1984).

[13] S. Glashow, Partial Symmetries of the Weak Interactions, Nucl. Phys. 22, 579 (1961).

[14] S. Weinberg, A Model of Leptons, Phys. Rev. Lett. 19, 1264 (1967).

[15] A. Salam, Elementary particle physics: Relativistic groups and analyticity, Eighth Nobel Symposium, 1968. 
[16] UA1, G. Arnison et al., Experimental observation of isolated large transverse energy electrons with associated missing energy at $\mathrm{s}^{* *}(1 / 2)=540-\mathrm{GeV}$, Phys. Lett. B122, 103 (1983).

[17] UA1, G. Arnison et al., Experimental observation of lepton pairs of invariant mass around $95-\mathrm{GeV} / \mathrm{c}^{* *} 2$ at the CERN SPS collider, Phys. Lett. B126, 398 (1983).

[18] CDF, F. Abe et al., Observation of top quark production in $p \bar{p}$ collisions, Phys. Rev. Lett. 74 (1995).

[19] J. C. L. Cowan, F. Reines, F. B. Harrison, H. W. Kruse, and A. D. McGuire, Detection of the Free Neutrino: a Confirmation, Science 124, 103 (1956).

[20] F. Englert and R. Brout, Broken Symmetry and the Mass of Gauge Vector Mesons, Physical Review Letters 13, 321 (1964).

[21] P. W. Higgs, Broken Symmetries and the Masses of Gauge Bosons, Phys. Rev. Lett. 13, 508 (1964).

[22] G. S. Guralnik, C. R. Hagen, and T. W. Kibble, Global Conservation Laws and Massless Particles, Physical Review Letters 13, 585 (1964).

[23] Gfitter, J. Haller et al., Revisiting the Global Electroweak Fit of the Standard Model and Beyond with Gfitter, Eur. Phys. J. C 60, 543 (2009).

[24] The LEP Working Group for Higgs Boson Searches, G. Abbiendi et al., Search for the Standard Model Higgs Boson at LEP, Phys. Lett .B 565, 61 (2003).

[25] C. Gattutso et al., Accelerator concepts, V3.6, 2010.

[26] B. Worthel, Booster rookie book, V4.1, 2009.

[27] Minerva neutrino experiment, http://minerva.fnal.gov/.

[28] Minos experiment and numi beam home page, http://www-numi.fnal.gov/.

[29] T. Asher, Main injector rookie book, V1.1, 2003.

[30] J. Morgan et al., Antiproton source rookie book, V2.1, 2009.

[31] D. Crawford, Tevatron rookie book, V2.3, 2009.

[32] CDF, The CDF II Detector Technical Design Report, 1996, FERMILAB-Pub-96/390-E.

[33] CDF, A. Sill, SVX-II: CDF Run II Silicon Tracking Projects, Nucl. Instrum. Meth. A447 (2000).

[34] CDF, C. Hill, L00: Operational Experience and Performance of the CDFII Silicon Detector, Nucl. Instrum. Meth. A530 (2004).

[35] CDF, A. Abulencia et al., Measurements of inclusive $W$ and $Z$ cross sections in $p \bar{p}$ collisions at $\sqrt{s}=1.96 \mathrm{TeV}$, J. Phys.G Nucl. Part. Phys. 34 (2007). 
[36] CDF, A. Abulencia et al., Measurement of the $t \bar{t}$ production cross section in $p \bar{p}$ collisions at $\sqrt{s}=1.96 \mathrm{TeV}$ using lepton + jets events with jet probability $b$-tagging, Phys. Rev. D 74 (2006).

[37] CDF, F. Abe et al., Topology of three-jet events in $\bar{p} p$ collisions at $\sqrt{s}=1.8 \mathrm{TeV}$, Phys. Rev. D 45 (1992).

[38] CDF, D. Acosta et al., Measurement of the $t \bar{t}$ production cross section in $p \bar{p}$ collisions at $\sqrt{s}=1.96 \mathrm{TeV}$ using lepton + jets events with secondary vertex $b$-tagging, Phys. Rev. D 71 (2005).

[39] S. M. Torbjörn Sjöstrand and P. Skands, PYTHIA 6.4 physics and manual, JHEP 05 (2006).

[40] J. Alwall et al., MadGraph/MadEvent v4: The New Web Generation, JHEP 0709 (2007).

[41] M. Mangano, M. Moretti, F. Piccinini, R. Pittau, and A. Polosa, ALPGEN, a generator for hard multiparton processes in hadronic collisions, JHEP 0307 (2003).

[42] GEANT 4, S. Agostinelli et al., GEANT 4-A Simulation Toolkit, Nucl. Instrum. Meth. A503, 950 (2003).

[43] CDF Collaboration, D. Acosta et al., Measurement of the $t \bar{t}$ production cross section in $p \bar{p}$ collisions at $\sqrt{s}=1.96 \mathrm{TeV}$ using lepton + jets events with secondary vertex $b$ -tagging, Phys. Rev. D 71, 052003 (2005).

[44] R. A. Jacobs, Methods For Combining Experts' Probability Assessments, Neural Computation 7, 867 (1995).

[45] G. Cybenko, Approximation by superpositions of a sigmoidal function, Mathematics of Control, Signals, and Systems (MCSS) 2, 303 (1989), 10.1007/BF02551274.

[46] C. G. BROYDEN, The convergence of a class of double-rank minimization algorithms 1. general considerations, IMA Journal of Applied Mathematics 6, 76 (1970), http://imamat.oxfordjournals.org/content/6/1/76.full.pdf + html.

[47] T. CDF, D. Collaborations, t. T. N. Phenomena, and H. W. Group, Combined CDF and D0 Upper Limits on Standard Model Higgs Boson Production with up to $8.6 \mathrm{fb}-1$ of Data, (2011), 1107.5518.

[48] CDF, J. Heinrich, Bayesian limit software: multi-channel with correlated backgrounds and efficiences, (2005), CDF/MEMO/STATISTICS/PUBLIC/7587.

[49] S. Lai, Search for Standard Model Higgs Boson Produced with ttbar in 1.96 TeV ppbar Collisions, PhD thesis, University of Toronto, 2008, CDF/THESIS/EXOTIC/PUBLIC/9508.

[50] $\mathrm{D} \varnothing$ Search for the standard model higgs boson in the $t \bar{t} h \rightarrow t \bar{t} b \bar{b}$ channel, 2009, DØ note 5739-CONF. 


\section{Appendix A VALIDATION}

These plots demonstrate that, in all the channels of our analysis, the variables that are inputs to the discriminants, as well as the discriminant outputs, are sufficiently well-modeled.

\section{A.1 Discriminant inputs}

\section{A.1.1 4 jets, $2 b$ tags}
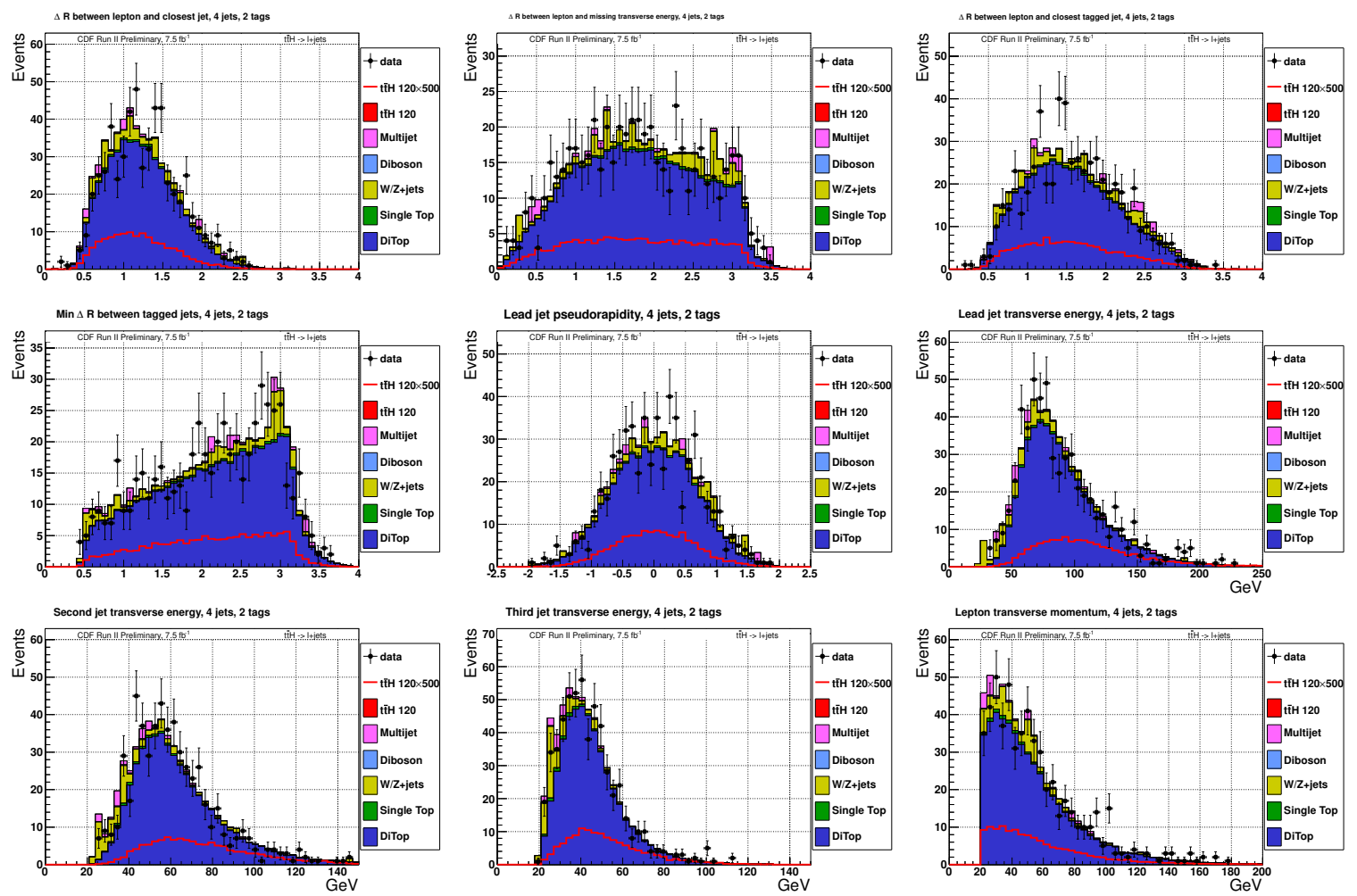

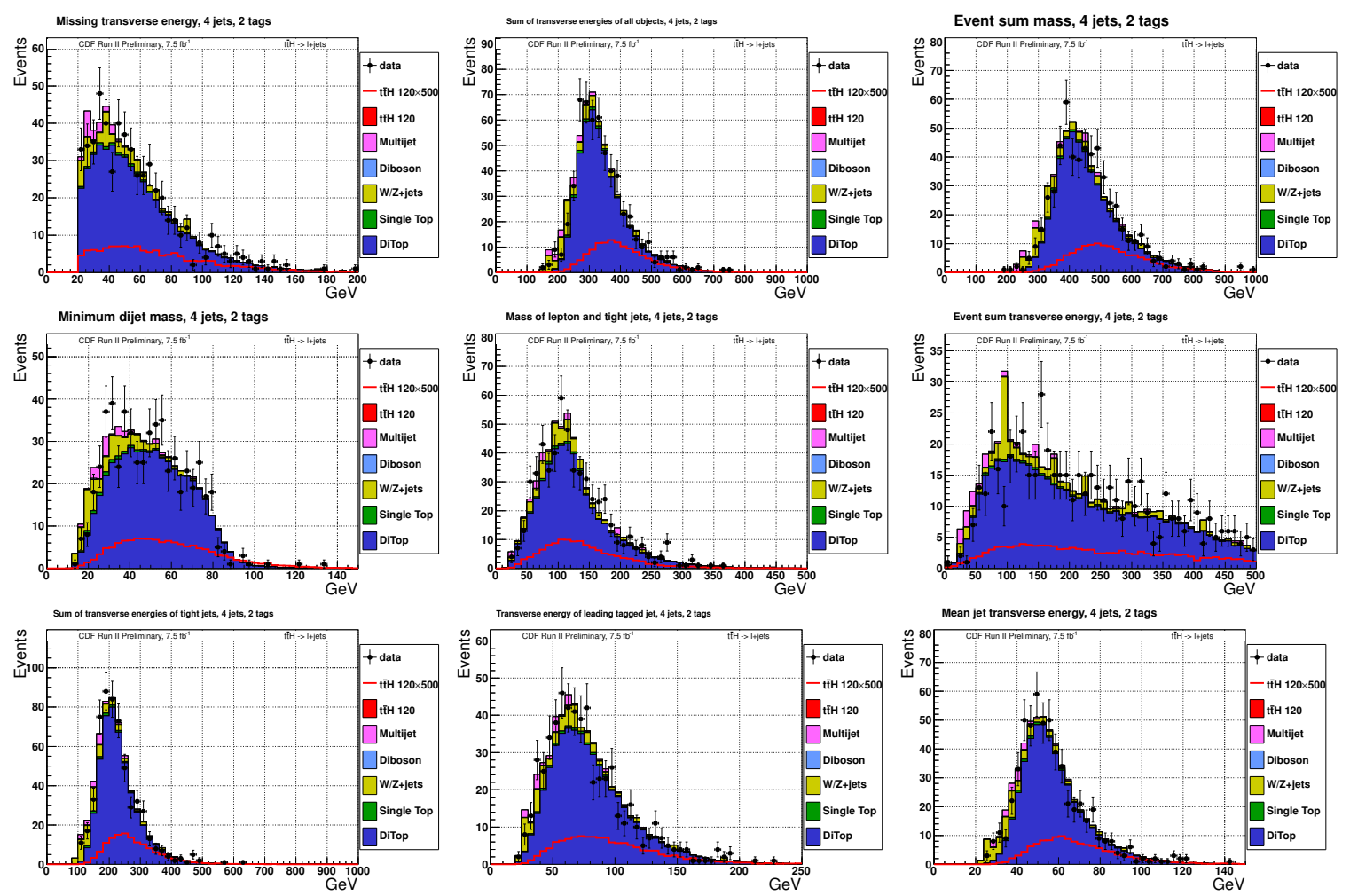

\section{A.1.2 4 jets, $\geq 3 b$ tags}
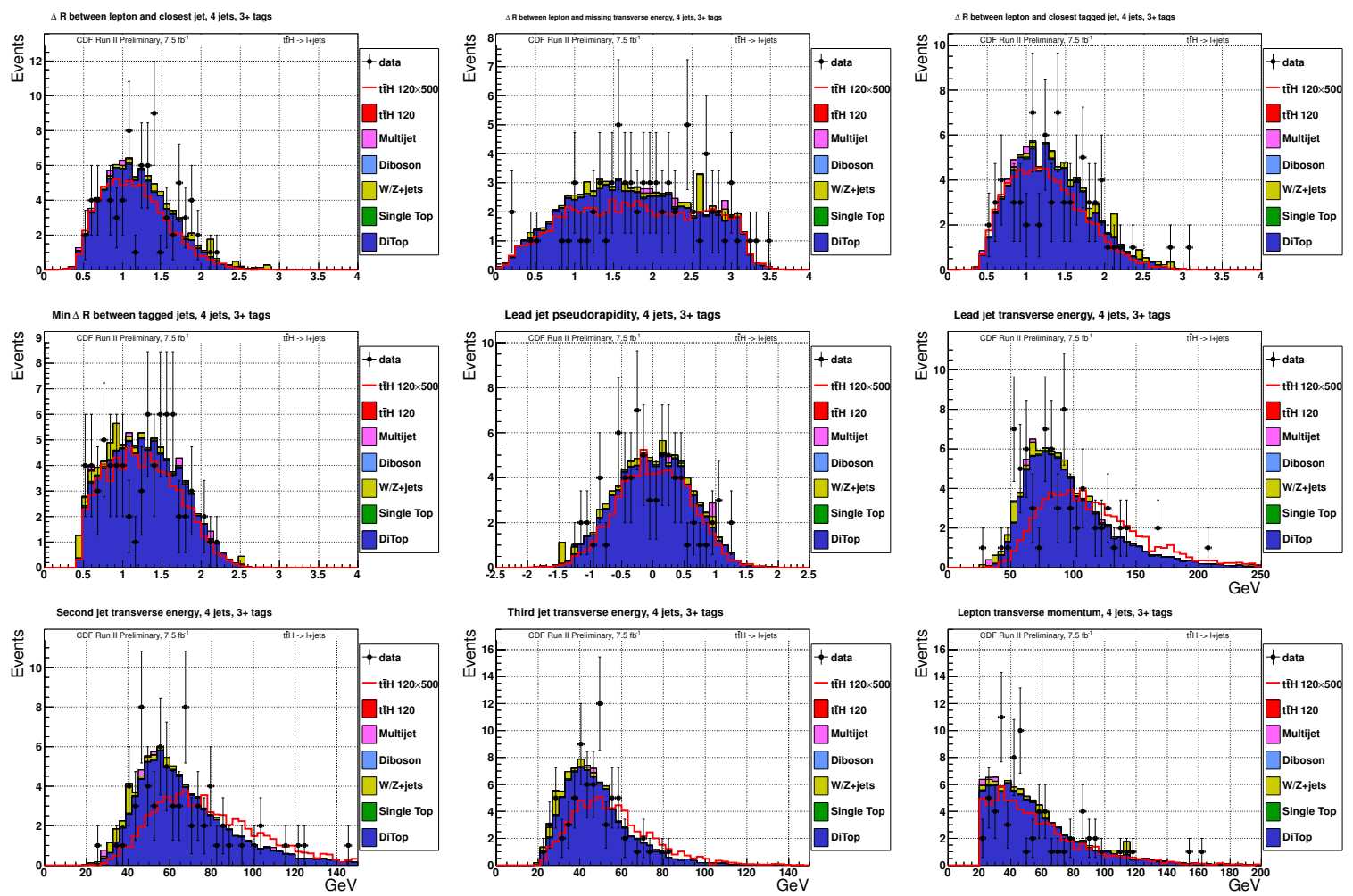

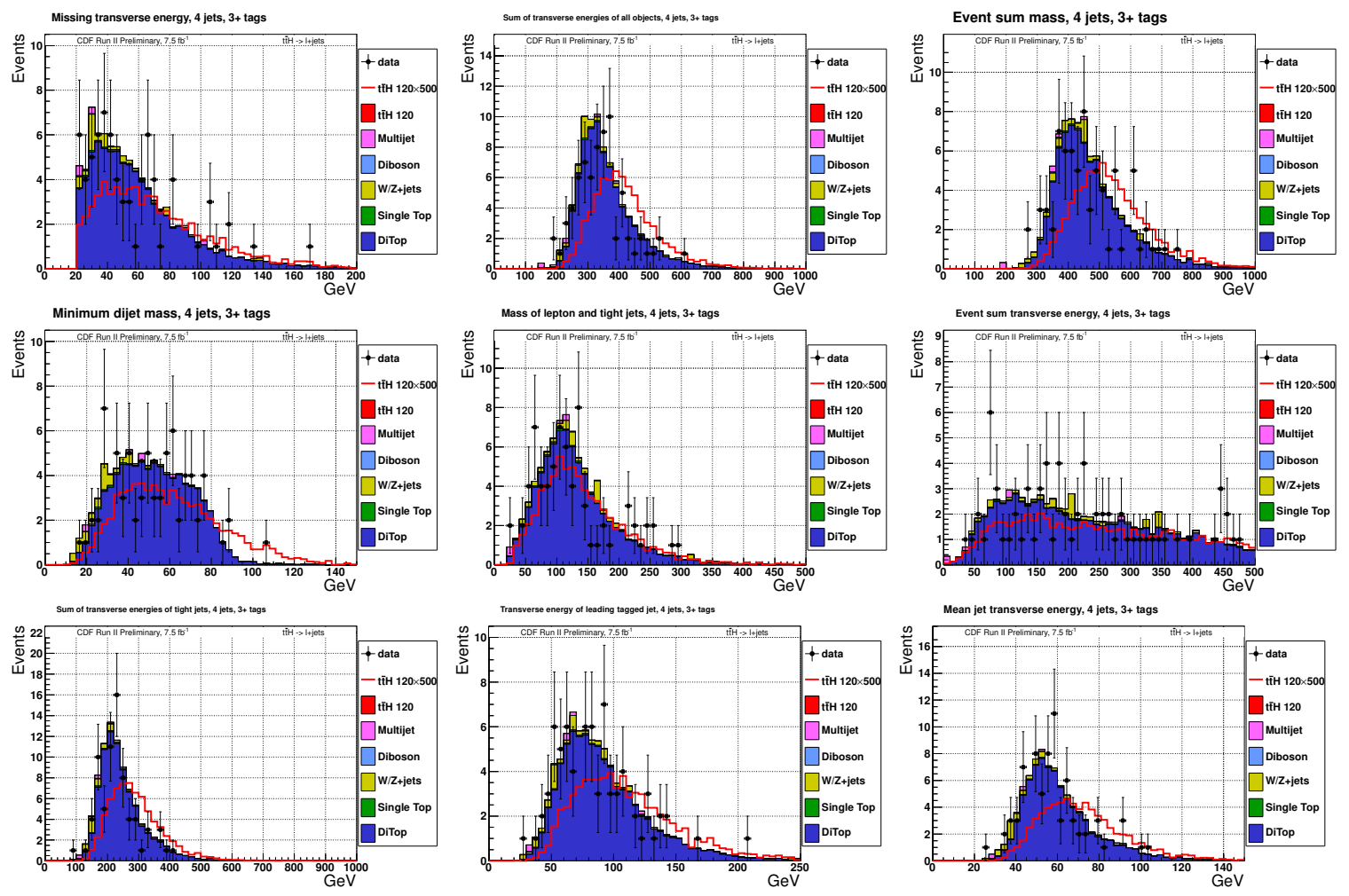

\section{A.1.3 $\geq 5$ jets, $2 b$ tags}
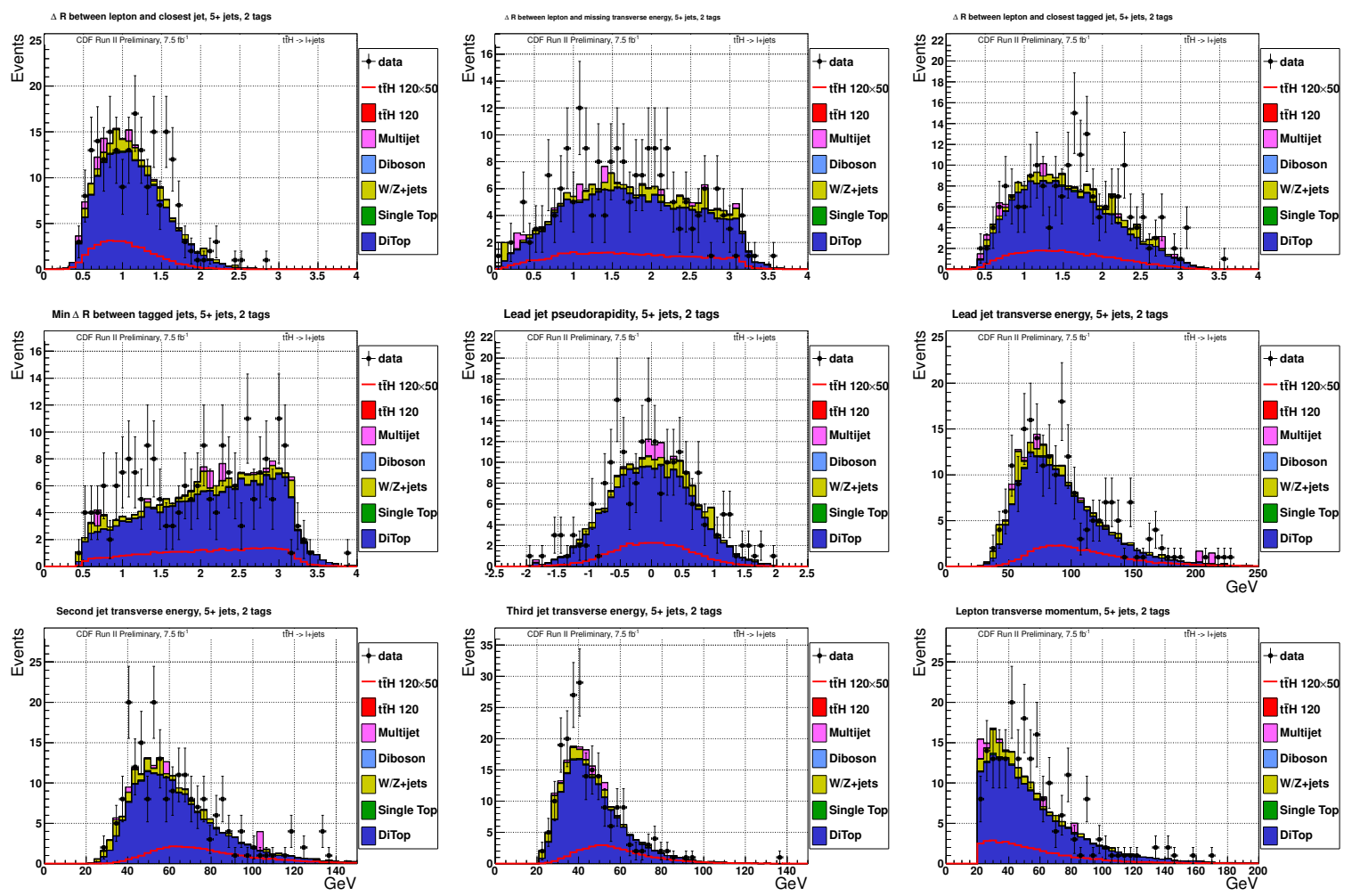

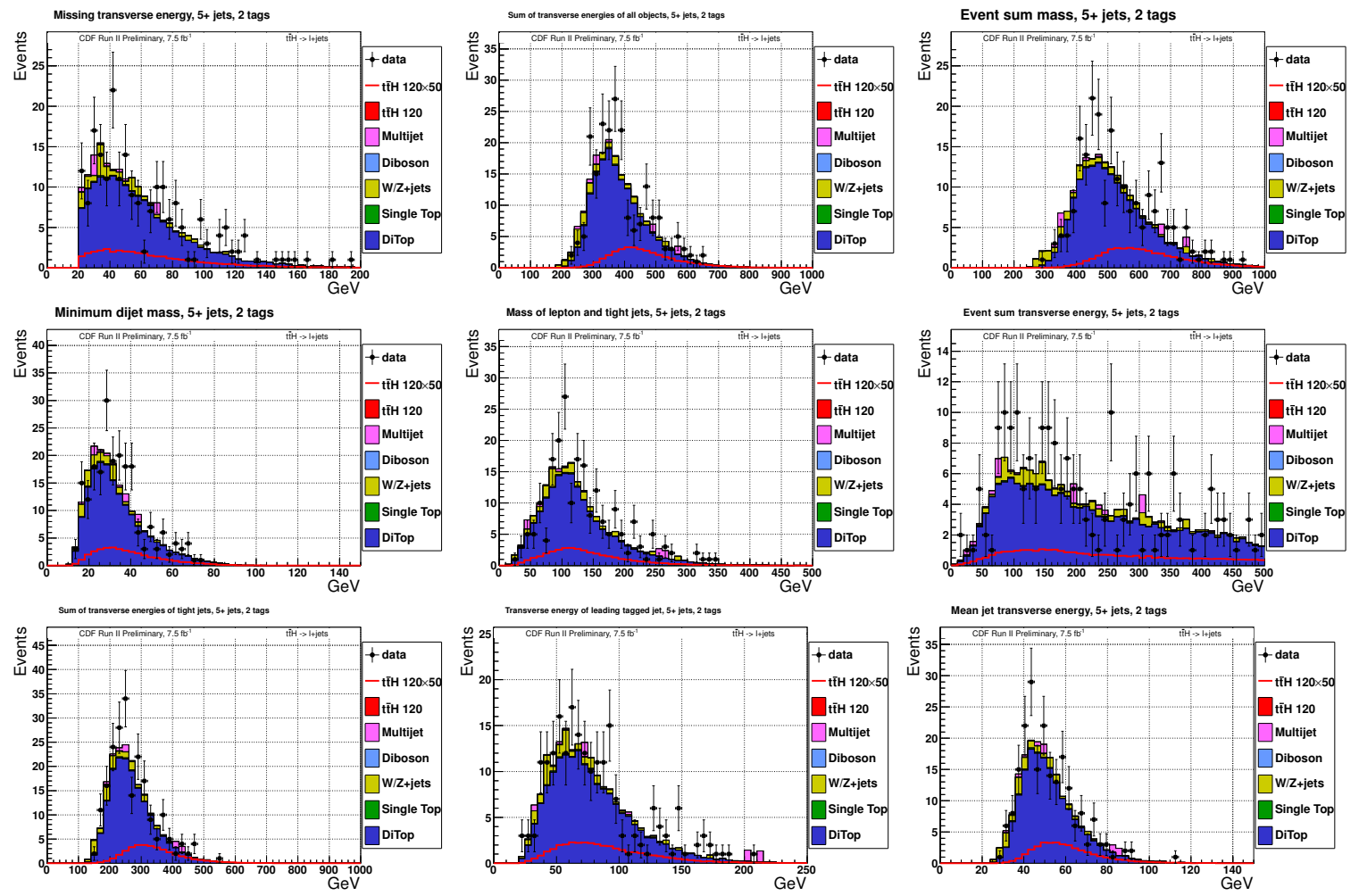

\section{A.1.4 $\geq 5$ jets, $\geq 3 b$ tags}
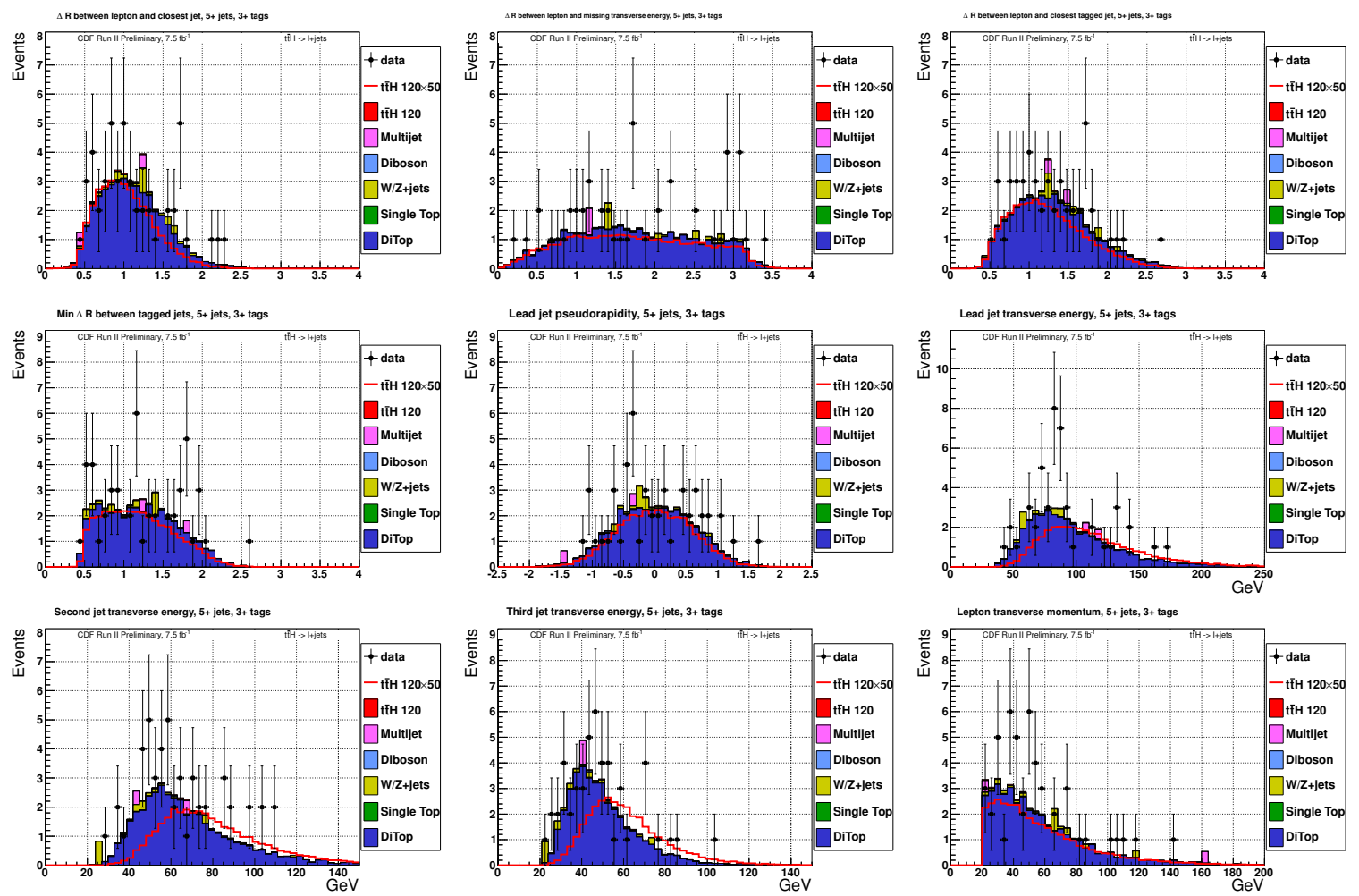

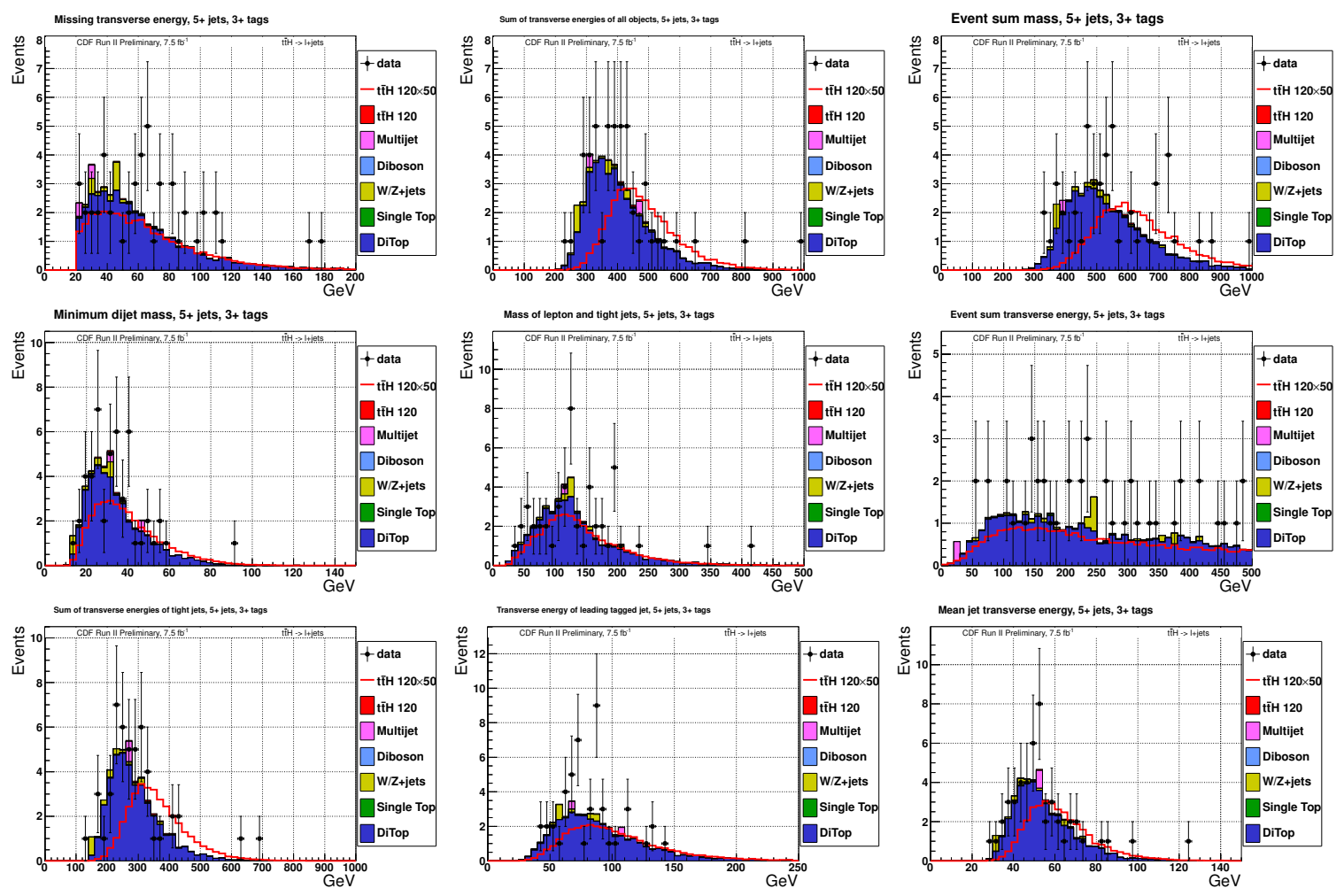

\section{A.2 Discriminant outputs}

$$
m_{H}=100 \mathrm{GeV} / c^{2}
$$
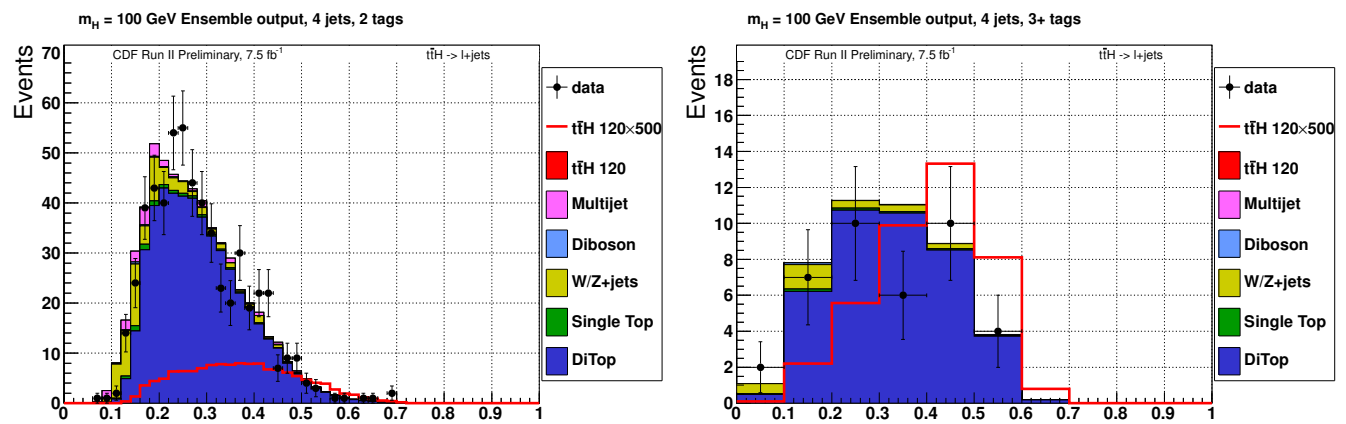

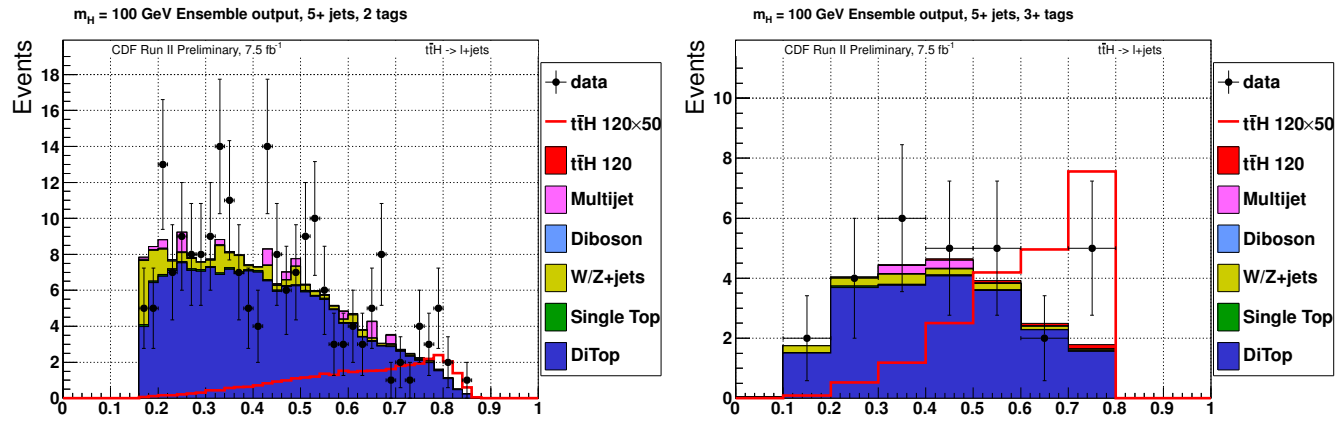

$$
m_{H}=105 \mathrm{GeV} / c^{2}
$$
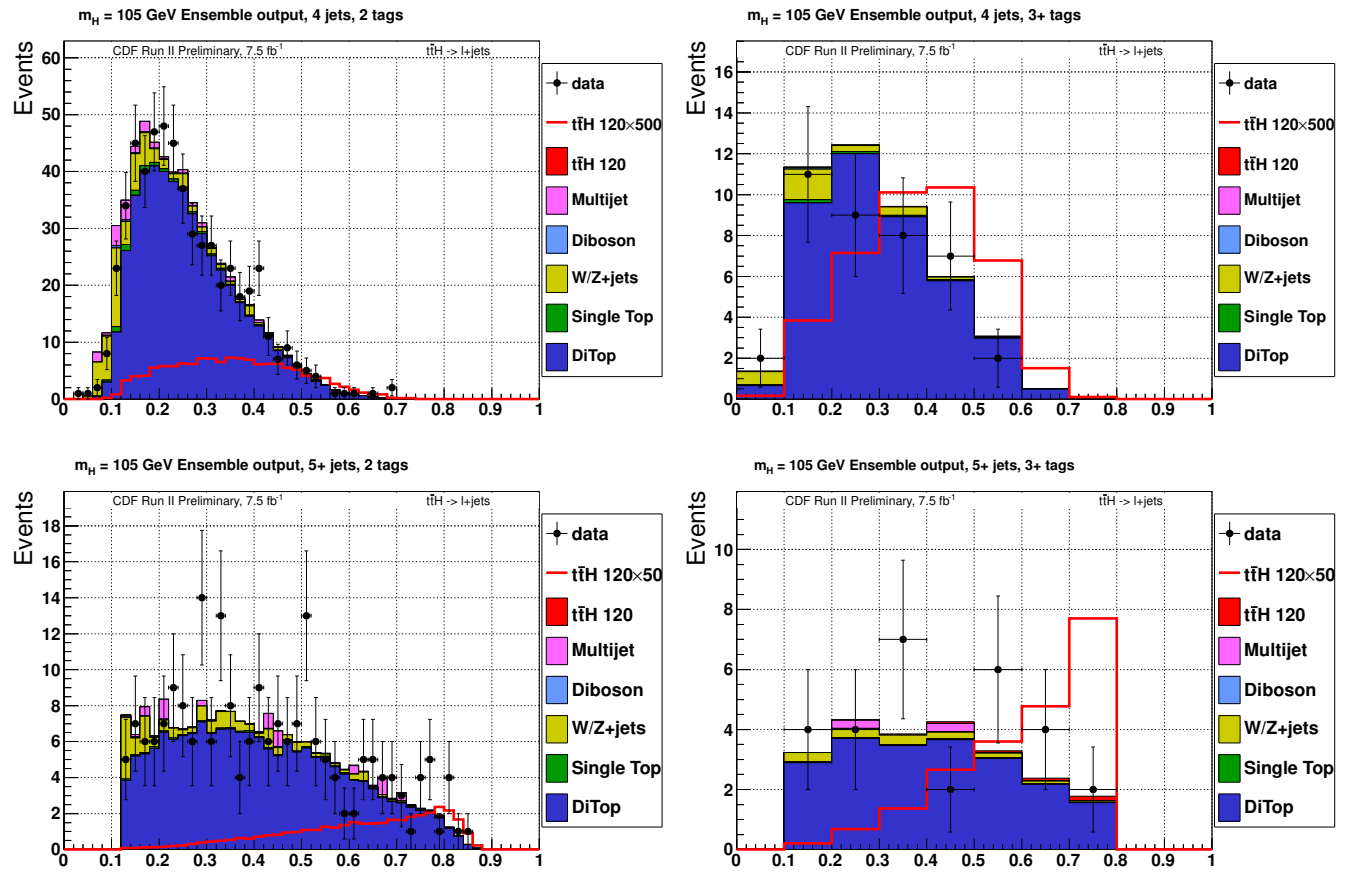

$$
m_{H}=110 \mathrm{GeV} / c^{2}
$$
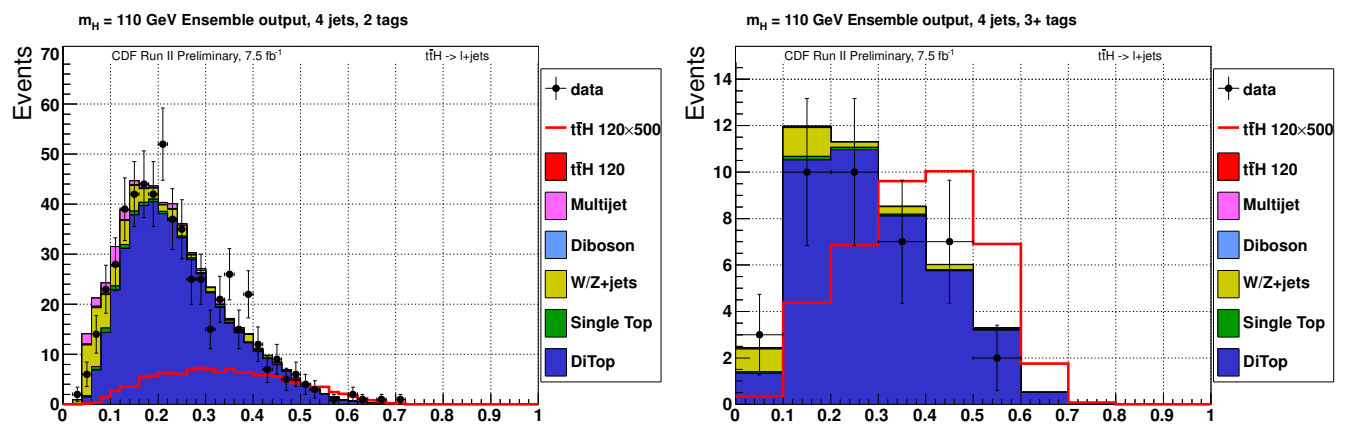

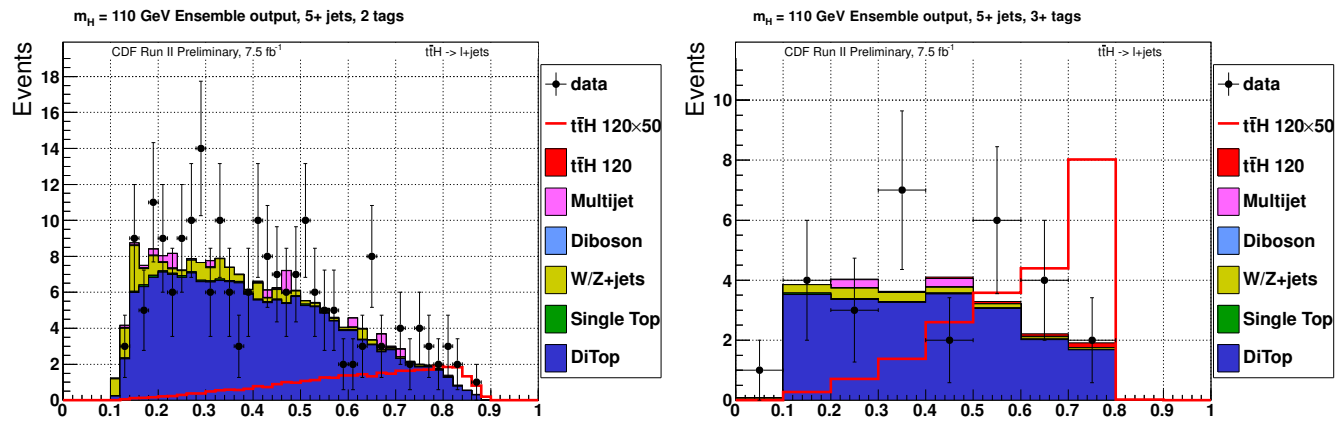

$$
m_{H}=115 \mathrm{GeV} / c^{2}
$$
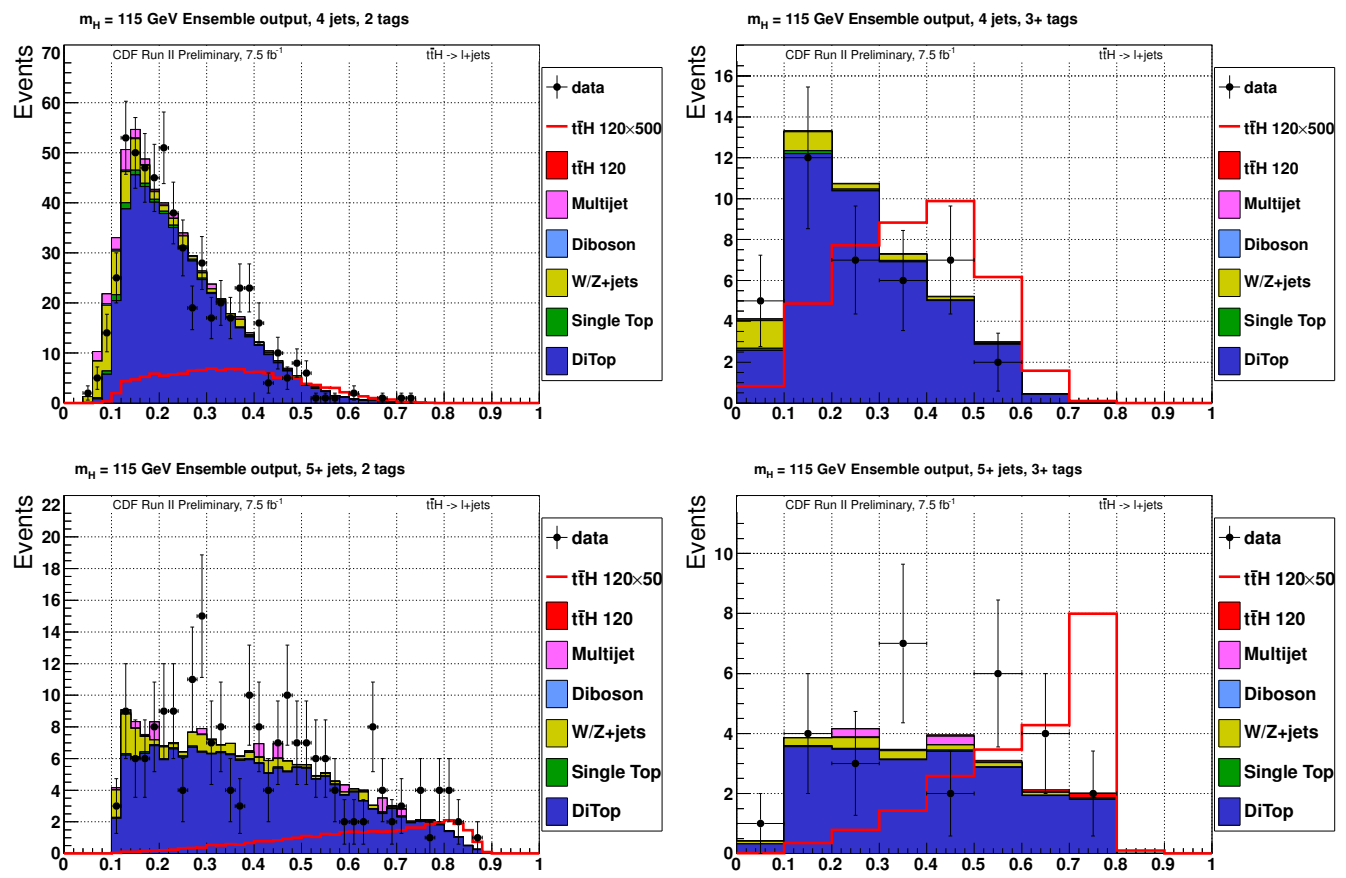

$$
m_{H}=120 \mathrm{GeV} / c^{2}
$$
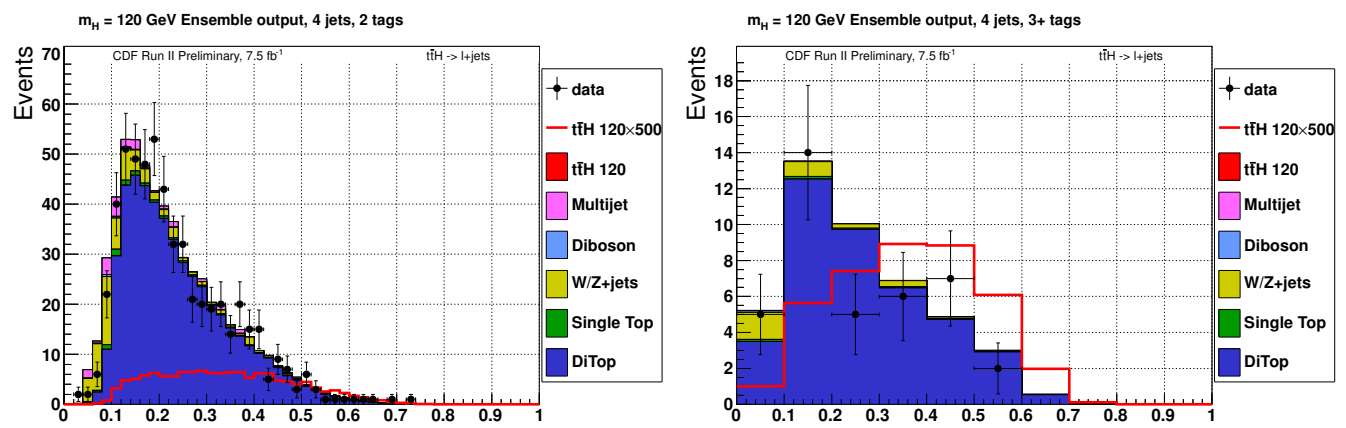

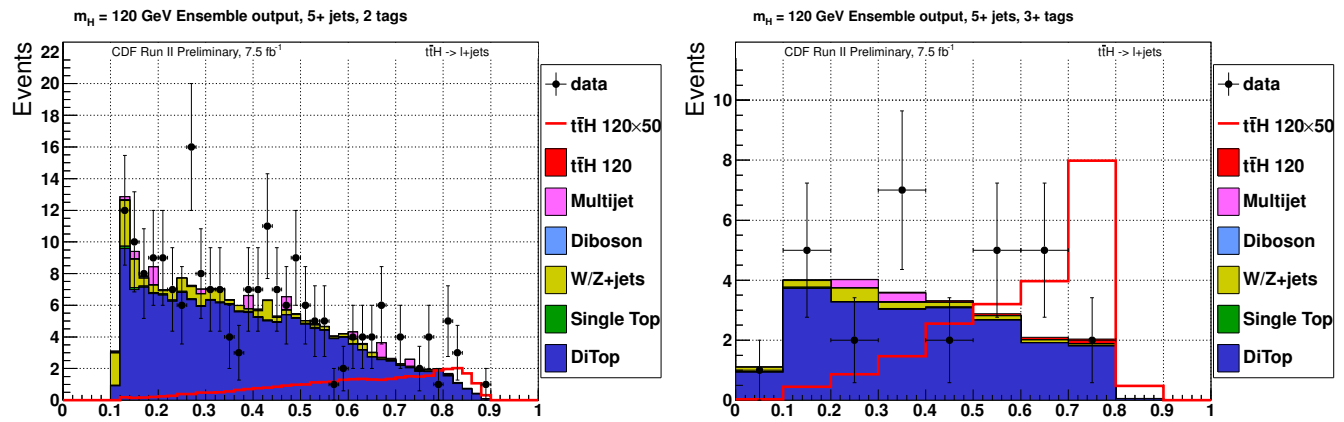

$$
m_{H}=125 \mathrm{GeV} / c^{2}
$$
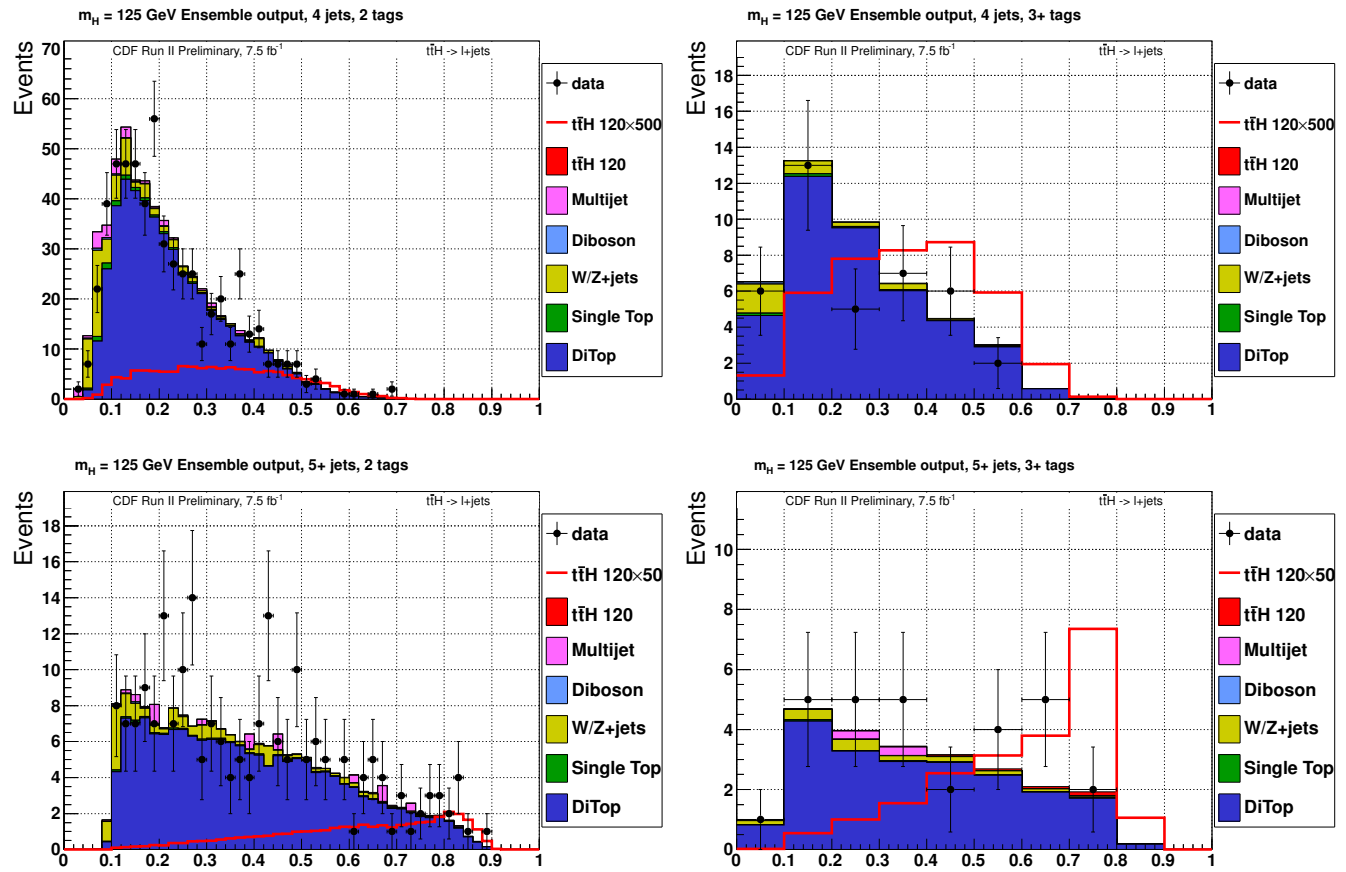

$$
m_{H}=130 \mathrm{GeV} / c^{2}
$$
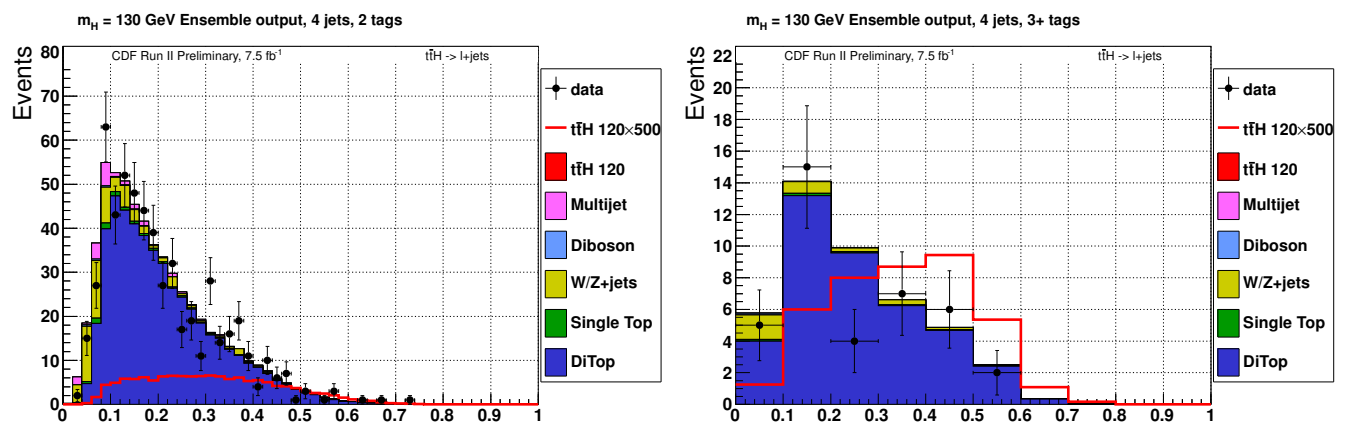

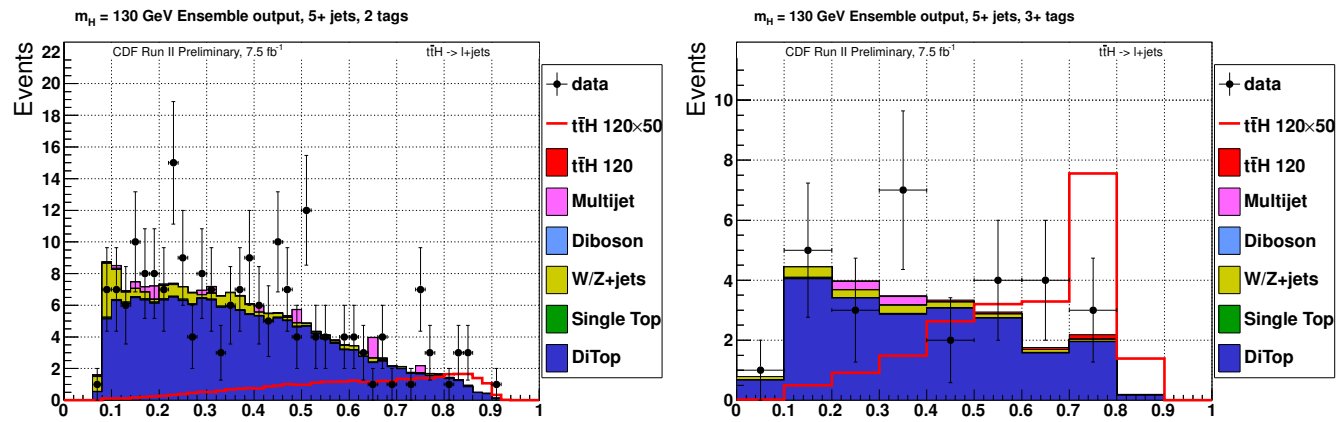

$$
m_{H}=135 \mathrm{GeV} / c^{2}
$$
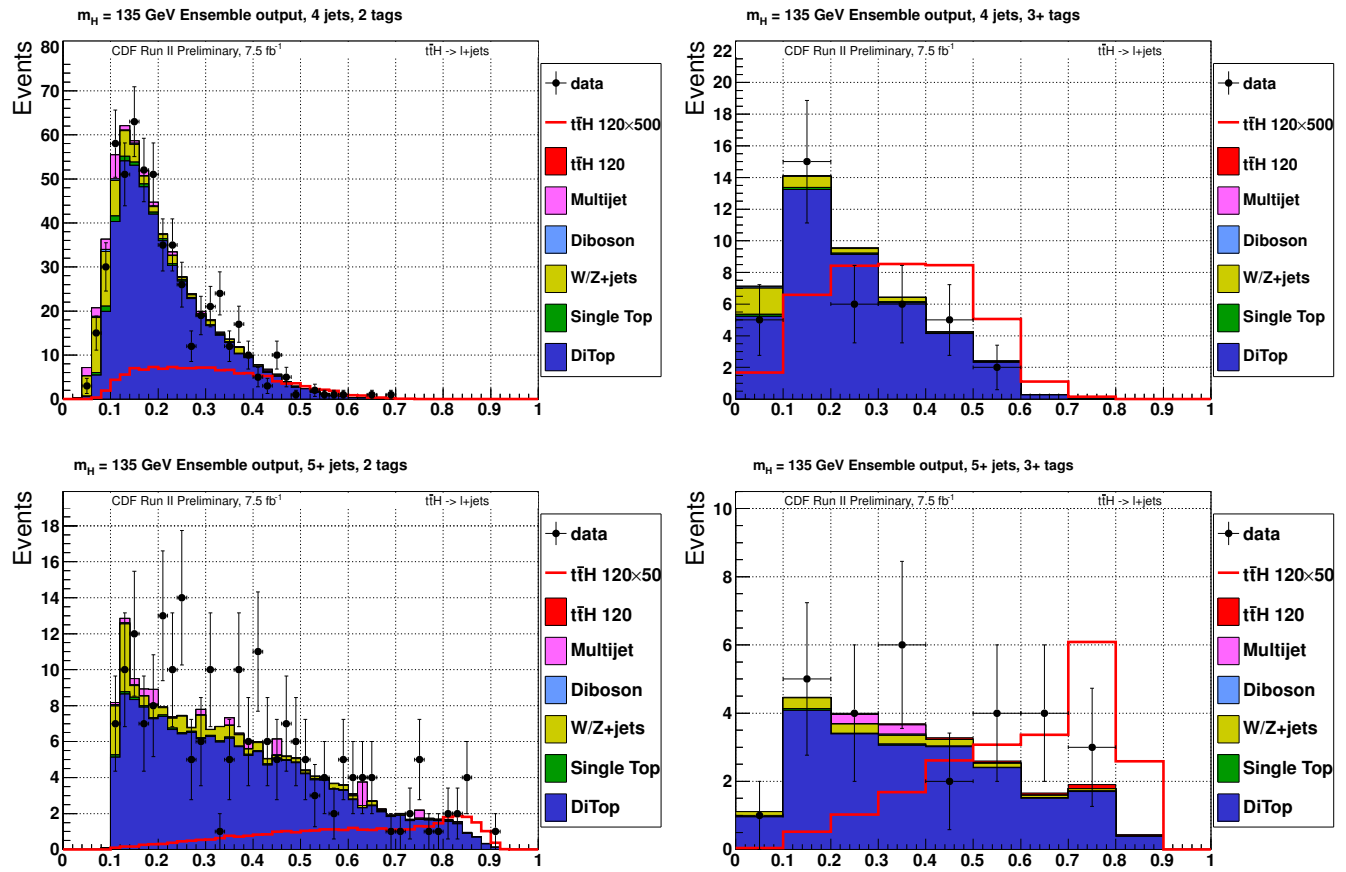

$$
m_{H}=140 \mathrm{GeV} / c^{2}
$$
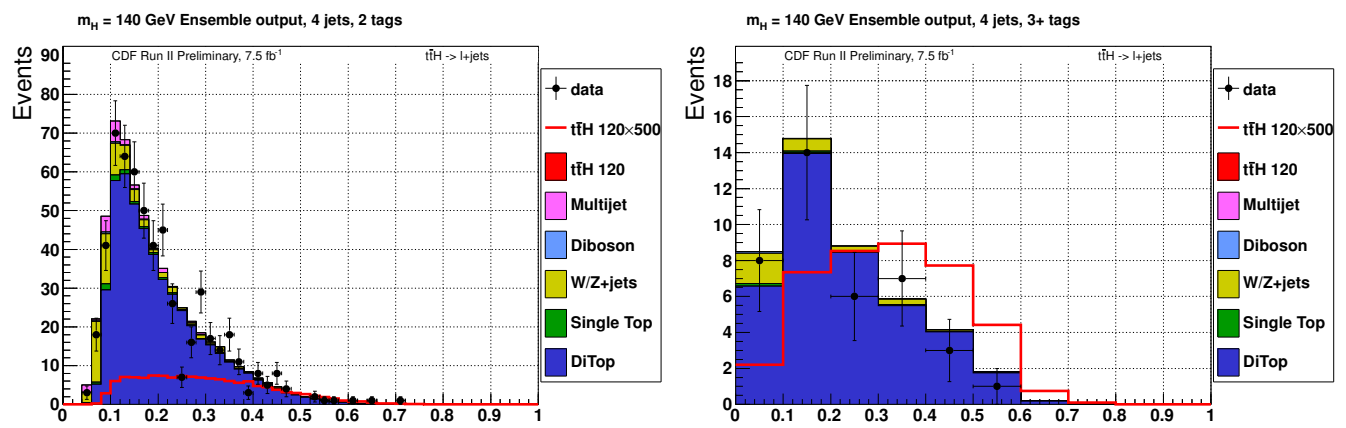

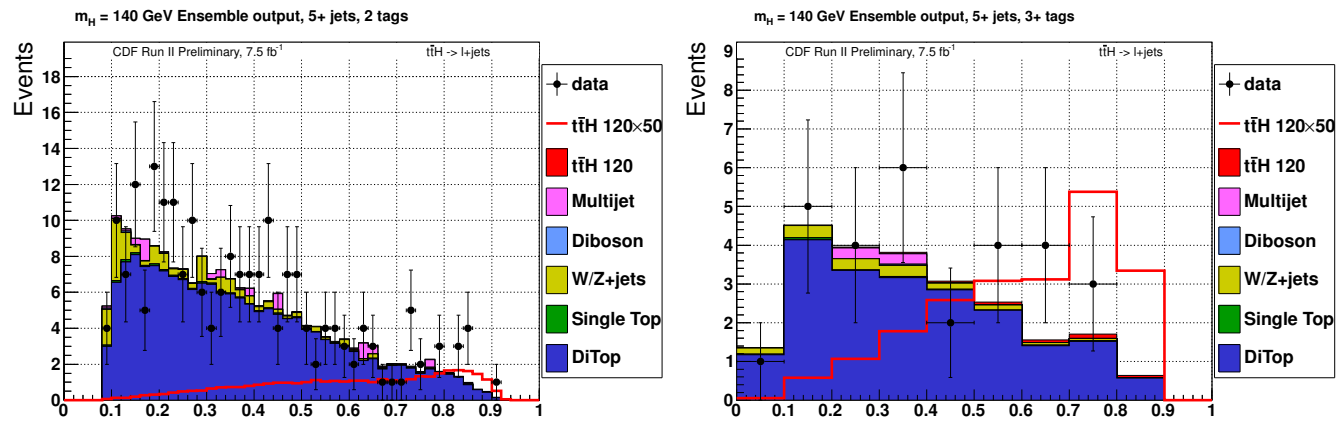

$$
m_{H}=145 \mathrm{GeV} / c^{2}
$$
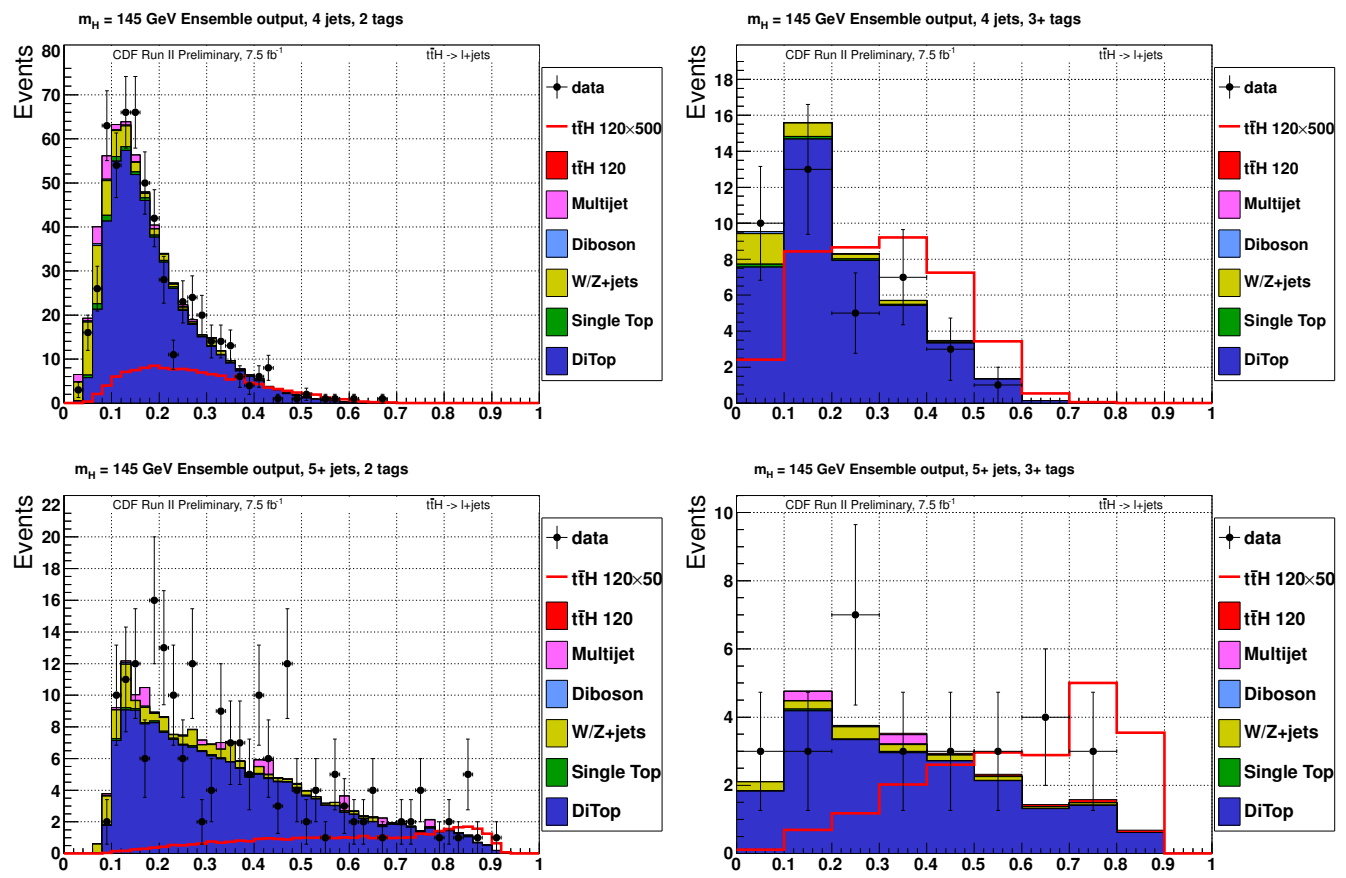

$$
m_{H}=150 \mathrm{GeV} / c^{2}
$$
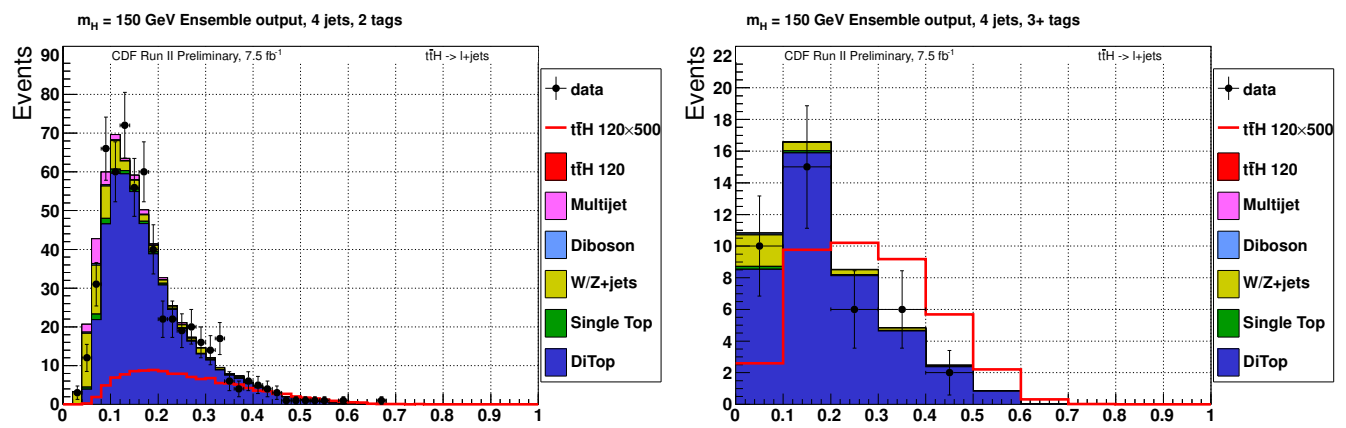

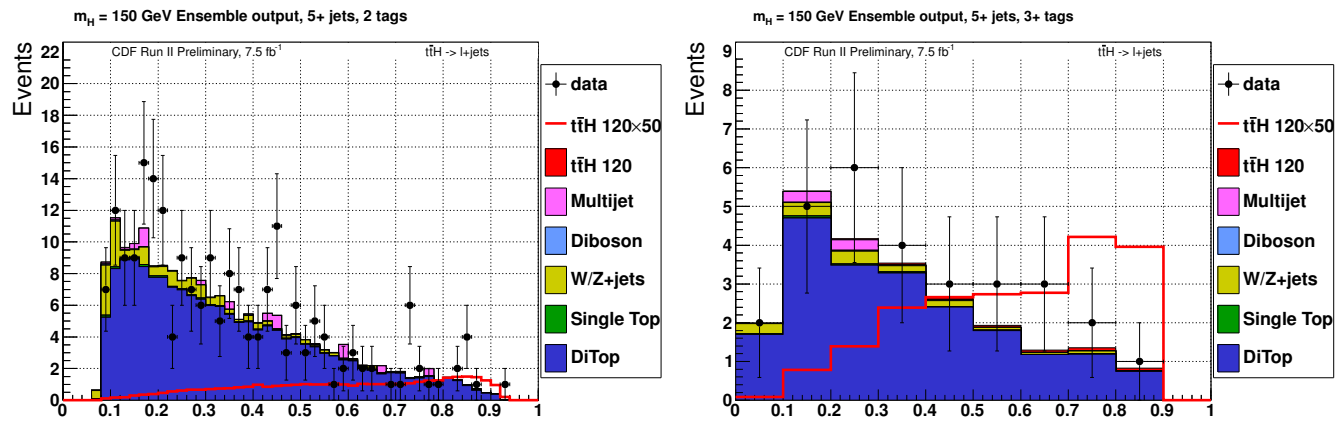

$$
m_{H}=170 \mathrm{GeV} / c^{2}
$$
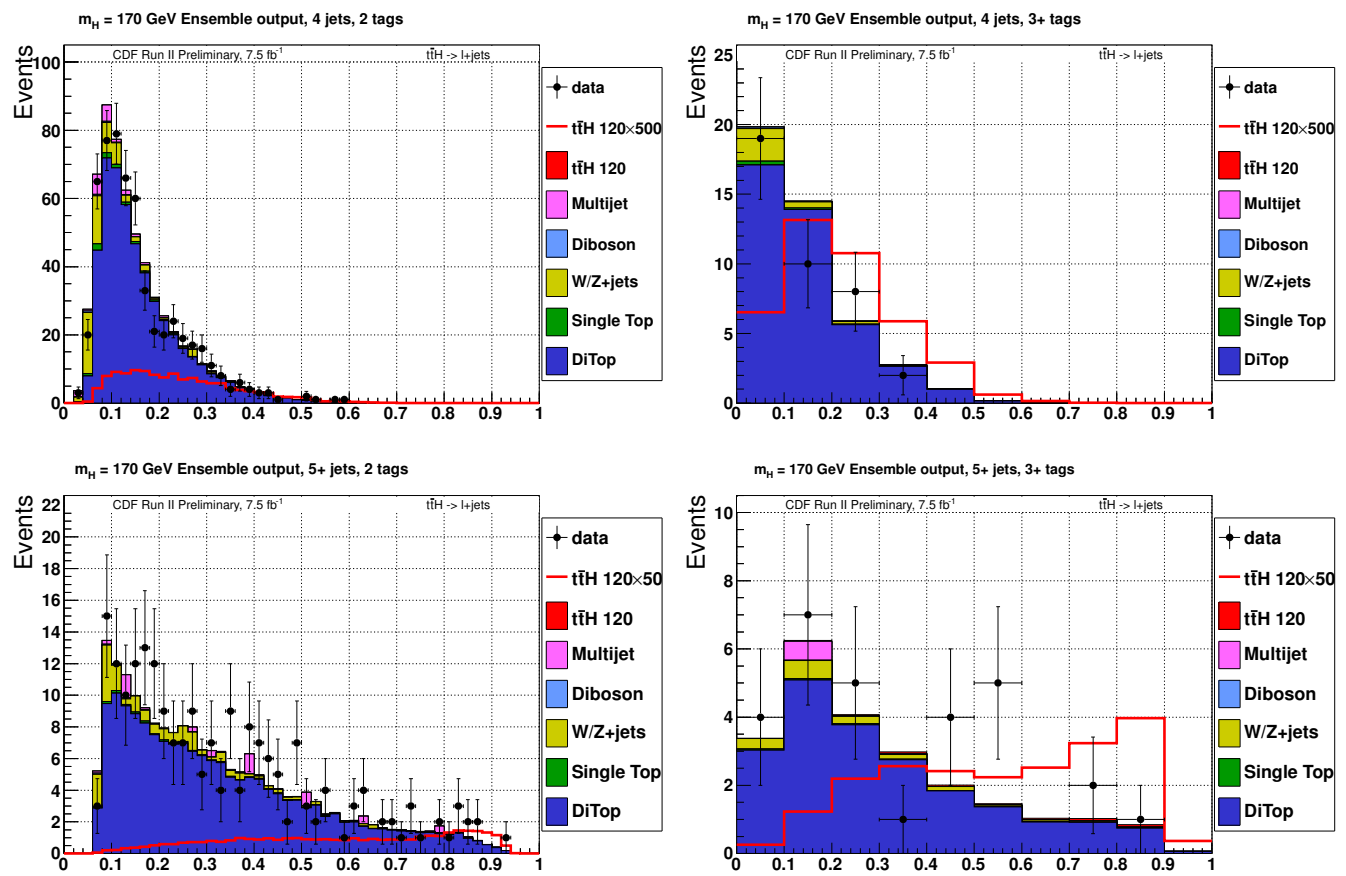